\title{
Molecular characterization of Ptf1a activity during Xenopus embryogenesis
}

\author{
Doctoral Thesis
}

\author{
Dissertation for the award of the degree \\ "Doctor rerum naturalium (Dr. rer. nat.)" \\ in the GGNB program "Genes and Development" \\ at the Georg August University Göttingen \\ Faculty of Biology
}

\author{
submitted by \\ Marie Charlotte Hedderich \\ born in Marburg, Germany
}

Göttingen, August 2012 
Members of the Thesis Committee:

Dr. Kristine A. Henningfeld (Supervisor)

Department of Developmental Biochemistry, University of Göttingen

Prof. Dr. Andreas Wodarz (Reviewer)

Department of Stem Cell Biology, University of Göttingen

Prof. Dr. Anastassia Stoykova

Department of Molecular Cell Biology/Molecular Developmental Neurobiology/Max Planck Institute for Biophysical Chemistry

Prof. Dr. Tomas Pieler (Reviewer)

Department of Developmental Biochemistry, University of Göttingen

Date of the oral examination: 18.10 .2012 


\section{Affidavit}

Herewith, I declare that I prepared the PhD thesis

"Molecular characterization of Ptf1a activity during Xenopus embryogenesis"

on my own and with no other sources and aids than quoted.

Submission date

Göttingen, 31.08.2012

Marie Charlotte Hedderich 


\section{Table of Contents}

Table of Contents.........................................................................................

Acknowledgements .......................................................................... V

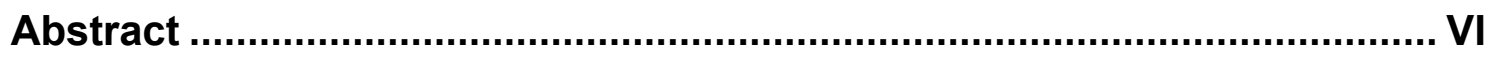

List of Figures .................................................................................. VII

List of Tables ............................................................................................... XI

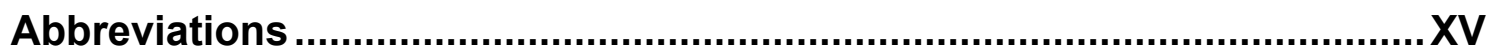

1. Introduction ........................................................................................

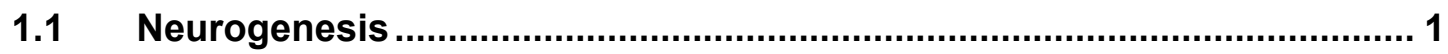

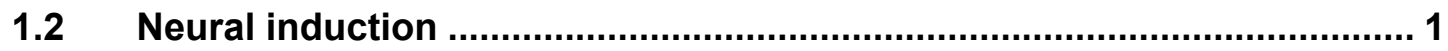

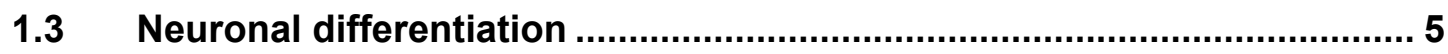

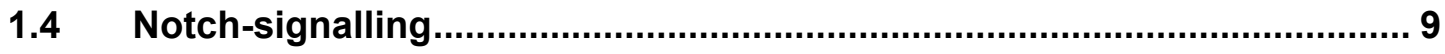

1.5 Neurulation and Neuronal subtype specification ................................ 11

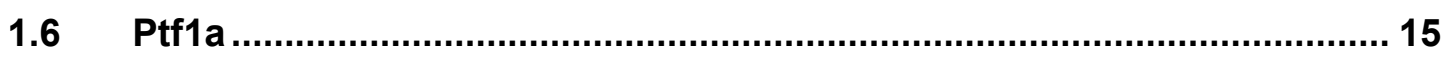

1.6.1 Ptf1a function during development of the pancreas............................. 15

1.6.2 Ptf1a function during development of the nervous system ..................... 17

1.6.3 Ptf1a also forms context-dependent transcription complexes in the nervous

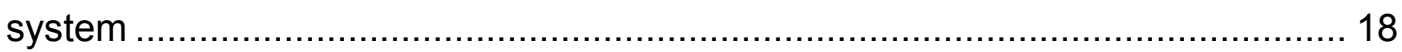

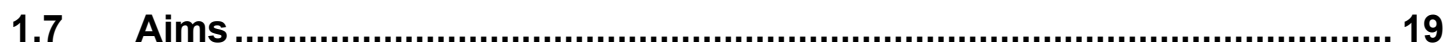

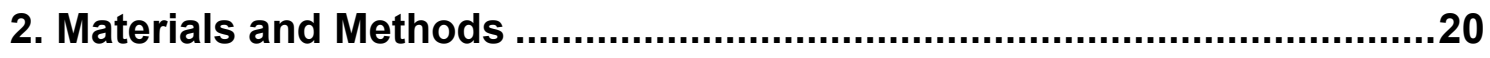

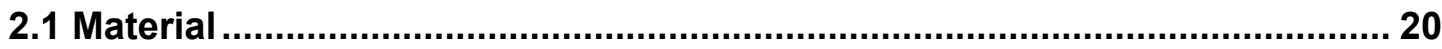

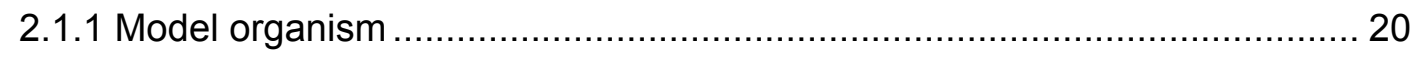

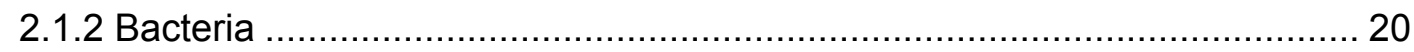

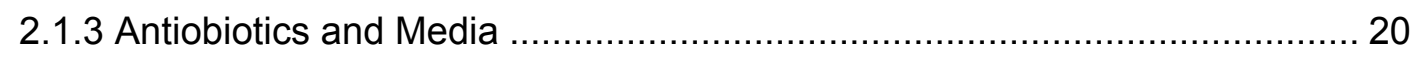

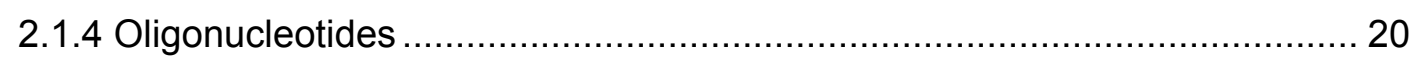

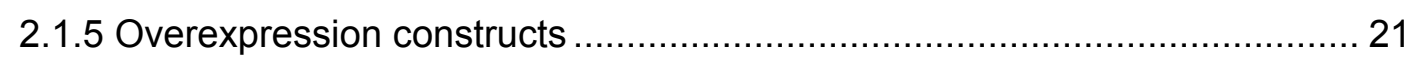

2.1.6 Constructs for real-time RT-PCR standard curves .................................. 23

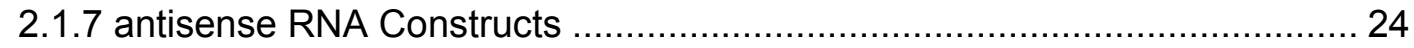

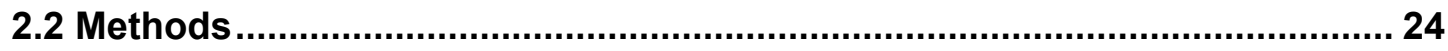

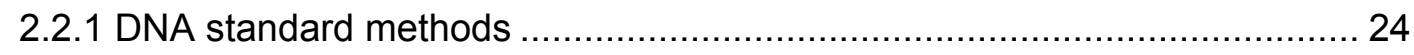

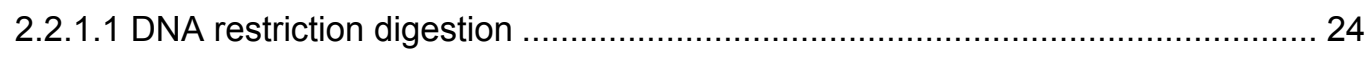

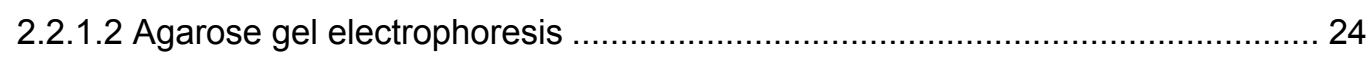

2.2.1.3 Purification of DNA fragments and linearized templates ................................ 24

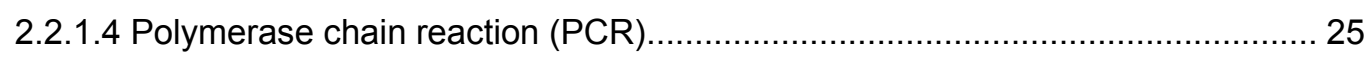




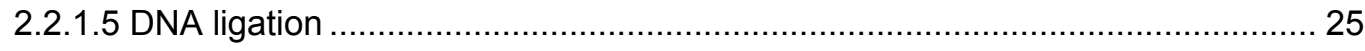

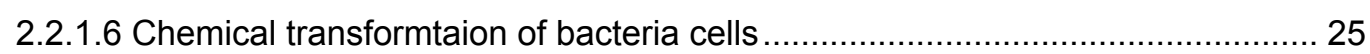

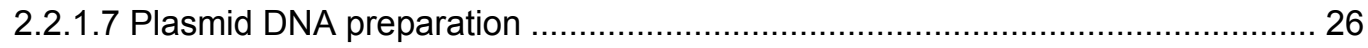

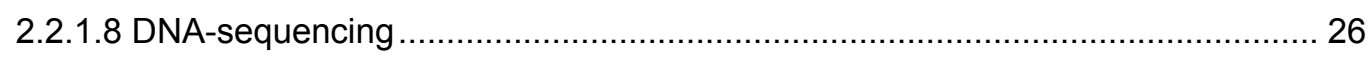

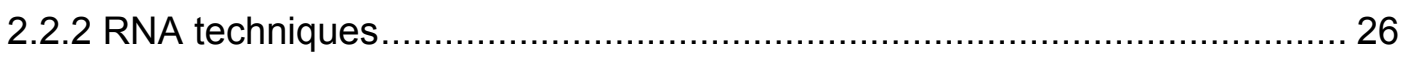

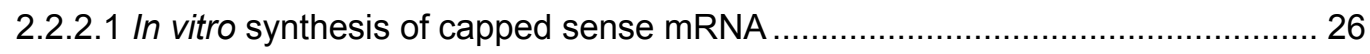

2.2.2.2 In vitro synthesis of antisense RNA ........................................................ 27

2.2.2.3 RNA isolation from ectodermal explants ("animal caps") ............................... 27

2.2.2.3.1 Isolation of total RNA with the "RNAqueous ${ }^{\circledR}-$ Micro" kit (Ambion) ............ 27

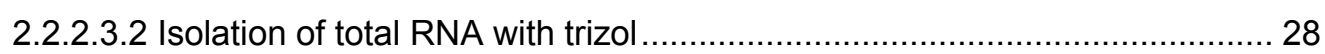

2.2.2.4 Reverse transcription and PCR (semi-quantitative and quantitative RT-PCR) . 28

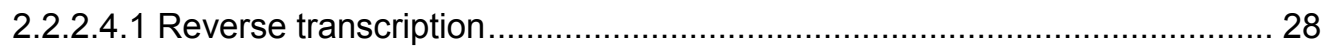

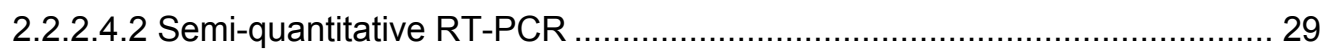

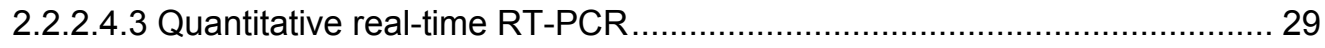

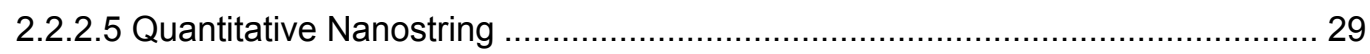

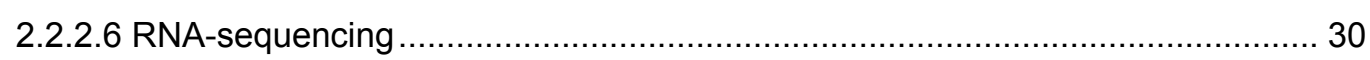

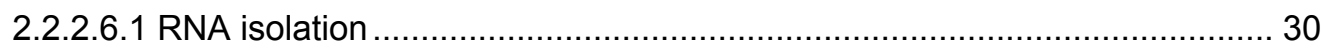

2.2.2.6.2 Sample preparation and sequencing ............................................. 30

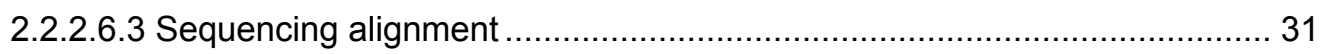

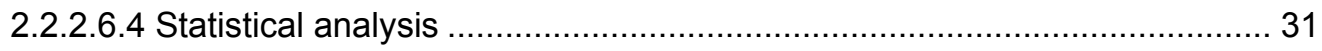

2.2.3 Xenopus laevis embryo culture and microinjections............................... 31

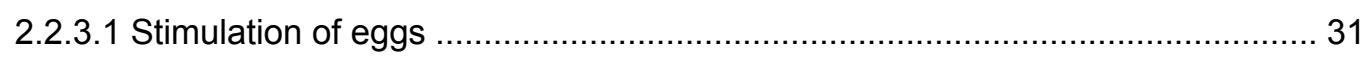

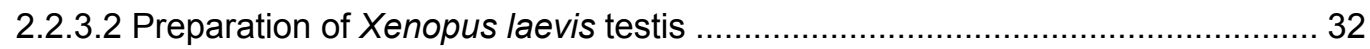

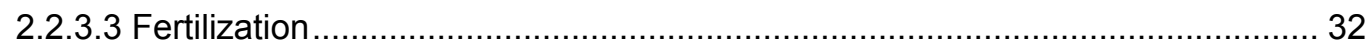

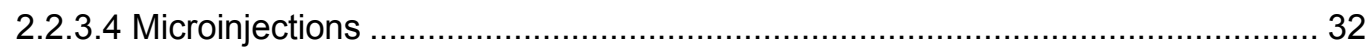

2.2.3.5 Xenopus laevis ectodermal explants ("animal caps") ..................................... 33

2.2.3.6 Treatment of whole embryos and animal caps ............................................. 33

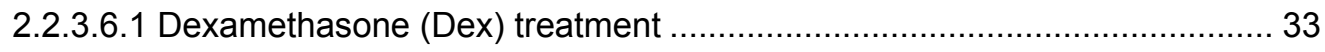

2.2.3.6.2 Cycloheximide (CHX) treatment ................................................... 33

2.2.4 Whole mount in situ hybridization ................................................. 34

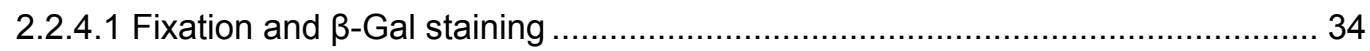

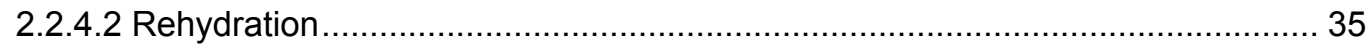

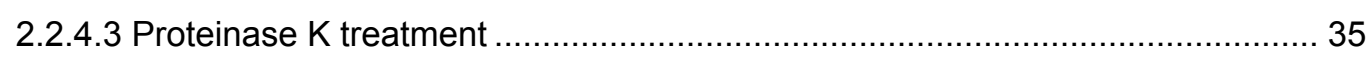

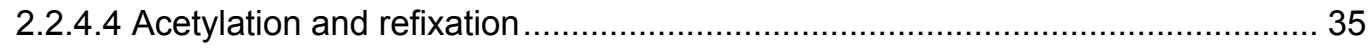

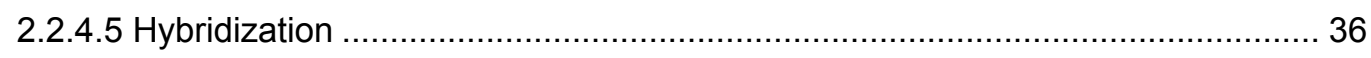

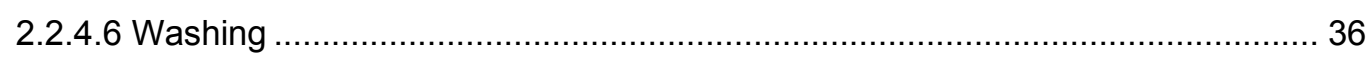

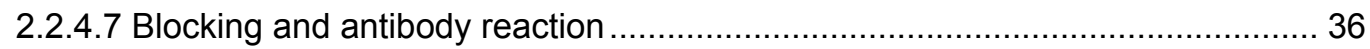

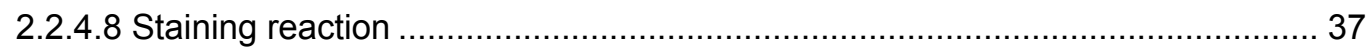

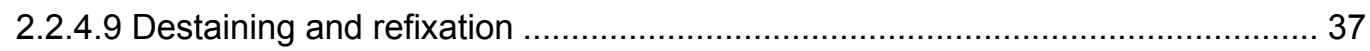

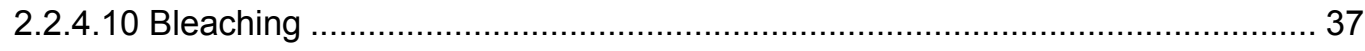

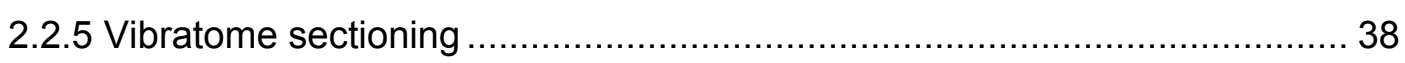




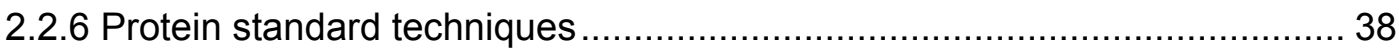

2.2.6.1 Protein-protein interaction in vivo (Co-immunoprecipitation) ........................... 38

2.2.6.2 SDS-Polyacrylamide gel electrophoresis....................................................... 39

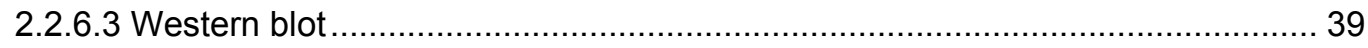

3. Results

3.1 Ptf1a promotes general neurogenesis and specifies GABAergic inhibitory interneurons at the expense of other neuronal cell types.

3.2 Ptf1a and Ngn2 promote general neurogenesis via activation of the same gene network

3.3 Ptf1a is able to drive both general neurogenesis and neuronal subtype specification when induced after neural tube closure. 48

3.4 Ptf1a is maternally expressed in $X$. laevis

3.5 Ptf1a is expressed in the inner ventricular zone of the hindbrain and neural tube 50

3.6 The neuronal subtype-inducing activity of Ptf1a is dominant over Ngn2 ... 52

3.7 The bHLH domain of Ptf1a is not essential for GABAergic interneuronal subtype specification

3.8 Interaction of Ptf1a with $\mathrm{Su}(\mathrm{H})$ is not required for the general neurogenesisinducing activity of Ptf1a 56

3.9 Ptf1a is capable of interacting with $\mathrm{Su}(\mathrm{H})$ at early embryogenesis 59

3.10 Co-expression of Ptf1a and $\mathrm{Su}(\mathrm{H})$ influences expression levels of Ptf1a $\mathrm{Su}(\mathrm{H})$-dependent target genes 60

3.11 Temporal expression analysis of genes induced by Ptf1a, Ptf1a $\mathrm{a}^{\text {W224A/W242A }}$ and Ngn2 by RNA-sequencing

3.12 Analysis of identified downstream targets of Ptf1a, Ngn2 and Ptf1a $a^{\text {W224A/W242A }}$

3.13 The majority of genes induced after 6 and $25 \mathrm{~h}$ by Ptf1a, Ngn2 and Ptf1a $\mathrm{a}^{\mathrm{W} 224 \mathrm{~A} / \mathrm{W} 242 \mathrm{~A}}$ are not shared

3.14 Inhibition of protein synthesis allows earlier activation of Ngn2 expression. 69

3.15 Identification of direct Ptf1a target genes.

3.16 Identification of direct target genes induced in the previous temporal expression analysis by RNA-sequencing . 73

3.17 Analysis of direct targets of Ptf1a and Ptf1a/Su(H). 75

3.18 Identification and validation of direct trimeric-dependent Ptf1a target genes 
4.1 The proneural function of Ptf1a does not require interaction with $\mathrm{Su}(\mathrm{H}) \ldots 81$

4.2 Ptf1 $\mathrm{a}^{\mathrm{W} 224 \mathrm{~A} / \mathrm{W} 242 \mathrm{~A}}$ is a functional transcription factor. 83

4.3 Regulation of gene induction of $\mathrm{Su}(\mathrm{H})$-dependent and independent Ptf1a target genes

4.4 Identification of the genetic program regulated by Ptf1a during $X$. laevis neurogenesis 87

4.5 Identification of novel Su(H)-dependent Ptf1a direct target genes 89

5. Conclusion .91

Bibliography .92

6. Appendix 107

6.1 Summary of processed Nanostring data 107

6.1.1 Experiment 3.2 107

6.1.2 Experiment 3.7 109

6.1.3 Experiment 3.8 and 3.20 111

6.1.4 Experiment 3.10 and 3.21 113

6.2 Summary of the primary Nanostring data. 115

6.2.1 Experiment 3.2 115

6.2.2 Experiment 3.7 117

6.2.3 Experiment 3.8 and 3.20 121

6.2.4 Experiment 3.10 and 3.21 123

6.3 Summary of the genes analysed with the Nanostring 125

6.4 Comparison of genes identified by RNA-sequencing with related publications 127

6.5 Primary transcriptome sequencing data. 129 


\section{Acknowledgements}

First of all, I would like to thank Dr. Kristine Henningfeld for her great supervision, her support and advice, which guided me during this thesis.

Furthermore, I would like to thank Prof. Pieler for his support in many ways, including fruitful discussions and helpful suggestions.

In addition, I would like to thank Prof. Wodarz and Prof. Stoykova for being part of my thesis committee and for providing me with advice and ideas. I also thank Prof. Bucher, Prof. Doenecke and Dr. Prpic-Schäper for being part of my extended thesis committee.

Furthermore, I would like to thank Katja Ditter, Patrick Rolf Berndt, Maja Gere and Juliane Melchert for the nice working atmosphere and for the help during all the experiments. I also would like to thank Lennart Opitz and Dr. Gabriela Salinas-Riester for the extensive help with the RNA-sequencing.

My special thanks go to my family, who always supported and encouraged me throughout my life.

Finally, I would like to especially thank Reinhard Spiekermann for his patience, his support and motivation. 


\section{Abstract}

A balance between inhibitory and excitatory neurons is essential for the establishment of a functional vertebrate nervous system. A key factor in regulating this balance is the bHLH transcription factor Ptf1a, which promotes a GABAergic inhibitory neuronal identity at the expense of a glutamatergic excitatory neuronal identity in the vertebrate retina, hindbrain and spinal cord. In this context, the activity of Ptf1a requires formation of a trimeric complex, in which Ptf1a binds to a commonly expressed bHLH E-protein and to a member of the $\mathrm{Su}(\mathrm{H})$ family. Ptf1a has also been shown to promote general neuronal differentiation in $X$. laevis embryos and explants, suggesting that Ptf1a has proneural activity.

In this thesis work, the role of Ptf1a in the context of both general neurogenesis (early function) and neuronal subtype specification (late function) was investigated. Through the temporal expression analysis of known genes, Ptf1a was shown to drive neurogenesis in animal caps (naïve ectoderm) at early time points through the activation of downstream target genes similar to the proneural transcription factor Ngn2. However, at later stages, Ptf1a activated marker gene expression indicative of GABAergic neurons, while glutamatergic neuronal markers were induced by Ngn2. A mutant version of Ptf1a (Ptf1a ${ }^{\text {W224A/W242A }}$, that is unable to interact with the cofactor $\mathrm{Su}(\mathrm{H})$, maintained the ability to drive general neurogenesis, but was unable to activate GABAergic marker genes. These findings suggest that Ptf1a forms context specific transcription complexes during the development of the nervous system: a $\mathrm{Su}(\mathrm{H})$-independent complex to drive general neurogenesis and $\mathrm{Su}(\mathrm{H})$ dependent complex to specify GABAergic neurons.

As target genes of Ptf1a during the development of the nervous system are not well defined, two independent whole transcriptome analyses were conducted to elucidate the genetic network downstream of Ptf1a. In these assays, a temporal analysis of genes induced by wild-type Ptf1a, Ptf1a W224A/W242A and Ngn2 in $X$. laevis animal caps was performed; direct targets for Ptf1a and Ptf1a/Su(H) were determined by activation of these transcription factors in the presence of an inhibitor of protein synthesis $(\mathrm{CHX})$. Through this approach, many putative 
novel early and late Ptf1a target genes were identified. Further analysis of these downstream targets will give insight into how Ptf1a regulates general neurogenesis and neuronal subtype specification. 


\section{List of Figures}

Figure 1.1 Schematic representation of experiments supporting the 'default model' of neural induction

Figure 1.2 Scheme of the molecular mechanism of neural induction in $X$. laevis

Figure 1.3 X. laevis homologues of the proneural Achaete-scute and Atonal genes of Drosophila

Figure 1.4 Scheme of neuronal differentiation regulated by proneural genes

Figure 1.5 Notch-signalling during development

Figure 1.6 Dorso-ventral patterning of the mouse neural tube

Figure 1.7 Neuronal subtype specification in the $X$. laevis spinal cord

Figure 1.8 Ptf1a forms context-dependent transcription complexes

Figure 3.1 Ptf1a promotes general neurogenesis and specifies GABAergic inhibitory interneurons at the expense of other neuronal cell types

Figure 3.2 Ptf1a and Ngn2 promote general neurogenesis via activation of the same gene network

Figure 3.3 Ptf1a is able to drive general neurogenesis and neuronal subtype specification when induced after neural tube closure 
Figure 3.5 Ptf1a is expressed in the inner ventricular zone of the hindbrain

Figure 3.6 The neuronal subtype-inducing activity of Ptf1a is dominant over Ngn2

Figure 3.7 The bHLH domain of Ptf1a is not essential for GABAergic interneuron subtype specification

Figure 3.8 Interaction of Ptf1a with $\mathrm{Su}(\mathrm{H})$ is not required for the general neurogenesis-inducing activity of Ptf1a

Figure 3.9 Ptf1a is capable of interacting with $\mathrm{Su}(\mathrm{H})$ at early embryogenesis

Figure 3.10 Co-expression of Ptf1a and $\mathrm{Su}(\mathrm{H})$ influences expression levels of Ptf1a Su(H)-dependent target genes

Figure 3.11 Scheme of the alignment process of the sequencing-reads to identify new downstream targets of Ptf1a, Ngn2 and Ptf1a ${ }^{\text {W224A/W242A }}$

Figure 3.12 Temporal expression analysis of Ptf1a-GR, Ngn2-GR and Ptf1a ${ }^{\text {W224A/W242A }}$-GR target genes by whole transcriptome RNA-sequencing

Figure 3.13 Overview of the biological processes enriched in the shared target genes by Ptf1a, Ptf1a ${ }^{\text {W224A/W242A }}$ and Ngn2 after $6 \mathrm{~h}(\mathrm{~A})$ and $25 \mathrm{~h}(\mathrm{~B})$

Figure 3.14 Overview of the biological processes enriched in the $\mathrm{Su}(\mathrm{H})$-dependent Ptf1a target genes after $6 \mathrm{~h}(\mathrm{~A})$ and $25 \mathrm{~h}(\mathrm{~B})$ 
Figure 3.15 Distinct transcripts are present after 6 and $25 \mathrm{~h}$ of Ptf1a-GR, Ngn2-GR and Ptf1a W224A/W242A - GR induction

Figure 3.16 Inhibition of protein synthesis allows earlier activation of Ngn2 expression

Figure 3.17 Scheme of the alignment process of the sequencing reads to identify new direct downstream targets of Ptf1a

Figure 3.18 Identification of direct target genes induced by Ptf1a-GR and $\mathrm{Su}(\mathrm{H})-\mathrm{GR}$ alone and in combination by whole transcriptome RNA-sequencing

Figure 3.19 Enrichment of biological processes in $\mathrm{Ptf1a} / \mathrm{Su}(\mathrm{H})$ direct target genes, which were also present in the time course analysis analysed by RNA-sequencing after $6 \mathrm{~h}(\mathrm{~A})$ and $25 \mathrm{~h}(\mathrm{~B})$

Figure 3.20 Verification of selected direct Ptf1a Su(H)-dependent target genes

Figure 3.21 Co-expression of Ptf1a and $\mathrm{Su}(\mathrm{H})$ influences expression of new identified $\mathrm{Su}(\mathrm{H})$-dependent direct Ptf1a target genes

Figure 4.1 Distinct activities of Ptf1a, its mutated and chimeric version as well as of $\mathrm{Ngn} 2$ in regard to neuronal subtype specification 


\section{List of Tables}

Table 2.1 Summary of Sequencing oligonucleotides and annealing temperature $\left(\mathrm{T}_{\mathrm{A}}\right)$

Table 2.2 Summary of RT-PCR oligonucleotides and working conditions

Table 2.3 Summary of antisense RNA constructs

Table 2.4 Summary of antibodies and working dilutions

Table 3.1 Comparison of the number of genes that were induced

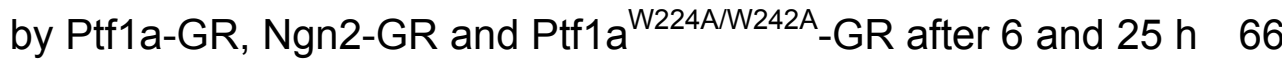

Table 3.2 Identification of direct Ptf1a targets activated after 6 and 25 h 74

Table 3.3 Whole transcriptome RNA-sequencing to identify direct target genes of Ptf1a-GR and Ptf1a-GR together with Su(H)-GR

Table 3.4 Summary of the fold-activation compared to control caps of the selected $\mathrm{Su}(\mathrm{H})$-dependent Ptf1a target genes

Table 6.1 Summary of the averaged fold change over CC of two independent Nanostring experiments for each sample and gene

Table 6.2 Summary of the calculated standard error of the mean (SEM) of the fold change shown in table 6.1 for each sample and gene

Table 6.3 Summary of the averaged fold change over CC of two independent Nanostring experiments for each sample and gene

Table 6.4 Summary of the calculated standard error of the mean (SEM) 110 
of the fold change shown in table 6.3 for each sample and gene

Table 6.5 Summary of the averaged fold change over CC of two independent Nanostring experiments for each sample and gene

Table 6.6 Summary of the calculated standard error of the mean (SEM) of the fold change shown in table 6.5 for each sample and gene

Table 6.7 Summary of the averaged fold change over CC of two independent Nanostring experiments for each sample and gene

Table 6.8 Summary of the calculated standard error of the mean (SEM) of the fold change shown in table 6.7 for each sample and gene

Table 6.9 Primary data of the first Nanostring experiment

Table 6.10 Primary data of the second Nanostring experiment

Table 6.11 Primary data of the first Nanostring experiment

Table 6.12 Primary data of the second Nanostring experiment

Table 6.13 Primary data of the first Nanostring experiment

Table 6.14 Primary data of the second Nanostring experiment

Table 6.15 Primary data of the first Nanostring experiment

Table 6.16 Primary data of the second Nanostring experiment

Table 6.17 Summary of the genes analysed with the Nanostring

Table 6.18 Comparison of direct Ptf1a and Ptf1a/Su(H) target genes with Ptf1a target genes identified in the adult mouse pancreas 
by ChIP-sequencing

Table 6.19 Comparison of direct Ptf1a and Ptf1a/Su(H) target genes with direct Ptf1a target genes identified in the 2-666 pancreatic progenitor cells by ChIP-sequencing

Table 6.20 Comparison of direct Ptf1a target genes with direct $\mathrm{Ngn} 2$ target genes identified in animal caps by Affymetrix Microarray analysis

Table 6.21 Comparison of direct Ptf1a/Su(H) target genes with identified genes downstream of Ptf1a in endodermal explants

Table 6.22 Candidates_Ngn2-GR_6h_Dex_vs_Cc_6h_Dex

Table 6.23 Candidates_Ngn2-GR_25h_Dex_vs_Cc_25h_Dex

Table 6.24 Candidates_Ptf1a-GR_6h_Dex_vs_Cc_6h_Dex

Table 6.25 Candidates_Ptf1a-GR_25h_Dex_vs_Cc_25h_Dex

Table 6.26 Candidates_Ptf1aW224A_W242A-GR_25h_Dex_vs _Cc_25h_Dex

Table 6.27 Candidates_Ptf1aW224A_W242A-GR_6h_Dex_vs_Cc_ 6h_Dex

Table 6.28 VennView_Comparison of downstream targets of Ptf1a, Ngn2 and Ptf1aW224A_W242A after 6 and 25 h

Table 6.29 Candidates_Ptf1a_vs_CC_CHX_Dex 
Table 6.31 Candidates_Ptf1a_SuH_vs_CC_CHX_Dex

Table 6.32 VennView_Comparison of identified direct target genes for Ptf1a and $\mathrm{Su}(\mathrm{H})$ alone as well as in combination

Table 6.33 VennView_Comparison of downstream genes of Ptf1a and Ptf1aW224A_W242A after 6 and $25 \mathrm{~h}$ and genes identified as direct targets of Ptf1a alone and with $\mathrm{Su}(\mathrm{H})$ in combination 


\section{Abbreviations}

\begin{tabular}{|c|c|}
\hline A & Alanin \\
\hline AP & alkaline phosphatase \\
\hline aa & amino acid \\
\hline BCIP & 5-bromo-4-chloro-3-indolyl-phosphate \\
\hline bHLH & basic-helix-loop-helix \\
\hline $\mathrm{BMB}$ & Bohringer Mannheim blocking reagent \\
\hline BMP & bone morphogenetic protein \\
\hline bp & base pairs \\
\hline BSA & bovine serum albumin \\
\hline${ }^{\circ} \mathrm{C}$ & Celsius degree \\
\hline cDNA & complementary DNA \\
\hline $\mathrm{CHX}$ & cycloheximide \\
\hline CIAP & calf intestine alkaline phosphatase \\
\hline Dex & dexamethasone \\
\hline Dig & dioxigenine \\
\hline DNA & desoxyribonucleic acid \\
\hline DNAse & desoxyribonuclease \\
\hline DTT & 1,4-dithiothreitol \\
\hline EDTA & ethylendiaminetetraacetic acid \\
\hline EGTA & $\begin{array}{l}\text { ethylenglycole-bis(2-aminoethylether)-N,N'- } \\
\text { tetraacetate }\end{array}$ \\
\hline et al. & et alii \\
\hline $\mathrm{EtOH}$ & ethanol \\
\hline Gad1 & glutamatic acid decarboxylase \\
\hline h & hour/hours \\
\hline HCG & human chorionic gonadotropin \\
\hline $\mathrm{H}_{2} \mathrm{O}$ & water \\
\hline is & injected side \\
\hline $\mathrm{k}$ & kilo \\
\hline $\mathrm{kb}$ & kilobase \\
\hline I & liter \\
\hline LB & Luria-Bertani \\
\hline
\end{tabular}




\begin{tabular}{|c|c|}
\hline$\mu$ & micro \\
\hline $\mathrm{m}$ & milli \\
\hline M & molar \\
\hline MAB & maleic acid buffer \\
\hline MEM & MOPS-EGTA-MgSO 4 buffer \\
\hline MEMFA & MOPS-EGTA-MgSO ${ }_{4}$ formaldehyde buffer \\
\hline $\min$ & minutes \\
\hline mRNA & messenger RNA \\
\hline$n$ & Nano, number \\
\hline $\mathrm{NaAC}$ & sodium acetate \\
\hline NBT & nitro-blue-tetrazolium \\
\hline Ngn & neurogenin \\
\hline Ngnr & neurogenin-related \\
\hline $\mathrm{nm}$ & nanometer \\
\hline OD & optic density \\
\hline PAGE & polyacrylamid gel electrophoresis \\
\hline PBS & phosphate buffered saline \\
\hline PCR & polymerase chain reaction \\
\hline $\mathrm{pH}$ & negative decade logarithm of hydrogen ion \\
\hline & concentration \\
\hline$\%$ & percentage \\
\hline PTF1 & pancreas transcription factor complex 1 \\
\hline Ptf1a & pancreas transcription factor $1 a$ \\
\hline RNA & ribonucleic acid \\
\hline RNase & ribonuclease \\
\hline rpm & rounds per minute \\
\hline RT & room temperature, reverse transcriptase \\
\hline RT-PCR & reverse transcriptase PCR \\
\hline SDS & sodium dodecyl sulfate \\
\hline $\mathrm{sec}$ & second \\
\hline $\mathrm{SHH}$ & Sonic Hedgehog \\
\hline SSC & standard saline citrate buffer \\
\hline st & stage \\
\hline $\mathrm{T}_{\mathrm{A}}$ & annealing temperature \\
\hline
\end{tabular}




$\begin{array}{ll}\text { Taq } & \text { Thermus aquaticus } \\ \mathrm{T}_{\mathrm{m}} & \text { melting temperature } \\ \mathrm{U} & \text { units } \\ \mathrm{V} & \text { voltage } \\ \text { Vol. } & \text { volume } \\ \text { W } & \text { Tryptophan } \\ \text { wmish } & \text { whole mount in situ hybridization } \\ \text { X-Gal } & \text { 5-bromo-4-chloro-3-indolyl- } \beta \text { - } d \text {-galactoside }\end{array}$




\section{Introduction}

\subsection{Neurogenesis}

A primary aim in the field of neurobiology is the development of therapeutic treatments for neurodegenerative and other neurological diseases. Many promising strategies are based on the manipulation of cells in vitro and in vivo. It is therefore essential to understand the molecular mechanisms by which a progenitor cell acquires a neuronal fate and subsequently obtains a specific neuronal subtype identity.

The African clawed frog Xenopus laevis ( $X$. laevis) is an ideal vertebrate model organism to study the development of the vertebrate nervous system. The first primary neurons are born in the open neural plate in a relative simplistic pattern (Hartenstein, 1989; Chitnis et al., 1995). Moreover, owing to the external development, the earliest stages of embryogenesis are easily accessible for manipulations, such as microinjection of DNA, mRNA or antisense morpholino oligonucleotides. In addition, explant and transplantation experiments are easy to perform. The animal caps are pluripotent embryonic cells derived from the ectoderm of blastula stage embryos and are an attractive system to study in vitro differentiation (Borchers and Pieler, 2010). As the $X$. laevis genome is partially tetraploid, the closely related species Xenopus tropicalis (X. tropicalis) is increasingly being used as it has a sequenced diploid genome (Amaya, 2005; Hellsten et al., 2010; Harland and Grainger, 2011). However, routine use of $X$. tropicalis as a model system is hindered by the faster development of early cleavage stages and the more difficult cultivation of the embryos.

\subsection{Neural induction}

The development of the nervous system begins with the process of neural induction, during which ectodermal cells become committed to a neural cell fate (Rogers et al., 2009b). X. laevis explant experiments demonstrated that the neural fate of ectodermal cells is specified during gastrulation. While ectodermal explants (animal caps) of blastula stage embryos develop into epidermal tissue, those from gastrula stage embryos give rise to neural tissue 
(Hamburger, 1969; Sanes, Reh and Harris, 2006). However, upon dissociation of $X$. laevis blastula ectodermal explants, cells obtain a neural instead of an epidermal fate, implicating that in the absence of cell-to-cell signalling a neural fate is specified (Godsave and Slack, 1989; Grunz and Tacke, 1989; Sato and Sargent, 1989; Wilson and Hemmati-Brivanlou, 1995) (Fig. 1.1). This is further supported by the rescue of an epidermal cell fate in dissociated cells when cultivated in the presence of BMP-4 (Wilson and Hemmati-Brivanlou, 1995) (Fig. 1.1). Similarly, upon overexpression of a dominant-negative activin receptor, animal caps also acquire a neural fate, further indicating that inhibition of the TGF-beta signalling-pathway is required for neural cell fate determination (Hemmati-Brivanlou and Melton, 1994) (Fig. 1.1). These initial experiments led to the so called 'default model', suggesting that the neural identity of dorsal ectodermal cells is the 'default' state and that BMP-signalling must actively be inhibited.

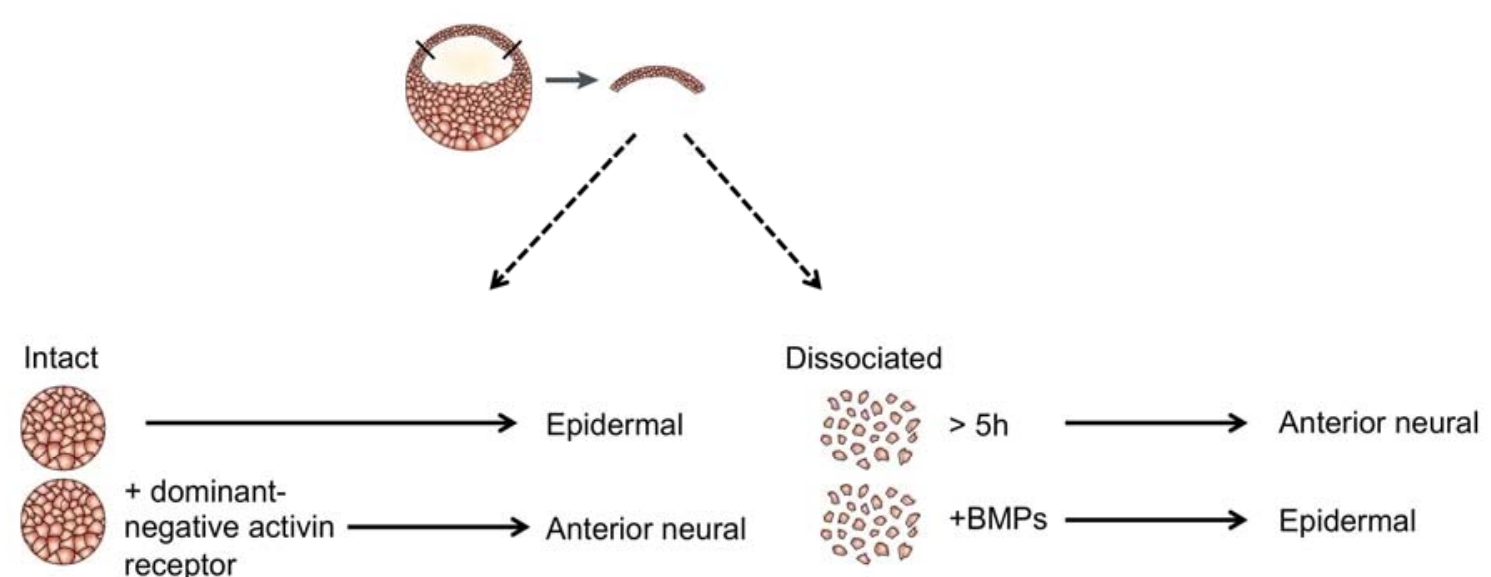

Figure 1.1 Schematic representation of experiments supporting the 'default model' of neural induction.

Ectodermal explants (animal caps) excised from the animal pole of $X$. laevis blastula stage embryos, develop into atypical epidermis. However, upon overexpression of a dominant-negative activin receptor or dissociation of the animal caps, the cells acquire a neural cell fate. An epidermal cell fate can be restored, if dissociated animal caps are cultivated in the presence of BMPs. (Muñoz-Sanjuán and Brivanlou, 2002, modified)

Several BMP-antagonists including Chordin (Piccolo et al., 1996; Sasai et al., 1994), Noggin (Zimmerman et al., 1996; Lamb et al., 1993) and Cerberus (Bouwmeester et al., 1996; Piccolo et al., 1999) have been identified. These factors all have neural inducing activity and are expressed in the Spemann Organizer, which during gastrulation involutes and underlies the prospective neuroectoderm. Moreover, they are all secreted factors that inhibit BMPsignalling by binding BMP-molecules in the extracellular space and prevent 
receptor activation (Fig. 1.2). BMP activity is further attenuated by FGFsignalling (Furthauer et al., 1997; Wilson et al., 2000), which inactivates Smad1 (Fig. 1.2), an intracellular effector of the BMP-pathway (Pera et al., 2003).

The 'default model' of neural induction remains controversial as several experimental findings, particularly in higher vertebrates, are inconsistent with this hypothesis. In this regard, individual knock-out mice of the neural inducer molecules Noggin, Chordin or Follistatin show no defect in neural induction (Bachiller et al., 2000; Bachiller et al., 2003; Matzuk et al., 1995; McMahon et al., 1998). However, functional redundancy of the multiple neural inducer molecules cannot be excluded. Furthermore in chick, the BMP-inhibitors are not sufficient to induce neural cells in epidermal or extra-embryonic ectodermal tissue and their temporal expression pattern does not coincide with the ability of the chick organizer to induce neural cells (Wilson and Edlund, 2001; Wilson et al., 2000; Streit et al., 1998; Storey et al., 1992; Levin, 1998). In summary, these data indicate that an inhibition of BMP-signalling alone is not sufficient to induce neural fate.

Several experiments implicate a role for FGF-signalling in neural induction, independently from its function to attenuate BMP-signalling. In $X$. laevis, experiments demonstrated that the induction of neural cell fate in nonneural ectoderm requires both, inhibition of BMP-signalling and active FGFsignalling (Wawersik et al., 2005; Delaune et al., 2005; Linker and Stern, 2004). This notion is further supported by the fact that in $X$. laevis and chick, blockage of FGF-signalling disturbs neural development (Streit et al., 2000; Hongo et al., 1999) and that overexpression of a dominant-negative FGF-receptor renders Noggin and Chordin unable to induce neural cell fate (Launay et al., 1996; Sasai et al., 1996). FGF alone is also able to induce ectopic expression of neural markers in $X$. laevis ectodermal cells as well as in chick epiblast cells (Lamb and Harland, 1995; Kengaku and Okamoto, 1995; Storey et al., 1998; Alvarez et al., 1998).

In addition to FGF-signalling and inhibition of the BMP-pathway, $\mathrm{Ca}^{2+}$ signalling has been implicated in neural induction (Leclerc et al., 2012). Spontaneous elevations of intracellular $\mathrm{Ca}^{2+}$ occur in dorsal ectodermal cells at blastula stage and $\mathrm{Ca}^{2+}$-levels increase during gastrulation (Leclerc et al., 2000; Leclerc et al., 1997). Furthermore, blocking $\mathrm{Ca}^{2+}$-signalling abolishes neural 
induction (Leclerc et al., 2012), whereas an increase in $\mathrm{Ca}^{2+}$ is sufficient to induce neural cells (Moreau et al., 1994) .

Several transcription factors are expressed in the neuroectoderm during neural induction and are involved in neural fate stabilization (Rogers et al., 2009b) (Fig. 1.2). One important group is the Zic family of zinc-finger transcription factors. Both Zic1 and Zic3 are activated in the ectoderm through inhibition of the BMP-pathway (Nakata et al., 1997; Mizuseki et al., 1998a; Marchal et al., 2009) and Zic3 is additionally a target of $\mathrm{Ca}^{2+}$-signalling (Leclerc et al., 2003). Overexpression of both members of the Zic family, as well as any of the three Iroquois genes, Iro1, Iro2 and Iro3, not only expands the neural plate, but also promotes the onset of neural differentiation factors (Bellefroid et al., 1998; Gomez-Skarmeta et al., 1998). Overexpression of Zic2, another member of the Zic family, also expands the neural plate, however, it represses the expression of neural differentiation genes (Brewster et al., 1998). This suggests that Zic2 expression maintains neural cells in an undifferentiated cell state. Additionally, three members of the Sry-related HMG-box family, Sox2, Sox3 and SoxD are involved in neural fate stabilization. Sox 2 and Sox3 are thought to be important for neural progenitor maintenance (Hardcastle and Papalopulu, 2000; Ellis et al., 2004; Pevny and Placzek, 2005; Rogers et al., 2009a), whereas SoxD was shown to activate the expression of the neural determination gene Neurogenin 2 (Ngn2) and to promote delayed neural differentiation (Mizuseki et al., 1998b). Further pan-neural genes involved in progenitor maintenance are the forkhead transcription factor FoxD5 and the coiled-coil protein Geminin (Seo and Kroll, 2006; Kroll, 2007; Kroll et al., 1998; Pitulescu et al., 2005; Sullivan et al., 2001). Several studies indicate a cross-talk between these genes, which thereby regulates the balance between neural fate stabilization and neural differentiation (Rogers et al., 2009b). 


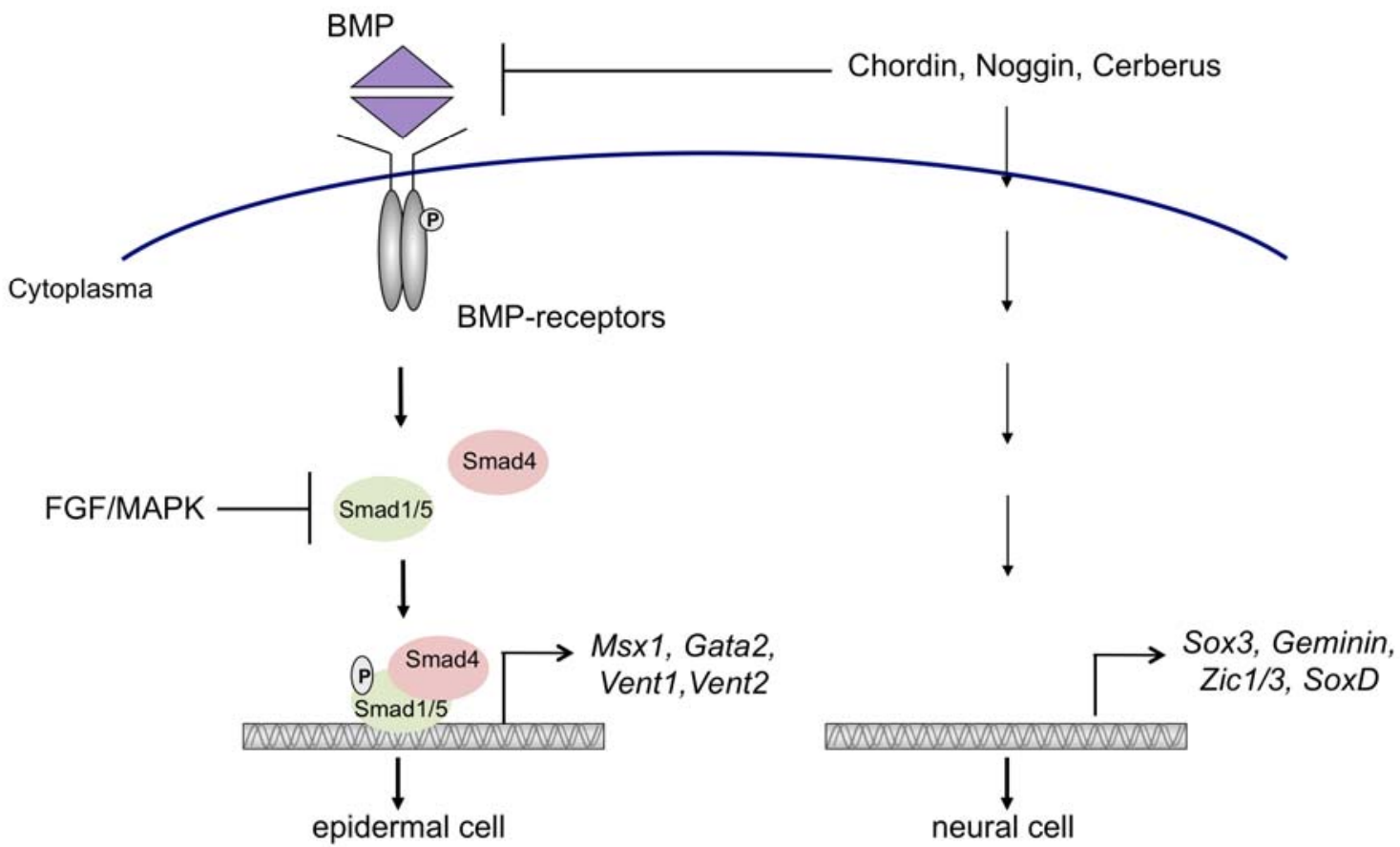

Figure 1.2 Scheme of the molecular mechanism of neural induction in $X$. laevis. Upon BMP binding to the receptors, the BMP-receptors dimerize and undergo autophosphorylation. Through the activation of target genes such as Gata2, Ms 1 , Vent1 and Vent2, BMP-signalling induces an epidermal cell fate. However, BMPsignalling can be intracellular inhibited through the FGF/MAPK pathway, as well as extracellular through the secreted molecules Noggin, Cerberus and Chordin. Through the inhibition of BMP-signalling, multiple genes are activated, including Zic1, Zic3 and SoxD, which lead to neural differentiation of ectodermal cells. Progenitor pools are maintained by the induction of genes like Sox3 and Geminin. (after Rogers et al., 2009b)

\subsection{Neuronal differentiation}

In vertebrates, the progenitor cells within the neural plate are highly mitotic (Hartenstein, 1989) and become differentiated due to the expression of proneural genes. The first proneural basic-helix-loop-helix (bHLH) genes discovered were the genes of the Achaete-scute complex in Drosophila (Murre et al., 1989). These genes, as well as the gene Atonal (Jarman et al., 1993), induce neural commitment of ectodermal cells and were therefore termed proneural genes (Ghysen and Dambly-Chaudiere, 1989; Jan and Jan, 1994). Several vertebrate homologues have been identified, including in $X$. laevis, and can be classified into Atonal and Achaete-scute related genes (Fig. 1.3) (Lee, 1997; Guillemot, 1999). Through the conserved bHLH domain, these factors form dimers, either homo- or heterodimeric complexes with an ubiquitously expressed class A bHLH transcription factor. These complexes bind a 
characteristic sequence known as the E-box motif (CANNTG) within regulatory elements of target genes (Murre et al., 1989).

In vertebrates, proneural genes induce neuronal cell fates of neural cells and are key regulators of the neurogenic differentiation program. However, not all members of the Achaete-scute and Atonal gene family have an endogenous proneural function, as NeuroD1, NeuroD6/Ath2 and NeuroD4/Ath3 are only expressed in post-mitotic neurons.

\begin{tabular}{|c|c|c|c|c|}
\hline Family & Gene & Onset of expression & Expression st. 14 & Reference \\
\hline \multirow[t]{2}{*}{ Achaete-scute } & Ascl1Xash1 & st. 21 & - & Ferreiro et al., 1993 \\
\hline & Asc/3/Xash3 & st.11.5 & $\begin{array}{l}\text { one stripe encompassed by the medial and } \\
\text { intermediate stripe of primary neurons, which } \\
\text { ends in an anterior transversal stripe }\end{array}$ & Turner et al., 1994, Bellefroid et al., 1998 \\
\hline \multirow[t]{8}{*}{ Atonal } & Ngn1 & st.11 & three stripes of primary neurons, tp & Nieber et al., 2009 \\
\hline & Ngn2/Ngnr1 & st.10.5/11 & three stripes of primary neurons, tp & Ma et al., 1996, Nieber et al., 2009 \\
\hline & Ngn3 & st.11 & $\begin{array}{l}\text { one stripe between medial and intermediate } \\
\text { stripe of primary neurons }\end{array}$ & Nieber et al., 2009 \\
\hline & Atoh1/Xath1 & st.17 & - & Kim et al., 1997 \\
\hline & NeuroD6/Xath2 & st.32 & - & Taelman et al., 2001 \\
\hline & Atoh7Xath5 & st. 17 & - & Kanekar et al., 1997 \\
\hline & NeuroD4/Xath3 & st.12 & three stripes of primary neurons, tp & Perron et al., 1999 \\
\hline & NeuroD1 & st. 14 & $\begin{array}{l}\text { two stripes representing medial and lateral } \\
\text { stripe of primary neurons, tp }\end{array}$ & Lee et al., 1995 \\
\hline
\end{tabular}

Figure 1.3 X. laevis homologues of the proneural Achaete-scute and Atonal genes of Drosophila.

Due to conserved sequences in the bHLH domain, $X$. laevis proneural bHLH transcription factors can be classified into the Achaete-scute and the Atonal gene family. The table summarizes the onset of gene expression as well as the expression domains of the identified proneural bHLH transcription factors on each side of the midline in $X$. laevis at st. 14 (open neural plate). tp, trigeminal placode

The vertebrate proneural genes include the members of Neurogenin family (Sommer et al., 1996; Nieber et al., 2009), Ascl1/Ash1 (Ferreiro et al., 1993; Talikka et al., 2002) and Asc/3/Ash3 (Turner and Weintraub, 1994; Chitnis and Kintner, 1996) as well as Atoh1/Ath1 (Kim et al., 1997) and Atoh7/Ath5 (Kanekar et al., 1997). During neuronal differentiation, the proneural genes have multiple functions, which induce the process of neuronal differentiation and maturation (Fig. 1.4 A) (Bertrand et al., 2002; Guillemot, 1999; Castro and Guillemot, 2011). To promote neuronal commitment of a cell, 
proneural genes activate a cascade of neuronal differentiation factors (Guillemot, 1999; Bertrand, 2002), regulate cell migration (Ge et al., 2006) and cell-cycle withdrawal (Castro et al., 2011; Klisch et al., 2011) (Fig. 1.4 A). In addition, the Notch-signalling pathway is also activated by the proneural bHLH factors, which regulates the balance between neural progenitors and neuronal differentiated cells (see chapter 1.4).

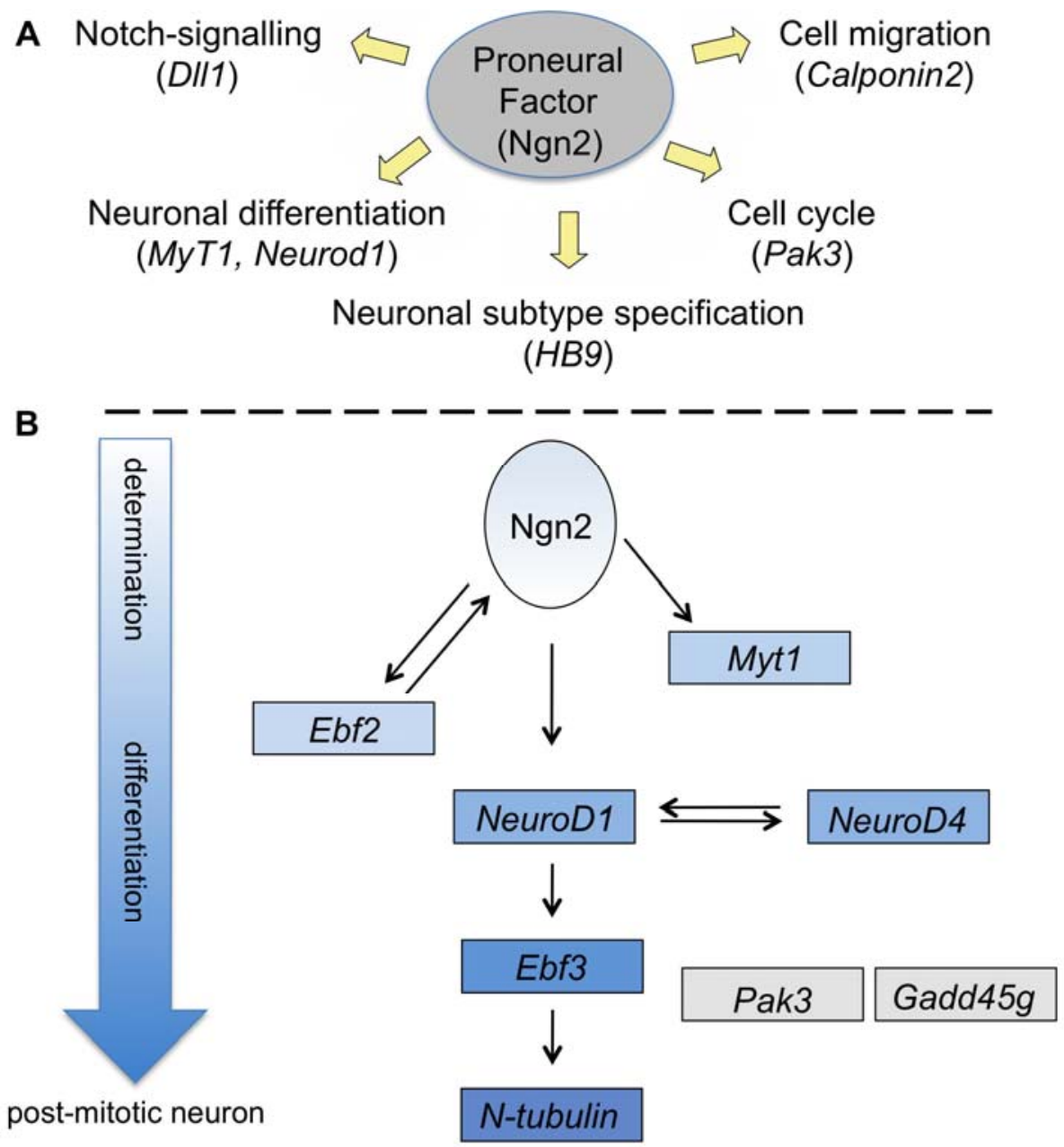

Figure 1.4 Scheme of neuronal differentiation regulated by proneural genes.

(A) Vertebrate proneural genes such as Ngn2 confer distinct activities to induce neuronal commitment of a cell, including activation of Notch-signalling, promotion of cell migration and neuronal differentiation as well as regulation of the cell cylce. Furthermore, proneural genes are also involved in neuronal subtype specification. (B) Due to the onset of proneural gene expression as e.g. Ngn2, a neural cell obtains neuronal cell identity. Ngn2 is known to induce a cascade of differentiation factors in $X$. laevis, such as Myt1, Ebf2, NeuroD4, Ebf3 and NeuroD1, which lead to the induction of $N$-tubulin. In addition, Ngn2 activates the cell cycle inhibitors Pak3 and Gadd45g.

In $X$. laevis, the primary nervous system is essential for the movements and responses of the larvae (Roberts, 2000). Prior to morphogenesis, a second round of neurogenesis starts in $X$. laevis and replaces the majority of primary 
neurons. Several studies indicate that many factors involved in primary neurogenesis are also expressed during secondary neurogenesis, suggesting that the molecular mechanism between the two processes is conserved (Schlosser et al., 2002; Wullimann et al., 2005).

In $X$. laevis, the first primary neurons differentiate at the open neural plate stage in three longitudinal stripes on each side of the midline, as well as in the trigeminal ganglia (Fig. 1.7 A) (Chitnis et al., 1995). Post-mitotic neurons can be visualized by the expression of class II beta-tubulin ( $N$-tubulin) (Oschwald et al., 1991). Neuronal differentiation in the anterior neural plate, with exception of the trigeminal ganglia, is delayed until tadpole stages (Papalopulu and Kintner, 1996).

The best-characterised proneural gene during $X$. laevis primary neurogenesis is the Atonal ortholog and neuronal determination factor Neurogenin 2 (Ngn2, also known as Ngnr-1) (Ma et al., 1996). Ngn2 is expressed already during early gastrula stage (stage 10.5/11) in the prospective neural ectoderm (Ma et al., 1996; Nieber et al., 2009). In the open neural plate, it prefigures the characteristic three longitudinal stripes of primary neurons on each side of the midline (Bellefroid et al., 1996). Ngn2 confers all the abovedescribed activities to be classified as a proneural bHLH transcription factor (Fig. 1.4 A). It induces a cascade of differentiation factors (Fig. 1.4 B.), including the bHLH proteins NeuroD1 (Ma et al., 1996), NeuroD4 (Ath3) (Perron et al., 1999), the zinc finger HLH transcription factors Ebf2 (Dubois et al., 1998) and Ebf3 (Pozzoli et al., 2001) as well as the proneural RNA binding protein Seb4R (Boy et al., 2004). In addition, Ngn2 activates the zinc finger transcription factor Myt1, which allows the cells fated to become a neuron to escape lateral inhibition (Bellefroid et al., 1996). All these genes downstream of Ngn2 are also expressed in the characteristic stripe-like pattern at the open neural plate and form a synexpression group (Niehrs and Pollet, 1999). Furthermore, genes that regulate cytoskeleton or migratory events in the context of the nervous system development are targets of Ngn2 (Seo et al., 2007) as well as genes that promote cell-cycle withdrawal such as the growth arrest and DNA-damageinducible gamma (Gadd45g) gene and Pak3 (Souopgui et al., 2002; de la CalleMustienes et al., 2002) (Fig. 1.4 B). Additional proneural bHLH transcription factors are also expressed in the open neural plate, but show expression in 
divergent domains (Fig. 1.3). Following neural tube closure, the individual proneural bHLH transcription factors are expressed in discrete domains, suggesting that they are essential for generation of post-mitotic neurons and neuronal subtype identities in distinct domains of the CNS. Indeed several proneural factors such as Ngn2 (Perron et al., 1999) and Ath5 (Kanekar et al., 1997) have been also shown to play a role in neuronal subtype specification.

\subsection{Notch-signalling}

The Notch-signalling pathway regulates the balance between progenitor maintenance and differentiation (Lewis, 1996). During $X$. laevis primary neurogenesis, the process of lateral inhibition is mediated by the Notchsignalling and generates a salt and pepper like pattern of the neurons at the open neural plate stage (Fig. 1.5 A). The Notch pathway acts through cell-to-cell signalling with cells being defined as either signal-sending or signal-receiving. Proneural genes such as Ngn2 and Ascl1 activate the expression of the transmembrane Notch-ligands in the signal-sending cell (Fig. 1.4 B) (Chitnis et al., 1995; Chitnis and Kintner, 1996; Ma et al., 1996; Henke et al., 2009a). In vertebrates, several such ligands are known (Bray, 2006), including DII1, 2, and 4 as well as Jag1 and 2 in $X$. laevis. The ligands bind one of the four transmembrane Notch-receptors (Bray, 2006) on the neighbouring cell, which induces a series of proteolytic cleavages in the Notch-receptor. As a result, the intracellular domain of the Notch-receptor (Notch-ICD) is released and translocates to the nucleus (Schroeter et al., 1998; Chan and Jan, 1999; Selkoe and Kopan, 2003). In the nucleus, Notch-ICD binds a DNA-binding protein of the CSL family $(\mathrm{Su}(\mathrm{H}))$ in $X$. laevis and Drosophila, Rbp-j in mouse, Lag-1 in C. elegans) (Honjo, 1996), which acts in a complex as a transcriptional repressor. However, after binding of Notch-ICD, the repressor is converted into an activator and together with co-factors activates the transcription of the Notchtarget genes (Wettstein et al., 1997). 
A

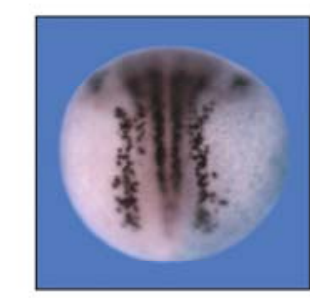

B

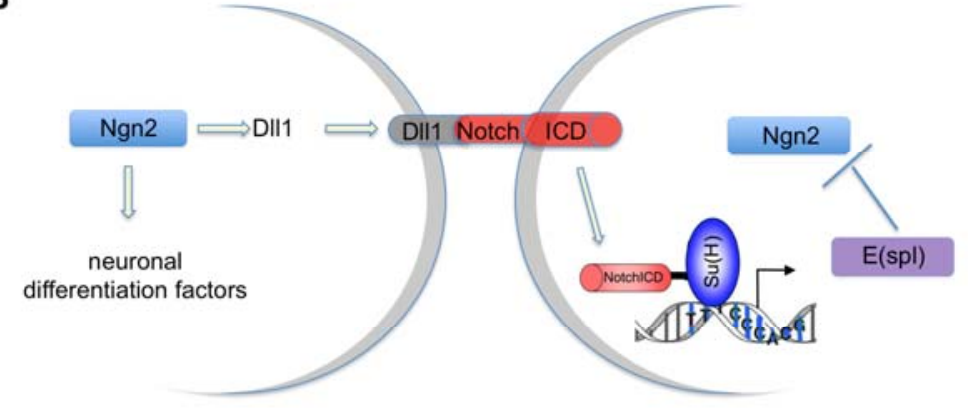

neuron

(signal-sending cell) undifferentiated cell (signal-receiving cell)
C

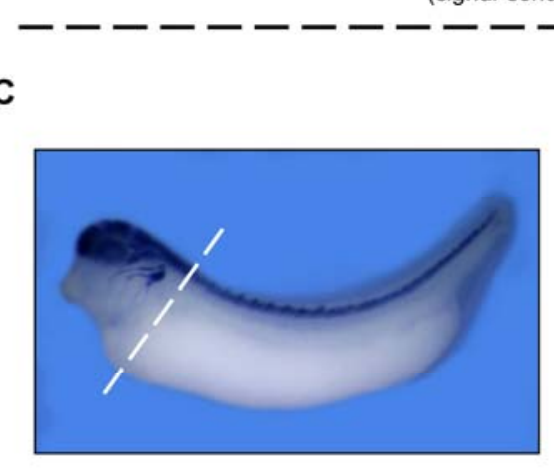

D

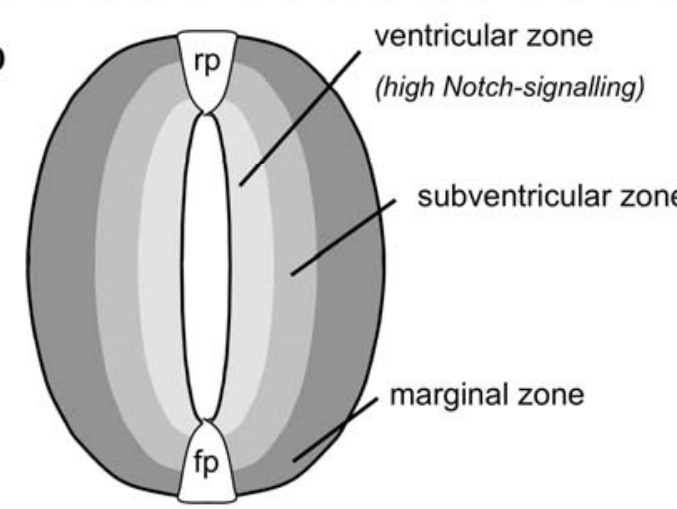

Figure 1.5 Notch-signalling during development.

(A, B) Lateral inhibition. At the open neural plate stage, the primary neurons arise in a salt-and-pepper like pattern, which is mediated by the Notch-signalling pathway. This process allows neuronal differentiation of a cell, whereas its neighboring cells stay in an undifferentiated cell state. Proneural genes such as Ngn2 activate neuronal differentiation factors as well as the Notch-ligand DIl1, which binds to the Notchreceptor at the neighboring cell. Upon binding, the Notch-receptor is proteolytic cleaved and the intracellular domain of the Notch-receptor is released into the nucleus. NotchICD binds $\mathrm{Su}(\mathrm{H})$ to form an activator complex to induce the transcription of the direct target genes. These transcription factors, in a negative-feedback loop, inhibit the expression of proneural genes. Thus the sending cell differentiates into a neuron, whereas the receiving cell stays in an undifferentiated cell state. (after Louvi and Artavanis-Tsakonas, 2006; Beres et al., 2006, modified) (C, D) Maintenance of neural progenitor cells. When the neural tube is closed, Notch-signaling is still essential as it is highly active in the ventricular zone of the neural tube and maintains the pool of progenitor cells. When cells start to differentiate into neurons, they migrate outwards through the subventricular zone into the marginal zone, where the differentiated and post-mitotic neurons are located. $r p$, roof plate; fp, floor plate

The direct targets of the Notch-signalling include members of the bHLH family that are related to the Drosophila Hairy and enhancer of split genes $(E(s p l)$ ) (Sasai et al., 1992; Ohtsuka et al., 1999; Davis and Turner, 2001). In a negative-feedback-loop, the Notch target genes act as repressors and inhibit the expression of the proneural genes (Takke et al., 1999; Schneider et al., 2001; Ishibashi et al., 1995; Cau et al., 2000) in the neighbouring cell (Fig. 1.5 B). Due to this mechanism, the ligand-expressing cell differentiates into a neuron, whereas the neighbouring cells stay in an undifferentiated cell state and 
later differentiate into glia-cells (Louvi and Artavanis-Tsakonas, 2006; Wang and Barres, 2000). In this context, overexpression of Notch-ICD in X. laevis decreases the number of primary neurons, while inhibition of the Notch-pathway

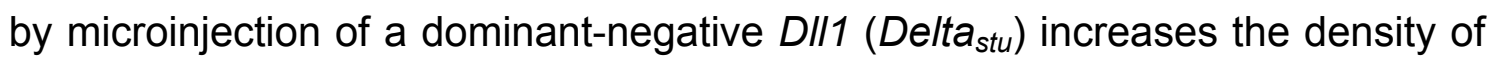
primary neurons (Chitnis et al., 1995). After neural tube closure, Notchsignalling is highly active in the ventricular zone of the neural tube and preserves the pool of neural progenitors (Lindsell et al., 1996; Imayoshi and Kageyama, 2011) (Fig. 1.5 C, D).

\subsection{Neurulation and Neuronal subtype specification}

During neurulation, the neural folds rise and fuse to form the closed neural tube. During this process, neural precursors cells migrate laterally inwards and reside in the ventricular zone (Fig. 1.5 D). After cell cycle exit, these precursor cells migrate outwards through the intermediate zone into the outer marginal zone (Fig. 1.5 D) (Bellefroid et al., 1996) and differentiate into distinct neuronal cell types. Neuronal subtype specification in vertebrates is best-characterised in the chick and mouse neural tube, in which the first neurons are born within the closed neural tube. Dorso-ventral patterning of the neural tube gives rise to specific neuronal subtypes within the neural tube. This is primarily generated through two signalling centers, BMP in the dorsal and Sonic Hedgehog $(\mathrm{SHH})$ in the ventral part of the neural tube (Fig. 1.6) (Dreau and Marti, 2012). These signals act as morphogens, generating gradients along the dorso-ventral axis of the embryo. Thus, the progenitor cells within the neural tube are exposed to different concentrations of these signals. Due to the partly opposing gradients, progenitors domains are generated in the inner ventricular zone of the neural tube. Each progenitor domain expresses distinct transcription factors, including bHLH and homeodomain (HD) transcription factors (Fig. 1.6) (Alaynick et al., 2011). From these progenitor domains, distinct neuronal subtypes arise that express a specific combinatorial code of HD transcription factors (Fig. 1.6) (Alaynick et al., 2011).

The roof plate and the dorsal ectoderm express several members of the TGF-beta-signalling pathway, including BMP-4, BMP-7, BMP-5 and Ds/1 (Liem et al., 1995; Liem et al., 1997). The importance and a concentration-dependent mechanism of the BMP-signals in generating dorsal interneuronal subtypes 
could be demonstrated by in vitro studies using neural plate explants as well as in vivo (Liem et al., 1997; Liem et al., 1995; Timmer et al., 2002; Barth et al., 1999; Nguyen et al., 2000). Furthermore, the in vitro experiments suggested that the different BMPs have qualitative distinct functions in generating the dorsal interneuron populations (Liem et al., 1997), which is further supported by in ovo electroporations in the chick neural tube (Le Dreau et al., 2012; Dreau and Marti, 2012)

In addition to BMP-signalling, the Wnt-pathway has been implicated in the dorsal patterning of the neural tube (Muroyama et al., 2002; Yu et al., 2008; Alvarez-Medina et al., 2008), which is supported by the expression of multiple Wnts in the roof plate and in the dorsal ectoderm (Parr et al., 1993; Hollyday et al., 1995; Megason and McMahon, 2002). Due to the dorsal extrinsic signalling events, six early ( $d \mathrm{l} 1-\mathrm{dl} 6)$ and two late $\left(\mathrm{d} I \mathrm{~L}_{\mathrm{A}}\right.$ and $\left.\mathrm{d} \mathrm{L}_{\mathrm{B}}\right)$ interneuron populations are generated (Fig. 1.6) (Helms and Johnson, 2003).

In the ventral neural tube, the morphogen $\mathrm{SHH}$ is expressed by the mesodermal derived notochord as well as by the floor plate induced by it (Echelard et al., 1993; Marti et al., 1995b; Placzek et al., 1991; Yamada et al., 1991). In vitro experiments showed a concentration-dependent induction of the ventral neuronal subtypes by SHH (Ericson et al., 1996; Ericson et al., 1997; Marti et al., 1995a; Roelink et al., 1995). However, of the five neuronal subtypes generated (V0-V3, MN) (Ericson et al., 1997; Briscoe et al., 2000), SHH is not sufficient for the specification of $\mathrm{VO}$ and $\mathrm{V} 1$ interneurons as in $\mathrm{SHH}$ knock-out mice, these neuron types are still present (Litingtung and Chiang, 2000).

The transcription factors in the neural tube can be divided into distinct subgroups. In the dorsal neural tube, genes can be divided into BMP-dependent (Class A) or -independent (Class B). Similarly in the ventral neural tube, Class I transcription factors are independent and in fact repressed by $\mathrm{SHH}$-signalling, whereas class II transcription factors are SHH-dependent (Briscoe and Ericson, 2001; Alaynick et al., 2011; Jessell, 2000). Furthermore, cross-repressive interactions between the distinct classes establish the borders of gene expression and thereby of the progenitor domains (Briscoe et al., 2000; Vallstedt et al., 2001). 


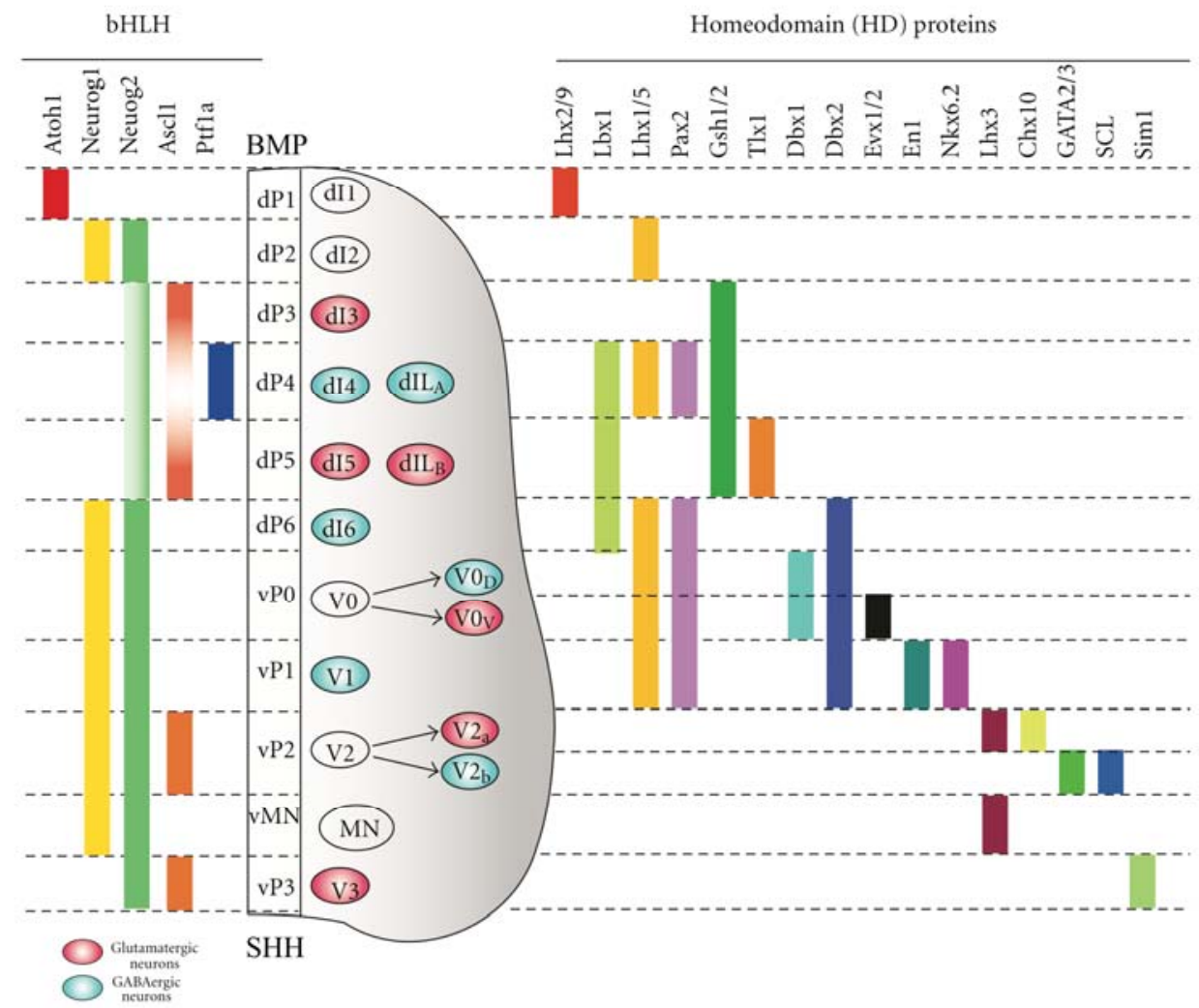

Figure 1.6 Dorso-ventral patterning of the mouse neural tube.

The patterning of the neural tube along the dorso-ventral axis is based on extrinsic signals. In the dorsal part, a gradient of BMP- and Wnt-signalling is generated by the roof plate and is responsible for the formation of the six early (dl1-dl6) and two late $\left(\mathrm{dlL}_{\mathrm{A}}\right.$ and $\mathrm{dl}_{\mathrm{B}}$ ) dorsal interneuronal subtypes. In the ventral part, the morphogen $\mathrm{SHH}$ is expressed from the notochord and the floor plate and is involved in the generation of the five ventral (V0-V3 and motor neurons (MN)) neuronal subtypes. The two ventral neuron populations $\mathrm{V} 0$ and $\mathrm{V} 2$ can be subdivided into two distinct neuron types (indicated by arrows). Due to the opposing gradients, specific bHLH (left) and HD (right) transcription factors become expressed in the ventricular zone along the dorsoventral axis, forming distinct neuronal progenitor domains. From these, different neuronal subtypes arise, which express a specific code of HD transcription factors. The neurotransmitter expression of the distinct post-mitotic neuron populations is indicated by red (glutamatergic) and blue (GABAergic) circles. (Hori and Hoshino, 2012, modified)

In $X$. laevis, the first neurons differentiate not within the closed neural tube, but already at the open neural plate stage in the three characteristic stripes on each side of the midline (Fig. 1.7 A). The region of the ectoderm that gives rise to the neural plate is excluded from BMP expression and can be visualized by the expression of Sox3, an early marker for neural progenitor cells (Fig. 1.7 A). However, the lateral stripe of primary neurons is located outside the Sox3 expression domain (Fig. 1.7 A) (Hardcastle and Papalopulu, 2000) and requires intermediate levels of BMP-signalling (Rossi et al., 2008). Furthermore, $\mathrm{SHH}$ is expressed in a longitudinal stripe along the dorsal midline (Brewster et 
al., 1998). Therefore, neuronal subtype specification in $X$. laevis may already be initiated at the open neural plate stage.

Dependant on their position within the open neural plate and the closed neural tube, the primary neurons obtain a specific neuronal subtype (Fig. 1.7 B). The lateral stripe of the primary neurons will give rise to the dorsal Rohon-Beard sensory neurons, while the intermediate and medial stripe will develop into interneurons and ventral motor neurons, respectively (Fig. 1.7 A and B) (Chitnis and Kintner, 1995; Hartenstein, 1989). The first differentiating neurons in the $X$. laevis spinal cord are the dorsal Rohon-Beard sensory neurons (Hartenstein, 1993) followed with a slight delay by the ventrally located neurons (Hartenstein, 1993), which include motor neurons and Kolmer-Agduhr cells. Interneurons are the last to differentiate and do so in the lateral part of the spinal cord (Hartenstein, 1993). A more detailed analysis considering soma location, axon projection and neurotransmitter expression defined in total ten different types of neurons in the $X$. laevis spinal cord (Roberts et al., 2012). These include the dorsal Rohon-Beard sensory neurons, the ventral motor neurons and KolmerAgduhr cells, but also seven distinct interneuronal subtypes (Roberts et al., 2012). In contrast to mouse, the molecular transcription factor codes to discriminate between distinct neuronal subpopulations are not well established. However, there is evidence indicating that these codes actually might be conserved in vertebrates.

A

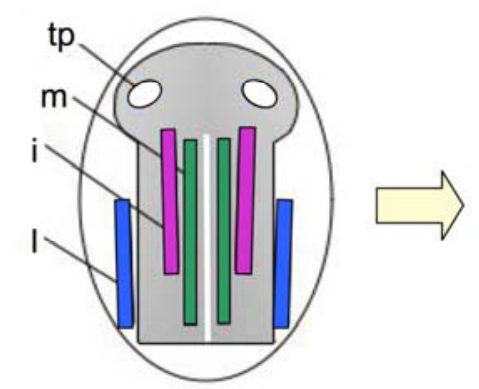

B

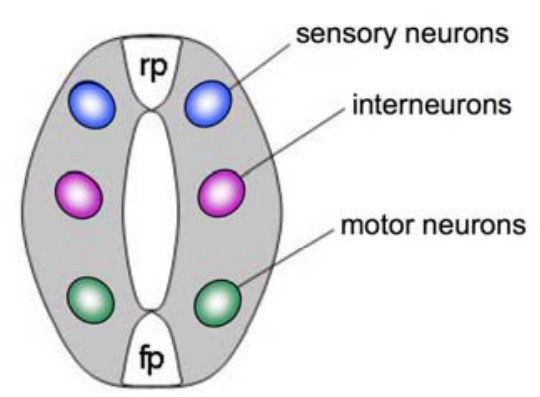

Figure 1.7 Neuronal subtype specification in the $X$. laevis spinal cord.

(A) Scheme of primary neurogenesis domains in $X$. laevis. At the open neural plate stage, primary neurons are born in three longitudinal stripes on each side of the midline (white) and in the trigeminal ganglia. Grey indicates Sox3 expressing areas, which reflect low BMP-signalling. (B) Scheme of neuronal subtype identity in the $X$. laevis spinal cord. When the neural tube is closed, the primary neurons obtain a specific neuronal subtype identity depending on their previous position within the neural plate. The outer stripe of primary neurons will give rise to the sensory Rohon-Beard neurons, while the medial and intermediate stripe will give rise to interneurons and motor neurons, respectively. $\mathrm{rp}$, roof plate, $\mathrm{fp}$, floor plate, $\mathrm{m}$, mediate, i, intermediate, I, lateral, tp, trigeminal placodes 


\subsection{Ptf1a}

The nervous system consists of many different cell types, including distinct neuronal subtypes, astrocytes and oligodendrocytes. Among the different neuronal cell types, most can be classified as excitatory or inhibitory neurons. An imbalance between the two distinct neuronal subtypes impairs functional neural networks, which underlies a number of neurological disorders (McCormick and Contreras, 2001; Rossignol, 2011). GABA and glutamate are the primary neurotransmitters of the inhibitory and excitatory neurons, respectively. The pancreatic transcription factor 1a (Ptf1a), which encodes for a bHLH transcription factor, has a central role in promoting GABAergic inhibitory neurons at the expense of glutamatergic excitatory neurons in the vertebrate CNS (Hori and Hoshino, 2012).

\subsubsection{Ptf1a function during development of the pancreas}

The importance of Ptf1a during embryogenesis is demonstrated by the pancreatic and cerebellar agenesis, as well as diabetes mellitus that occur in individuals with mutations in the Ptf1a gene (Sellick et al., 2004; Hoveyda et al., 1999; Tutak et al., 2009; Al-Shammari et al., 2011). A similar phenotype is observed upon loss of Ptf1a in the mouse (Kawaguchi et al., 2002; Sellick et al., 2004).

Ptf1a was first identified and characterised in the pancreas and was shown to be essential for the specification of pancreatic precursor cells including both, progenitors for endocrine and exocrine cells (Cockell et al., 1989; Burlison et al., 2008; Kawaguchi et al., 2002). Later in development, Ptf1a plays a central role in exocrine cell differentiation and in the adult exocrine pancreas, Ptf1a activates the transcription of the acinar digestive enzymes (Krapp et al., 1998; Krapp et al., 1996; Kawaguchi et al., 2002; Zecchin et al., 2004; Lin et al., 2004). Microinjection of Ptf1a mRNA in X. laevis is sufficient to induce ectopic formation of pancreatic tissue within the expression domains of the Pancreatic and duodenal homeobox $1(P d x 1)$ gene in the foregut (Afelik et al., 2006). Moreover, a combination of Pdx1 and Ptf1a allows conversion of non-pancreatic endodermal cells into both, endocrine and exocrine pancreatic cell lineages (Afelik et al., 2006).

$P d x 1$ was shown to be a direct target of Ptf1a in the mouse (Wiebe et al., 
2007; Miyatsuka et al., 2007) and additional Ptf1a downstream targets, including direct targets in early pancreatic progenitors (Thompson et al., 2012), in exocrine cell differentiation (Cockell et al., 1989; Masui et al., 2010) and in $X$. laevis have been identified (Bilogan and Horb, 2012). Additionally, Ptf1a itself has been reported to be a direct target through positive autoregulation (Masui et al., 2008). Experiments in zebrafish and mouse could show that the distinct Ptf1a activities during pancreas development are dosage-dependent. Low levels of Ptf1a expression promote pancreatic endocrine cell fates, whereas high levels specify exocrine cell differentiation (Fukuda et al., 2008; Dong et al., 2008). In addition, recent studies in the mouse showed that Ptf1a molecularly forms context-dependent transcription factor complexes. Ptf1a was originally identified as a subunit of the heterotrimeric pancreas transcription factor complex 1 (PTF1) (Cockell et al., 1989; Roux et al., 1989). In this complex, Ptf1a forms a heterodimer with an ubiquitously expressed class $\mathrm{A}$ bHLH factor (E-protein, E2A/p75/HEB/TCF12), which binds to the E-box sequence (CANNTG) at the DNA (Beres et al., 2006; Murre et al., 1989). Additionally, Ptf1a interacts with a member of the CSL family of DNA binding proteins $(\mathrm{Su}(\mathrm{H}), \mathrm{CBF} 1, \mathrm{Lag}-1, \mathrm{Rbp}-\mathrm{j}$ and $-\mathrm{I})$ through two conserved C-motifs (C1:HSLSW and C2:WTPEDPR) located in the C-domain of Ptf1a (Beres et al., 2006). The CSL-binding protein binds DNA via a TC-box motif (TTTCCCA) (Beres et al., 2006). Thus, the heterotrimeric PTF1 complex binds a specific bipartite binding site, in which the TC-box must be separated by one or two helix turns from the E-box motif (Cockell et al., 1989). In pancreatic progenitor cells, Ptf1a interacts with Rbp-j, which at the onset of acinar cell development is gradually replaced by its paralogue Rbp-I (Masui et al., 2007). Strong expression of Rbp-I is restricted to adult pancreatic tissue and is so far unknown in $X$. laevis (Beres et al., 2006).

In the mouse, the interaction between Ptf1a and Rbp-j/-I, but not with the class A bHLH protein can be abolished by introducing mutations in the $\mathrm{C} 1$ and C2 domain of Ptf1a (Beres et al., 2006). Rbp-j, but not Rbp-I is also involved in Notch-signalling, where it forms a transcriptional complex with Notch-ICD. It could be demonstrated that the binding of Rbp-j to Notch-ICD and Ptfla is mutually exclusive (Beres et al., 2006). Thus, Ptf1a and Notch-ICD activity could potentially antagonize each other. Furthermore, Notch target genes can 
directly bind to Ptf1a (Ghosh and Leach, 2006) and downregulate Ptf1a transcriptional activation and DNA-binding (Esni et al., 2004).

\subsubsection{Ptf1a function during development of the nervous system}

In the vertebrate nervous system, Ptf1a is expressed in the retina, the hindbrain and in the dorsal horn of the spinal cord in precursor cells of GABAergic inhibitory interneurons (Glasgow et al., 2005; Hoshino et al., 2005; Nakhai et al., 2007; Fujitani et al., 2006). Ptf1a was shown to act as a selector gene for GABAergic inhibitory interneurons at the expense of glutamatergic excitatory neurons in the vertebrate retina, hindbrain and spinal cord (Glasgow et al., 2005; Hori et al., 2008; Hoshino et al., 2005; Fujitani et al., 2006; Nakhai et al., 2007; Pascual et al., 2007; Dullin et al., 2007). Consistent with the molecular mechanism of gene activation in the pancreas, Ptf1a in neural tissue forms a heterotrimeric PTF1 complex through interaction with Rbp-j and the ubiquitous expressed class A bHLH transcription factor. For its function as a GABAergic selector gene, Ptf1a requires the interaction with Rbp-j (Hori et al., 2008; Lelievre et al., 2011) and Rbp-j function in this context is Notchindependent (Hori et al., 2008).

In contrast to the pancreas, only a few direct downstream targets of Ptf1a in the nervous system have been identified. Similar to the pancreas, Ptf1a regulates its own expression via positive autoregulation (Meredith et al., 2009). In the mouse neural tube and cerebellum, the bHLH transcription factor Ngn2 is a direct downstream target of the heterotrimeric PTF1 complex (Henke et al., 2009b). Furthermore, the expression of two members of the immunoglobulin superfamily, Nephrin and Neph3 (Kirre/2 in X. laevis) is directly controlled by binding of Ptf1a within the promoter region of these genes (Nishida et al., 2010). Neph3 is expressed in progenitors of the GABAergic Purkinje cells, suggesting that its activation by Ptf1a plays a role in GABAergic neuronal subtype specification (Mizuhara et al., 2010). In combination with the oligodendrocyte transcription factor 3 (Olig3), Ptf1a induces the expression of the forkhead box D3 (FoxD3) gene to generate the glutamatergic climbing fiber neurons (Yamada et al., 2007; Storm et al., 2009). 


\subsubsection{Ptf1a also forms context-dependent transcription complexes in the nervous system}

In X. laevis, Ptf1a not only functions as a selector gene for GABAergic inhibitory neuronal cell fates, but also promotes general neurogenesis in naïve ectodermal explants and in the non-neural ectoderm of embryos (Dullin et al., 2007; Hedderich, 2008). Analysis of a limited set of marker genes demonstrated that Ptf1a drives neuronal differentiation similar to the neuronal determination gene Ngn2 (Hedderich, 2008). However, Ptf1a and Ngn2 have distinct neuronal subtype specificities (Dullin et al., 2007; Hedderich, 2008). Ptf1a activates the expression of Glutamate decarboxylase 1 (Gad1), a marker for GABAergic inhibitory neurons (Li et al., 2006). In contrast, Ngn2 induces the expression of Hox11L2, which marks sensory neurons (Patterson and Krieg, 1999; Perron et al., 1999). The murine homolog of Hox11L2, Tx/3 functions as a selector gene for glutamatergic excitatory neurons (Cheng et al., 2004; Cheng et al., 2005). Mutations in the $X$. laevis Ptf1a C1- and C2-domain $\left(\mathrm{Ptf}_{1} \mathrm{a}^{\mathrm{W} 224 \mathrm{~A} / \mathrm{W} 242 \mathrm{~A}}\right)$ reveal that the interaction of Ptf1a with $\mathrm{Su}(\mathrm{H})$ is essential for the GABAergic specificity of Ptf1a (Hedderich, 2008), a result congruent to the situation in the murine nervous system (Hori et al., 2008). Surprisingly, the proneural activity of Ptf1a was not impaired by the mutations, as Ptf1a $\mathrm{a}^{\text {W224A/W242A }}$ could still activate neuronal differentiation genes (Hedderich, 2008). Taken together, the data suggest that Ptf1a also forms context-dependent transcription complexes in the nervous system: $A \mathrm{Su}(\mathrm{H})$-independent complex to drive general neurogenesis and a $\mathrm{Su}(\mathrm{H})$-dependent complex to specify GABAergic inhibitory neurons (Hedderich, 2008) (Fig. 1.8). 

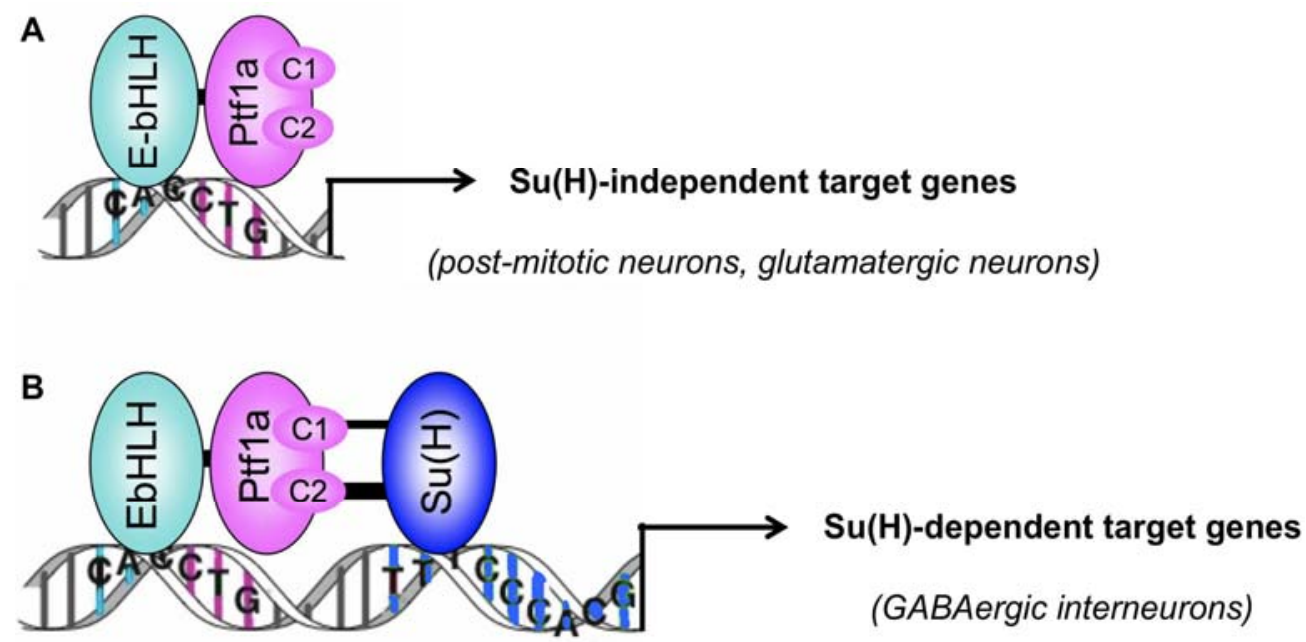

Figure 1.8 Ptf1a forms context-dependend transcription complexes

(A) In the context of general neurogenesis, the dimeric complex, consisting of Ptf1a and the class A bHLH transcription factor is sufficient to drive neuronal differentiation and to induce the formation of glutamatergic/sensory neurons. Thus, this complex activates $\mathrm{Su}(\mathrm{H})$-independent Ptf1a target genes. (B) In contrast, the genes involved in GABAergic interneuron formation are $\mathrm{Su}(\mathrm{H})$-dependent as the $\mathrm{Su}(\mathrm{H})$-interaction of Ptf1a is essential for their activation. (Beres et al., 2006, modified)

\subsection{Aims}

The bHLH transcription factor Ptf1a was identified as an important selector gene for generation of GABAergic inhibitory neurons in the vertebrate CNS. However, previous experiments in $X$. laevis indicated that Ptf1a also functions as a proneural gene and forms context-dependent transcription complexes to drive neuronal differentiation and to promote GABAergic inhibitory interneurons. The goal of the thesis was to further analyse the two distinct Ptf1a specificities, including identification of the genetic network downstream of Ptf1a and providing insight into the regulation of the distinct transcription complexes. 


\section{Materials and Methods}

\subsection{Material}

\subsubsection{Model organism}

The African clawed frog Xenopus laevis ( $X$. laevis) was used as a model organism during this study. Albinos and pigmented frogs were obtained from Nasco (Ft. Atkinosn, USA). The embryos were staged according to Nieuwkoop and Faber (1967).

\subsubsection{Bacteria}

For molecular biology standard methods, the chemical competent Escherichia coli strain XL1-Blue was used.

XL1-Blue: recA1, endA1, gyrA96, thi-1, hsdR17, supE44, relA1, lac[F'proAB, laclqZDM15, Tn10(Tetr)]c (Stratagene).

\subsubsection{Antiobiotics and Media}

\section{Antibiotics}

Ampicillin

stock: $100 \mathrm{mg} / \mathrm{ml}$ in $\mathrm{dH}_{2} \mathrm{O}$, working: $100 \mu \mathrm{g} / \mathrm{ml}$. Stored at $-20^{\circ} \mathrm{C}$.

\section{Media}

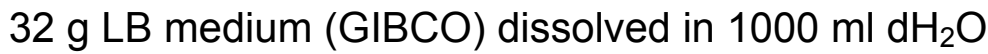

The LB Media was autoclaved for $20 \mathrm{~min}$ and cooled down to $50{ }^{\circ} \mathrm{C}$. Afterwards the selective antibiotic was added. The agar plates were poured under a steril bench and stored at $4{ }^{\circ} \mathrm{C}$.

\subsubsection{Oligonucleotides}

Oligonucleotides were purchased from Sigma-Aldrich and dissolved in $\mathrm{HPLC} \mathrm{H}_{2} \mathrm{O}$ to $100 \mu \mathrm{M}$ or $500 \mu \mathrm{M}$. 


\section{Sequencing oligonucleotides}

Table 2.1 Summary of Sequencing oligonucleotides and annealing temperature $\left(\mathrm{T}_{\mathrm{A}}\right)$

\begin{tabular}{|l|l|r|}
\hline Oligonucleotide & \multicolumn{1}{|c|}{ Sequence } & $\mathbf{T}_{\mathbf{A}}{ }^{\circ} \mathbf{C}$ \\
\hline SP6 & 5'-TTAGGTGACACTATAGAATAC-3' & 48 \\
\hline T7 & 5'-TAATACGACTCACTATAGGGCGA-3' & 56 \\
\hline T7 pcs2+ & 5'-TCTACGTAATACGACTCACTATAG-3' & 56 \\
\hline GR7 & 5'-ATCCTGCATATAACACTTC & 56 \\
\hline T3 & 5'-AATTAACCCTCACTAAAGGG-3' & 57 \\
\hline
\end{tabular}

\section{RT-PCR oligonucleotides}

Table 2.2 Summary of RT-PCR oligonucleotides and working conditions

\begin{tabular}{|c|c|c|c|c|}
\hline Oligonucleotide & Orientation & Sequence & $\mathrm{T}_{\mathrm{A}}{ }^{\circ} \mathrm{C}$ & Cycles \\
\hline $\mathrm{H} 4$ & for & 5'-CGGGATAACATTCAGGGTATCACT-3' & 56 & 24 \\
\hline $\mathrm{H} 4$ & rev & 5'-ATCCATGGCGGTAACTGTCTTCCT-3' & 56 & 24 \\
\hline ODC & for & 5'-GCCATTGTGAAGACTCTCTCCATTC -3' & 56 & 24 \\
\hline ODC & rev & 5'-TTCGGGTGATTCCTTGCCAC-3' & 56 & 24 \\
\hline $\mathrm{N}$-tubulin & for & 5'ACACGGCATTGATCCTACAG-3' & 57 & 30 \\
\hline $\mathrm{N}$-tubulin & rev & 5'AGCTCCTTCGGTGTAATGAC-3' & 57 & 30 \\
\hline Gad1 & for & 5'-ATGGGCGTCTTACTCCAATG-3' & 60 & 33 \\
\hline Gad1 & rev & 5'-ATGTCTACATGGCGACCACA-3' & 60 & 33 \\
\hline Hox11L2/TIx3 & for & 5'-GCCAACAAGTACAAGTGCACAG-3' & 57 & 30 \\
\hline Hox11L2/TIx3 & rev & 5'-CAGGAGCCAGACTCACATTGAC-3' & 57 & 30 \\
\hline $\mathrm{Su}(\mathrm{H})$ & for & 5'-AGAAGGTTGGAGATGGGTTC-3' & 60 & 33 \\
\hline $\mathrm{Su}(\mathrm{H})$ & rev & 5'-GATGATGTGACATTGGCTGA-3' & 60 & 33 \\
\hline Ptf1a 3'UTR & for & 5'-GTTGTCAGAACGGCCAAAGT-3' & 60 & 33 \\
\hline Ptf1a 3'UTR & rev & 5'-GGTACCGAGTGGAACCAAAG-3' & 60 & 33 \\
\hline Ngn2 5'UTR & for & 5'-ACTGCAGCATTGTCACTTGC-3' & 60 & 34 \\
\hline Ngn2 5'UTR & rev & 5'-CAATGGTTAGCCCCAATGTT-3' & 60 & 34 \\
\hline
\end{tabular}

\subsubsection{Overexpression constructs}

Ptf1a-GRpCS2+ (Afelik et al., 2006), Ptf1a-VP16-GRpCS2+ (Dullin et al., 2007), GR-Ngn2-p3' (Perron et al., 1999), ß-Gal-pCS2+ (Chitnis et al., 1995).

Ptf1a W224A/W242A - GRpCS2+ (Hedderich, 2008). This construct harbors the open reading frame of Ptf1a (DQ007931.1) with two point mutations at position 224 and 242. The point muations were introduced using the "QuickChange XL Site directed Mutagenesis" kit (Stratagene) and the following primers p48C1mut_up GGA CAT TCT CTC TCA GCG ACT GAT GAG AAG CAA CTG AG and p48C1mut_down CTC AGT TGC TTC TCA TCA GTC GCT GAG AGA GAA TGT CC as well as p48C2mut_up GTT GTC AGA ACG GCC AAA GTG GCG 
ACT CCT GAG GAT CC and p48C2mut_down GGA TCC TCA GGA GTC GCC ACT TTG GCC GTT CTG AC AAC, thereby exchanging a tryptophan to an alanine (mutations are red). Ptf1a-GRpCS2+ (Afelik et al., 2006) served as DNA template for the PCR. For preparation of sense mRNA, the construct was linearized with Notl and transcribed with SP6 polymerase.

Chimeric Ptf1a-GRpCS2+ (Hedderich, 2008). To synthesize the chimric Ptf1aGR, the bHLH domain of Ngn2 was PCR amplified using GR-Ngn2p3' (Perron et al., 1999) as template. The primers contained the surrounding sequences of the bHLH domain of Ptf1a (in italics): Ptf1a/bHLH-Ngnr1_for CTG AGG TCG GAC GCG GAG ATG CAG CAG CGG CGC GTT AAA GCT AAC AAC and Ptf1a/bHLH-Ngnr1_rev GCG GCA GAT CGG ACT GTA CCA TCT CGC TAA GAG CCC AGA TGT AGT TGT AG. The so called megaprimer was used together with the "QuickChange XL Site Directed Mutagenesis" kit (Stratagene) in a PCR using Ptf1a-GRpCS2+ (Afelik et al., 2006) as template. For preparation of sense mRNA, the construct was linearized with Notl and transcribed with SP6 polymerase.

Su(H)-HApCS2+ (Hedderich, 2008). This construct harbors the open reading frame of $\mathrm{Su}(\mathrm{H})$ (U60093.1), which was PCR amplified using $\mathrm{Su}(\mathrm{H}) \mathrm{pCS} 2+$ (Wettstein et al., 1997) as a template as well as the following primers: 3'HASu(H)_BamHI_for: CTG GAT CCA TGC AAC CTG GC and 3'HASu(H)_Xhol_rev: CAA CTC GAG GGA CAC TAC TGC TGC. Su(H) was subcloned into HA-pCS2+ (Damianitsch, 2008) using the BamHI und Xhol restriction sites. For preparation of sense mRNA, the construct was linearized with Notl and transcribed with SP6 polymerase.

Su(H)-GRpCS2+. The open reading frame of $\mathrm{Su}(\mathrm{H})$ (U60093.1) was PCR amplified with the following primers: $\mathrm{Su}(\mathrm{H})$ _BamHI_for: CGG GAT CCA TGC AAC CTG GCA TTC and Su(H)_BamHI_rev: CGG GAT CCG GGG ACA CTA CTG CTG and using $\mathrm{Su}(\mathrm{H})-\mathrm{HApCS} 2+$ as template. $\mathrm{Su}(\mathrm{H})$ was subcloned into GRpCS2+ (D. Turner and R. Rupp derivate) using the BamHI restriction sites. For preparation of sense mRNA, the construct was linearized with Notl and transcribed with SP6 polymerase. 
MT-Ptf1a-GRpCS2+. The open reading frame of Ptf1a (DQ007931.1) was PCR amplified using the primers: Ptf1a_EcoRI_for: CGG AAT TCC ATG GAA ACG GTC C and Ptf1a_Stul_rev: GAA GGC CTC ATA TCA AGG CAC AAA GT. Ptf1a-GRpCS2+ was used as template in the PCR. The amplified Ptf1a was subcloned into MT-GRpCS2+ (D. Turner and R. Rupp derivate) uisng the EcoRI and Stul restrictions sites. For preparation of sense mRNA, the construct was linearized with Notl and transcribed with SP6 polymerase.

MT-Ptf1a ${ }^{\text {W224A/W242A }}-$ GRpCS2+. The open reading frame of Ptf1a ${ }^{\text {W224A/W242A }}$ was PCR amplified with the following primers: Ptf1a_EcoRI_for: CGG AAT TCC ATG GAA ACG GTC C and Ptf1a_Stul_rev: GAA GGC CTC ATA TCA AGG CAC AAA GT. Ptf1a ${ }^{\text {W224A/W242A }}-$ GRpCS2+ served as template in the PCR. The amplified Ptf1a W224A/W242A was subcloned into MT-pCS2+GR (D. Turner and R. Rupp derivate) uisng the EcoRI and Stul restrictions sites. For preparation of sense mRNA, the construct was linearized with Notl and transcribed with SP6 polymerase.

\subsubsection{Constructs for real-time RT-PCR standard curves}

To perform standard curves in the real-time RT-PCR, the amplified RT-PCR products were subcloned into the pGEM-T easy vector (Promega). The amplified region is given with respect to the ATG.

Gad1pGEMT easy (U38225, 1126 to 1289); ODCpGEMT easy (X56316, 222 to 441); N-tubulinpGEMT easy (X15798, 80 to 329); Hox11L2/TIx3pGEMT easy (AF283693, 9 to 274 ) 


\subsection{7 antisense RNA Constructs}

Table 2.3 Summary of antisense RNA constructs

\begin{tabular}{|l|l|l|l|l|}
\hline Name & Vector & Restriction enzyme & Polymerase & Reference \\
\hline N-tubulin & pBluescriptst KS & BamHI & T3 & Chitnis et al., 1995 \\
\hline Lbx1 & pCS107 & Sall & T7 & Martin and Harland, 2006 \\
\hline Lim1/Lhx1 & pBluescriptst-KS & Xhol & T7 & Taira et al. 1994 \\
\hline Gad1 & pBluescript SK+ & BamHI & T7 & Li et al., 2006 \\
\hline Hox11L2 & BstEII & Stul & T3 & Patterson and Krieg, 1999 \\
\hline Pax2 & pT7TS & EcoRI & T3 & - \\
\hline Lmx1b & pGEM-T & Sall & T7 & Haldin et al., 2003 \\
\hline Ngn1 & pCS2P+ & EcoRI & T7 & Nieber et al., 2009 \\
\hline Ngn2 & pBluescript II & BamHI & T3 & Ma et al., 1996 \\
\hline Ngn3 & pCS2P+ & EcoRI & T7 & Nieber et al., 2009 \\
\hline Ptf1a & pGEM-T & Notl & T7 & Chen et al., 2004 \\
\hline Ptf1a sense & pGEM-T & Spel & SP6 & \\
\hline
\end{tabular}

\subsection{Methods}

\subsubsection{DNA standard methods}

\subsubsection{DNA restriction digestion}

Restriction digests were performed according to the manufacturer's instruction using restriction enzymes purchased from Fermentas Life Sciences.

\subsubsection{Agarose gel electrophoresis}

TAE (Tris/Acetate/EDTA): 40 mM Tris-Acetate, $\mathrm{pH}$ 8.5, 2 mM EDTA

Analytical and preparative restrictions or PCR products were analysed by agarose gel electrophoresis (Sharp et al., 1973). Depending on the size of the DNA fragments, different percentages of agarose gels were used $(0.7 \%$ to $2 \%)$. The gels were made out of agarose and 1x TAE buffer, which was also used as running buffer. To analyse the size of the DNA fragments, Standard DNA ladders (FastRuler DNA Ladder, Fermentas) were used. For visualization of the DNA, ethidium bromide $(0.5 \mu \mathrm{g} / \mathrm{ml})$ was added and for documentation the ChemiDoc video documentation system (EASY view) was used.

\subsubsection{Purification of DNA fragments and linearized templates}

Purification of DNA fragments from agarose gels or restriction digestions were performed with the "Invisorb Fragment Cleanup" kit (Invitek) or the "GFX 
PCR DNA und Gel Band Purification" kit (GE Healthcare) according to the manufacturer's instructions.

\subsubsection{Polymerase chain reaction (PCR)}

A standard PCR reaction (Mullis et al., 1986) contained 1x Polymerase buffer, $0.2 \mathrm{mM}$ of each dNTP (Thermo Scientific), $1.15 \mathrm{mM}$ of each oligonucleotide and 0.5-1 U Pfu Polymerase (Stratagene $\mathrm{GmbH}$ ) as well as 100 ng template DNA. The cycle numbers of the PCR reaction were adapted to the product size and the temperature adapted to the melting temperature of the oligonucleotides. The PCR reaction was performed in thermocyclers (Biometra) with the following program: $95^{\circ} \mathrm{C} 2 \mathrm{~min}$., $95^{\circ} \mathrm{C} 45 \mathrm{sec}$., $\mathrm{Tm}-2{ }^{\circ} \mathrm{C} 45 \mathrm{sec}$., $72{ }^{\circ} \mathrm{C}$ $60 \mathrm{sec}$ per $1 \mathrm{~kb}$ product, $72{ }^{\circ} \mathrm{C} 600 \mathrm{sec}, 8^{\circ} \mathrm{C}$ Pause.

\subsubsection{DNA ligation}

Standard ligation reactions were carried out using the T4 DNA Ligase (10 $\mathrm{U} / \mu \mathrm{l}$ ) (Fermentas Life Sciences) according to the manufacturer's instructions. The ligation was incubated overnight at room temperature (RT). To prevent selfreligation, the linearised vector was treated with $1 \mathrm{U}$ CIAP before ligation.

\subsubsection{Chemical transformtaion of bacteria cells}

For chemical transformation of the ligation reaction, the chemical competent cells XL1-Blue were used. $100 \mu$ to $200 \mu$ l thawed cells were added to the ligation and incubated on ice for $30 \mathrm{~min}$. After incubation, a heat shock at $42{ }^{\circ} \mathrm{C}$ for $90 \mathrm{sec}$ was performed followed by a further incubation on ice for 3 $\min .800 \mu$ LB medium was added and the reaction was incubated for $45 \mathrm{~min}$ at $37{ }^{\circ} \mathrm{C}$ with mild shaking at $300 \mathrm{rpm}$. After centrifugation for $30 \mathrm{sec}$ at 10,000 rpm, $800 \mu \mathrm{l}$ supernatant was discarded and the bacteria pellett was resuspended in the remaining media. The bacteria solution was seeded on LB agar plates supplemented with the appropriate antibiotic for the selection of the transformed cells. Colonies were grown over night at $37{ }^{\circ} \mathrm{C}$ (Mandel and Higa 1970). 


\subsubsection{Plasmid DNA preparation}

The bacteria were grown in $4 \mathrm{ml}$ LB media together with the selective antibiotic overnight at $37{ }^{\circ} \mathrm{C}$ with shaking at $250 \mathrm{rpm}$. For the plasmid preparation, $3 \mathrm{ml}$ of the bacteria culture was centrifuged for $2 \mathrm{~min}$ at 13,000 rpm. Isolation of DNA in analytical amounts was performed using the "GeneJETтм Plasmid Miniprep" kit (Fermentas) according to the manufacturer's protocol. Isolation of DNA in preparative amounts was carried out with the "NucleoBond ${ }^{\circledR}$ Xtra Midi" kit (Macherey-Nagel) following the manufacturer's protocol. The DNA concentration was measured with the NanoDrop-2000c spectrophotometer (Thermo Scientific) using $1 \mu$ of the DNA sample.

\subsubsection{DNA-sequencing}

Sequencing of plasmid DNA (Sanger et al., 1977) was performed with the "Big Dye Terminator Cycle Sequencing" kit (Applied Biosystems) according to the manufacturer's protocol. The reaction was performed in a thermocycler with the following PCR program: $95^{\circ} \mathrm{C} 2 \mathrm{~min}, 95^{\circ} \mathrm{C} 30 \mathrm{sec}$., $55^{\circ} \mathrm{C} 30 \mathrm{sec}$., 60 ${ }^{\circ} \mathrm{C} 4 \mathrm{~min}, 12{ }^{\circ} \mathrm{C}$ pause. In total, 26 cycles from step two to four were performed.

To purify the sequencing reaction, $1 \mu \mathrm{IEDTA}(125 \mathrm{mM}), 1 \mu \mathrm{NaAc}(3 \mathrm{M})$ and $50 \mu \mathrm{l} 100 \%$ Ethanol were added and incubated for $5 \mathrm{~min}$ at RT. After centrifugation for $20 \mathrm{~min}$ at $13,000 \mathrm{rpm}$, the pellet was washed with $70 \%$ ethanol and centrifuged for 5 min with 13,000 rpm. The pellet was air-dried and resuspended in $15 \mu \mathrm{l} \mathrm{HiDi.} \mathrm{To} \mathrm{sequence} \mathrm{the} \mathrm{reaction,} \mathrm{the} \mathrm{ABI} 3100 \mathrm{xl}$ Genetic Analyzer (Applied Biosystems) was used.

\subsubsection{RNA techniques}

\subsubsection{In vitro synthesis of capped sense mRNA}

In vitro capped sense mRNA was synthesized using the "SP6/T3 mMESSAGE mMACHINE" kit (Ambion Inc.) according to the manufacturer's protocol. For a $20 \mu \mathrm{l}$ reaction, $1 \mu \mathrm{g}$ of linearised plasmid was used. The purification of the synthesized mRNA was performed using the "Illustra RNASpin Mini" kit (GE Healthcare) according to the manufacturer's instructions. The RNA was eluted in $30 \mu \mathrm{l}$ RNase-free water at $80{ }^{\circ} \mathrm{C}$. The RNA concentration was measured using the NanoDrop 2000c spectrophotometer 
(Thermo Scientific). The quality of the mRNA was analysed on a $1 \%$ agarose gel. The RNA was aliquoted in $2 \mu$ and stored at $-80^{\circ} \mathrm{C}$.

\subsubsection{In vitro synthesis of antisense RNA}

Antisense RNA was used in the whole mount in situ hybridization as probe to detect in vivo transcripts. To synthesize antisense RNA, $1 \mu \mathrm{g}$ of the linearised plasmid was used in a $25 \mu$ reaction. A standard reaction contained:

$5 \mathrm{x}$ transcription buffer (Fermentas)

ATP, GTP, CTP, UTP, Dig or Flu-UTP (10 mM each)

DTT $(0.75 \mathrm{M})$

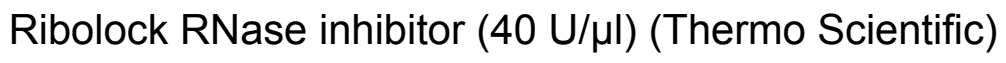

RNA polymerase $(20 \mathrm{U} / \mu \mathrm{l})$ (Fermentas)

The reaction was incubated for two hours at $37^{\circ} \mathrm{C}$. Afterwards, the DNA

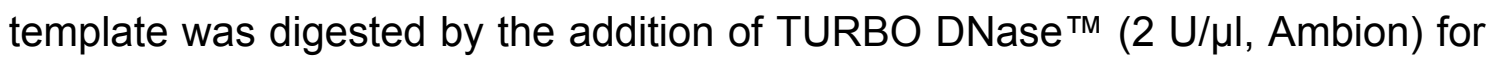
$30 \mathrm{~min}$ at $37^{\circ} \mathrm{C}$. The purification of the antisense RNA was performed using the "RNeasy ${ }^{\circledR}$ Mini" kit (Qiagen $\mathrm{GmbH}$ ) according to the manufacturer's protocol. The RNA was eluted in $100 \mu \mathrm{l}$ RNase-free water at $80^{\circ} \mathrm{C}$. The quality of the antisense RNA was analysed on a $1 \%$ agarose gel. $1 \mathrm{ml}$ of hybridization mix was immediately added to the antisense RNA and stored at $-20^{\circ} \mathrm{C}$.

\subsubsection{RNA isolation from ectodermal explants ("animal caps")}

For total RNA isolation, 50 to 100 ectodermal explants were cut, frozen in liquid nitrogen and stored at $-80{ }^{\circ} \mathrm{C}$.

Total RNA isolation was performed using the "RNAqueous®-Micro" kit (Ambion) or trizol as described below. After RNA isolation according to both protocols, the RNA quality was evaluated with the 2100 Bioanalyzer (Agilent). To check for genomic DNA contamination, a control PCR with histone $\mathrm{H} 4(\mathrm{H} 4)$ was performed and analysed on a $2 \%$ agarose gel.

\subsection{Isolation of total RNA with the "RNAqueous@-Micro" kit (Ambion)}

The animal caps were lysated in $100 \mu$ lysis buffer by a 29-Gauge syringe and centrifuged for $5 \mathrm{~min}$ at $4{ }^{\circ} \mathrm{C}$. Afterwards, total RNA was isolated 
according to the manufacturer's protocol. The RNA was eluted in $30 \mu$ l elution buffer at $75{ }^{\circ} \mathrm{C}$. For genomic DNA digestion, the RNA was incubated with DNasel provided within the kit for $1.5 \mathrm{~h}$ at $37{ }^{\circ} \mathrm{C}$. The DNAsel activity was inhibited by incubation for $10 \mathrm{~min}$ at $70{ }^{\circ} \mathrm{C}$ followed by the use of the DNasel inactivation reagent according to the manufacturer's protocol.

\subsection{Isolation of total RNA with trizol}

The animal caps were lysated in $400 \mu \mathrm{l}$ trizol with a 29-Gauge syringe and vortexed for $30 \mathrm{sec} .80 \mu \mathrm{l}$ chloroform was added, vortexed and centrifuged for $10 \mathrm{~min}$ at $4{ }^{\circ} \mathrm{C}$ and $13,000 \mathrm{rpm}$. After centrifugation, the upper phase, containing the total RNA (approximately $200 \mu \mathrm{l}$ ), was transferred to a new eppendorf tube. $200 \mu \mathrm{l}$ chlorofom was added, vortexed for $30 \mathrm{sec}$ and again centrifuged for $10 \mathrm{~min}$ at $4{ }^{\circ} \mathrm{C}$. The supernatant was recovered and transferred into a new eppendorf tube and $500 \mu$ isopropanol was added, mixed well and incubated at $-20{ }^{\circ} \mathrm{C}$ for at least $2 \mathrm{~h}$. After centrifugation at $4{ }^{\circ} \mathrm{C}$ and $13,000 \mathrm{rpm}$ for $30 \mathrm{~min}$, the precipitate was washed with $400 \mu \mathrm{l}$ of $70 \%$ ethanol and centrifuged for $2 \mathrm{~min}$ at $4{ }^{\circ} \mathrm{C}$. The pellet was air-dried and resolved in $30 \mu \mathrm{l}$ RNase-free water. For genomic DNA digestion, the RNA was incubated with DNasel $(1 \mathrm{U} / \mu \mathrm{l})\left(\right.$ Thermo Scientific) at $37^{\circ} \mathrm{C}$ for $1.5 \mathrm{~h}$. The DNAsel activity was inhibited by incubation for $10 \mathrm{~min}$ at $70^{\circ} \mathrm{C}$.

\subsubsection{Reverse transcription and PCR (semi-quantitative and quantitative RT-PCR)}

\subsection{Reverse transcription}

For cDNA sysnthesis, $50 \mathrm{ng}$ to $100 \mathrm{ng}$ total RNA was used per $10 \mu \mathrm{l}$ reaction mix. A standard reaction mix contained $5 \mathrm{mM} \mathrm{MgCl} 2,2.5 \mathrm{ng}$ random hexamer, $5 \mathrm{mM}$ dNTP mix, $8 \mathrm{U}$ Ribolock RNase inhibitor (Thermo Scientific) and $20 \mathrm{U}$ MuLV reverse transcriptase (Roche) in 1x Go Taq flexi buffer (Promega). The synthesis was performed in cycler using the following conditions: $20{ }^{\circ} \mathrm{C}$ for $20 \mathrm{~min}, 42^{\circ} \mathrm{C}$ for $1 \mathrm{~h}$ and $95^{\circ} \mathrm{C}$ for $5 \mathrm{~min}$. 


\subsection{Semi-quantitative RT-PCR}

For semi-quantitative RT-PCR analysis, $2.5 \mu \mathrm{l}$ cDNA was added to a 12.5 $\mu \mathrm{l}$ total reaction volume. The standard reaction mix contained: 1x Go Taq reaction buffer (Promega), $0.2 \mathrm{mM}$ of RT-primer each as well as $0.5 \mathrm{U}$ Go-Taq DNA-Polymerase (Promega). The reaction was performed in a thermocycler with the following program: $2 \mathrm{~min}$ at $95{ }^{\circ} \mathrm{C}, 45 \mathrm{sec}$ at $95{ }^{\circ} \mathrm{C}, 45 \mathrm{sec}$ at annealingtemperature of the oligonucleotides, $45 \mathrm{sec}$ at $72{ }^{\circ} \mathrm{C}, 5 \mathrm{~min}$ at $72{ }^{\circ} \mathrm{C}$. The cycle number from step 2 to 5 of the PCR was adapted to the oligonucleotides. The RT-PCR was analysed on a $2 \%$ agarose gel.

\subsection{Quantitative real-time RT-PCR}

For quantitative real-time RT-PCR analysis, $5 \mu \mathrm{l}$ cDNA was added to a $25 \mu \mathrm{l}$ reaction volume. The standard reaction mix contained: $1 \mathrm{x}$ $\mathrm{iQ}^{\mathrm{TM}}$ SYBRGreen Supermix (Biorad) and $0.2 \mathrm{mM}$ RT-primers each. The realtime RT-PCR was performed with the IQ5 Biorad machine. All measurements were performed in triplicates and normalized to the values of ornithine decarboxylase (ODC). Two independent biological replicates were performed. The copy numbers were based on a standard dilution series.

\subsubsection{Quantitative Nanostring}

In addition for quantitative measurements, the digital multiplexed gene expression analysis system Nanostring was used according to the manufacturer's instructions using $500 \mathrm{ng}$ of total RNA. The genes analysed by Nanostring as well as the target region and sequences of the reporter probes are shown in Appendix 6.3. To process the data, the counts were first normalized with respect to the geometric mean of the positive control counts using the nSolver software program provided by Nanostring. In a second step, the counts were normalized with respect to the geometric mean of ornithine decarboxylase (ODC). Even though additional housekeeping genes were present in the Nanostring set, only ODC was chosen for normalization as the other genes show developmental regulatory effects. Finally, a stringent background correction was performed by subtracting the mean and two standard deviations of the eight negative control counts for each lane. Negative 
values were set to 1 as background level. Two independent experiments were performed.

\subsubsection{RNA-sequencing}

\subsection{RNA isolation}

For RNA-sequencing, total RNA was isolated with trizol as described above with the following modifications:

The ectodermal explants were lysated in $360 \mu \mathrm{l}$ trizol with a 29-Gauge syringe and incubated for $10 \mathrm{~min}$ at RT. $72 \mu \mathrm{l}$ chloroform was added, mixed and incubated for further $5 \mathrm{~min}$ at RT. After centrifugation for $20 \mathrm{~min}$ at $4{ }^{\circ} \mathrm{C}$, the upper phase, containing the total RNA, was transferred to a new eppendorf tube. $180 \mu \mathrm{l}$ isopropanol was added, mixed well and incubated at $-20{ }^{\circ} \mathrm{C}$ for at least $2 \mathrm{~h}$. After centrifugation at $4{ }^{\circ} \mathrm{C}$ for $30 \mathrm{~min}$, the precipitate was washed with $500 \mu \mathrm{l}$ of $75 \%$ ethanol. The pellet was air-dried and dissolved in $20 \mu \mathrm{l}$ of RNase-free water. For genomic DNA digestion, the RNA was incubated for $1 \mathrm{~h}$ at $37{ }^{\circ} \mathrm{C}$ in a $50 \mu \mathrm{l}$ reaction containing $1 \mathrm{x}$ DNasel reaction buffer (Thermo Scientific), $1 \mu \mathrm{l}$ DNasel (Thermo Scientific) and $0.5 \mu$ RNAse inhibitor (Thermo Scientific). The DNasel digest was stopped by the addition of $150 \mu$ RNase-free water and $200 \mu \mathrm{l}$ Phenol-Chloroform-Isoamylalcohol. After mixing well, the solution was centrifuged for $10 \mathrm{~min}$ at $4{ }^{\circ} \mathrm{C}$ and the upper phase transferred to a new eppendorf tube. To the upper phase, 1/10 vol. of $5 \mathrm{M}$ ammonium acetate and $1 \mathrm{vol}$ of isopropanol was added and incubated for at least $2 \mathrm{~h}$ at $-20{ }^{\circ} \mathrm{C}$. After centrifugation for $30 \mathrm{~min}$ at $4{ }^{\circ} \mathrm{C}$, the pellet was washed twice with $75 \%$ ethanol and air-dried. The pellet was resolved in $25 \mu$ l RNase-free water.

To evaluate the RNA quality, an analysis with the 2100 Bioanalyzer (Agilent) was performed. To check for genomic DNA contamination a control PCR with histone $\mathrm{H} 4$ was performed and analysed on a $2 \%$ agarose gel.

\subsection{Sample preparation and sequencing}

For sequencing, the RNA-samples were prepared with the "TruSeq RNA Sample Prep Kit v2" according to the manufacturer (Illumina). The sequencing was performed using the HiSeq 2000 (Illumina). Two independent biological replicates were performed. 


\subsection{Sequencing alignment}

After quality control using FastQC (http://h.bioinformatics.babraham.ac.uk/projects/fastqc/), the sequence reads consisting of $2 \times 95$ base pairs from the paired-end mode were first aligned to the Xenopus tropicalis (X. tropicalis) using JGI v72b (kindly provided by Michael J. Gilchrist). Additionally, the reads were also aligned to selected sequences of the $\mathrm{X}$. laevis transcriptome (UniGene Build \#91 X. laevis) representing genes which were not found in the $X$. tropicalis transcriptome to get more information and to avoid redundancies.

The mapping was performed using the software Bowtie2 (version 2.0.0-beta5) in local alignment mode allowing 3 mismatches (Langmead and Salzberg, 2012). Reads aligned greater than 2 times were removed to facilitate the analysis.

\subsection{Statistical analysis}

First, the reads were counted on transcript level and the gene level expression was calculated as the sum of counts from all associated transcripts. After normalization between library samples, gene models with less than 50 read counts were subtracted from the data set. For the detection of differentially expressed genes, the DESeq package (Anders and Huber, 2010) (version 1.4.1) of bioconductor (http://h.bioconductor.org) was used.

\subsubsection{Xenopus laevis embryo culture and microinjections}

\subsubsection{Stimulation of eggs}

$X$. laevis female frogs were induced to lay eggs by the injection of 1000 units human chorionic gonadotropin (HCG, Sigma Aldrich) into the dorsal lymph sac the evening before the supposed egg-laying. The frogs were kept at $16{ }^{\circ} \mathrm{C}$ overnight. To induce the egg-laying later in the afternoon, the female $X$. laevis frogs were injected with 500 units of HCG into the dorsal lymph sac the evening before and kept at RT over night. The next morning the frogs were injected with 1000 units of HCG. 


\subsubsection{Preparation of Xenopus laevis testis}

10x MBS: $880 \mathrm{mM} \mathrm{NaCl}, 10 \mathrm{mM} \mathrm{KCl}, 10 \mathrm{mM} \mathrm{MgSO}_{4}, 25 \mathrm{mM} \mathrm{NaHCO} 3,50 \mathrm{mM}$ HEPES, pH 7.8

1x MBS: 1x MBS, $0.7 \mathrm{mM} \mathrm{CaCl} 2$

For in vitro fertilization of the eggs, the male $X$. laevis frog was sacrificed and the testis was removed, washed in $1 \mathrm{x} \mathrm{MBS}$ and stored at $4{ }^{\circ} \mathrm{C}$. For the fertilization, around one third of the testis was cut and diluted in 1x MBS and stored on ice.

\subsubsection{Fertilization}

Cysteine: $2 \%$ L-cysteine hydrochloride in 0.1x MBS, pH 8.0

10x MBS: $880 \mathrm{mM} \mathrm{NaCl}, 10 \mathrm{mM} \mathrm{KCl}, 10 \mathrm{mM} \mathrm{MgSO}_{4}, 25 \mathrm{mM} \mathrm{NaHCO}_{3}, 50 \mathrm{mM}$ HEPES, $\mathrm{pH} 7.8$

\section{Nile Blue: $0.01 \%(w / v)$ Nile blue in $0.1 \times$ MBS}

The layed eggs were in vitro fertilized using $50 \mu$ of the testis diluted with $450 \mu$ l water. To remove the jelly coat, the eggs were treated with $2 \%$ cysteine hydrochloride, $\mathrm{pH} 8.0$ and washed three times with 0.1x MBS. Albino embryos were additionally stained with Nile Blue vital dye for 5 min and washed three times with $0.1 \times \mathrm{MBS}$.

\subsubsection{Microinjections}

Injection buffer: $2 \%$ Ficoll in 1x MBS

Microinjections were performed on a cooling plate at $12.5{ }^{\circ} \mathrm{C}$. For microinjections, the mRNA was loaded into glass needles, which were prepared on a needle puller (PN-30, Science Products $\mathrm{GmbH}$, Hofheim). The microinjector PV 820 (H. Sauer, Reutlingen) was used. During and after the injections, the embryos were kept in injection buffer. $4 \mathrm{nl}$ of the mRNA were injected either in one or in both blastomeres of two-cell stage embryos. After $1 \mathrm{~h}$ incubation in injection buffer, the embryos were transferred into 0.1x MBS and cultured in incubators until the desired developmental stage was reached. The 
developmental stage of the embryos was defined according to Nieuwkoop and Faber.

\subsubsection{Xenopus laevis ectodermal explants ("animal caps")}

5xMBS AC: $880 \mathrm{mM} \mathrm{NaCl}, 10 \mathrm{mM} \mathrm{KCl}, 10 \mathrm{mM} \mathrm{MgSO}_{4}, 25 \mathrm{mM} \mathrm{NaHCO}_{3}, 2.05$ $\mathrm{mM} \mathrm{CaCl}_{2}, 1.65 \mathrm{mM} \mathrm{Ca}\left(\mathrm{NO}_{3}\right)_{2}, \mathrm{pH} 7.8$

Animal caps were cut at blastula stage (stage 8 to 9 ) on a $1 \%$ agarosecoated Petri-dish in $0.8 \times \mathrm{MBS}$ on a cooling plate set at $12.5^{\circ} \mathrm{C}$ (Wallingford and Harland 2001). First, the vitelline membrane was carefully removed with foreceps and then the animal caps were excised from the animal hemisphere of the embryo using the gastromaster system (Xenotek Engineering, Bellville, USA). The ectodermal explants were cultivated in 0.8x MBS until control whole embryos reached the desired developmental stage.

\subsubsection{Treatment of whole embryos and animal caps}

\subsection{Dexamethasone (Dex) treatment}

\section{Dexamethasone}

stock: $4 \mathrm{mg} / \mathrm{ml}$ in $100 \%$ ethanol, working: $10 \mu \mathrm{M}$

To control the time point of protein activity, hormone-inducible constructs were used for microinjections. Therefore, the coding regions were fused to the ligand-binding domain of the glucocorticoid receptor (Gammill and Sive, 1997). To induce protein activity, injected embryos or animal caps were cultivated in the dark in the presence of $10 \mu \mathrm{M}$ dexamethasone in 0.8x MBS until the desired stage was reached (Perron et al., 1999). For the 3 and $6 \mathrm{~h}$ time point, the animal caps were incubated at $14{ }^{\circ} \mathrm{C}$. For the $9 \mathrm{~h}$ and the $25 \mathrm{~h}$ as well as for the $32 \mathrm{~h}$ time point, the animal caps were incubated at $16{ }^{\circ} \mathrm{C}$ and $18{ }^{\circ} \mathrm{C}$, respectively.

\subsection{Cycloheximide (CHX) treatment}

\section{Cycloheximide}

stock: $100 \mathrm{mg} / \mu \mathrm{l}$ in DMSO (Sigma-Aldrich), working: $10 \mu \mathrm{g} / \mathrm{ml}$ 
To block protein synthesis, animal caps were treated with $10 \mu \mathrm{g} / \mathrm{ml} \mathrm{CHX}$ in $0.8 \mathrm{x}$ MBS (Perron et al., 1999) and incubated at $16{ }^{\circ} \mathrm{C}$.

\subsubsection{Whole mount in situ hybridization}

Whole mount in situ hybridization was performed to visualize the spatial and temporally expression of endogenous RNA transcripts. For detection, a Digoxigenin or Fluorescin labeled antisense RNA probe was used, which was visualized using an alkaline phosphatase-coupled anti-Dig antibody or FastRed (Roche) (Harland, 1991; Hollemann and Pieler, 1999; Nieber et al., 2009).

\subsubsection{Fixation and $\beta-G a l$ staining}

10x MEM: 1 M Mops, 20 mM EGTA, $10 \mathrm{mM} \mathrm{MgSO}_{4}$, pH 7.4, sterile filtered and stored in dark

10x PBS: $1.75 \mathrm{M} \mathrm{NaCl}, 1 \mathrm{M} \mathrm{KCl}, 65 \mathrm{mM} \mathrm{Na}_{2} \mathrm{HPO}_{4}, 18 \mathrm{mM} \mathrm{KH}_{2} \mathrm{PO}_{4}$, pH 7.4

$\mathrm{K}_{3} \mathrm{FE}(\mathrm{CN})_{6}: 0.5 \mathrm{M}$ in $\mathrm{H}_{2} \mathrm{O}$, stored in dark

$\mathrm{K}_{4} \mathrm{FE}(\mathrm{CN})_{6}$ : $0.5 \mathrm{M}$ in $\mathrm{H}_{2} \mathrm{O}$ stored in dark

MEMFA: 4\% (v/v) formaldehyde (37\%) in 1x MEM

X-Gal: $\quad 40 \mathrm{mg} / \mathrm{ml}$ 5-Bromo-4-chloro-3-indolyl-b-D-galactopyranoside in formamide, stored $-20^{\circ} \mathrm{C}$ in the dark

X-Gal staining solution: $1 \mathrm{mg} / \mathrm{ml} X-G a l, 5 \mathrm{mM} \mathrm{K}_{3} \mathrm{FE}(\mathrm{CN})_{6}, 5 \mathrm{mM} \mathrm{K}_{4} \mathrm{FE}(\mathrm{CN})_{6}, 2$ $\mathrm{mM} \mathrm{MgCl} 2$ in $1 \times$ PBS

Embryos were collected in glas vials and fixed in MEMFA at RT. Uninjected-embryos were fixed for $1 \mathrm{~h}$ and washed three times with $100 \%$ ethanol for $5 \mathrm{~min}$. Injected embryos were fixed for $25 \mathrm{~min}$ and washed three times with 1x PBS for 10 min.

LacZ ( $\beta$-gal) mRNA was co-injected as a lineage tracer to discriminate the injected from the uninjected side of the embryo and to analyse the distribution of the injected mRNA. To visualize the $\beta$-gal injected side of the embryos, X-Gal staining was performed (Hardcastle et al., 2000). Therefore, the embryos were incubated in the dark in X-gal solution for at least 20 min until the desired level of staining was achieved. Afterwards, the embryos were washed three times for 
$10 \mathrm{~min}$ in 1x PBS and refixed for $25 \mathrm{~min}$ in 1x MEMFA. Finally, the embryos were washed three times for 5 min in $100 \%$ ethanol and stored at $-20{ }^{\circ} \mathrm{C}$.

\subsubsection{Rehydration}

PTW: $0.1 \%$ Tween-20 in 1x PBS

The following steps were performed at RT in $4 \mathrm{ml}$ solution with mild agitation. The embryos were rehydrated using an ethanol series $(75 \%, 50 \%$, $25 \%$ ) and finally incubated 4 times for 5 min in 100\% PTW.

\subsubsection{Proteinase $\mathrm{K}$ treatment}

Proteinase K: $10 \mu \mathrm{g} / \mathrm{ml}$ proteinase $\mathrm{K}$ in $0.1 \times$ PBS

PTW: $0.1 \%$ Tween-20 in $1 \times$ PBS

To make the embryos accesible to the Dioxigenin labeled antisense RNA probe, the embryos were treated with proteinase $\mathrm{K}$ (Merck). Therefore, the embryos were incubated in a $2 \mathrm{ml}$ PTW/proteinase K-solution (10 $\mu \mathrm{g} / \mathrm{ml} \mathrm{PTW}$ ) for a defined time depending on the developmental stage. Embryos of developmental stage 28/29 were treated for $17 \mathrm{~min}$ in proteinase K solution.

\subsubsection{Acetylation and refixation}

PTW: $0.1 \%$ Tween-20 in 1x PBS

0.1 M Triethanolamine: $0.93 \mathrm{~g}$ Triethanolamine in $\mathrm{H}_{2} \mathrm{O}, \mathrm{pH} 7.5$

To stop the proteinase $\mathrm{K}$ treatment, the embryos were washed two times for $5 \mathrm{~min}$ in triethanolamine. To avoid unspecific reaction of the Dioxigenin labeled antisense RNA probe, free amino-acid ends were blocked by treating the embryos with $25 \mu \mathrm{l}$ acetic anhydride in triethanolamine. Afterwards the embryos were washed two times for 5 min with PTW and refixed in $4 \%$ formaldehyde (v/v) in PTW for $20 \mathrm{~min}$. Before hybridization, the embryos were washed again 5 times for 5 min in PTW. 


\subsubsection{Hybridization}

Hybridization Mix: 50\% formamide, $1 \mathrm{mg} / \mathrm{ml}$ Torula RNA, $10 \mu \mathrm{g} / \mathrm{ml}$ Heparin, $1 \mathrm{x}$ Denhardt's, 0.1\% Tween-20, 0.1\% CHAPS, $10 \mathrm{mM}$ EDTA in 5x SSC 20X SSC: $3 \mathrm{M} \mathrm{NaCl}, 0.3 \mathrm{M} \mathrm{NaCitrate,} \mathrm{pH}$ 7.2-7.4 100x Denhardts solution: $1 \mathrm{~g}$ BSA, $1 \mathrm{~g}$ Polyvinylpyrollidone (PVP), $1 \mathrm{~g}$ Ficoll,

For pre-hybridization, the embryos were first incubated in $1 \mathrm{ml}$ fresh hybridization mix and then after exchanging the hybridization mix, for at least 5 $\mathrm{h}$ at $65{ }^{\circ} \mathrm{C}$. Afterwards, the embryos were incubated in $1 \mathrm{ml}$ hybridization mix containing the Dioxigenin labeled antisense RNA probe overnight at $65^{\circ} \mathrm{C}$.

\subsubsection{Washing}

20X SSC: $3 \mathrm{M} \mathrm{NaCl}, 0.3 \mathrm{M} \mathrm{NaCitrate,} \mathrm{pH}$ 7.2-7.4

MAB: $100 \mathrm{mM}$ maleic acid, $150 \mathrm{mM} \mathrm{NaCl}, \mathrm{pH} 7.5$

The next morning, the hybridization mix conatining the antisense RNA probe was exchanged for fresh hybridization mix for $10 \mathrm{~min}$ at $65{ }^{\circ} \mathrm{C}$. Afterwards, the embryos were washed three times with 2x SSC for 15 min at 65 ${ }^{\circ} \mathrm{C}$. To remove unspecific and unbound RNA probes, the embryos were washed and treated for $1 \mathrm{~h}$ at $37^{\circ} \mathrm{C}$ with RNAse $\mathrm{A}(10 \mu \mathrm{g} / \mathrm{ml}$, Fermentas) and RNAse T1 (10 U/ml, Fermentas) in 2x SSC. After a short washing step with 2x SCC for $5 \mathrm{~min}$, the embryos were incubated two times in $0.2 x \mathrm{SSC}$ at $65^{\circ} \mathrm{C}$. Finally, the embryos were transferred into MAB and incubated two times for $15 \mathrm{~min}$.

\subsubsection{Blocking and antibody reaction}

MAB: $100 \mathrm{mM}$ maleic acid, $150 \mathrm{mM} \mathrm{NaCl}, \mathrm{pH} 7.5$

MAB/BMB: $2 \%$ BMB in 1x MAB

MAB/BMB/HS: 2\% BMB, 20\% heat-treated horse serum in 1x MAB

To avoid additionally unspecific binding, the embryos were treated with blocking buffer containing MAB and the Boehringer Mannheim Blocking reagent (BMB) as well as horse serum (Gibco). First, the embryos were washed 15 min in $M A B / B M B$, which was exchanged for $M A B / B M B / H S$ for 40 min. For detection, 
the Sheep alkaline phosphatase-coupled anti-Dig antibody (1:5000, Sigma) was used and the embryos were incubated for $4 \mathrm{~h}$ at RT. Afterwards, the embryos were washed 3 times for 10 min and then overnight at $4{ }^{\circ} \mathrm{C}$ with MAB.

\subsubsection{Staining reaction}

APB: $100 \mathrm{mM}$ Tris- $\mathrm{HCl}, \mathrm{pH}$ 9.0, $50 \mathrm{mM} \mathrm{MgCl}_{2}, 100 \mathrm{mM} \mathrm{NaCl}, 0.1 \%$ Tween-20, $\mathrm{pH} 9.0$

NBT: $100 \mathrm{mg} / \mathrm{ml}$ in 70\% Dimethylformamide, stored in dark

BCIP: $50 \mathrm{mg} / \mathrm{ml}$ in 100\% Dimethylformamide, stored in dark

The next day, the embryos were washed 5 times for 5 min with MAB, followed by two times for 5 min with $\mathrm{ABP}$ at $4{ }^{\circ} \mathrm{C}$. The staining reaction was performed in the dark until the desired staining was reached using a mixture of BCIP/NBT, a substrate of the alkaline phosphatase.

\subsubsection{Destaining and refixation}

To remove background staining, the embryos were treated stepwise with methanol of different perncentage $(100 \%, 75 \%, 50 \%, 25 \%)$. Finally, the embryos were refixed with MEMFA for $15 \mathrm{~min}$ at RT. The MEMFA was replaced by fresh MEMFA for long storage of the embryos at $4{ }^{\circ} \mathrm{C}$.

\subsubsection{Bleaching}

20X SSC: $3 \mathrm{M} \mathrm{NaCl}, 0.3 \mathrm{M} \mathrm{NaCitrate,} \mathrm{pH}$ 7.2-7.4

MEMFA: $4 \%(v / v)$ formaldehyde (37\%) in 1x MEM

Pigmented embryos were bleached using one of two different methods and afterwards fixed with MEMFA for long storage.

To reduce the background, the embryos were first destained with $100 \%$ methanol. For a more sensitive bleaching, the embryos were incubated in a mixture of $75 \%$ methanol $/ \mathrm{H}_{2} \mathrm{O}_{2}$ for up to three days, followed by treatments with $50 \%$ and $25 \%$ methanol. More time efficient, the embryos were incubated for 1 to $2 \mathrm{~h}$ in the following solution at RT: $5 x$ SSC, $50 \%$ formamide and $1-2 \% \mathrm{H}_{2} \mathrm{O}_{2}$. Finally, two washing steps with $5 \mathrm{x}$ SSC for 5 min were performed before fixation. 


\subsubsection{Vibratome sectioning}

Gelatin/Albumin: $4.88 \mathrm{mg} / \mathrm{ml}$ gelatin was dissolved in PBS. Afterwards, 0.3 $\mathrm{g} / \mathrm{ml}$ bovine serum albumin and $0.2 \mathrm{mg} / \mathrm{ml}$ sucrose were added and dissolved. The solution was sterile filtered and stored at $-20^{\circ} \mathrm{C}$.

Mowiol: $5 \mathrm{~g}$ Mowiol was dissolved in $20 \mathrm{ml}$ PBS by stirring for more than $16 \mathrm{~h}$. Afterwards, $10 \mathrm{ml}$ glycerol was added and stirred for $16 \mathrm{~h}$. The supernatant was recovered and a $\mathrm{pH}$ of 7.0 was adjusted. The solution was stored at $-20{ }^{\circ} \mathrm{C}$.

For vibratome sectioning, the whole embryos were embedded in a mixture of gelatin and albumin by adding gluturylaldehyde. The embryos were vibratome sectioned at $30 \mu \mathrm{M}$ using a vibratome (Leica VT1000M). The sections were collected on glass slides and covered with a cover slip using mowiol.

\subsubsection{Protein standard techniques}

\subsubsection{Protein-protein interaction in vivo (Co- immunoprecipitation)}

Table 2.4. Summary of antibodies and working dilutions

\begin{tabular}{|l|l|l|l|l|}
\hline & \multicolumn{1}{|c|}{ Name } & Company & dilution WB & dilution IP \\
\hline Primary antibody & Mouse monoclonal HA.11 & Covance & $1: 1000$ & $1: 150$ \\
\hline & Goat polyclonal MT & Abcam & $1: 10000$ & $1: 250$ \\
\hline Secondary antibody & anti-mouse-HRP & Santa Cruz & $1: 5000$ & \\
\hline & anti-goat-HRP & Santa Cruz & $1: 10000$ & \\
\hline
\end{tabular}

Co-IP buffer: $10 \mathrm{mM}$ Tris-HCl, pH 7.5, $100 \mathrm{mM} \mathrm{NaCl}, 2 \mathrm{mM}$ EDTA, $1 \mathrm{mM}$ EGTA, $0.5 \%$ (v/v) NP-40, $10 \%(\mathrm{v} / \mathrm{v})$ glycerol, $1 \mathrm{mM}$ Sodium-Orthovanadate, $1 \mathrm{mM}$ b-Glycerolphosphate, $1 \mathrm{mM} \mathrm{NaF}$, protease inhibitor cocktail (1 tablet per $50 \mathrm{ml}$ Co-IP buffer, Roche)

6x SDS sample buffer: $125 \mathrm{mM}$ Tris $\mathrm{pH}$ 6.8, 30\% glycerol, 10\% SDS, $0.6 \mathrm{M}$ DTT, $0.012 \%$ Bromphenol blue

For a Co-immunoprecipitation assay, 25 injected embryos were harvested at gastrula stage. The embryos were lysated with a 27-gauge-syringe in $500 \mu \mathrm{l} \mathrm{Co-IP}$ buffer. The lysates were centrifuged for $15 \mathrm{~min}$ at $4{ }^{\circ} \mathrm{C}$ and the 
supernatant was transferred to a new eppendorf tube. $50 \mu$ l of the lysates were transferred to a new eppendorf tube and served later as input control (10\%). To the input samples, $10 \mu \mathrm{l} 6 \mathrm{x}$ SDS loading buffer was added and heated for $3 \mathrm{~min}$ at $95{ }^{\circ} \mathrm{C}$ before storing at $-20{ }^{\circ} \mathrm{C}$. The Co-IP samples were adjusted to $500 \mu \mathrm{l}$ with the Co-IP buffer. For pre-clearing, $30 \mu \mathrm{l}$ of $\mathrm{Y}$-bind sepharose immunopellets (GE Healthcare) were added. The Co-IP samples were incubated for $30 \mathrm{~min}$ at $4{ }^{\circ} \mathrm{C}$ on a rotating wheel and then centrifuged for $20 \mathrm{sec}$ at $2,000 \mathrm{rpm}$. Afterwards, the supernatants were transferred to a new eppendorf tube and split in $250 \mu \mathrm{l}$ each. To the Co-IP samples the specific antibodies were added. The samples were incubated for $2 \mathrm{~h}$ at $4{ }^{\circ} \mathrm{C}$ on a rotating wheel. Afterwards $20 \mu \mathrm{l}$ of the Y-bind sepharose immunopellets was added. The Co-IP samples were incubated for another $2 \mathrm{~h}$ at $4{ }^{\circ} \mathrm{C}$ on a rotating wheel. Finally, the samples were centrifuged for $30 \mathrm{sec}$ at 2,000 rpm and the pellets were washed 5 times with 1 $\mathrm{ml}$ Co-IP buffer. The supernatant was removed and $10 \mu \mathrm{l}$ of $6 \mathrm{x}$ loading buffer were added. The Co-IP samples were incubated for $3 \mathrm{~min}$ at $95^{\circ} \mathrm{C}$ and stored at $-20{ }^{\circ} \mathrm{C}$.

\subsubsection{SDS-Polyacrylamide gel electrophoresis}

10x Laemmli buffer: $250 \mathrm{mM}$ Tris-base, $2.5 \mathrm{M}$ Glycine, $0.1 \%$ SDS

To separate denatured proteins according to their molecular weight in an electric field, SDS-polyacrylamide gel electrophoresis was performed (Laemmli, 1970). To denaturate the proteins, the samples were diluted with $6 x$ SDS loading buffer and boiled for $3 \mathrm{~min}$ at $95^{\circ} \mathrm{C}$ in a water bath. For separation of the denatured proteins, a $12 \%$ SDS gel was used and prepared according to standard protocols (Sambrook and Russel, 2001). To visualize the size of the

proteins molecular weight, a standard protein marker was used (Page Ruler ${ }^{\mathrm{TM}}$ prestained protein ladder, Thermo Scientific). The gel electrophoresis was performed using $1 \mathrm{x}$ Laemmli buffer as running buffer. First, a voltage of $100 \mathrm{~V}$ was applied and when the bromphenol-blue front reached the separating gel, the voltage was raised to $250 \mathrm{~V}$.

\subsubsection{Western blot}

Blocking Buffer: $5 \%(w / v)$ dry, non fat-milk powder in PBS-T 
PBS-T: 1x PBS, 0.5\% (v/v) Tween 20

Anode blotting buffer 1: $0.3 \mathrm{M}$ Tris, $10 \%$ methanol, $\mathrm{pH} 10.4$

Anode blotting buffer 2: $25 \mathrm{mM}$ Tris, 10\% methanol, $\mathrm{pH} 10.4$

Cathode blotting buffer: $25 \mathrm{mM}$ Tris, $40 \mathrm{mM}$ glycine, 10\% methanol, pH 9.4

After separation, the proteins were transferred from the polyacrylamide gel to a nitrocellulose membrane using the semi-dried blotting method for $1 \mathrm{~h}$ (Sambrook and Russel, 2001; Towbin et al., 1979). To minimize unspecific binding, the nitrocellulose membrane was blocked for $1 \mathrm{~h}$ at RT using $5 \%(\mathrm{w} / \mathrm{v})$ milk powder in PBS-T buffer. Afterwards, the nitrocellulose membrane was incubated overnight at $4{ }^{\circ} \mathrm{C}$ in milk buffer containing the first antibody. The following morning, the membrane was washed with $1 \times$ PBS-T for 30 min at RT thereby exchanging the buffer every ten minutes. Afterwards, the second antibody in milk buffer was added. After $1 \mathrm{~h}$ incubation at RT, the nitrocellulose membrane was again washed for 30 min with 1x PBS-T. For detection, the membrane was incubated with the chemiluminescent substrate "Super Signal West Dura" (Thermo Scientific) according to the manufacturer's protocol and then exposed to X-ray detection films (Amersham). 


\section{Results}

\subsection{Ptf1a promotes general neurogenesis and specifies GABAergic inhibitory interneurons at the expense of other neuronal cell types}

The bHLH transcription factor Ptf1a functions in the nervous system to promote generation of GABAergic inhibitory interneurons at the expense of glutamatergic excitatory neurons in the spinal cord, cerebellum and retina (Glasgow et al., 2005; Hoshino et al., 2005; Fujitani et al., 2006; Nakhai et al., 2007; Pascual et al., 2007; Dullin et al., 2007). Microinjection of Ptf1a-GR mRNA is sufficient to induce ectopic neurons in animal caps and in the nonneural ectoderm of $X$. laevis embryos and these neurons have a GABAergic identity (Dullin et al., 2007; Hedderich, 2008). Thus, Ptf1a not only acts as a GABAergic selector gene in the post-mitotic neurons, but also drives neuronal cell commitment of progenitor cells.

In the mouse, Ptf1a is expressed in the dorsal horn of the spinal cord in the progenitor domain of the $\mathrm{dl} 4$ and later $\mathrm{dll}_{\mathrm{A}}$ neurons, which give rise to GABAergic inhibitory interneurons (Glasgow et al., 2005); the dl4 and $\mathrm{dll}_{\mathrm{A}}$ neurons can be molecularly identified by a specific code of HD transcription factors consisting of Pax2, Lbx1, Lhx1 and Lhx5 (Glasgow et al., 2005; Helms and Johnson, 2003). In X. laevis, the domains and combinatorial codes of transcription factors that characterise distinct neuronal subtype domains are not well defined. Therefore, to evaluate Ptf1a activity during the development of the $X$. laevis nervous system, gain-of-function experiments analysing marker genes indicative of specific neuronal subtype identities in embryos were performed. To control the onset of protein activity, the ligand-binding domain of the glucocorticoid receptor was fused to the C-terminus of Ptf1a (Ptf1a-GR) (Afelik et al., 2006). In the absence of the ligand dexamethasone (Dex), the fusion proteins are bound by heat shock proteins and remain transcriptionally inactive in the cytoplasm. Upon addition of dexamethasone, the fusion proteins translocate into the nucleus and regulate transcription of target genes (Gammill and Sive, 1997).

Ptf1a-GR mRNA was injected into one blastomere of two-cell stage embryos together with LacZ mRNA, in order to localize the distribution of the 
injected mRNA. At early gastrula stage (stage 10), the injected embryos were treated with dexamethasone to induce Ptf1a-GR protein activity. In agreement with previous reports (Dullin et al., 2007; Hedderich, 2008), overexpression of Ptf1a-GR mRNA in the $X$. laevis hindbrain and spinal cord promoted a dramatic increase in expression of the post-mitotic neuronal marker $N$-tubulin and of Glutamic acid decarboxylase (Gad1), an enzyme that catalyses the synthesis of GABA and is indicative of GABAergic inhibitory neurons (Li et al., 2006) (Fig. 3.1 B, B' and C, C'). In line with studies in the murine nervous system (Glasgow et al., 2005), overexpression of Ptf1a-GR mRNA also promoted the activation of Pax2 and $L h x 1$, which mark dorsal interneuron populations (Fig. 3.1 E, E' and F, $\left.F^{\prime}\right)$. Additionally, $L b x 1$ expression was induced, which also encodes for a transcription factor required in the post-mitotic neurons to specify a GABAergic interneuronal subtype (Fig. 3.1 D, D') (Cheng et al., 2005). In contrast, transcription factors expressed in neuronal domains more ventral to the endogenous Ptf1a progenitor domain, such as Hox11L2 or Lmx1b (Fig. $3.1 \mathrm{G}$, $\mathrm{G}^{\prime}$ and $\mathrm{H}, \mathrm{H}$ ) were inhibited by Ptf1a. Similar results were reported for Ptf1a in the murine spinal cord (Glasgow et al., 2005).

While Ptf1a is thought to function as a transcriptional activator (Cockell et al., 1989; Krapp et al., 1996), many transcription factors have been shown to act both as transcriptional activators or repressors in a context-dependent fashion (lype et al., 2004; Yu et al., 2005; Sakabe et al., 2012). The expression of the above marker genes was therefore evaluated upon microinjection of a hormone-inducible version of Ptf1a that was fused to a heterologous strong activator domain derived from VP16 (Ptf1a-VP16-GR) (Sadowski et al., 1988; Dullin et al., 2007). Similar to Ptf1a-GR, overexpression of Ptf1a-VP16-GR mRNA induced strong expression of the GABAergic interneuronal markers Gad1, Pax2, Lhx1 and Lbx1 (Fig. 3.1 J, J'; K, K'; L, L' and M, M') as well as of the general neuronal marker $N$-tubulin (Fig. 3.1 I, l'). Moreover, the expression of Hox11L2 and Lmx1b was still inhibited (Fig. 3.1 N, N' and O, O'), suggesting that the repression by Ptf1a is indirect.

The ability of Ptf1a to induce general neurogenesis in the non-neural ectoderm of $X$. laevis embryos and in animal caps is similar to that of the proneural bHLH transcription factor Neurogenin 2 (Ngn2, also known as X-ngnr1) (Ma et al., 1996). However, while Ptf1a induces Gad1, a GABAergic marker 
in such assays (Dullin et al., 2007; Hedderich, 2008), Ngn2 promotes the expression of Hox11L2, a marker for cranial sensory ganglia and Rohon-Beard sensory neurons (Perron et al., 1999; Dullin et al., 2007; Hedderich, 2008). Hox11L2 is the $X$. laevis ortholog of $T / x 3$, which has been shown in mouse to act as a selector gene promoting a glutamatergic excitatory at the expense of a GABAergic inhibitory cell fate (Cheng et al., 2004; Cheng et al., 2005). In the $X$. laevis neural tube, overexpression of Ngn2-GR mRNA strongly activated the expression of $N$-tubulin (Fig. 3.1 P, $\mathrm{P}^{\prime}$ ), similar to Ptf1a-GR and Ptf1a-VP16-GR mRNA. In contrast, Ngn2-GR mRNA did not influence Gad1, Pax2, Lbx1 or Lhx1 expression (Fig. 3.1 Q, Q'; R, R'; S, S'; T, T'), but instead led to ectopic activation of Hox11L2/TIx3 (Fig. 3.1 V, V'). Moreover, Ngn2-GR mRNA had no effect on the expression of $L m \times 1 b$ (Fig. $3.1 \mathrm{U}, \mathrm{U}^{\prime}$ ), which was repressed by injection of Ptf1a-GR and Ptf1a-VP16-GR mRNA.

Interestingly, overexpression of Ptf1a-GR and Ptf1a-VP16-GR mRNA not only led to an induction or suppression of the described marker genes, but additionally resulted in a strong increase in tissue formation on the injected side of the embryo (Fig. 3.1 B, B' - O, O'). It can be excluded that the extra tissue formation is a general consequence of mRNA overexpression, as the effect was not upon microinjection of Ngn2-GR mRNA (Fig. 3.1 P, $\left.\mathrm{P}^{\prime}-\mathrm{V}, \mathrm{V}^{\prime}\right)$. However, the identity of the extra tissue promoted by Ptf1a-GR and Ptf1a-VP16-GR mRNA overexpression could not be elucidated so far.

Taken together, these findings demonstrate that Ptf1a like Ngn2 promotes general neurogenesis and that Ptf1a confers GABAergic interneuron subtype specification at the expense of other neuronal subtypes. In contrast, Ngn2 induces a glutamatergic excitatory cell fate. 
A Ptf1a-GR $(20 \mathrm{pg})$

Ptf1a-VP16-GR (20 pg)

Ngn2-GR (20 pg)
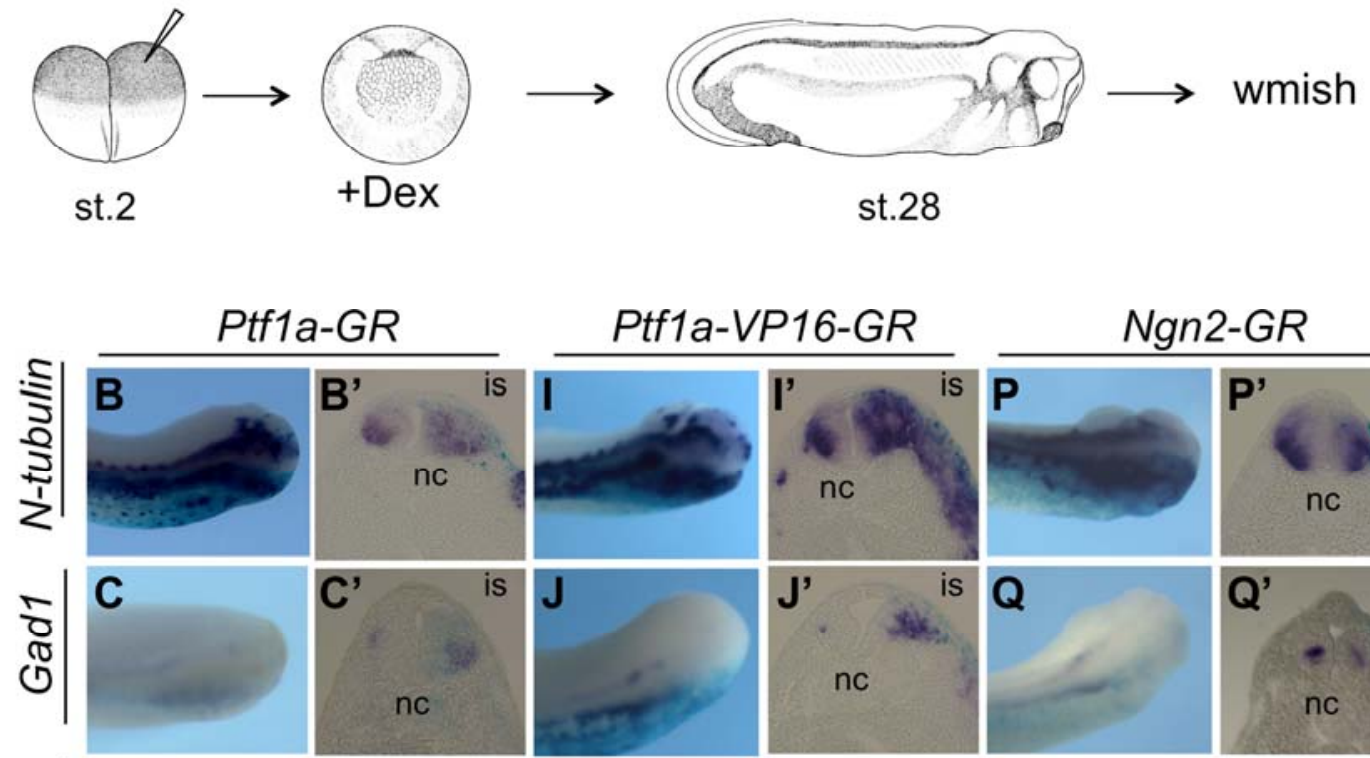

$P 16-G R$

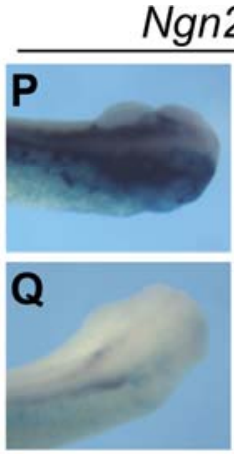

Ngn2-GR
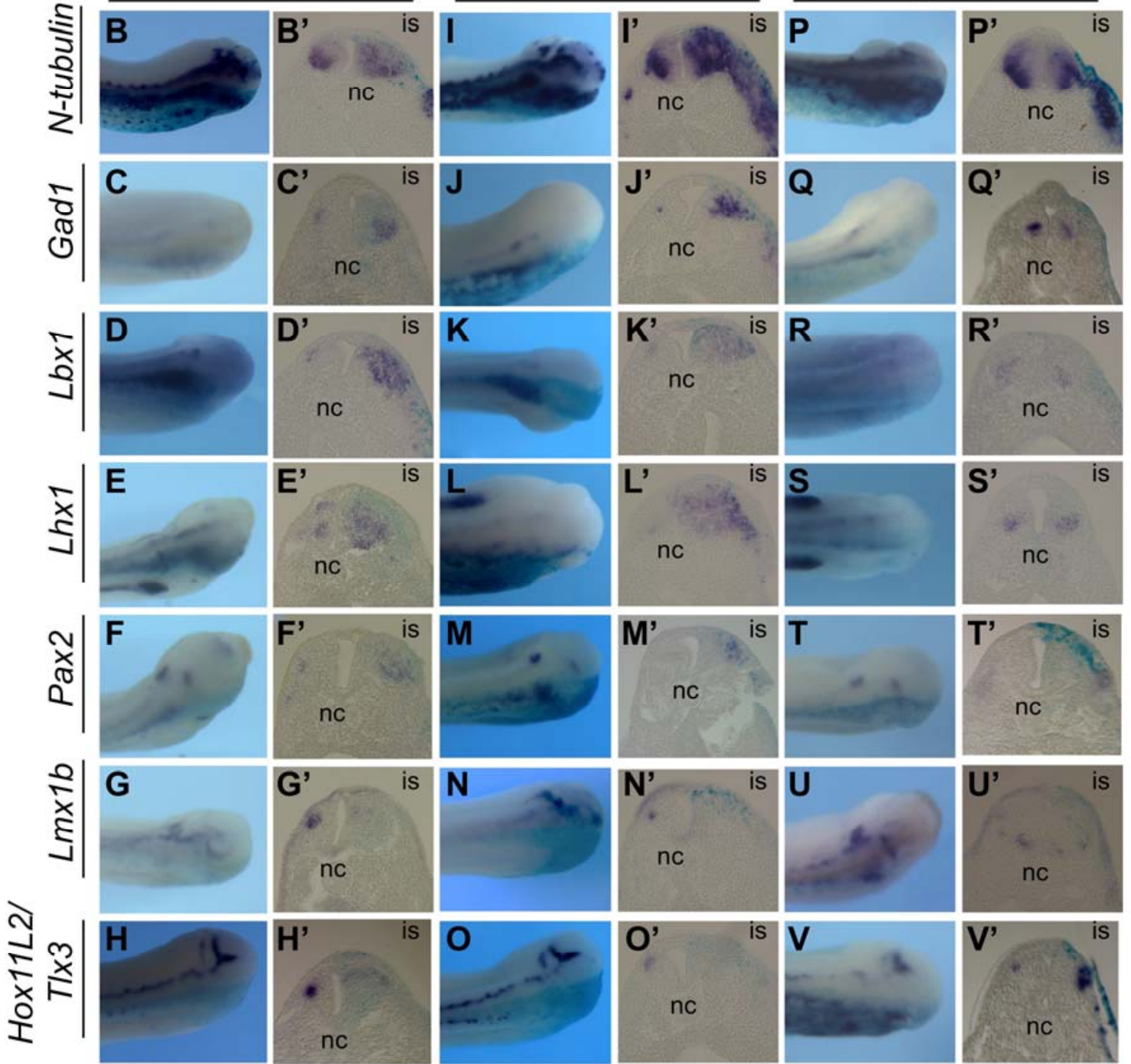

Figure 3.1 Ptf1a promotes general neurogenesis and specifies GABAergic inhibitory interneurons at the expense of other neuronal cell types.

(A) The mRNAs indicated above (20 pg each) were co-injected with LacZ mRNA (75 pg) into one blastomere at the two-cell stage. Protein activity was induced by dexamethasone (Dex) at the equivalent of stage 10. (B-V) Gene expression was analysed by whole mount in situ hybridization (wmish) using markers indicated on the left side. (B-V) Dorsal view of the head region and ( $\left(B^{\prime}-V^{\prime}\right)$ the corresponding transversal section through the hindbrain at the level of the otic vesicle of stage $28 X$. laevis embryos. The injected side is always at the bottom of the whole head view and to the right on the sections. nc, notochord, is, injected side. 


\subsection{Ptf1a and Ngn2 promote general neurogenesis via activation of the same gene network}

The ability of Ptf1a to induce general neuronal differentiation in $X$. laevis embryos is similar to the activity of the bHLH transcription factor Ngn2. However, both transcription factors confer distinct neuronal subtype identities (Fig. 3.1). This raises the question, if Ptf1a and Ngn2 activate the same or a different gene network, to achieve general neurogenesis. Previous results in whole embryos analysing only a limited set of marker genes suggested that Ptf1a and Ngn2 drive general neurogenesis in progenitors through the same cascade of neuronal differentiation factors (Hedderich, 2008). To obtain a higher resolution comparison, a temporal expression analysis of genes induced by Ptf1a-GR and Ngn2-GR mRNA overexpression was performed utilizing the animal cap assay. Animal caps are embryonic pluripotent cells excised from the animal pole of $X$. laevis embryos of the blastula stage. Untreated animal caps will give rise to atypical epidermal tissue. However upon treatment or injection of various factors, the animal caps can obtain cell fates of all three germ layers (Borchers and Pieler, 2010).

Ptf1a-GR or Ngn2-GR mRNA were injected into both blastomeres of twocell stage $X$. laevis embryos, animal caps were excised at blastula stage and treated with dexamethasone to induce protein activity (Fig. 3.2 A). The animal caps were collected 3, 6 and $25 \mathrm{~h}$ after addition of dexamethasone. Total RNA was isolated and the levels of specific mRNAs digitally quantified without amplification using Nanostring nCounter multiplex analysis, which employs target-specific fluorescent barcodes and single molecule imaging (Geiss et al., 2008; Kulkarni, 2011) (Fig. 3.2 and Appendix 6.1.1). The analysis of a selected set of genes is represented in Figure 3.2. Animal caps that were injected, but not treated with dexamethasone served as a negative control and did not significantly express any of the analysed marker genes (Fig. 3.2 B-M). Both Ptf1a-GR and Ngn2-GR mRNA overexpression was sufficient to induce the expression of the Notch-ligand DIl1 (Fig. $3.2 \mathrm{~B}$ ), the cell-cycle inhibitor Cdknx (Fig. 3.2 C) as well as of the zinc-finger transcription factor Myt1 (Fig. 3.2 D) $3 \mathrm{~h}$ after dexamethasone induction. Other neuronal differentiation genes such as Ebf2 (Fig. 3.2 E), Ebf3 (Appendix 6.1.1), NeuroD1 (Fig. 3.2 F) and NeuroD4 (Appendix 6.1.1) were also strongly induced by Ptf1a-GR and Ngn2-GR mRNA 
after $25 \mathrm{~h}$ of protein induction. Similarly, overexpression of Ptf1a-GR and Ngn2GR mRNA led to a high induction of the post-mitotic marker $N$-tubulin (Fig. 3.2 $\mathrm{G}$ ) and the cell cycle regulator Pak3 (Appendix 6.1.1) after $25 \mathrm{~h}$ of protein induction. In regard to GABAergic neuronal subtype specification, Ptf1a-GR but not Ngn2-GR mRNA strongly induced Gad1 (Fig. $3.2 \mathrm{H}$ ) expression as well as the expression of Pax2 (Fig. $3.2 \mathrm{~J}), L h x 5$ (Fig. 3.2 I), $L b x 1$ (Appendix 6.1.1) and Lhx1 (Appendix 6.1.1) after $25 \mathrm{~h}$. In contrast, Ngn2-GR mRNA robustly induced the marker genes for glutamatergic excitatory neurons such as Hox11L2/TIx3 (Fig. 3.2 L) and Vglut1 (Fig. 3.2 M). Furthermore, Ngn2-GR induced the expression of the PR domain containing zinc finger transcription factor Prdm14 more than a 1000-fold (Fig. $3.2 \mathrm{~K}$ ), however, the function of which in the nervous system is still unknown (Fog et al., 2012). Interestingly, Ptf1a-GR mRNA overexpression in animal caps also led to an increase in $L m \times 1 b$ expression (Appendix 6.1.1), a transcription factor involved in glutamatergic excitatory neuron formation and normally suppressed by Ptf1a in the spinal cord (Fig. 3.1 G, G') (Glasgow et al., 2005).

In summary, both Ptf1a and Ngn2 drive early steps of general neurogenesis through activation of an overlapping set of downstream target genes in a similar temporal order. However, later in development, Ptf1a and $\mathrm{Ngn} 2$ induce unique sets of downstream genes to confer specific neuronal subtype identities in post-mitotic neurons. 
A Ptf1a-GR (20 pg)

Ngn2-GR $(20 \mathrm{pg})$

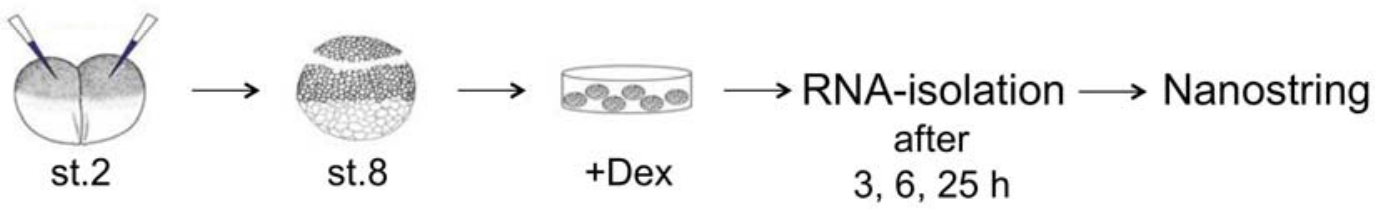

$3,6,25 \mathrm{~h}$
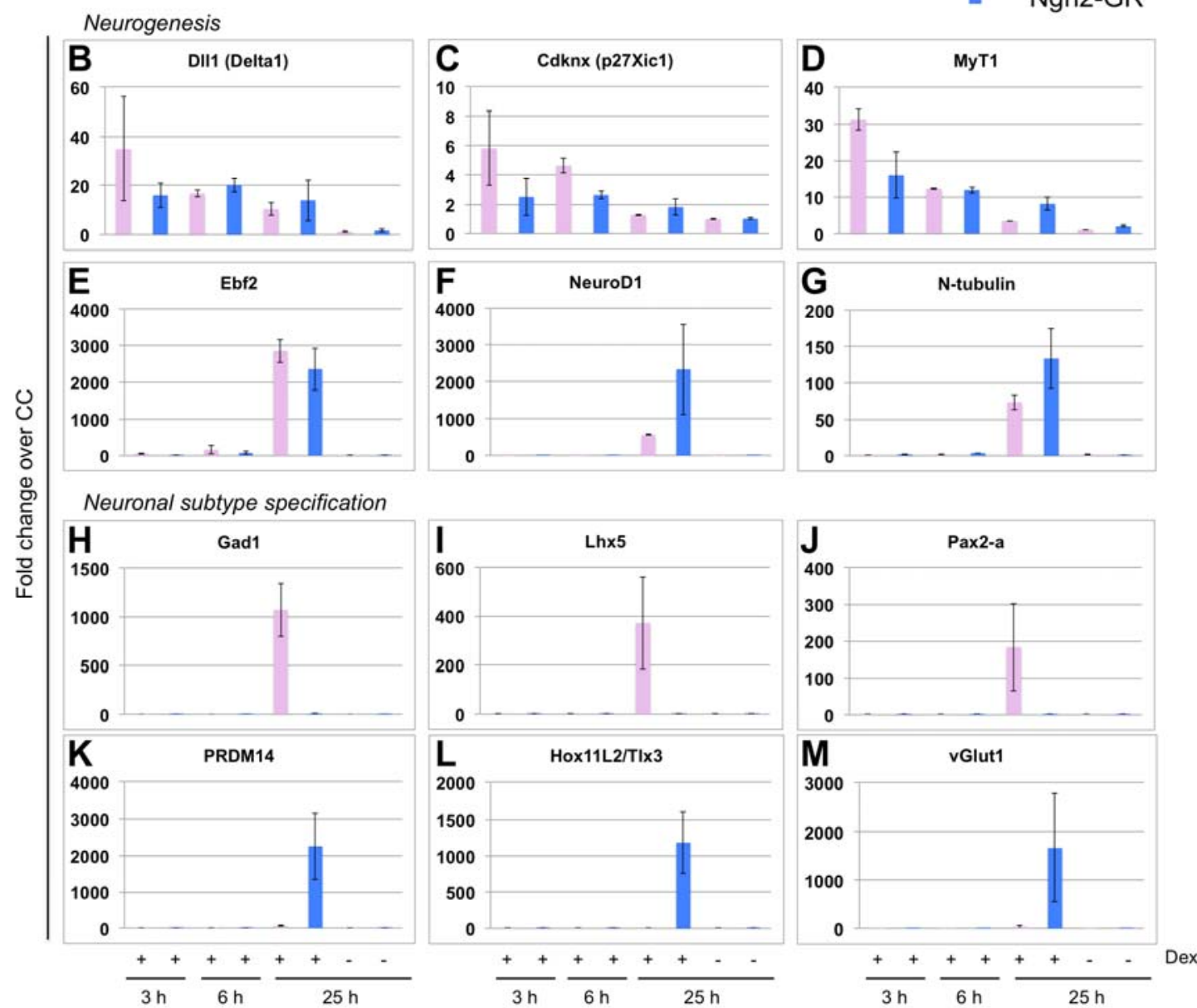

Figure 3.2 Ptf1a and Ngn2 promote general neurogenesis via activation of the same gene network.

Comparative temporal analysis of genes induced by Ptf1a-GR and Ngn2-GR mRNA in animal cap assays. (A) The indicated mRNAs (20 pg each) were injected into both blastomeres of two-cell stage embryos, animal caps excised at the blastula stage and treated with dexamethasone (Dex). Animal caps were cultured for 3,6 or $25 \mathrm{~h}$, which corresponds to stage 9,10 and 23 of sibling embryos. Total RNA was isolated and marker gene expression analysed by the Nanostring nCounter system. (B-M) Shown is the averaged fold change over uninjected control caps (CC) of two independent experiments. Error bars represent the standard error of the mean (+/-SEM). Note the different scales in each diagram. Shown are graphs of selected genes, for a full list see Appendix 6.1.1. 


\subsection{Ptf1a is able to drive both general neurogenesis and neuronal subtype specification when induced after neural tube closure}

Multiple proneural bHLH genes have been identified in vertebrates, including $X$. laevis (Lee, 1997). Analysis of the expression pattern of the $X$. laevis proneural genes reveals expression in overlapping and distinct domains in the CNS (Fig. 1.3) (Nieber et al., 2009; Kim et al., 1997; Kanekar et al., 1997; Ferreiro et al., 1993; Turner and Weintraub, 1994), suggesting that the proneural genes may have discrete activities in generating specific neuronal identities. By whole mount in situ hybridization, the earliest specific expression of Ptf1a is detected at stage 19 in a single longitudinal domain within the neural folds (Afelik et al., 2006). At late tadpole stages, Ptf1a is specifically expressed in the hindbrain, the spinal cord and the retina as well as in the ventral and dorsal pancreatic bud (Afelik et al., 2006). Previous experiments had shown that Ptf1a-GR, when activated at gastrula stage, is able to drive general neurogenesis (Dullin et al., 2007; Hedderich, 2008). To analyse, if the expression of Ptf1a at stage 19 may indicate an early physiological function in inducing general neurogenesis, Ptf1a-GR mRNA was injected into one blastomere of two-cell stage embryos and protein activity induced at stage 19 (Fig. 3.3 A).

Gene expression analysis by in situ hybridization showed that in contrast to induction of Ptf1a-GR activity during early gastrula stages, induction at stage 19, when the ectoderm has obtained an epidermal fate, did not lead to ectopic N-tubulin or Gad1 expression (Fig. 3.3 B and C). However, the corresponding sections through the hindbrain revealed increased expression of both marker genes on the injected side (Fig. $3.3 \mathrm{~B}^{\prime}$ and $\mathrm{C}^{\prime}$ ). This result demonstrates that Ptf1a-GR, when induced at a time when endogenous Ptf1a is present, is still able to promote general neurogenesis and a GABAergic interneuronal identity within the neural tube. Moreover, Ptf1a-GR activated at stage 19 was still able to inhibit Hox11L2/TIX3 expression on the injected side of the embryo (Fig. 3.3 $\left.D, D^{\prime}\right)$. 


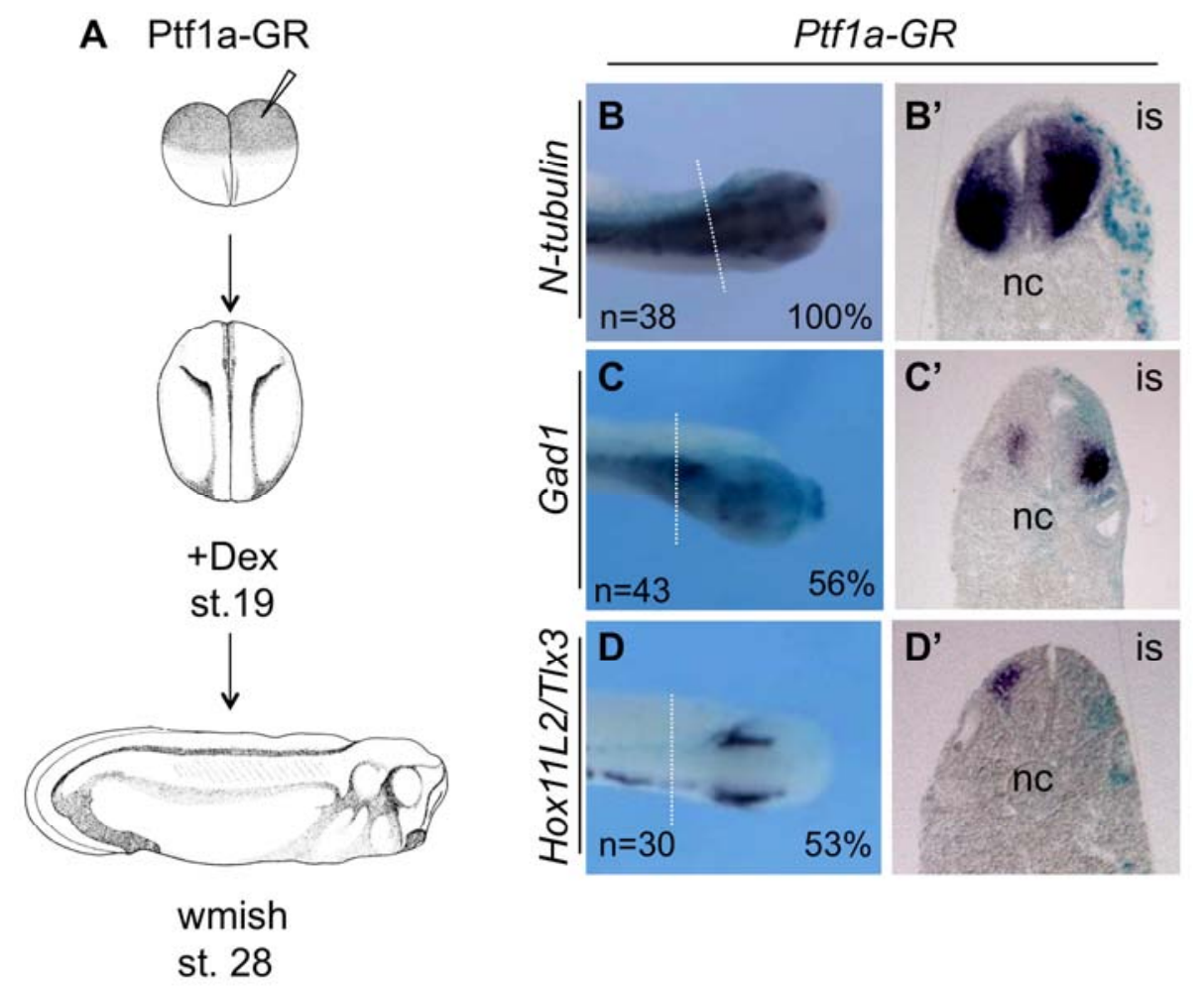

Figure 3.3 Ptf1a is able to drive general neurogenesis and neuronal subtype specification when induced after neural tube closure. (A) Ptf1a-GR mRNA (20 pg) was injected into one blastomere of two-cell stage embryos and induced at stage 19 with dexamethasone (Dex). (B-D) Gene expression was analysed by whole mount in situ hybridization (wmish) of stage $28 X$. laevis embryos using markers indicated on the left side. (B-D) Dorsal view of the head region with the injected side at the top and (B'$\left.D^{\prime}\right)$ the corresponding transversal sections through the hindbrain at the level of the otic vesicle are shown with the injected side to the right. nc, notochord; is, injected side

\subsection{Ptf1a is maternally expressed in X. laevis}

The ability of Ptf1a to induce general neurogenesis before neural tube closure might reflect an early physiological activity. To evaluate if Ptf1a might be expressed earlier than detected by in situ hybridization, a semi-quantitative RTPCR of different developmentally staged $X$. laevis embryos analysed for Ptf1a transcripts was performed, surprisingly revealing maternal expression of Ptf1a (Fig. 3.4 A). Ptf1a transcripts were maintained at constant levels until late gastrula stage (stage 11), demonstrating that Ptf1a is actually expressed when the first neurons are being born. From early neurula stages (stage 13) onwards, the level of Ptf1a expression decreased and increased again after neural tube closure (stage 20). Gene expression analysis by in situ hybridization revealed that the maternal Ptf1a transcripts are restricted to the animal half of the embryo at early cleavage stages (stage 2 and stage 6) (Fig. 3.4 B and C). At early 
gastrula stage (stage 10.5), Ptf1a transcripts were excluded from the blastoporus (Fig. 3.4 D). Consistent with previous reports (Afelik et al., 2006), at later neurula stages, weak and diffuse Ptf1a expression was detectable in a single longitudinal domain within the neural folds, which became stronger after neural tube closure (Fig. $3.4 \mathrm{E}$ and F). To exclude unspecific background staining, particularly at the early stages, in situ hybridization with a Ptf1a sense RNA probe was performed (Fig. 3.4 G - K). In all stages, no specific signal was detected, demonstrating that staining with the Ptf1a antisense RNA probe was specific.

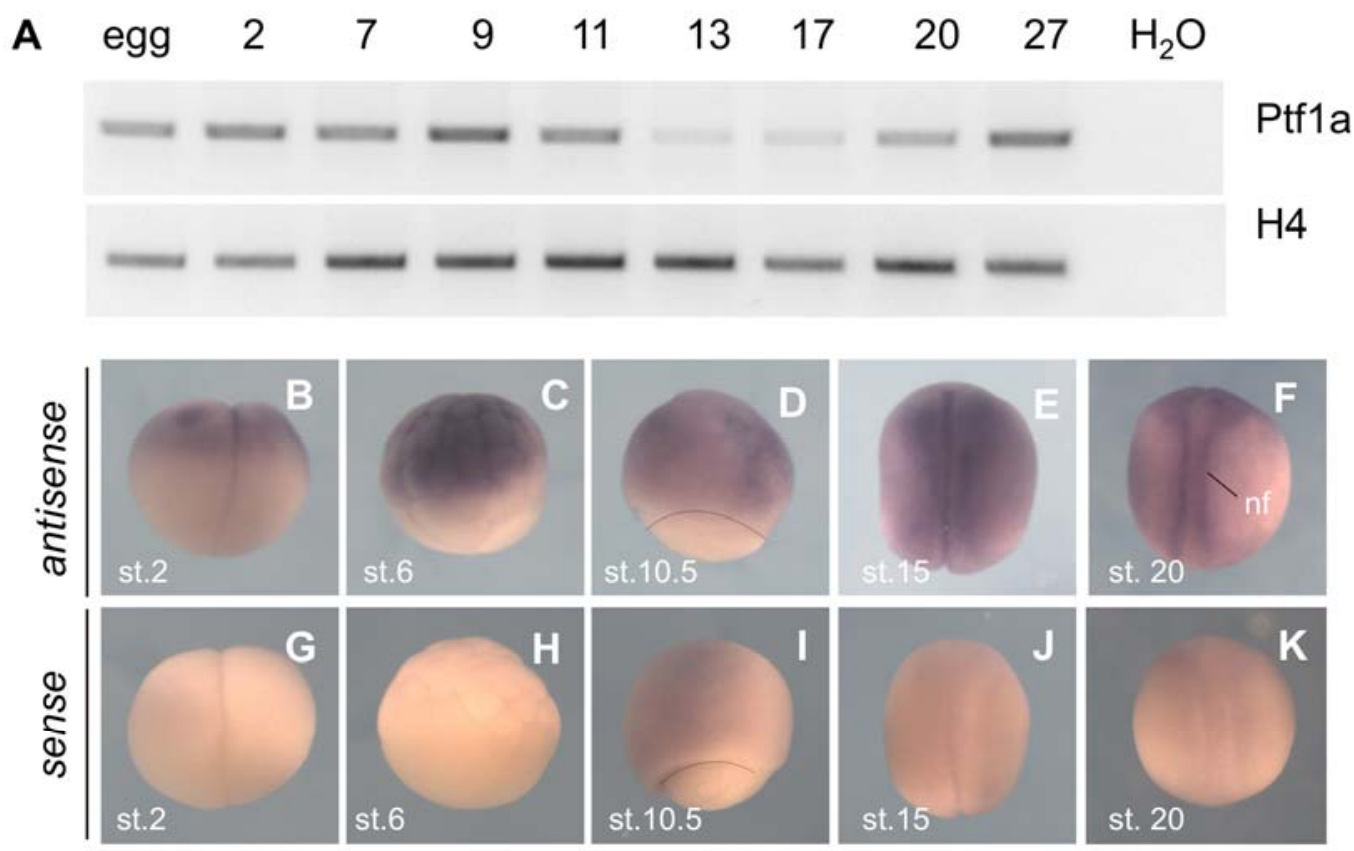

Figure 3.4 Spatial and temporal Ptf1a expression in $X$. laevis.

(A) Temporal Ptf1a expression. Ptf1a expression was analysed by semi-quantitative RT-PCR. Expression levels were shown by histone 4 (H4) expression. (B-M) Spatial Ptf1a expression. Ptf1a expression pattern was analysed by whole mount in situ hybridization using (B-F) antisense RNA or (G-K) sense RNA. Shown is the lateral view of the embryo in $B, C, D, G, H, I$ and the dorsal view in $E, F, J$ and $K$. Dotted line in $D$ and I marks the blastoporus. nf, neural folds

\subsection{Ptf1a is expressed in the inner ventricular zone of the hindbrain and neural tube}

To further determine if Ptf1a endogenously might be involved in general neurogenesis in the developing embryo, it was evaluated, if Ptf1a is expressed in neural progenitor cells that do not express members of the well-characterised neuronal determination factor family Neurogenin (Fig. 3.5 A-C). Therefore, a 
double in situ hybridization of stage 29 X. laevis embryos for Ptf1a and the three Neurogenins (Ngn1, Ngn2 and Ngn3) (Ma et al., 1996; Nieber et al., 2009) was performed. The gene expression analysis revealed that Ptf1a and Ngn1 (Fig. $3.5 \mathrm{~B}, \mathrm{~B}^{\prime}$ ) and $\mathrm{Ngn} 3$ (Fig. $3.5 \mathrm{C}, \mathrm{C}^{\prime}$ ) were expressed in non-overlapping territories of the hindbrain. In contrast, Ptf1a and Ngn2 (Fig. 3.5 A, A') were coexpressed in a restricted domain of the hindbrain that will give rise to GABAergic interneurons, which is consistent with findings in the mouse (Henke et al., 2009b). However, only Ptf1a transcripts were detected in the inner ventricular zone where the proliferating precursor cells are located, whereas the expression domain of $\mathrm{Ngn} 2$ is found more lateral in the intermediate zone (Fig. 3.5 A, $\mathrm{A}^{\prime}$ ), being compatible with the idea, that Ptf1a functions as a proneural factor activating general neurogenesis in the inner ventricular zone of the hindbrain.
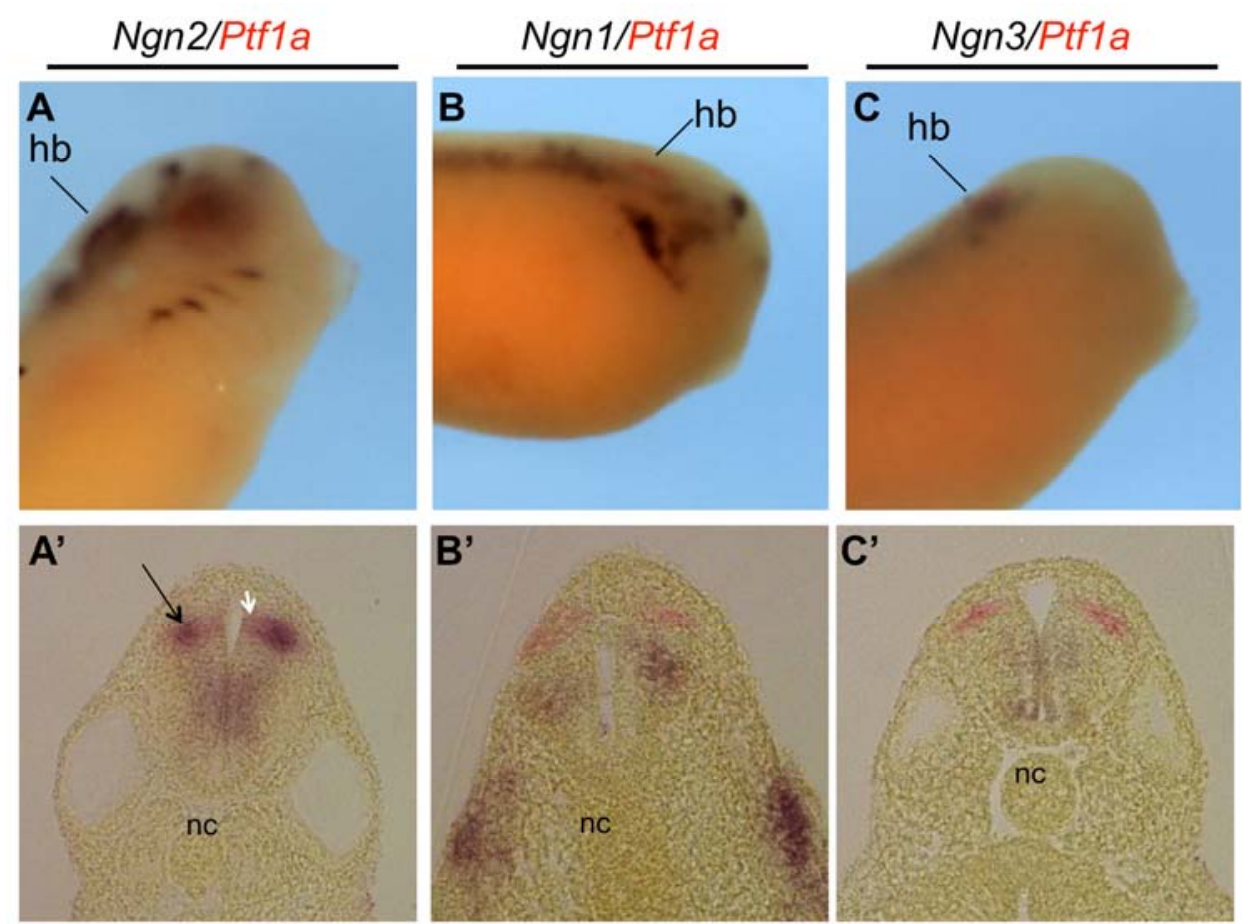

Figure 3.5 Ptf1a is expressed in the inner ventricular zone of the hindbrain.

(A-C) Double whole mount in situ hybridization of stage 29 X. laevis embryos for Ptf1a (red) and Ngn1, Ngn2 and Ngn3 (purple). (A-C) Lateral view of the head region and $\left(A^{\prime}-C^{\prime}\right)$ the corresponding transversal section through the hindbrain at the level of the otic vesicle. Black arrow indicates the region, where Ptf1a and Ngn2 are co-expressed. The ventricular zone of the hindbrain expressing Ptf1a is indicated by the white arrow head. nc, notochord, hb, hindbrain 


\subsection{The neuronal subtype-inducing activity of Ptf1a is dominant over Ngn2}

The previous result (Fig. 3.5) shows that Ptf1a and Ngn2 are co-expressed in the region of the hindbrain that gives rise to GABAergic inhibitory neurons. However, Ngn2 and Ptf1a promote distinct neuronal subtype identities in postmitotic neurons (Fig. 3.1) (Dullin et al., 2007; Hedderich, 2008). To analyse the neuronal subtype inducing-activity of Ptf1a and Ngn2 when co-expressed, Ptf1a-GR and Ngn2-GR mRNA were injected alone or together into both blastomeres of two-cell stage embryos. Animal caps were excised at blastula stage and the GR fusion proteins induced by dexamethasone (Fig. 3.6 A). When the control embryos reached stage 23, total RNA was isolated and marker gene expression analysed by quantitative real-time RT-PCR (Fig. 3.6 A).

Overexpression of Ptf1a-GR and Ngn2-GR mRNA alone or in combination induced $N$-tubulin expression compared to uninjected control caps (Fig. 3.6 B). Consistent with previous results, Ptf1a-GR but not Ngn2-GR, strongly induced the expression of Gad1 (Fig. $3.6 \mathrm{C}$ ), whereas Ngn2-GR activated the marker expression of Hox11L2/T/x3 (Fig. 3.6 D). Upon coexpression of both bHLH transcription factors, Ptf1a-GR mRNA was still sufficient to activate Gad1 expression (Fig. 3.6 C). In fact, the induction of Gad1 by Ptf1a-GR in combination with Ngn2-GR mRNA was even stronger than compared to Ptf1a-GR alone (Fig. $3.6 \mathrm{C}$ ). In contrast, the induction of Hox11L2/TIx3 by Ngn2-GR upon co-injection of Ptf1a-GR mRNA was abolished (Fig. 3.6 D). These results suggest that the GABAergic neuronal subtypeinducing activity of Ptf1a is dominant over that from Ngn2. 


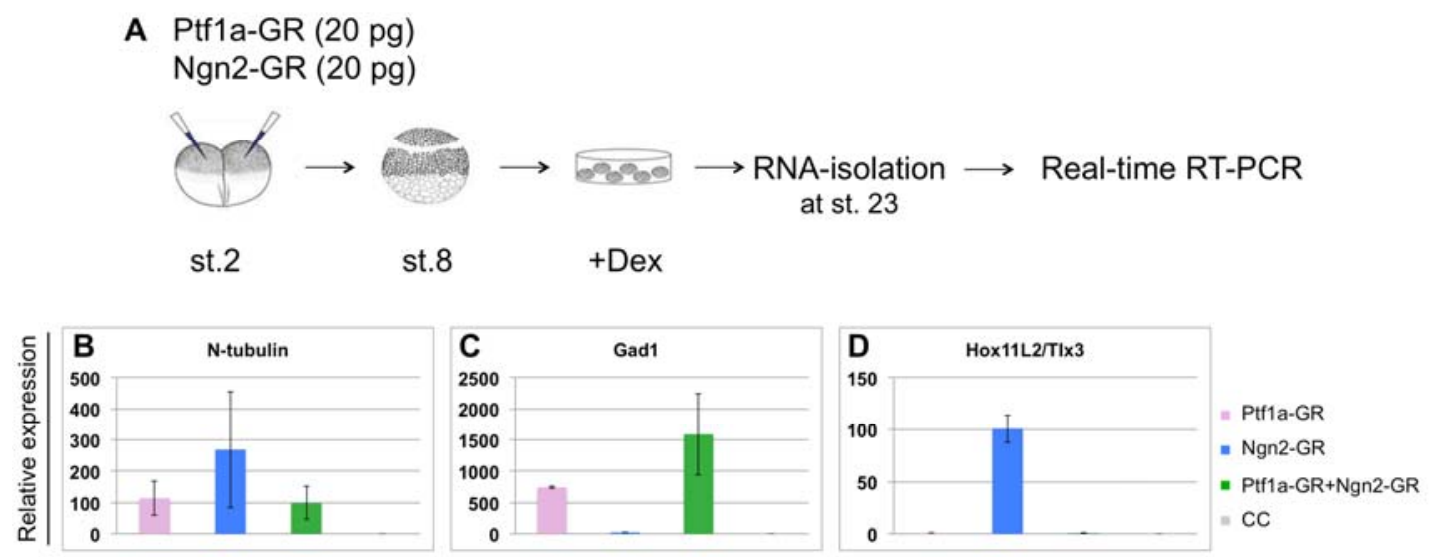

Figure 3.6 The neuronal subtype-inducing activity of Ptf1a is dominant over Ngn2.

The ability of Ngn2 together with Ptf1a to induce general neurogenesis and neuronal subtype identity was analysed in animal cap assays. (A) The indicated mRNAs (20 pg each) were injected into both blastomeres of two-cell stage embryos, animal caps excised at the blastula stage and treated with dexamethasone (Dex) until sibling embryos reached stage 23. Total RNA was isolated and marker gene expression analysed by quantitative real-time RT-PCR. (B-D) Shown is the average of two independent experiments. Error bars represent the standard error of the mean $(+/-$ SEM).

\subsection{The bHLH domain of Ptf1a is not essential for GABAergic interneuronal subtype specification}

As shown above, Ngn2 and Ptf1a drive general neurogenesis of neural progenitors through the same cascade of target genes, however, they confer distinct neuronal subtype identities in the post-mitotic neurons. In order to analyse if the shared and unique activities of Ptf1a and Ngn2 are regulated by sequences within their bHLH domains, a chimeric construct was created by swapping the Ptf1a bHLH domain for that of Ngn2 (chimeric Ptf1a-GR, Fig. 3.7 B) (Hedderich, 2008). Previous analysis of a restricted set of marker genes implicated that the bHLH domain of Ptf1a might not be sufficient to impart specificity during the induction of GABAergic interneurons (Hedderich, 2008). To analyse the ability of the chimeric Ptf1a-GR to drive general neurogenesis and to specify a GABAergic interneuronal subtype in detail, quantitative digital multiplexed gene expression analysis using the NanoString nCounter system was performed (Fig. 3.7 and Appendix 6.1.2). Ptf1a-GR, Ngn2-GR or chimeric Ptf1a-GR mRNA were injected into both blastomeres of two-cell stage embryos. Animal caps were excised at blastula stage, proteins induced and cultivated until sibling embryos reached stage 23 . Total RNA was isolated and quantitative Nanostring analysis performed (Fig. 3.7 and Appendix 6.1.2). 
To first evaluate the role of the bHLH domain in promoting general neurogenesis, genes involved in general neuronal differentiation were analysed. Similar to Ptf1a-GR and Ngn2-GR, overexpression of the chimeric Ptf1a-GR mRNA was sufficient to induce neuronal differentiation factors such as Ebf2 (Fig. 3.7 C), NeuroD1 (Fig. 3.7 D), Ebf3 (Appendix 6.1.2) and NeuroD4 (Appendix 6.1.2) as well as $N$-tubulin expression (Fig. 3.7 E). Furthermore, the chimeric Ptf1a-GR was also able to induce the expression of the cell-cycle inhibitor Pak3 (Appendix 6.1.2). In respect to neuronal subtype specificity, the chimeric Ptf1a-GR induced a robust expression of Gad1 (Fig. 3.7 F), a marker indicative of GABAergic neurons. However, the induction compared to wild-type Ptf1a-GR was reduced about 2.5-fold. Overexpression of the chimeric Ptf1a-GR mRNA also activated the expression of transcription factors involved in GABAergic interneuronal subtype specification such as Pax2 (Fig. $3.7 \mathrm{H}$ ), Lhx5 (Fig. 3.7 G), $L h x 1$ (Appendix 6.1.2) and Lbx1 (Appendix 6.1.2), but compared to wild-type Ptf1a-GR the induction was strongly reduced, in particular for Pax2 and Lbx1. However, chimeric Ptf1a-GR-mediated induction of gene expression was considerably stronger than background expression levels observed upon Ngn2-GR mRNA overexpression. Interestingly like Ngn2-GR, microinjection of the chimeric Ptf1a-GR mRNA was sufficient to activate the expression of Prdm14 (Fig. 3.7 I), Hox11L2/TIx3 (Fig. $3.7 \mathrm{~J}$ ) and Vglut1 (Fig. $3.7 \mathrm{~K}$ ), albeit compared to Ngn2-GR the induction was also greatly reduced.

Taken together, the quantitative Nanostring analysis demonstrated that the specificity of Ptf1a to drive general neurogenesis is not based on the function of its individual bHLH domain. Furthermore, the bHLH domain of Ptf1a is not essential for the determination of a GABAergic interneuronal subtype as the chimeric Ptf1a was still sufficient to activate marker gene expression indicative of a GABAergic interneuronal identity, even though the induction was greatly reduced. This suggests that the specification of GABAergic interneurons by Ptf1a is not only conferred through the DNA binding domain via the bHLH domain but likely also due to flanking sequences, which may impart specificity through interaction with other proteins. 
A Ptf1a-GR $(20 \mathrm{pg})$

Ngn2-GR $(20 \mathrm{pg})$

chimeric Ptf1a-GR (20 pg)

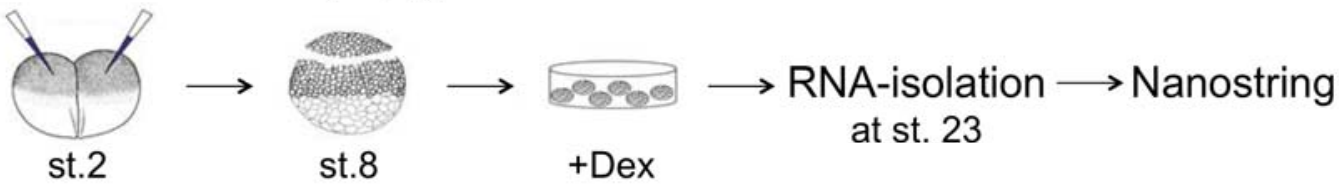

B Ptf1a wt (270 aa)
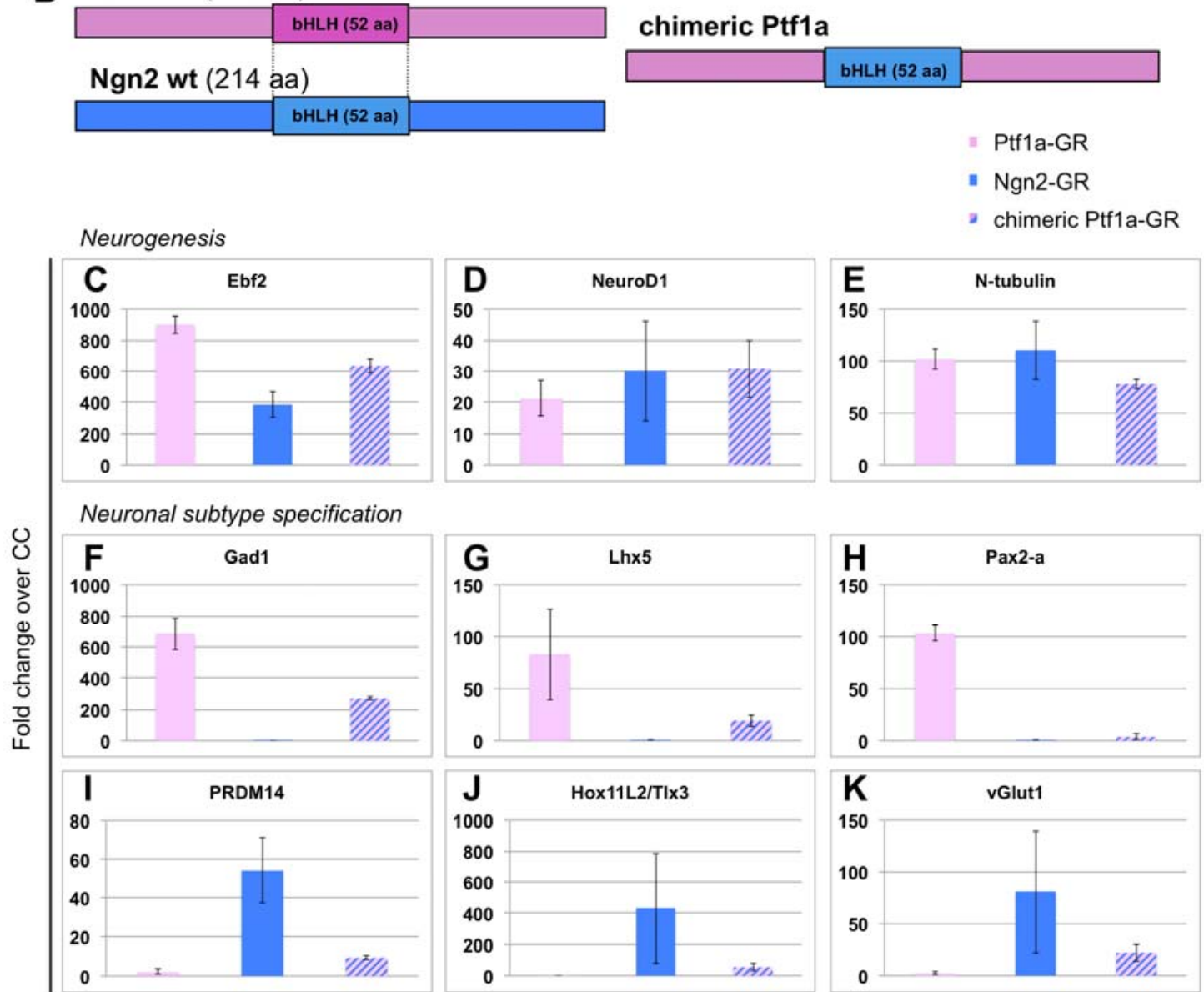

Figure 3.7 The bHLH domain of Ptf1a is not essential for GABAergic interneuron subtype specification.

The ability of Ngn2, Ptf1a or a chimeric construct of Ptf1a (chimeric Ptf1a) to induce general neurogenesis and neuronal subtype identity was analysed in animal cap assays. (A) The indicated mRNAs (20 pg each) were injected into both blastomeres of two-cell stage embryos, animal caps excised at the blastula stage and treated with dexamethasone (Dex) until sibling embryos reached stage 23. Total RNA was isolated and marker gene expression analysed by Nanostring. (B) Scheme of the chimeric Ptf1a construct, in which the bHLH of Ptf1a was swapped with that of Ngn2. (C-K) Shown is the averaged fold change over uninjected control caps (CC) of two independent experiments. Error bars represent the standard error of the mean (+/SEM). Note the different scales in each diagram. Shown are graphs of selected genes, for a full list see Appendix 6.1.2. 


\subsection{Interaction of Ptf1a with $\mathrm{Su}(\mathrm{H})$ is not required for the general neurogenesis-inducing activity of Ptf1a}

The previous result suggested that also sequences outside the bHLH domain of Ptf1a are responsible for its specificity in neuronal subtype specification. Studies in mouse and chick demonstrated that the C-terminus of Ptf1a is essential for Ptf1a to promote GABAergic interneuronal subtype identity (Hori et al., 2008). The Ptf1a C-terminus contains a C1- and C2-domain, which were shown to be required for the interaction with $\mathrm{Rbp}-\mathrm{j}(\mathrm{Su}(\mathrm{H})$ in $X$. laevis) or its paralogue Rbp-I (Beres et al., 2006). The interaction with Rbp-j can be abolished through a point mutation in the C2-domain. In the mouse and chick neural tube, this mutant Ptf1a lost the ability to promote GABAergic inhibitory interneurons (Hori et al., 2008). Previous studies in $X$. laevis investigating the effect of the interaction between Ptf1a and $\mathrm{Su}(\mathrm{H})$ on Ptf1a function, revealed that both $\mathrm{C}$-domains must be mutated by an exchange of one amino acid (Ptf1a ${ }^{\text {W224A/W242A }}$, Hedderich, 2008) (Fig. 3.8 B) to abolish the ability of Ptf1a to induce a GABAergic interneuronal identity (Hedderich, 2008). To further analyse the importance of these mutations on Ptf1a activity, the animal cap assay coupled with quantitative Nanostring analysis was utilized. Ptf1a-GR or Ptf1a ${ }^{W 224 A}$ W242A $-G R$ mRNA were injected into both blastomeres of two-cell stage embryos, animal caps excised and protein activity induced at blastula stage (Fig. $3.8 \mathrm{~A}$ ). The explants were collected $6 \mathrm{~h}$ and $32 \mathrm{~h}$ after dexamethasone induction, total mRNA was isolated and analysed by Nanostring (Fig. 3.8 and Appendix 6.1.3).

Similar to Ptf1a-GR wild-type, microinjection of Ptf1a ${ }^{W 224 A W 242 A}-G R$ mRNA activated genes involved in general neurogenesis. The genes DIl1 (Fig. 3.8 C), Cdknx (Fig. 3.8 D) and Myt1 (Fig. 3.8 E) were already induced after $6 \mathrm{~h}$, while the other neuronal differentiation factors such as NeuroD1 (Fig. 3.8 F), Ebf2 (Appendix 6.1.3), Ebf3 (Appendix 6.1.3), NeuroD4 (Appendix 6.1.3) as well as $N$-tubulin (Fig. $3.8 \mathrm{G}$ ) were activated after $32 \mathrm{~h}$ of protein activity initiation. However, in regard to neuronal subtype specificity, only the wild-type Ptf1a-GR but not the Ptf1a ${ }^{W 224 A / W 242 A}-G R$ mRNA induced a robust activation of the GABAergic interneuronal marker genes Gad1 (Fig. 3.8 I), Pax2 (Fig. $3.8 \mathrm{~J})$, Lhx1 (Appendix 6.1.3), Lhx5 (Fig. 3.8 K) and $L b x 1$ (Appendix 6.1.3) after $32 \mathrm{~h}$ of protein induction. In contrast, Ptf1a ${ }^{W 224 A W 242 A}-G R$ but not Ptf1a-GR wild-type 
activated marker genes indicative of a glutamatergic excitatory phenotype such as Hox11L2/TIx3 (Fig. 3.8 M) and Vglut1 (Fig. 3.8 N) as well as Prdm14 (Fig. 3.8 L). As Ngn2, but not Ptf1a wild-type was shown in similar assays to promote such marker gene expression (Fig. 3.2), the expression of Ngn2 was evaluated.

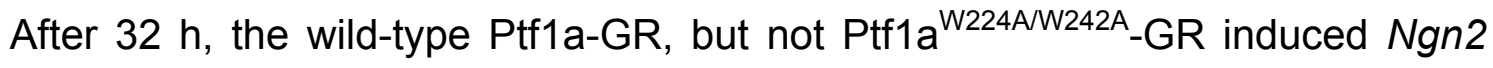
(Fig. $3.8 \mathrm{H}$ ) expression, which is consistent with the finding that Ngn2 is a direct target of the heterotrimeric Ptf1a complex in mouse (Henke et al., 2009b). In contrast, the other members of the Neurogenin family, Ngn1 and Ngn3, were differentially regulated. Both, Ptf1a-GR as well as Ptf1a ${ }^{W 224 A / W 242 A}-G R$ mRNA was able to induce Ngn1 (Appendix 6.1.3) but not Ngn3 (Appendix 6.1.3) expression.

In summary, the experiment supports the hypothesis that Ptf1a forms two distinct transcription complexes during $X$. laevis neurogenesis: a $\mathrm{Su}(\mathrm{H})$ independent one to drive general neurogenesis and to specify glutamatergic excitatory neurons and a $\mathrm{Su}(\mathrm{H})$-dependent one to specify GABAergic inhibitory interneurons. 
A Ptf1a-GR (20 pg)

Ptf1aW224AW242A-GR (20 pg)

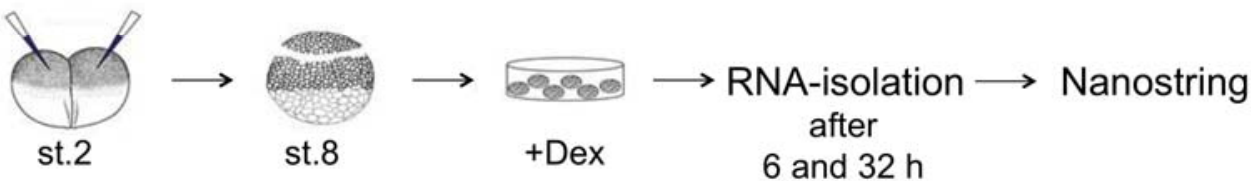

B Ptf1a wt (270 aa)

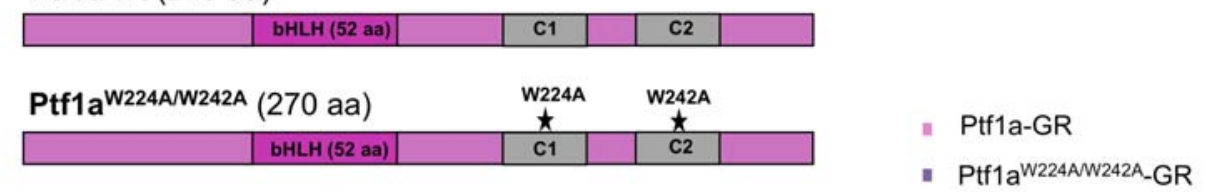

Neurogenesis
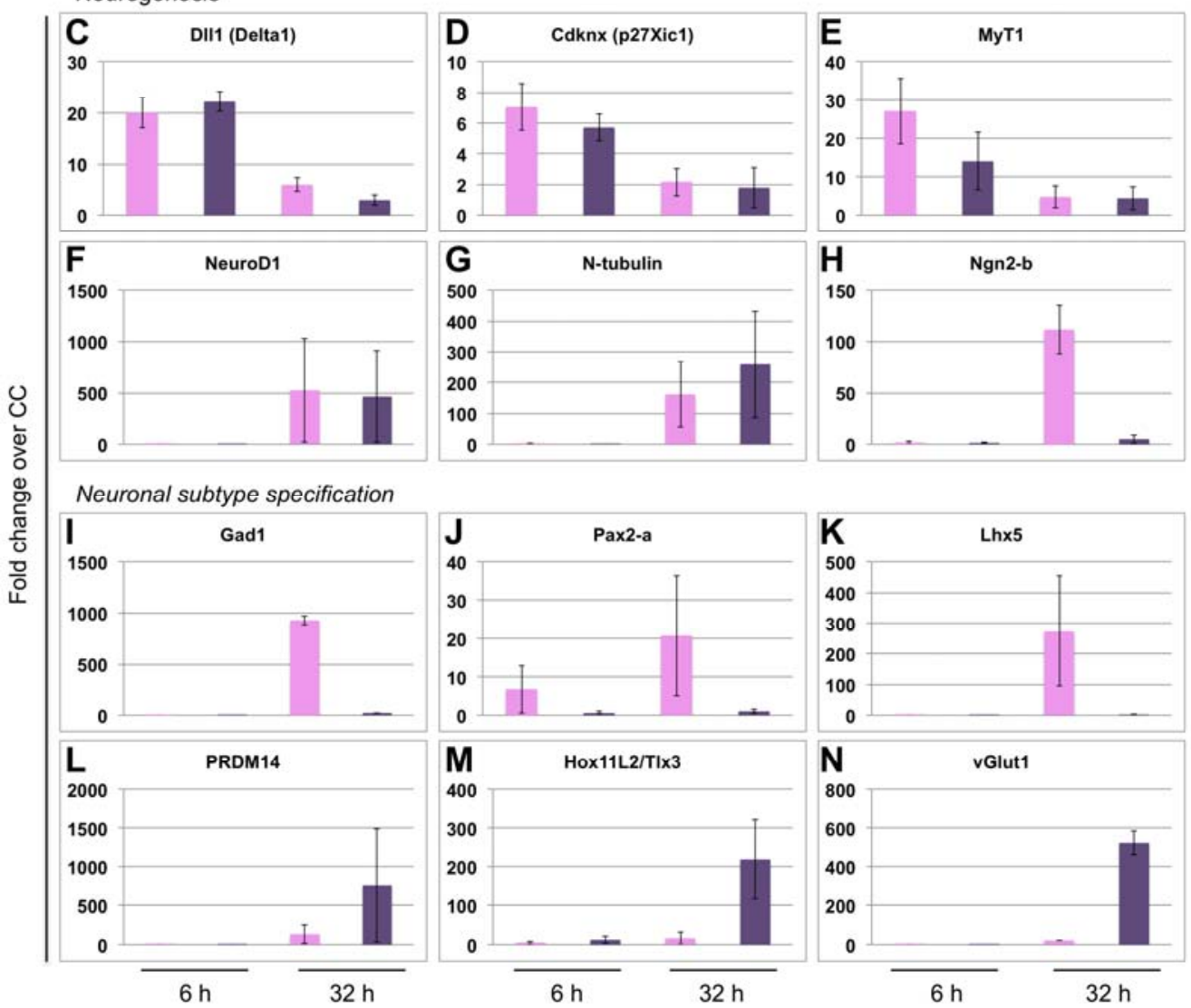

Figure 3.8 Interaction of Ptf1a with $\mathrm{Su}(\mathrm{H})$ is not required for the general neurogenesis-inducing activity of Ptf1a.

Comparative temporal analysis of wild-type Ptf1a and a mutant Ptf1a (Ptf1a ${ }^{\text {W224A/W242A }}$, containing two point mutations that disrupt the association with $\mathrm{Su}(\mathrm{H})$, to induce markers of general neurogenesis and neuronal subtype identity was analysed in animal cap assays. (A) The indicated mRNAs (20 pg each) were injected into both blastomeres of two-cell stage embryos, animal caps excised at the blastula stage and treated with dexamethasone (Dex) for 6 and $32 \mathrm{~h}$. Total RNA was isolated and marker gene expression analysed by Nanostring. (B) Scheme of the Ptf1a mutant $\left(\mathrm{Ptf} 1 \mathrm{a}^{\mathrm{W} 224 \mathrm{~A} / \mathrm{W} 242 \mathrm{~A}}\right)$ construct. At position 224 in the $\mathrm{C} 1$ domain and at position 242 in the C2 domain a tryptophan residue was exchanged for an alanine, which disrupts the interaction with $\mathrm{Su}(\mathrm{H})$. (C-N) Marker gene expression analysed by Nanostring. Shown is the averaged fold change over uninjected control caps (CC) of two independent experiments. Error bars represent the standard error of the mean (+/-SEM). Note the different scales in each diagram. Shown are graphs of selected genes, for a full list see Appendix 6.1.3. 


\subsection{Ptf1a is capable of interacting with $\mathrm{Su}(\mathrm{H})$ at early embryogenesis}

In mouse, the proneural gene Ngn2 was identified as a direct target gene that requires the heterotrimeric complex of Ptf1a (Henke et al., 2009b). It is likely that also in X. laevis, Ngn2 is a direct downstream target of Ptf1a. This is further supported by the co-expression of Ngn2 with Ptf1a in X. laevis in the intermediate layer of the hindbrain progenitor domain (Fig. 3.5) that gives rise to GABAergic interneurons. Interestingly, in the quantitative temporal expression analysis shown in Figure 3.8 (and Appendix 6.1.3), Ngn2 and other Ptf1a $\mathrm{Su}(\mathrm{H})$-dependent genes were activated only after $\mathrm{Su}(\mathrm{H})$-independent genes.

This raises the question as to what prevents the activation of $\mathrm{Ngn} 2$ and other trimeric complex target genes from being activated in an immediate early fashion by the Ptf1a heterotrimeric complex that contains $\mathrm{Su}(\mathrm{H})$ ? One possibility could be that the heterotrimeric Ptf1a complex only forms when cells obtain their neuronal subtype identity, because $\mathrm{Su}(\mathrm{H})$ is not present or is at low levels during the phase of general neurogenesis. Therefore, the temporal expression of $S u(H)$ transcripts during $X$. laevis development was analysed using total RNA from staged embryos (Fig. 3.9 A). Semi-quantitative RT-PCR analysis revealed that $S u(H)$ is maternally expressed and that $S u(H)$ transcripts were present at equal levels throughout all development stages tested.

The ability of in vitro synthesized Ptf1a to bind Su(H) (Hedderich, 2008; Beres et al., 2006) suggests that the interaction is direct and not dependant on post-translational modification. However, post-translational modifications may prevent their association. Therefore, in vivo co-immunoprecipitation (Co-IP) experiments were done using lysates of embryos injected with Myc-tagged wildtype (MT-Ptf1a-GR) or mutated Ptf1a (MT-Ptf1a ${ }^{\text {W224AW242A }}-$ GR) mRNA together with HA-tagged Su(H) mRNA (Su(H)-HA) (Fig. $3.9 \mathrm{~B}$ and $\mathrm{C}$ ) to analyse if the heterotrimeric Ptf1a complex is capable of forming at early gastrula stages, when only $\mathrm{Su}(\mathrm{H})$-independent Ptf1a target genes are activated. MT-Ptf1a-GR, but not MT-Ptf1a $a^{\text {W224A/W242A }}-G R$, which served as a negative control, was coimmunoprecipitated with Su(H)-HA (Fig. 3.9 C). To confirm the result, the Co-IP was also performed in the reverse direction (Fig. 3.9 B), showing that $\mathrm{Su}(\mathrm{H})-\mathrm{HA}$ was co-immunoprecipitated with MT-Ptf1a-GR, but not with MT-Ptf1a ${ }^{\text {W224A/W242A }}$ GR. The Co-IP results demonstrated that MT-Ptf1a-GR, but not MT- 
Ptf1a ${ }^{\text {W224A/W242A }}-G R$ is capable of interacting already at gastrula stages with $\mathrm{Su}(\mathrm{H})-\mathrm{HA}$. Thus, at least when co-expressed, the heterotrimeric Ptf1a complex can form.
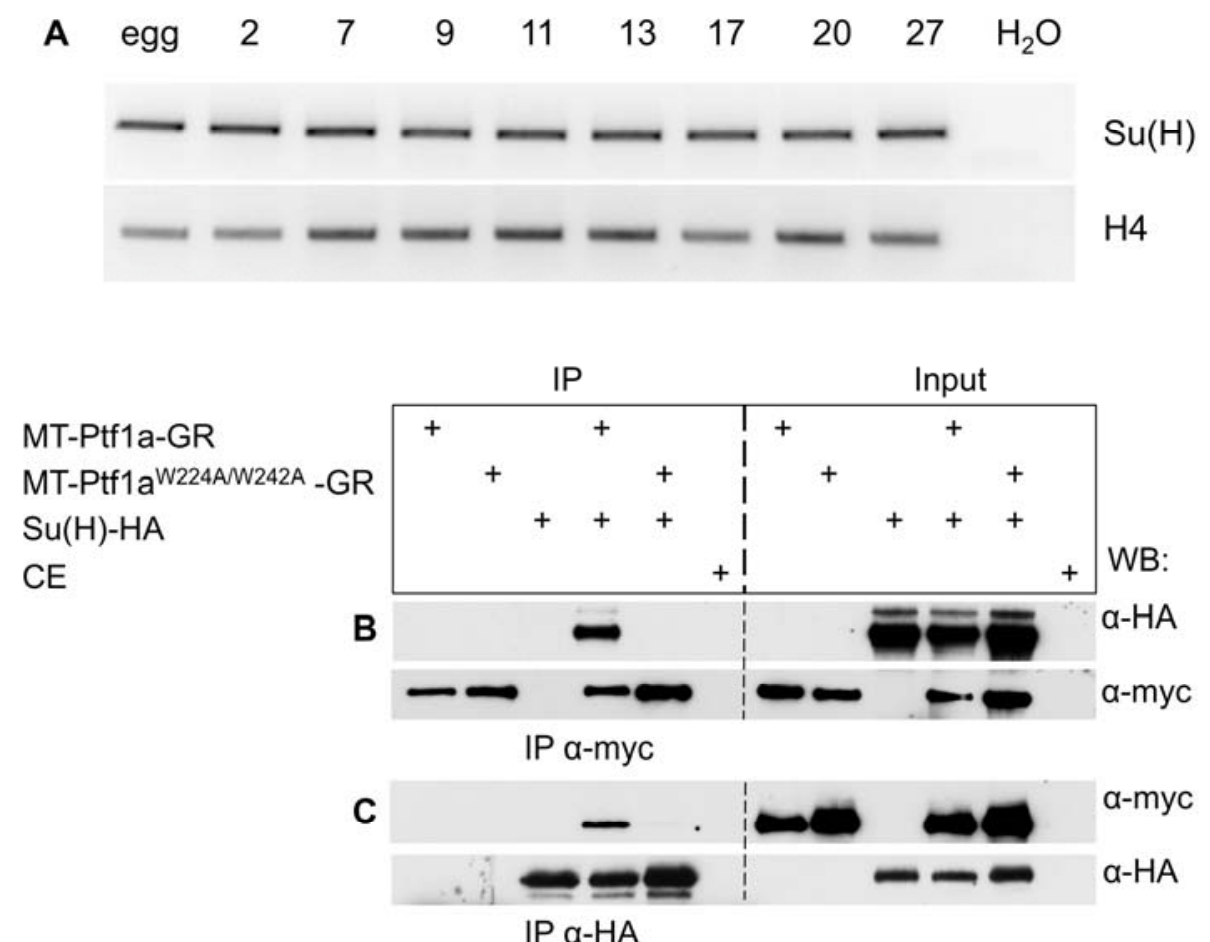

Figure 3.9 Ptf1a is capable of interacting with $\mathrm{Su}(\mathrm{H})$ at early embryogenesis.

(A) Temporal expression of $\mathrm{Su}(H)$. $\mathrm{Su}(H)$ expression levels were analysed by semiquantitative RT-PCR in whole $X$. laevis embryos. Expression levels were shown by histone H4. (B-C) Co-immunoprecipitation (Co-IP) of MT-Ptf1a-GR, MTPtf1a ${ }^{\text {W224A/W242A }}-\mathrm{GR}$ and $\mathrm{Su}(\mathrm{H})-\mathrm{HA}$ in $X$. laevis lysates. The mRNAs (250 pg each) were injected into both blastomeres of two-cell stage embryos. Co-IP was performed using embryo lysates from gastrula stage. (B-C) Co-IP experiments showing precipitation of $\mathrm{Su}(\mathrm{H})-\mathrm{HA}$ via MT-Ptf1a-GR (B) and precipitation of MT-Ptf1a-GR via Su(H)-HA (C).

\subsection{Co-expression of Ptf1a and $\mathrm{Su}(\mathrm{H})$ influences expression levels of Ptf1a Su(H)-dependent target genes}

Even though at early stages $\mathrm{Su}(H)$ transcripts are present and upon coinjection of Ptf1a and Su(H) mRNAs in X. laevis embryos, Ptf1a can bind $\mathrm{Su}(\mathrm{H})$, $\mathrm{Su}(\mathrm{H})$-dependent target gene activation is delayed following injection of Ptf1a$G R$ mRNA. One possibility could be that $\mathrm{Su}(\mathrm{H})$ protein availability might be limiting. This could occur for example through a competition between Notch-ICD and Ptf1a for $\mathrm{Su}(\mathrm{H})$-binding, which is mutually exclusive (Beres et al., 2006). The levels of $\mathrm{Su}(\mathrm{H})$ in the embryo were therefore increased by co-expression of $\mathrm{Su}(H)-G R$ mRNA together with Ptf1a-GR mRNA and the influence on marker 
gene expression determined and compared with injection of Ptf1a-GR mRNA alone. Su(H)-GR and Ptf1a-GR mRNA were injected alone or in combination into both blastomeres of two-cell stage embryos, animal caps excised at blastula stage and proteins activated for 3, 6, 9 and $25 \mathrm{~h}$. Total RNA was isolated and quantitative analysis performed with the Nanostring nCounter system (Fig. 3.10 A). Overexpression of $S u(H)-G R$ mRNA alone did not significantly increase the expression of any of the evaluated marker genes (Fig. 3.10 B-M). As shown previously, microinjection of Ptf1a-GR mRNA led to an early induction of the genes Myt1, DIl1 and Cdknx at $3 \mathrm{~h}$, which was not significantly altered by co-injection of $S u(H)$-GR mRNA (Fig. 3.10 B-D). Also similar to the previous analysis, the expression of the late neuronal differentiation markers such as NeuroD1 (Fig. 3.10 E), NeuroD4 (Appendix 6.1.4) and Ebf3 (Appendix 6.1.4) were strongly activated by Ptf1a-GR after $25 \mathrm{~h}$ of dexamethasone induction. However, co-injection of $S u(H)-G R$ mRNA led to a reduction in the expression of late neuronal differentiation markers, suggesting that neuronal differentiation is attenuated. This was further supported by the reduced expression of the post-mitotic marker $\mathrm{N}$-tubulin (Fig. 3.10 F) upon coinjection of $S u(H)-G R$ and Ptf1a-GR mRNA and an increase in the expression of Sox3 (Fig. $3.10 \mathrm{G}$ ), a marker for neural progenitor cells. Ptf1a-GR mRNA overexpression alone activated the genes Pax2, Lbx1, Lhx5, Lhx1 and Gad1 (Fig. $3.10 \mathrm{H}-\mathrm{K}$ and Appendix 6.1.4), which are indicative of a GABAergic interneurons in higher vertebrates (Helms and Johnson, 2003; Glasgow et al., 2005; Cheng et al., 2005), as well as Ngn2 expression (Fig. 3.10 L) after $25 \mathrm{~h}$ after dexamethasone induction. Co-injection of Su(H)-GR mRNA did not alter the onset of expression, however, the increase in $\mathrm{Su}(\mathrm{H})$ levels quantitatively influenced the induction of these genes. The induction of Gad1 (Fig. 3.10 H) and Pax2 (Fig. $3.10 \mathrm{I}$ ) was reduced upon co-injection of $S u(H)-G R$ mRNA, while the expression of $L h x 5$ (Fig. $3.10 \mathrm{~J}$ ) and $L m \times 1 b$ (Appendix 6.1.4) was unaffected. In contrast, co-expression of $S u(H)-G R$ mRNA led to a strong increase in expression of Lhx1 (Fig. 3.10 K), Ngn2 (Fig. 3.10 L) and Lbx1 (Appendix 6.1.4). Moreover, the expression of the pancreatic protein disulfide protein isomerase (Pdia2) was analysed (Fig. 3.10 M), which is a direct $\mathrm{Su}(\mathrm{H})$ dependent target gene of Ptf1a in the murine pancreas. Microinjection of Ptf1aGR mRNA alone was not sufficient to induce the expression of Pdia2, whereas 
together with $\mathrm{Su}(H)-G R$ mRNA Pdia2 expression was already activated (20fold) after $6 \mathrm{~h}$ of dexamethasone induction (Fig. 3.10), a time point at which Pdia2 normally is not present in the developing embryo. In summary, elevated $\mathrm{Su}(\mathrm{H})$ levels together with Ptf1a inhibited neuronal differentiation of progenitor cells, while the induction of $\mathrm{Su}(\mathrm{H})$-dependent target genes was either increased, decreased or not altered.

A Ptf1a-GR $(20 \mathrm{pg})$

$\mathrm{Su}(\mathrm{H})-\mathrm{GR}(40 \mathrm{pg})$

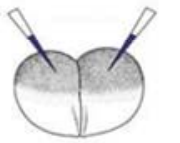

st.2

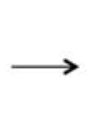

st.8

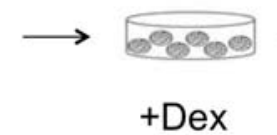

+ Dex $\longrightarrow$ RNA-isolation $\longrightarrow$ Nanostring after

$3,6,9,25 \mathrm{~h}$

Su(H)-independent
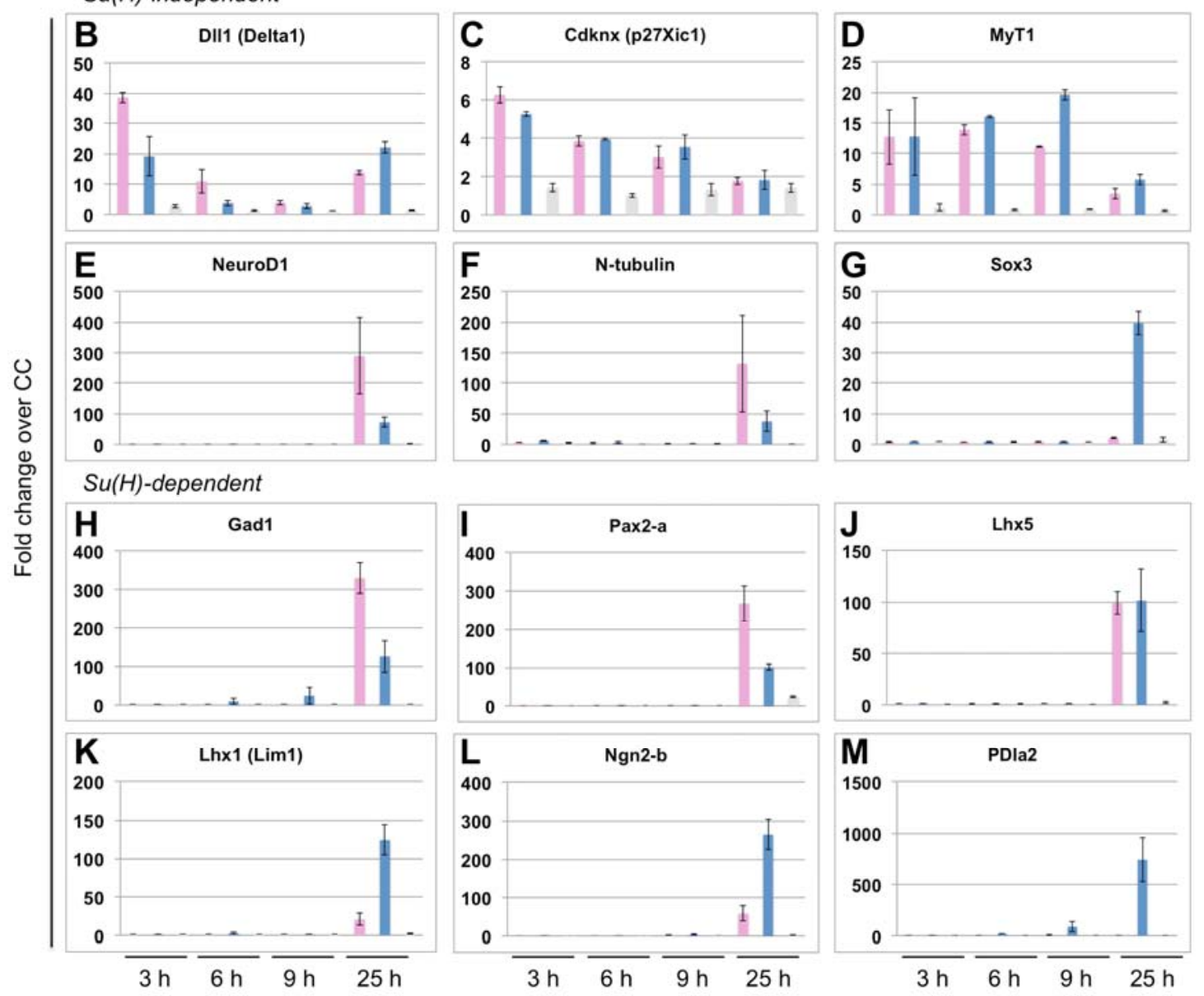

Figure 3.10 Co-expression of Ptf1a and $\mathrm{Su}(\mathrm{H})$ influences expression levels of Ptf1a Su(H)-dependent target genes.

Comparative analysis of the activity of Ptf1a-GR alone and together with $\mathrm{Su}(\mathrm{H})-\mathrm{GR}$. (A) Ptf1a-GR (20 pg) and Su(H)-GR (40 pg) mRNAs were injected into both blastomeres of two-cell stage embryos, animal caps excised at the blastula stage and treated with dexamethasone (Dex). Animal caps were cultured for $3,6,9$ or $25 \mathrm{~h}$, which 
corresponds to stage 9,10, 10.5 and 23 of sibling embryos. RNA was isolated and gene expression analysed by Nanostring. (B-M) Shown is the averaged fold change over uninjected control caps (CC) of two independent experiments. Error bars represent the standard error of the mean (+/-SEM). Note the different scales in each diagram. Shown are graphs of selected genes, for a full list see Appendix 6.1.4.

\subsection{Temporal expression analysis of genes induced by Ptf1a, Ptf1a ${ }^{\text {W224A/W242A }}$ and Ngn2 by RNA-sequencing}

To gain insight into the genetic network downstream of Ptf1a during general neurogenesis and neuronal subtype specification, a comparative temporal expression analysis of gene induction was performed in animal caps using whole transcriptome RNA-sequencing. To discriminate between $\mathrm{Su}(\mathrm{H})$ dependent and Su(H)-independent targets, both Ptf1a-GR and Ptf1a ${ }^{\text {W224A/W242A }}$ GR were used. Ngn2-GR was also included in this study to identify common proneural and unique neuronal subtype inducing activities.

For the whole transcriptome approach, Ptf1a-GR, Ptf1a ${ }^{W 224 A / W 242 A}-G R$ or Ngn2-GR mRNA were injected into both blastomeres of two-cell stage embryos, animal caps excised and treated with dexamethasone for 6 or $25 \mathrm{~h}$. After total RNA isolation, complementary DNA was prepared and paired-end deep sequencing was performed using an Illumina HiSeq 2000 sequencing system (more than 15 million reads per sample) to evaluate the transcriptome of the different explants (Fig. 3.12 A). To align the sequencing reads, the transcript reads were first mapped to the $X$. tropicalis $(X t)$ transcriptome as the genome is sequenced using the Bowtie2 program allowing three mismatches. Additionally, the transcript reads were also aligned to selected sequences of the $X$. laevis $(X I)$ unigene database, representing genes that were not found in the $X$. tropicalis transcriptome. In total, $74.7 \%$ of the sequencing-reads could be assigned using this approach (Fig. 3.11). 


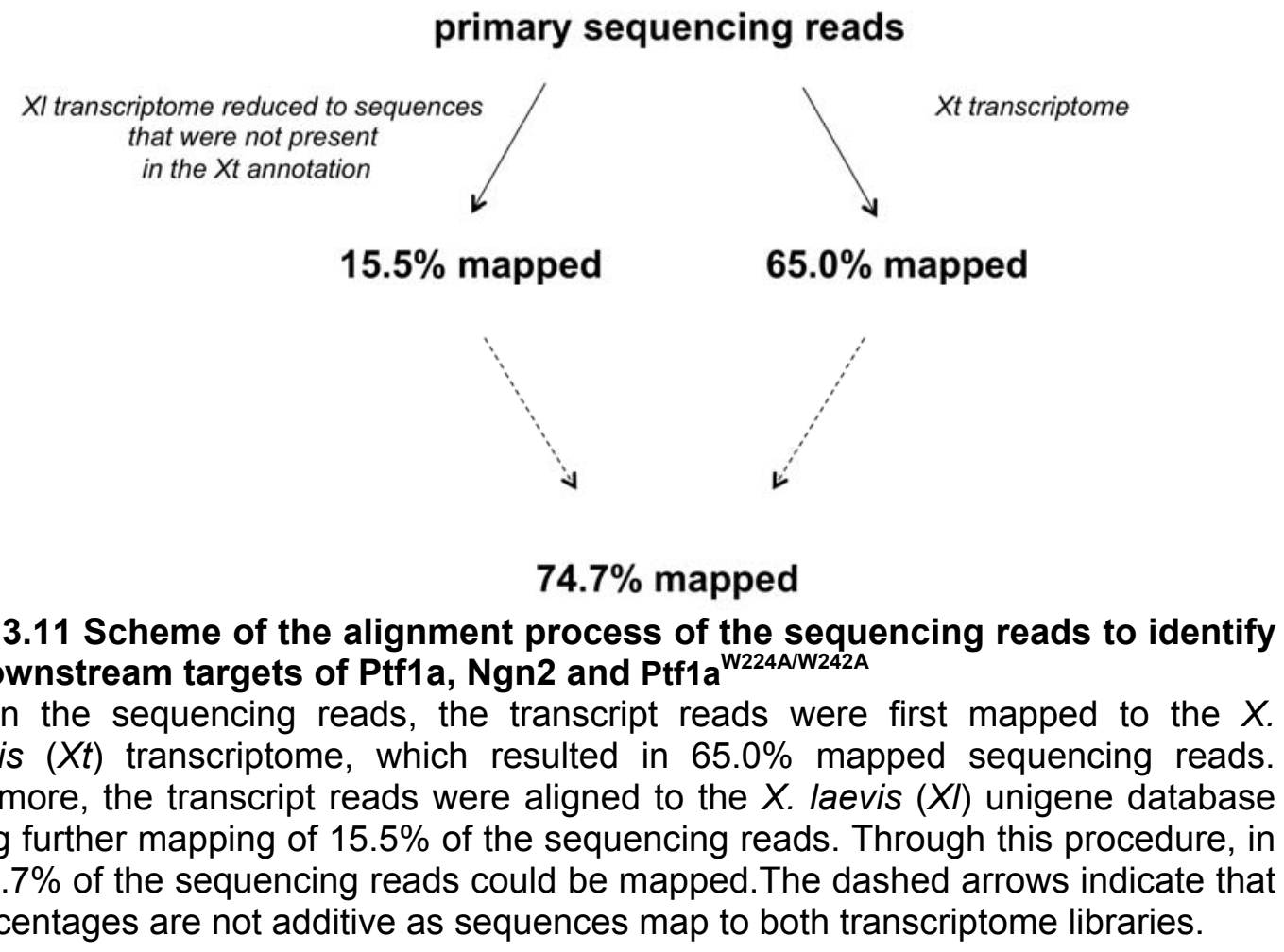

Applying a minimum two-fold change compared to control caps, Ptf1a-

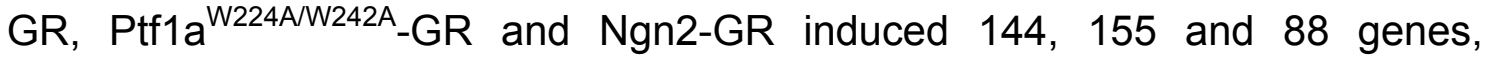
respectively after $6 \mathrm{~h}$ of protein induction (Fig. 3.12 B). Increasing this threshold revealed that a significant number of genes was activated more than 10-fold, but not over 50-fold (Fig. 3.12 B).

As expected, after $25 \mathrm{~h}$ of protein induction, the number of induced genes was increased. Ptf1a-GR, Ptf1 ${ }^{\text {W224A/W242A }}{ }^{-G R}$ and Ngn2-GR induced 1636, 369 and 762 genes, respectively, by more than two-fold (Fig. 3.12 C). In contrast to the $6 \mathrm{~h}$ time point, application of different thresholds indicated that a high number of genes was still upregulated more than 50 -fold and even more than 100-fold (Fig. 3.12 C). 
A Ptf1a-GR $(20 \mathrm{pg})$

Ptf1aW224A/W242A-GR (20 pg)

Ngn2-GR (20 pg)
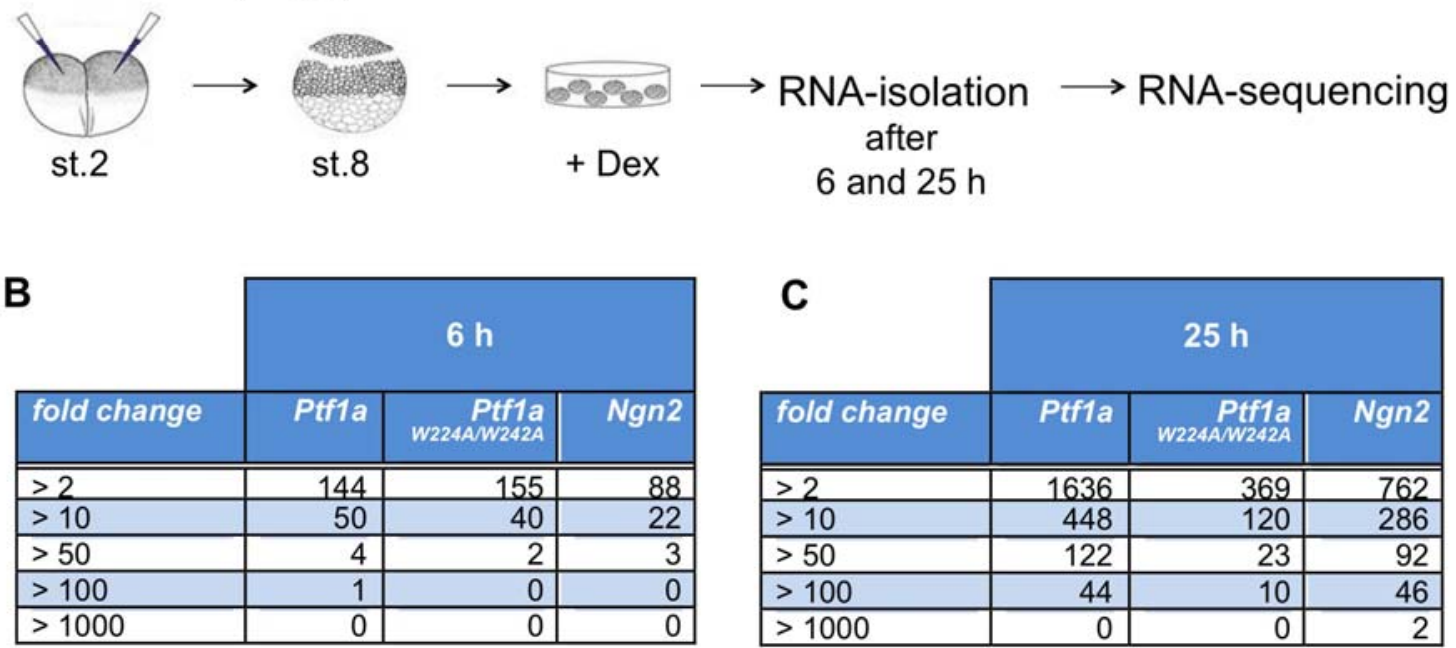

Figure 3.12 Temporal expression analysis of Ptf1a-GR, Ngn2-GR and Ptf1a ${ }^{\text {W224A/W242A }}$-GR target genes by whole transcriptome RNA-sequencing.

(A) The indicated mRNAs (20 pg each) were injected into both blastomeres of two-cell stage embryos, animal caps excised at blastula stage and treated with dexamethasone for 6 and $25 \mathrm{~h}$. Total RNA was isolated and subjected to RNA-sequencing. (B-C) Summary of identified target genes of Ptf1a, Ngn2 and Ptf1 $a^{\text {W224A/W242A }}$ after 6 h (B) and $25 \mathrm{~h}(\mathrm{C})$ after application of different fold-change categories. Given is the number of target genes with a two-, 10-, 50-, 100- or 1000-fold change compared to uninjected control caps (CC).

\subsection{Analysis of identified downstream targets of Ptf1a, Ngn2 and Ptf1 a ${ }^{\text {W224A/W242A }}$}

The two-fold cutoff was chosen for the more detailed analysis of the

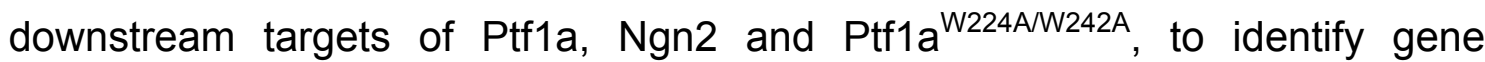
functions that are regulated by Ptf1a and Ptf1a ${ }^{\text {W224A/W242A }}$. The analysis revealed that a similar number of genes were induced by Ptf1a-GR (144 genes) and Ptf1a ${ }^{\text {W224A/W242A }}$ GR (155 genes) after $6 \mathrm{~h}$ of dexamethasone induction (Table 3.1), while for Ngn2-GR the number was much lower (88 genes) (Table 3.1). A total of 48 genes were shared between Ptf1a-GR, Ngn2-GR and Ptf1a ${ }^{\text {W224AW242A }}-G R$, which represents more than one third of the genes identified for Ptf1a-GR and Ptf1a ${ }^{\text {W224A/W242A }}$-GR (33\% and 31\%, respectively) and more than the half of the genes downstream of Ngn2-GR (55\%) (Table 3.1).

The number of genes induced by Ptf1a-GR strongly increased over time, with 1636 genes being identified after $25 \mathrm{~h}$ (Table 3.1). However, for Ptf1a ${ }^{\text {W224A/W242A }}-G R$, which induced at the early time point approximately the 
same number of genes compared to the wild-type Ptf1a-GR, only 369 genes were activated after $25 \mathrm{~h}$ (Table 3.1). The number of genes for Ngn2-GR was also lower, with 762 genes being identified (Table 3.1). A total of 276 genes were shared between the three transcription factors, which comprised more than $70 \%$ of total genes induced by Ptf1a $a^{\text {W224A/W242A }}-\mathrm{GR}$, but only $17 \%$ of the genes downstream of Ptf1a-GR (Table 3.1).

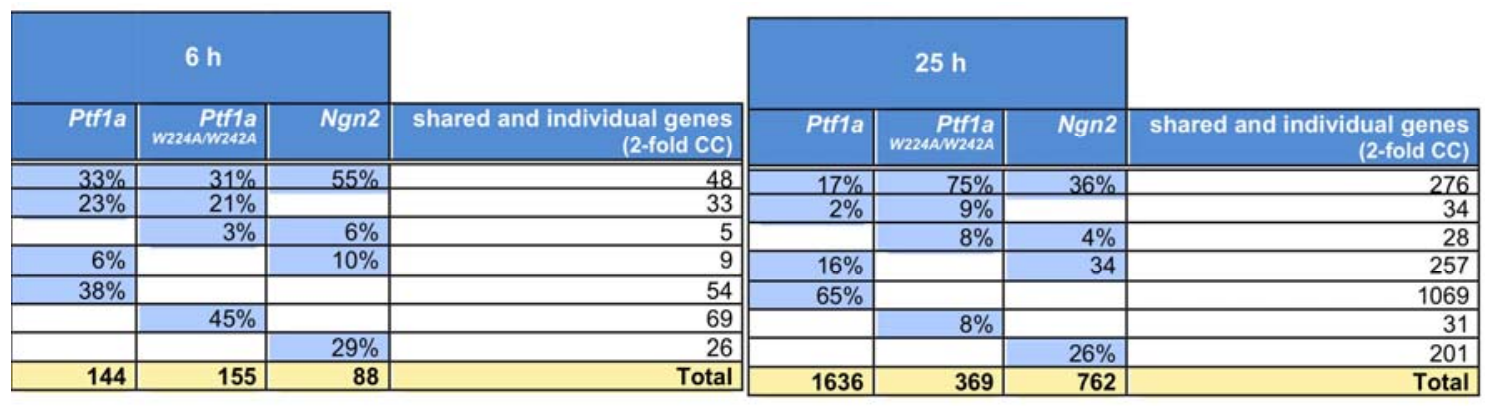

Table 3.1 Comparison of the number of genes that were induced by Ptf1a-GR, Ngn2-GR and Ptf1a ${ }^{\text {W224A/W242A }}$-GR after 6 and 25 h.

Represented is the number of shared and unique target genes of Ptf1a-GR, Ngn2-GR and Ptf1a ${ }^{\text {W224A/W242A }}-G R$ (right side) and the percentage of these compared to the total number of genes (yellow) identified with a two-fold or more increase compared to uninjected control caps (CC) for each transcription factor.

To gain functional insight into the genes identified in the screen, gene ontology was performed using DAVID (Database for Annotation, Visualization and Integrated Discovery, http://david.abcc.ncifcrf.gov/). In a first step, the

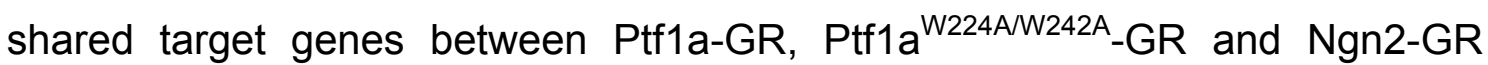
were investigated. Most of the 48 downstream target genes shared after $6 \mathrm{~h}$ of protein induction were significantly involved in transcriptional regulation and regulation of RNA metabolic processes (Fig. 3.13 A). A more detailed analysis showed that 16 genes present in this group have been previously associated with general neurogenesis in $X$. laevis, including neuronal differentiation (Ebf2, MyT1), lateral inhibition (DII1, Notch1) and cell cycle exit (Cdknx) (Dubois et al., 1998; Coffman et al., 1990; Bellefroid et al., 1996; Chitnis et al., 1995; Vernon et al., 2003).

Gene ontology analysis of the 276 shared target genes after $25 \mathrm{~h}$ showed significant enrichment of multiple processes and several could be associated with general neuron development such as axogenesis, cell morphogenesis involved in neuron differentiation, cell motion and transmission of nerve impulse (Fig. $3.13 \mathrm{~B}$ ). However, the majority of the common target 
genes are involved in cell adhesion, neuron differentiation and neuron projection (Fig. $3.13 \mathrm{~B}$ ). In summary, the group of shared target genes by Ptf1aGR, Ngn2-GR and Ptf1a ${ }^{\text {W224A/W242A }}-$ GR is suggested to reflect the general ability of proneural bHLH transcription factors to drive neurogenesis.

A

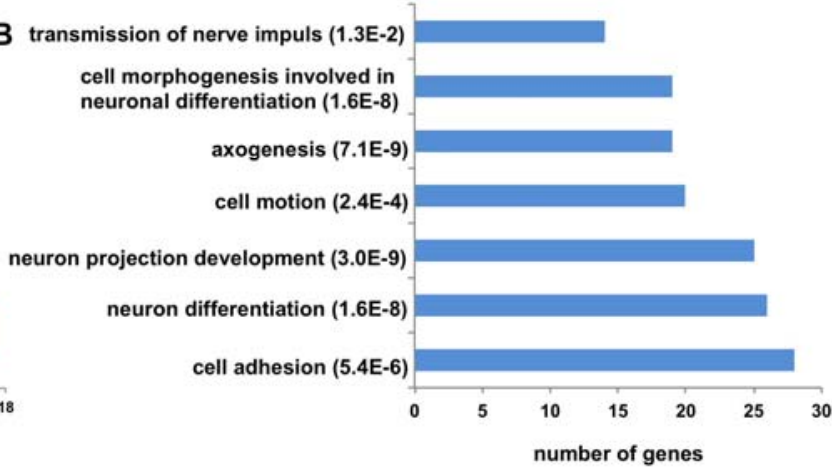

Figure 3.13 Overview of the biological processes enriched in the shared target genes by Ptf1a, Ptf1a ${ }^{\text {W224A/W242A }}$ and Ngn2 after 6 h (A) and $25 \mathrm{~h}$ (B).

The number of genes in each category is shown as well as the $P$ value at false discovery rate.

To identify the downstream network specific for the two distinct Ptf1a transcription complexes, the sets of induced candidates by Ptf1a-GR and Ptf1a ${ }^{\text {W224A/W242A }-G R}$ were compared. The analysis revealed that already $6 \mathrm{~h}$ after dexamethasone induction, Ptf1a-GR induced 54 target genes (38\% of total) that were not activated by Ptf1a $\mathrm{a}^{\text {W224A/W242A }}-\mathrm{GR}$ (Table 3.1), suggesting that some $\mathrm{Su}(\mathrm{H})$-dependent target genes are activated early. In order to gain insight into the function of the early $\mathrm{Su}(\mathrm{H})$-dependent target genes, gene ontology analysis was performed. This analysis associated the identified genes with tissue and embryonic morphogenesis as well as neural tube closure (Fig. 3.14 A). However, the majority of the identified early $\mathrm{Su}(\mathrm{H})$-dependent candidates are involved in transcription. Congruent to the Nanostring analyses (Fig. 3.2 and Fig. 3.8), none of the known genes involved in the later phase of GABAergic neuronal subtype specification such as $P a x 2, L b \times 1$ and $L h \times 1 / 5$ were among the early $\mathrm{Su}(\mathrm{H})$-dependent candidate genes.

The number of $\mathrm{Su}(\mathrm{H})$-dependent target genes highly increased at the 25 h time point, with Ptf1a-GR activating 1069 unique genes (65\% of total) that were not activated by Ptf1a ${ }^{\text {W224A/W242A }}-$ GR (Table 3.1). The list of the late Su(H)dependent Ptf1a target genes is associated with many different biological activities that are not only associated with the development of the nervous 
system. The majority of the $\mathrm{Su}(\mathrm{H})$-dependent Ptf1a target genes could be linked to transcriptional regulation, regulation of RNA metabolic processes and cell cycle regulation (Fig. 3.14 B). However, further processes were highly enriched in this data set such as chromosome organization, neuron differentiation, regulation of cell proliferation and DNA metabolic process (Fig. 3.14 B). In terms of molecular function, the $\mathrm{Su}(\mathrm{H})$-dependent target genes had not only a function in DNA binding (161 genes, $P$ value $=1.6 \mathrm{E}-11$ ), but also in binding chromatin (27 genes, $P$ value $=3.0 \mathrm{E}-8)$ and calcium ions $(56$ genes, $P$ value $=7.1 \mathrm{E}-2)$. $A$ more detailed analysis showed that consistent with the previous Nanostring data, the transcription factors involved in GABAergic interneuronal subtype specification $L b x 1, L h \times 1 / 5$ and $P a x 2$ as well as Gad1 were among the late $\mathrm{Su}(\mathrm{H})$-dependent Ptf1a-GR target genes.
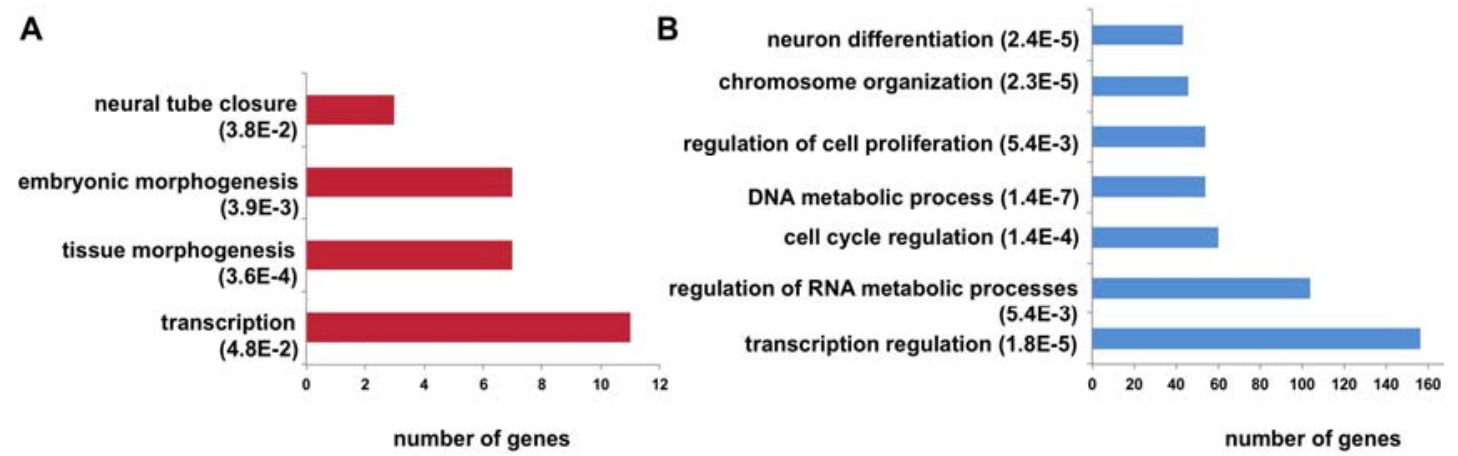

Figure 3.14 Overview of the biological processes enriched in the $\mathrm{Su}(\mathrm{H})$ dependent Ptf1a target genes after $6 \mathrm{~h} \mathrm{(A)}$ and $25 \mathrm{~h}$ (B).

The number of genes in each category is shown as well as the $P$ value at false discovery rate.

Interestingly, Ptf1a $\mathrm{a}^{\text {W224A/W242A }}-\mathrm{GR}$ mRNA overexpression induced 69 unique genes ( $45 \%$ of total) after $6 \mathrm{~h}$ and 31 ( $8 \%$ of total) after $25 \mathrm{~h}$ (Table 3.1). This suggests that especially early, Ptf1a ${ }^{\text {W224A/W242A }-G R}$ does not simply mimic the activity of Ngn2 as would have been suggested by the Nanostring analysis using known genes involved in this process. However, gene ontology analysis revealed no significant enrichment of any biological processes. While most of the genes activated by Ptf1a ${ }^{\text {W224A/W242A }}-$ GR and Ngn2-GR were also induced by Ptf1a-GR, in total 28 genes were not activated by wild-type Ptf1a-GR (Table 3.1). Included in these target genes was $T / x 3$, which is a marker of glutamatergic excitatory neurons. 


\subsection{The majority of genes induced after 6 and $25 \mathrm{~h}$ by Ptf1a,} Ngn2 and Ptf1 a ${ }^{\text {W224A/W242A }}$ are not shared

Comparison of the genes induced by Ngn2-GR, Ptf1a-GR and Ptf1a ${ }^{\text {W224AWW242A }-G R}$ after 6 and $25 \mathrm{~h}$, reveals that most transcripts are not shared between these two time points (Fig. 3.15). While many of the genes induced after $25 \mathrm{~h}$ are most likely indirect downstream targets of Ptf1a-GR,

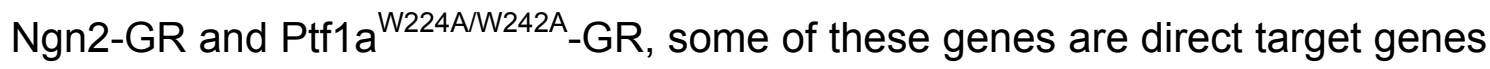
representing the later neuronal subtype inducing-activity such as Kirre/2 for Ptf1a.
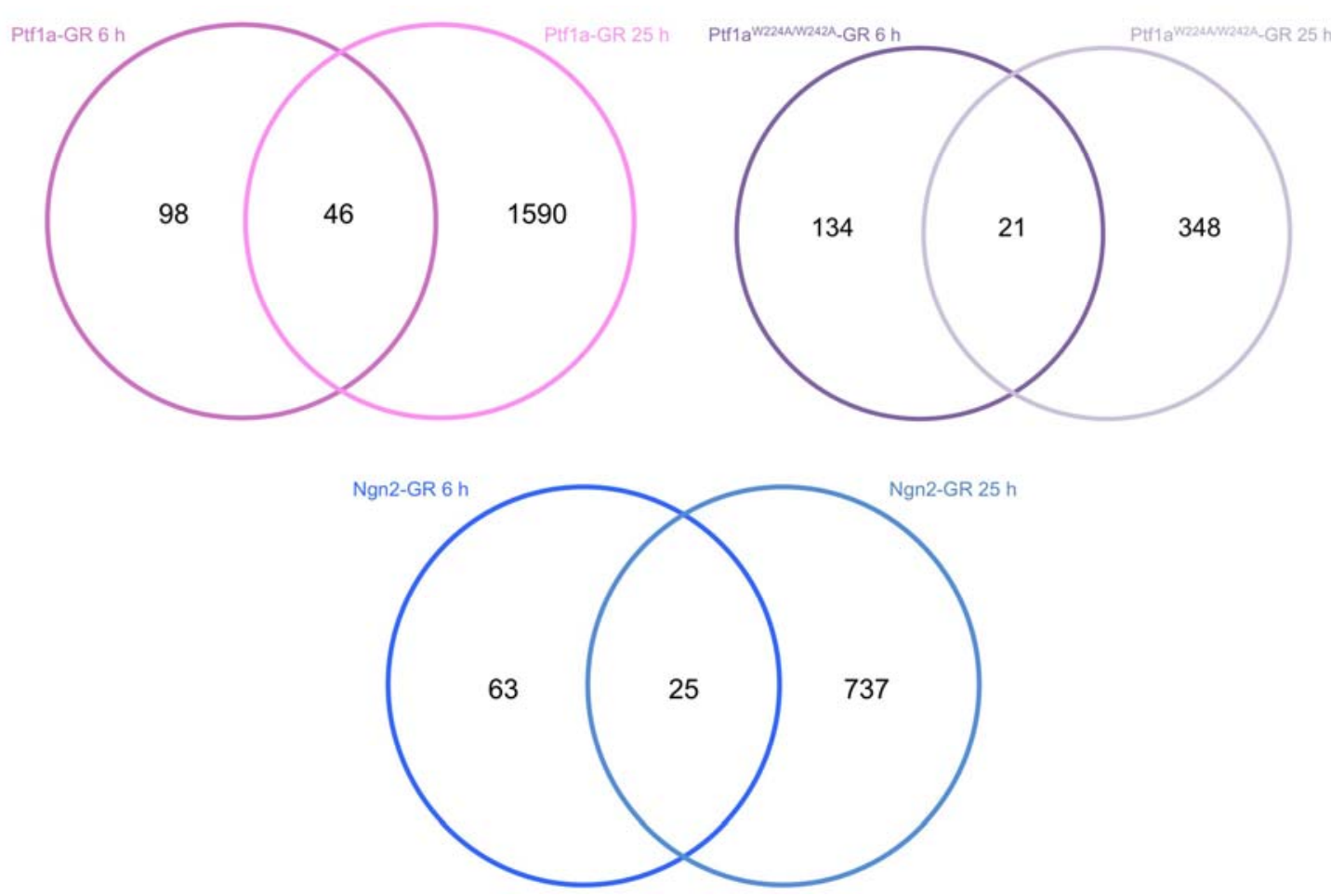

Figure 3.15 Distinct transcripts are present after 6 and 25 h of Ptf1a-GR, Ngn2-GR and Ptf1a ${ }^{\text {W224A/W242A }}$-GR induction.

Venn diagrams show unique and shared target genes at 6 and $25 \mathrm{~h}$ for Ptf1a-GR, Ngn2-GR and Ptf1a ${ }^{\text {W224A/W242A }}-$ GR, respectively.

\subsection{Inhibition of protein synthesis allows earlier activation of} Ngn2 expression

It is rather surprising that known murine direct Ptf1a Su(H)-dependent target genes such as Ngn2 and Kirrel2 are activated only after $25 \mathrm{~h}$, but not after $6 \mathrm{~h}$ as it would be anticipated. In fact, as shown by Nanostring (Fig. 3.2 and Fig. 3.8) and RNA-sequencing (Table 3.1) analyses, most of the $\mathrm{Su}(\mathrm{H})$ dependent genes are activated later than the $\mathrm{Su}(\mathrm{H})$-independent genes. There 
are many mechanisms that may explain this result. For example, the dimeric Ptf1a complex may activate expression of factors that repress expression of the $\mathrm{Su}(\mathrm{H})$-dependent target genes.

To determine, if Ptf1a directly activates Ngn2, its induction by Ptf1a and $\mathrm{Su}(\mathrm{H})$ was analysed in the presence of the protein synthesis inhibitor cycloheximide (CHX). Ptf1a-GR or Su(H)-GR mRNA were injected alone or in combination into two-cell stage embryos, animal caps excised and treated for 1 $\mathrm{h}$ with or without $\mathrm{CHX}$ to block translation (Fig. 3.16 A). Afterwards, the animal caps were cultivated for $8.5 \mathrm{~h}$ in the presence or absence of $\mathrm{CHX}$ and dexamethasone (Fig. 3.16 A). As a control, injected and control caps were treated only with $\mathrm{CHX}$ and did not express Ngn2. Consistent with previous results (Fig. 3.10), microinjection of Ptf1a-GR mRNA together with $S u(H)-G R$ mRNA, but not of either one alone, led to a strong induction of Ngn2 (Fig. 3.16 B). However, in the presence of CHX, Ptf1a-GR mRNA alone was sufficient to induce Ngn2 to a level similar to when Ptf1a-GR and Su(H)-GR mRNA were coinjected in the absence of $\mathrm{CHX}$ (Fig. 3.16 B). The level of $\mathrm{Ngn} 2$ induction through co-expression of Ptf1a-GR and Su(H)-GR mRNAs was increased in the presence of $\mathrm{CHX}$ (Fig. $3.16 \mathrm{~B}$ ). This finding is compatible with the idea that a mechanism, which is dependent on protein synthesis, prevents the early activation of the late $\mathrm{Su}(\mathrm{H})$-dependent target genes. 
A Ptf1a-GR $(20 \mathrm{pg})$

$\mathrm{Su}(\mathrm{H})-\mathrm{GR}(40 \mathrm{pg})$
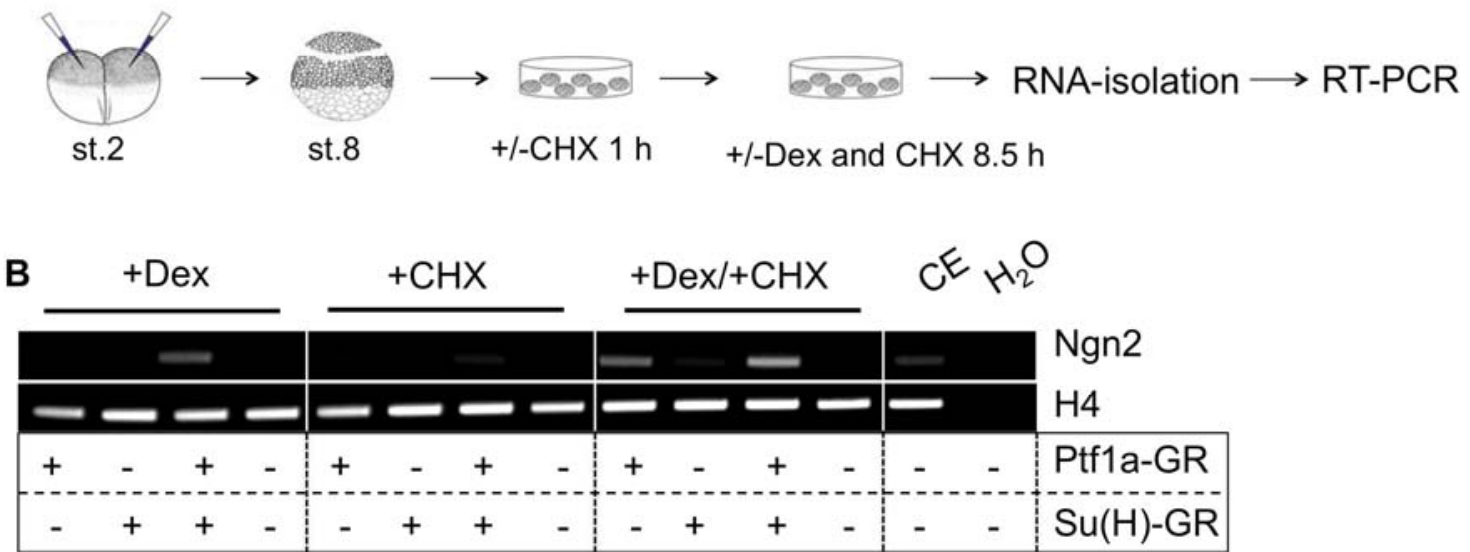

Figure 3.16 Inhibition of protein synthesis allows earlier activation of Ngn2 expression.

Comparative analysis of the activity of Ptf1a-GR alone and together with $\mathrm{Su}(\mathrm{H})-\mathrm{GR}$ in the presence of cycloheximide (CHX). (A) Ptf1a-GR and Su(H)-GR mRNA were injected alone or in combination into two-cell stage embryos. Animal caps were excised at the blastula stage and treated with our without $\mathrm{CHX}$ for $1 \mathrm{~h}$ to block protein synthesis. Afterwards, the animal caps were treated with or without dexamethasone (Dex)/CHX to induce protein activity. (B) Gene expression was analysed by semiquantitative RT-PCR. Expression levels were shown by $\mathrm{H} 4$ expression. CE, control embryos

\subsection{Identification of direct Ptf1a target genes}

In the previous RNA-sequencing experiment (Fig. 3.12), Ptf1a-GR mRNA induced more than 100 genes (two-fold or more) after $6 \mathrm{~h}$. Most likely not all of these early-induced genes are direct targets of Ptf1a. Moreover, it could be shown that Ptf1a Su(H)-dependent genes, such as Ngn2, are delayed in their activation, but either co-injection with $\mathrm{Su}(H)$ or treatment with $\mathrm{CHX}$ allows early activation (Fig. 3.16). On the basis of these findings, RNA-sequencing analysis was performed with the aim of identifying both, $\mathrm{Su}(\mathrm{H})$-dependent and independent Ptf1a direct target genes.

Ptf1a-GR and Su(H)-GR mRNA alone or in combination were microinjected into both blastomeres of two-cell stage embryos and animal caps were isolated from control and injected embryos (Fig. 3.18 A). After one hour of treatment with $\mathrm{CHX}$, the animal caps were cultivated for additional $8.5 \mathrm{~h}$ in dexamethasone and $\mathrm{CHX}$ (Fig. 3.18 A). After total RNA isolation, the samples were subjected to RNA-sequencing and sequences mapped as described previously (section 3.11, Fig. 3.11). In total, $67.3 \%$ of the sequencing reads could be mapped (Fig. 3.17). 


\section{primary sequencing reads}

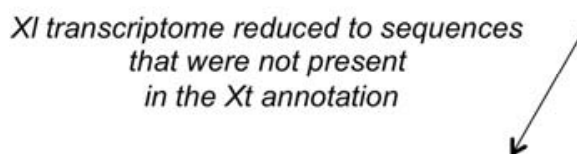

$16.3 \%$ mapped

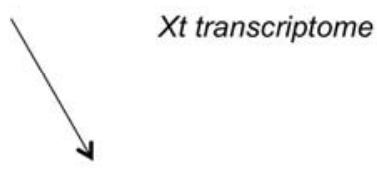

$56.2 \%$ mapped

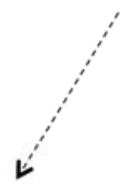

\section{$67.3 \%$ mapped}

Figure 3.17 Scheme of the alignment process of the sequencing reads to identify new direct downstream targets of Ptf1a.

To align the sequencing reads, the transcript reads were first mapped to the $X$. tropicalis $(X t)$ transcriptome, which resulted in $56.2 \%$ mapped sequencing reads. Furthermore, the transcript reads were aligned to the $X$. laevis $(X I)$ unigene database allowing further mapping of $16.3 \%$ of the sequencing reads. Through this procedure, in total $67.3 \%$ of the sequencing reads could be mapped. The dashed arrows indicate that the percentages are not additive as sequences map to both transcriptome libraries.

In animal caps injected with Ptf1a-GR mRNA alone or together with Su(H)-GR mRNA, 258 and 638 genes, respectively, were activated more than two-fold compared to uninjected control caps (Fig. 3.18 B). In contrast, only two genes were induced in animal caps by $S u(H)-G R$ alone. Different thresholds were applied to investigate the strength of gene induction. By applying a minimum fold change of $10, \mathrm{Su}(\mathrm{H})-\mathrm{GR}$ alone did not induce any genes, while Ptf1a-GR and Ptf1a-GR together with Su(H)-GR activated 87 and 222 genes, respectively (Fig. $3.18 \mathrm{~B}$ ). Increasing the cutoff to a 50 fold-activation, stronlgy decreased the number of candidate genes for Ptf1a-GR alone but not for Ptf1aGR together with $\mathrm{Su}(\mathrm{H})-\mathrm{GR}$. 


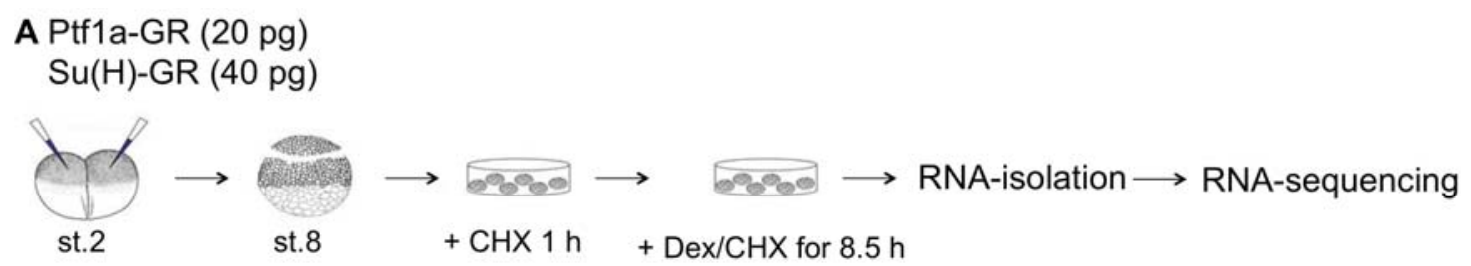

\begin{tabular}{|l|r|r|r|}
\cline { 2 - 4 } \multicolumn{1}{c|}{} & \multicolumn{3}{c|}{ CHX } \\
\hline B & \multicolumn{3}{c|}{} \\
\hline \hline$>2$ & 258 & 638 & 2 \\
\hline$>10$ & 87 & 222 & 0 \\
\hline$>50$ & 15 & 65 & 0 \\
\hline$>100$ & 9 & 19 & 0 \\
\hline$>1000$ & 0 & 2 & 0 \\
\hline
\end{tabular}

Figure 3.18 Identification of direct target genes induced by Ptf1a-GR and Su(H)GR alone and in combination by whole transcriptome RNA-sequencing.

(A) The indicated mRNAs were injected alone or together into both blastomeres of twocell stage embryos, animal caps excised at blastula stage and treated with $\mathrm{CHX}$ for $1 \mathrm{~h}$ to block protein synthesis. Afterwards, the animal caps were treated with dexamethasone/CHX for $8.5 \mathrm{~h}$ to induce protein activity. Total RNA was isolated and subjected to RNA-sequencing. (B) Summary of identified direct target genes of Ptf1a and $\mathrm{Su}(\mathrm{H})$ alone and in combination after application of different fold-change categories. Given is the number of target genes with a two-, 10-, 50-, 100 or 1000-fold change compared to uninjected control caps (CC).

\subsection{Identification of direct target genes induced in the previous temporal expression analysis by RNA-sequencing}

First, it was determined, which of the Ptf1a candidate genes induced in the earlier RNA-sequencing experiment analysing the temporal expression of Ptf1a target genes (Fig. 3.12), are indeed direct Ptf1a target genes. From the 144 candidate genes that were activated after 6 h, 86 genes $(60 \%)$ were also induced by Ptf1a-GR in the presence of $\mathrm{CHX}$ (Table 3.2). In contrast, from the 1636 target genes that were induced after $25 \mathrm{~h}$, only 63 genes (4\%) were induced by Ptf1a-GR in the presence of CHX (Table 3.2). When Ptf1a-GR and $\mathrm{Su}(H)-G R$ mRNA were co-injected, the number of candidate genes increased compared to Ptf1a-GR mRNA alone. From the 144 candidate genes induced after $6 \mathrm{~h}, 106$ genes (74\%) were identified as direct targets (Table 3.2), while from the 1636 genes activated at the late time point, 171 genes (10\%) were identified (Table 3.2). 


\begin{tabular}{|c|c|c|c|c|}
\hline \multicolumn{4}{|c|}{$\begin{array}{c}\text { Identification of direct Ptf1a targets activated } \\
\text { after } 6 \text { and } 25 \mathrm{~h}\end{array}$} & \multirow[b]{2}{*}{$\begin{array}{r}\text { shared genes } \\
(2 \text {-fold CC) } \\
\end{array}$} \\
\hline $\begin{array}{r}\text { Ptfla } \\
6 \mathrm{~h} \\
\end{array}$ & $\begin{array}{r}\text { Ptf1a } \\
25 \mathrm{~h} \\
\end{array}$ & $\begin{array}{r}\text { Ptf1a } \\
\text { CHX } \\
\end{array}$ & $\begin{array}{r}\text { Ptf1a+Su(H) } \\
\mathrm{CHX} \\
\end{array}$ & \\
\hline \multirow[t]{2}{*}{$60 \%$} & & $33 \%$ & & 86 \\
\hline & $4 \%$ & $24 \%$ & & 63 \\
\hline \multirow[t]{2}{*}{$74 \%$} & & & $17 \%$ & 106 \\
\hline & $10 \%$ & & $27 \%$ & 171 \\
\hline 144 & 1636 & 258 & 638 & Total \\
\hline
\end{tabular}

Table 3.2 Identification of direct Ptf1a targets activated after $\mathbf{6}$ and $25 \mathrm{~h}$.

Comparison of the identified genes of the two-independent transcriptome RNAsequencing approaches to identify bona fide Ptf1a downstream targets during $X$. laevis neurogenesis. Represented is the number of genes that are activated by Ptf1a-GR after 6 and $25 \mathrm{~h}$ and by Ptf1a-GR and Ptf1a-GR together with $\mathrm{Su}(\mathrm{H})$ in the presence of $\mathrm{CHX}$ as well as the percentage of these compared to the total number of genes (yellow) identified with a two-fold or more increase compared to uninjected control caps (CC) for each condition alone.

To identify the biological processes enriched in the direct target genes of Ptf1a-GR during the time course, gene ontology analysis was performed using DAVID for genes activated by Ptf1a-GR in combination with $\mathrm{Su}(\mathrm{H})-\mathrm{GR}$. At the 6 $\mathrm{h}$ time point, most of the 106 candidates were linked to transcriptional regulation and regulation of RNA metabolic processes (Fig. 3.19 A). Further biological processes significantly enriched in this data set were e.g. cell fate commitment, neuron differentiation and Notch-signalling (Fig. 3.19 A). Similar, most of the 171 direct target genes at $25 \mathrm{~h}$ are involved in transcriptional regulation and regulation of RNA metabolic processes (Fig. 3.19 B). However, other processes were also present, including cell motion, cell projection organization, neuronal differentiation, cell adhesion and hindbrain development (Fig. 3.19 B). Interestingly, eye development as well as endocrine system development were also enriched (Fig. 3.19 B), reflecting two known developmental functions of Ptf1a during development. Interestingly, none of the transcription factors known to be involved in GABAergic interneuronal subtype determination such as Pax2 (Cheng et al., 2004), Lhx1/5 (Pillai et al., 2007) and Lbx1 (Cheng et al., 2005) were among the Ptf1a direct target genes, suggesting that their activation by Ptf1a is indirect. 
A

\section{B}

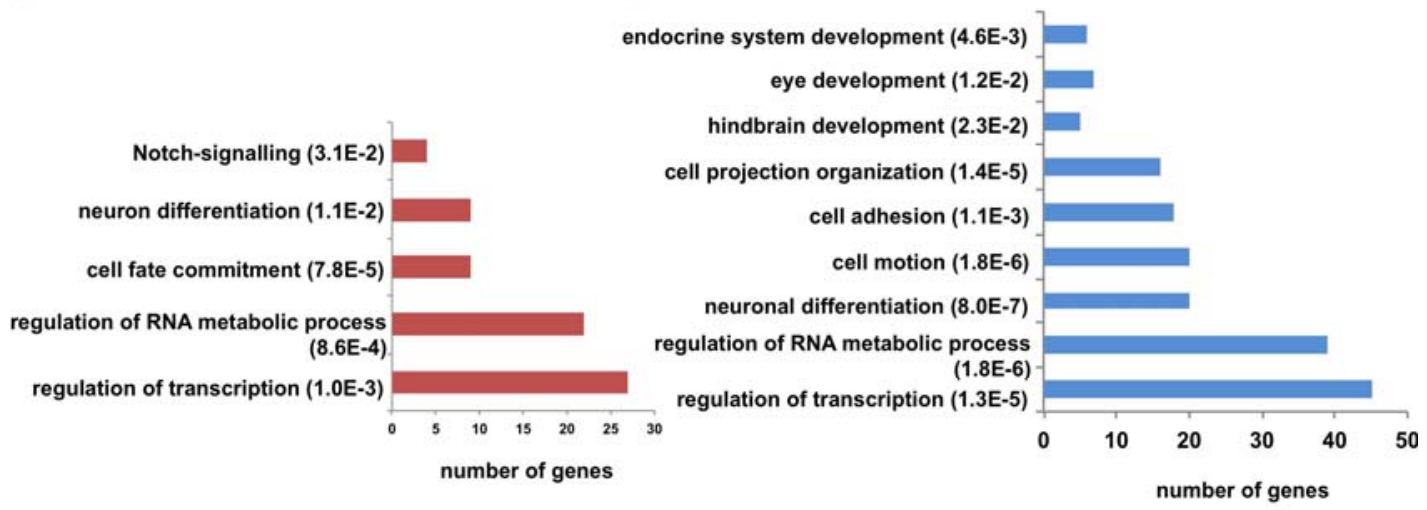

Figure 3.19 Enrichment of biological processes in $\mathrm{Ptf1} \mathrm{a} / \mathrm{Su}(\mathrm{H})$ direct target genes, which were also present in the time course analysis analysed by RNAsequencing after $6 \mathrm{~h}(\mathrm{~A})$ and $25 \mathrm{~h}(\mathrm{~B})$.

The number of genes in each category is shown as well as the $P$ value at false discovery rate.

\subsection{Analysis of direct targets of Ptf1a and Ptf1a/Su(H)}

The comparison of the two-independent RNA-sequencing experiments revealed that only a small number of the direct target genes were also present in the previous temporal expression analysis (Table 3.2). To analyse the total number of identified direct target genes, a minimal induction of two-fold was applied. Interestingly, Ptf1a-GR alone induced 64 genes (25\% of total) that were not activated by Ptf1a-GR in combination with $\mathrm{Su}(\mathrm{H})-\mathrm{GR}$ (Table 3.3). Gene ontology analysis revealed that translation (12 genes, $P$ value $=3.1 \mathrm{E}-15$ ) was the only biological process significantly enriched in this set of Ptf1a-GR unique target genes. In contrast, Ptf1a-GR together with $\mathrm{Su}(\mathrm{H})-\mathrm{GR}$ induced 443 unique target genes ( $70 \%$ of total) (Table 3.3 ). The biological processes enriched in this data set were similar to the analysis of Ptf1a-GR/Su(H)-GR targets that were also present in the temporal expression analysis after 6 and $25 \mathrm{~h}$ (Fig. $3.19 \mathrm{~B})$. One difference was that in the set of the total identified target genes, eye development was not significantly enriched anymore.

A more detailed analysis of the identified target genes revealed that also pancreas specific genes were induced in the presence of $\mathrm{CHX}$, however, only when Ptf1a-GR was overexpressed in combination with $S u(H)-G R$. Among these were Pdia2 and the digestive enzymes Cpa1 and Prss1. This is consistent with the finding that Ptf1a directly activates acinar digestive enzymes in the exocrine pancreas (Cockell et al., 1989). 


\begin{tabular}{|r|r|r|r|}
\hline \multicolumn{3}{|c|}{ CHX } & \\
\hline Ptf1a & Ptf1a+Su(H) & Su(H) & shared and individual genes \\
& & & (2-fold CC) \\
\hline \hline $0 \%$ & $0 \%$ & $50 \%$ & 1 \\
\hline $75 \%$ & $30 \%$ & & 193 \\
\hline $0 \%$ & $0 \%$ & $0 \%$ & 0 \\
\hline $25 \%$ & & $0 \%$ & 0 \\
\hline & & & 64 \\
\hline & $70 \%$ & & 443 \\
\hline 258 & & $50 \%$ & 1 \\
\hline
\end{tabular}

Table 3.3 Whole transcriptome RNA-sequencing to identify direct target genes of Ptf1a-GR and Ptf1a-GR together with Su(H)-GR.

Summary of identified direct target genes of Ptf1a, Su(H) and both together represented in a table. Given is the number of shared and unique target genes of Ptf1a-GR and $\mathrm{Su}(\mathrm{H})$-GR alone as well as in combination (right side) and the percentage of these compared to the total number of genes (yellow) identified with a two-fold or more increase compared to uninjected control caps (CC) for each transcription factor.

\subsection{Identification and validation of direct trimeric-dependent Ptf1a target genes}

Comparison of the two independent RNA-sequencing approaches allows identification of $\mathrm{Su}(\mathrm{H})$-dependent direct Ptf1a target genes. Genes that are

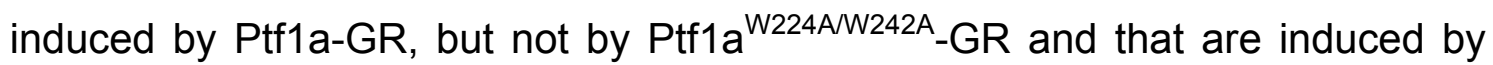
Ptf1a-GR or Ptf1a-GR/Su(H)-GR in the presence of $\mathrm{CHX}$ are expected to be trimeric-dependent Ptf1a direct target genes. Using this comparative approach, 111 putative direct trimeric-dependent transcripts were identified. Of these, 13 genes (12\%) were induced after $6 \mathrm{~h}$, and 34 genes (31\%) were induced without elevated $\mathrm{Su}(\mathrm{H})-\mathrm{GR}$ expression levels. To verify selective candidate genes, eight (Table 3.4) of the 111 identified genes as well as the known direct target gene Kirrel2 (Nishida et al., 2010) were analysed using the Nanostring nCounter RNA expression profiling system. 
Table 3.4. Summary of the fold-activation compared to control caps of the selected $\mathrm{Su}(\mathrm{H})$-dependent Ptf1a target genes.

\begin{tabular}{|l|r|r|r|r|}
\hline $\begin{array}{l}\text { Gene } \\
\text { Symbol }\end{array}$ & $\begin{array}{c}\text { Ptf1a-GR } \\
6 \mathrm{~h}\end{array}$ & $\begin{array}{c}\text { Ptf1a-GR } \\
25 \mathrm{~h}\end{array}$ & $\begin{array}{l}\text { Ptf1a-GR } \\
\text { CHX }\end{array}$ & $\begin{array}{r}\text { Ptf1a-GR +SuH-GR } \\
\text { CHX }\end{array}$ \\
\hline Prdm13 & 865.7 & 528.4 & 888.7 & 4650.1 \\
\hline Barh/2 & - & 26.9 & - & 108.5 \\
\hline Hmx3 & - & 2.4 & 53.4 & 84.8 \\
\hline Lhx2 & - & - & 58.1 & 63.7 \\
\hline Mecom & - & 9.6 & - & 12.6 \\
\hline Sox9 & - & 37.2 & - & 5.3 \\
\hline ESR10 & 5.3 & 4.0 & 6.0 & 3.1 \\
\hline FoxD3 & - & 56.2 & - & 2.6 \\
\hline
\end{tabular}

In a first approach, these genes were analysed for their temporal activation and their dependence on $\mathrm{Su}(\mathrm{H})$ interaction for gene induction in the animal cap assay (Fig. 3.20 and Appendix 6.1.3). Consistent with the results of the RNA-sequencing experiment, Prdm13 (Fig. 3.20 B) and Esr10 (Fig. 3.20 C) expression were activated by Ptf1a-GR after $6 \mathrm{~h}$ of dexamethasone induction, while the expression of the known direct target gene Kirre/2 (Fig. 3.20 D) as well as of Mecom (Fig. 3.20 E), Hmx3 (Fig. 3.20 F), FoxD3 (Fig. 3.20 G), Sox9 (Fig. $3.20 \mathrm{I}$ ) and Barh/2 (Fig. $3.20 \mathrm{H}$ ) were only activated after $25 \mathrm{~h}$. Microinjection of Ptf1a ${ }^{W 224 A W 242 A}-G R$ mRNA compared to Ptf1a-GR wild-type did not considerably induce the expression of Kirrel2 (Fig. 3.20 D), Prdm13 (Fig. 3.20 B), Hmx3 (Fig. 3.20 F), Mecom (Fig. 3.20 E), Barh/2 (Fig. 3.20 H) and FoxD3 (Fig. $3.20 \mathrm{G}$ ), demonstrating that their induction is strongly dependent on the interaction of Ptf1a with $\mathrm{Su}(\mathrm{H})$. Furthermore, Ptf1a ${ }^{W 224 A W 242 A}-G R$ was not able to induce the early expression of Esr10 (Fig. 3.20 C), while the late expression of this gene was not affected. Moreover, Ptf1a ${ }^{W 224 A / W 242 A}-G R$ mRNA overexpression was sufficient to induce the expression of Sox9 (Fig. 3.20 I), albeit compared to Ptf1a-GR the induction was reduced about the half. In summary, the interaction with $\mathrm{Su}(\mathrm{H})$ is required for strong induction of the novel identified $\mathrm{Su}(\mathrm{H})$-dependent direct Ptf1a target genes. 
A Ptf1a-GR (20 pg)

Ptf1aW224AW242A-GR (20 pg)

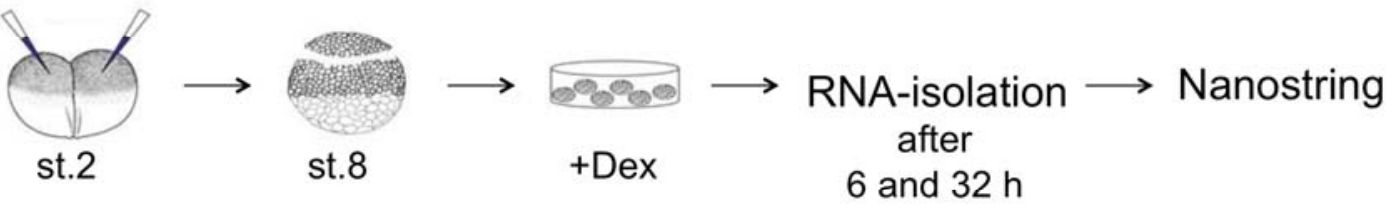

6 and $32 \mathrm{~h}$
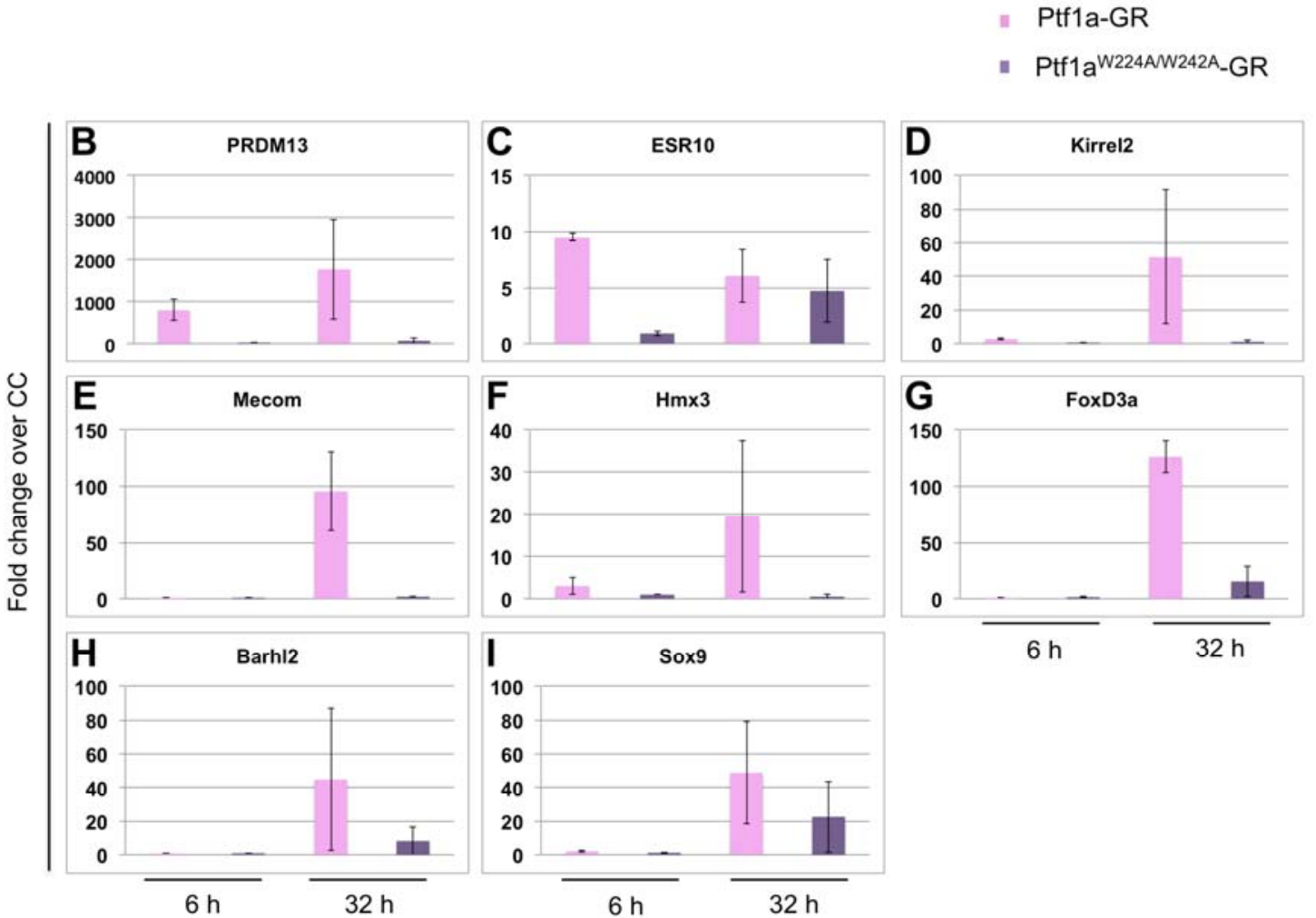

Figure 3.20 Verification of selected direct Ptf1a Su(H)-dependent target genes.

Comparative temporal analysis of wild-type Ptf1a and a mutant Ptf1a (Ptf1a ${ }^{\text {W224A/W242A }}$ ), containing two point mutations that disrupt the association with $\mathrm{Su}(\mathrm{H})$, to analyse, if the new identified Ptf1a target genes are dependent on $\mathrm{Su}(\mathrm{H})$ interaction. (A) The indicated mRNAs (20 pg each) were injected into both blastomeres of two-cell stage embryos, animal caps excised at the blastula stage and treated with dexamethasone (Dex) for 6 and $32 \mathrm{~h}$. Total RNA was isolated and marker gene expression analysed by Nanostring. (B-I) Shown is the averaged fold change over uninjected control caps (CC) of two independent experiments. Error bars represent the standard error of the mean (+/-SEM). Note the different scales in each diagram. Shown are graphs of selected genes, for a full list see Appendix 6.1.3.

To further verify that these genes are trimeric Ptf1a target genes, their gene induction in the presence of elevated $\mathrm{Su}(\mathrm{H})$ expression levels were investigated in animal cap assays (Fig. 3.21 and Appendix 6.1.4). Corresponding with the RNA-sequencing analysis, overexpression of $S u(H)-G R$ mRNA alone did not significantly increase the expression of any of the evaluated target genes. Ptf1a-GR activated after 3 and $6 \mathrm{~h}$ the expression of Prdm13 (Fig. $3.21 \mathrm{~B}$ ), consistent with the previous Nanostring analysis. Also 
congruent to the previous experiment, Ptf1a-GR activated Esr10 expression at the early time points (28-, 27-fold) (Fig. 3.21 C), however, the induction of Esr10 after $25 \mathrm{~h}$ (Fig. $3.21 \mathrm{C}$ ) was so strong that the early induction is not apparent on the graph. The expression of Kirrel2 (Fig. $3.21 \mathrm{D})$, Mecom (Fig. $3.21 \mathrm{~J}), \mathrm{Hm} \times 3$ (Fig. 3.21 E), FoxD3 (Fig. 3.21 F), Sox9 (Fig. $3.21 \mathrm{G}$ ) and Barh/2 (Fig. 3.21 H) was only activated by Ptf1a-GR after $25 \mathrm{~h}$ of dexamethasone induction, consistent with the previous analysis. Furthermore, Lhx2 (Fig. 3.21 I), a gene that was identified as a direct target of Ptf1a-GR and Ptf1a-GR/Su(H)-GR, but was surprisingly not present in the temporal gene expression analysis, was also only activated at the $25 \mathrm{~h}$ time point. Similar to what was observed for Ngn2 (Fig. $3.10 \mathrm{~L}$ ), co-expression of $S u(H)-G R$ with Ptf1a-GR mRNA led to an increase in gene induction of Prdm13 (Fig. $3.21 \mathrm{~B}$ ), Esr10 (Fig. $3.21 \mathrm{C}$ ), Kirrel2 (Fig. 3.21 D), FoxD3 (Fig. 3.21 F) and Sox9 (Fig. $3.21 \mathrm{G}$ ) as well as of Barh/2 (Fig. 3.21 H), Lhx2 (Fig. $3.21 \mathrm{I}$ ) and Mecom (Fig. $3.21 \mathrm{~J})$. The effect on the latter group of genes was particularly strong. In contrast, the expression of $\mathrm{Hm} \times 3$ (Fig. $3.21 \mathrm{E}$ ) was highly reduced, if not even abolished by co-expression of Ptf1a-GR and Su(H)-GR mRNA. Thus, similar to the previous analysis of $\mathrm{Su}(\mathrm{H})$ dependent target gene activation (Fig. 3.10), also the novel identified Ptf1a direct target genes respond differently to the co-expression of $\mathrm{Su}(\mathrm{H})-\mathrm{GR}$. 
A Ptf1a-GR $(20 \mathrm{pg})$

$\mathrm{Su}(\mathrm{H})-\mathrm{GR}(40 \mathrm{pg})$

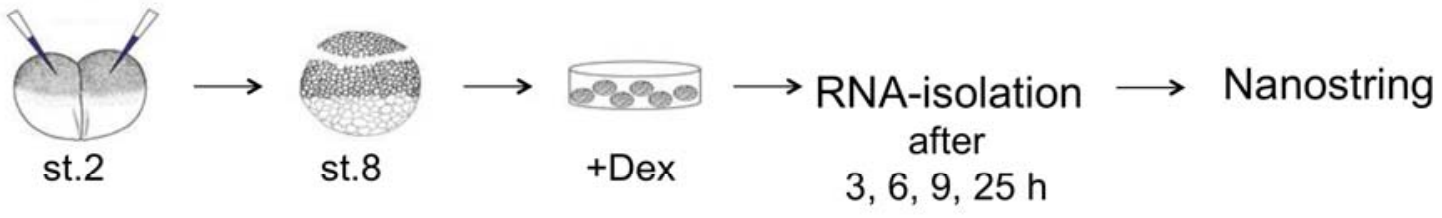

$3,6,9,25 \mathrm{~h}$
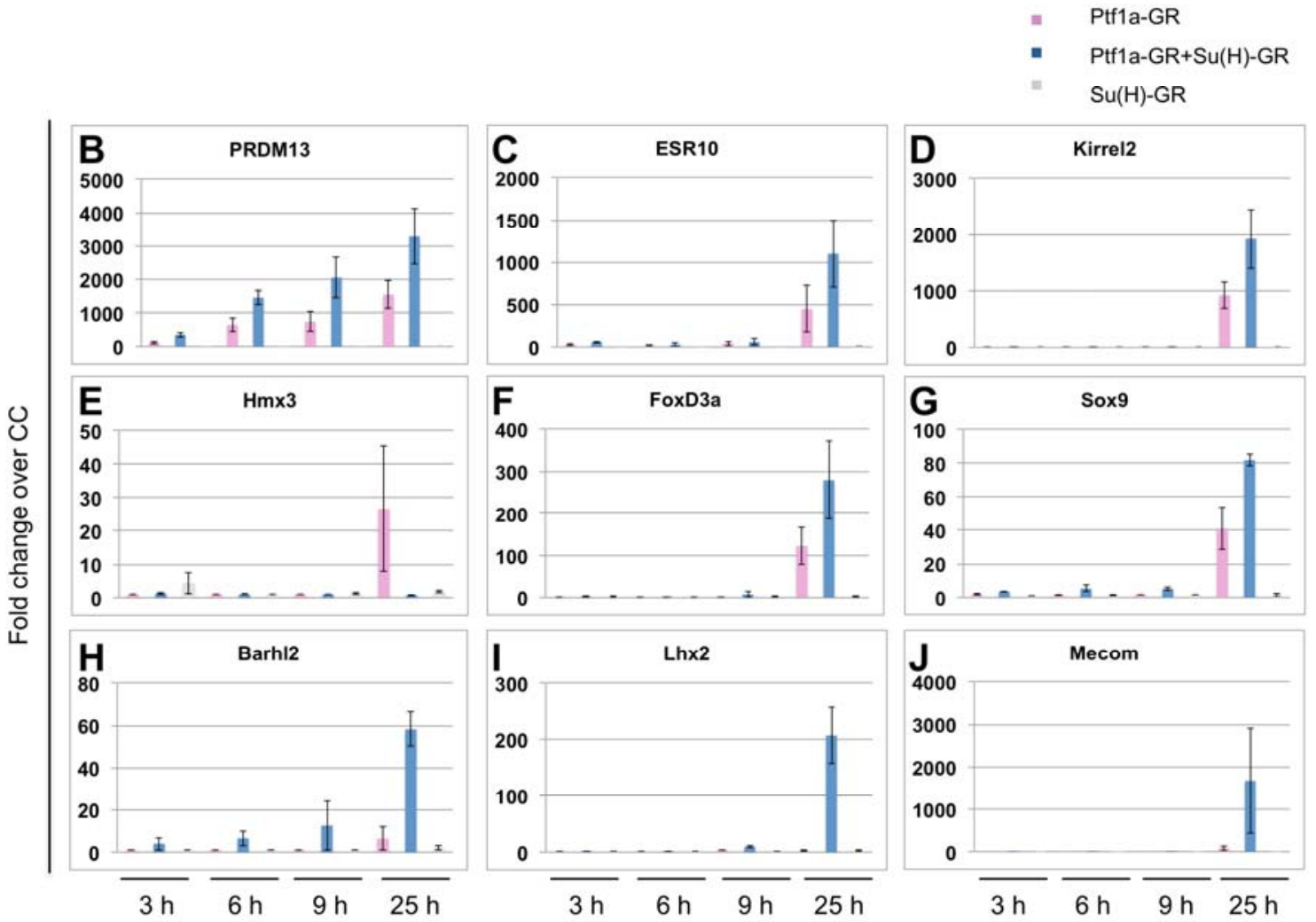

Figure 3.21 Co-expression of Ptf1a and $\mathrm{Su}(\mathrm{H})$ influences expression of new identified $\mathrm{Su}(\mathrm{H})$-dependent direct Ptf1a target genes.

Comparative analysis of the activity of Ptf1a-GR alone and together with $\mathrm{Su}(\mathrm{H})-\mathrm{GR}$. (A) Ptf1a-GR (20 pg) and Su(H)-GR (40 pg) were injected into both blastomeres of two-cell stage embryos, animal caps excised at the blastula stage and treated with dexamethasone (Dex). Animal caps were cultured for 3, 6, 9 or $25 \mathrm{~h}$, which corresponds to stage 9, 10, 10.5 and 23 of sibling embryos. RNA was isolated and gene expression analysed by Nanostring. (B-J) Shown is the averaged fold change over uninjected control caps (CC) of two independent experiments. Error bars represent the standard error of the mean (+/-SEM). Note the different scales in each diagram. Shown are graphs of selected genes, for a full list see Appendix 6.1.4. 


\section{Discussion}

\subsection{The proneural function of Ptf1a does not require interaction with Su(H)}

The bHLH transcription factor Ptf1a was shown to act as a selector gene for GABAergic inhibitory neurons at the expense of glutamatergic excitatory neurons in the retina, the dorsal horn of the spinal cord and in the cerebellum (Glasgow et al., 2005; Hori et al., 2008; Hoshino et al., 2005; Fujitani et al., 2006; Nakhai et al., 2007; Pascual et al., 2007; Dullin et al., 2007; Lelievre et al., 2011). Consistent with this, Ptf1a overexpression in $X$. laevis embryos and animal caps promoted not only Gad1 expression, but also induced the expression of Pax2, $L b \times 1$ and $L h \times 1 / 5$ at the expense of markers indicative of a glutamatergic excitatory neuronal identity (Fig. 3.1, Fig. 3.2 and Fig. 4.1). Furthermore, the use of a transcriptional activator Ptf1a (Ptf1a-VP16) provides evidence that the repression of the molecular markers indicative of glutamatergic neurons is indirect, probably due to the activation of a specific transcriptional repressor protein (Fig. 3.1).

Ptf1a was identified as a component of a heterotrimeric transcription factor complex, consisting of a commonly expressed class A bHLH transcription factor and Rbp-j/-I (Su(H)) (Beres et al., 2006). Detailed Nanostring analyses with a chimeric form of Ptf1a, which contained the bHLH domain of Ngn2, showed that the bHLH domain of Ptf1a is not essential for the determination of a GABAergic neuronal identity, even though the induction of genes involved in this process is reduced compared to wild-type Ptf1a (Fig. 3.7 and Fig. 4.1). Furthermore, the chimeric Ptf1a was still able to induce general neurogenesis and also marker genes indicative of glutamatergic excitatory neurons (Fig. 3.7 and Fig. 4.1). This suggests that the neuronal subtype inducing activity of Ngn2 is conferred through its bHLH domain and is consistent with experiments in $X$. laevis and Drosophila that implicated a role of the bHLH domain in target gene specificity (Talikka et al., 2002; Chien et al., 1996).

In $X$. laevis animal cap assays, a mutant version of Ptf1a $\left(P t f 1 a^{\text {W224A/W242A }}\right)$ that does not bind $\mathrm{Su}(\mathrm{H})$, is unable to promote the formation of GABAergic inhibitory neurons (Fig. 3.8 and Fig. 4.1); which is consistent with studies performed in the mouse and chick neural tube (Hori et al., 2008). 
Surprisingly, it was found that the general neurogenesis-inducing activity of Ptf1a ${ }^{\text {W224A/W242A }}$ was not impaired (Fig. 3.8), suggesting that the interaction with $\mathrm{Su}(\mathrm{H})$ is not required for the proneural function of Ptf1a. While Ptf1a was always considered to function in the context of a trimeric transcription factor complex, these findings suggest that Ptf1a forms a heterodimer with a general bHLH protein and only context-specifically additionally interacts with $\mathrm{Su}(\mathrm{H})$ as a cofactor. Through the formation of different transcription complexes, Ptf1a is able to promote distinct activities during the development of the nervous system: one complex drives general neurogenesis and another promotes neuronal subtype specification. This would be in line with the activity of other vertebrate proneural bHLH transcription factors that also play a role in neuronal subtype specification (Lee and Pfaff, 2003; Lo et al., 1998; Hatakeyama et al., 2001; Saba et al., 2005; Powell and Jarman, 2008; Guillemot, 2007; Brunet and Ghysen, 1999; Bertrand et al., 2002).

Through RNA-sequencing analysis, downstream target genes induced after 6 and $25 \mathrm{~h}$ by wild-type Ptf1a and Ptf1a ${ }^{\text {W224A/W242A }}$ were identified. In line with the finding that Ptf1a forms two distinct transcription complexes during general neurogenesis and neuronal subtype specification, both shared and distinct genes were induced by the wild-type and mutant Ptf1a. As the proneural activity of Ptfla was $\mathrm{Su}(\mathrm{H})$-independent, it was anticipated that the genes shared by both would be involved in general neurogenesis. In contrast, those that were induced by the wild-type Ptf1a but not by the mutant would be expected to be involved in neuronal subtype specification. This, indeed, was what was observed in the RNA-sequencing analysis. Genes such as Pax2, $L b \times 1$ and $L h \times 1 / L h \times 5$, indicative of GABAergic interneuronal subtype identity (Helms and Johnson, 2003), were only induced by wild-type Ptf1a after $25 \mathrm{~h}$. Additionally, the Ptf1a-trimeric complex also induced after $25 \mathrm{~h}$ the direct target genes Kirrel2 (Nishida et al., 2010) and Ngn2 (Henke et al., 2009b) as well as FoxD3 and Olig3, two genes with which Ptf1a promotes the formation of the glutamatergic climbing fiber neurons (Yamada et al., 2007; Storm et al. 2009). Moreover, among the genes shared between Ptf1a and the mutant Ptf1a were known genes involved in general neurogenesis. Correspondingly, the majority of the gene targets shared by the wild-type and mutant Ptf1a were also induced 
by Ngn2. This further supports the notion that this group of genes represents the proneural function of these three bHLH transcription factors.

A defect in general neurogenesis in mice lacking Ptf1a in the CNS has not been reported. Furthermore, lineage tracing experiments in a viable Ptf1a mutant mice, in which Ptf1a expression is only absent in the cerebellum but not in the pancreas, indicated that the cerebellar ventricular zone can produce neurons even in the absence of Ptf1a expression (Hoshino et al., 2005). However, redundancy between Ptf1a and other proneural bHLH transcription factors as it has been shown for Ngn2 and Ascl1 (Mash1) (Fode et al., 2000) was not excluded (Hoshino, 2006). In order to investigate a putative redundancy between Ptf1a and other bHLH transcription factors, it should be analysed, if other proneural transcription factors are upregulated in the cerebellum and dorsal horn of the spinal cord in Ptf1a knockout and mutant mice. While no other member of the Neurogenin neuronal determination gene family is expressed in the inner ventricular zone of the hindbrain that will give rise to GABAergic inhibitory neurons (Fig. 3.5), the proneural bHLH transcription factor Ascl1 is expressed in the mouse cerebellar ventricular zone similar to Ptf1a (Zordan et al., 2008) and might therefore compensate for the loss of Ptf1a proneural function. The strong proneural activity that Ptf1a exhibits in $X$. laevis embryos and explants, coupled with its expression in the progenitor cells suggests that Ptf1a may promote general neurogenesis also in other vertebrates.

\subsection{Ptf1 $\mathrm{a}^{\mathrm{W} 224 \mathrm{~A} / \mathrm{W} 242 \mathrm{~A}}$ is a functional transcription factor}

In animal cap assays evaluated by Nanostring analysis, Ptf1a ${ }^{\text {W224A/W242A }}$ was not only able to drive general neurogenesis, but also to induce genes indicative of glutamatergic excitatory neurons (Fig. 3.8 and Fig. 4.1). That Ptf1a bearing these mutations still promoted general neurogenesis and activated markers indicative of an alternative neuronal subtype was also reflected by RNA-sequencing. A small number of genes, including Hox11L2/TIx3, which is indicative of glutamatergic excitatory neurons, was both induced by Ptf1a W224A/W242A and Ngn2. Thus, glutamatergic excitatory neuronal subtype specification might be the 'default' activity of the heterodimeric Ptf1a, similar to Ngn2. A dramatic increase in glutamatergic excitatory neurons in the mouse 
neural tube is observed upon depletion of $\mathrm{Rbp}-\mathrm{j}(\mathrm{Su}(\mathrm{H}))$ in the presence of Ptf1a, demonstrating that Rbp-j, similar to Ptf1a, is required to promote GABAergic neurons at the expense of glutamatergic neurons (Hori et al., 2008). The requirement of the Ptf1a-SuH trimeric complex for GABAergic neuronal specification was further demonstrated using a single mutation to disrupt the binding of Ptf1a with $\mathrm{Su}(\mathrm{H})\left(\mathrm{Ptf} 1 \mathrm{a}^{\mathrm{W} 298 \mathrm{~A}}\right)$. Correspondingly, in homozygous Ptf1a ${ }^{\text {W298A }}$ mice, GABAergic inhibitory interneurons were lost, while the number of glutamatergic excitatory neurons was increased (Hori et al., 2008). As this phenotype is similar to that of Ptf1a knockout mice (Glasgow et al., 2005), it was concluded that the Ptf1a ${ }^{\text {W298A }}$ does not compensate for wild-type Ptf1a activity and is non-functional. However, it might be that the increase in glutamatergic neurons in Ptf1a ${ }^{\text {W298A }}$ mice was not only due to the loss of wildtype Ptf1a activity, but also that Ptf1a ${ }^{\text {W298A }}$ actively contributed to promote this neuronal subtype. To investigate this possibility, lineage tracing experiments in the Rbp-j deficient and Ptf1a ${ }^{\text {W298A }}$ mouse must be conducted to analyse the origin of these glutamatergic excitatory neurons. Moreover, RNA-sequencing identified genes induced by Ptf1a ${ }^{\text {W224A/W242A }}$ after 6 and $25 \mathrm{~h}$ that were neither activated by Ptf1a nor by Ngn2, indicating that Ptf1a W224A/W242A activates a unique set of target genes. This group of genes might reflect an additional unknown function of the Ptf1a mutant, however, gene ontology analysis revealed no specific biological processes enriched in these candidates. Taken together, the experiments performed in $X$. laevis suggest that in absence of $\mathrm{Su}(\mathrm{H})$ binding, Ptf1a is still a functional proneural bHLH transcription factor, which specifies glutamatergic excitatory neurons in the nervous system.

\begin{tabular}{|c|c|c|c|c|}
\hline neuronal subtype & Ptf1a-GR & Ngn2-GR & Ptf1a W224AW242A-GR & chimeric Ptf1a-GR \\
\hline GABAergic & 4 & $\Longrightarrow$ & $\Longrightarrow$ & $\Uparrow$ \\
\hline glutamatergic & & $\hat{1}$ & 个ि & $\hat{\imath}$ \\
\hline
\end{tabular}

Figure 4.1 Distinct activities of Ptf1a, its mutated and chimeric version as well as of Ngn2 in regard to neuronal subtype specification. Shown are the different Ptf1a constructs as well as Ngn2 and their specificity in neuronal subtype specification. A green arrow represents specification of a neuronal subtype, while a yellow arrow represents no effect on the cell type. In contrast, a red arrow indicates inhibition of a specific neuronal subtype. 


\subsection{Regulation of gene induction of $\mathrm{Su}(\mathrm{H})$-dependent and independent Ptf1a target genes}

The context-specific cooperation with co-factors represents a common mechanism for how bHLH transcription factors are able to exert distinct activities (Bertrand et al., 2002; Powell and Jarman, 2008). However, the regulatory mechanism behind distinct transcriptional complex formation and activity often remains elusive.

The heterodimer of Ptf1a is sufficient to activate neurogenesis, while the heterotrimeric complex is essential for GABAergic inhibitory neuron formation. In contrast to the expectations based on the Nanostring experiments using a limited number of known genes, the RNA-sequencing analysis revealed that Ptf1a also induces $\mathrm{Su}(\mathrm{H})$-dependent target genes after $6 \mathrm{~h}$ of protein induction. However, the number of $\mathrm{Su}(\mathrm{H})$-dependent target genes is highly increased after $25 \mathrm{~h}$. Furthermore, analysis of genes induced by Ptf1a in the presence of CHX through RNA-sequencing showed that, indeed, genes activated by Ptf1a after $25 \mathrm{~h}$ are direct target genes of Ptf1a. Consistent with observation in the mouse, the proneural bHLH transcription factor Ngn2 was shown to be a direct target of the heterotrimeric Ptf1a transcription complex. Ngn2 and Ptf1a are coexpressed in the region of the cerebellum that gives rise to the GABAergic inhibitory neurons (Fig. 3.5). In gain of function assays in $X$. laevis animal caps, the GABAergic neuronal subtype-inducing activity of Ptf1a was found to be dominant over the glutamatergic-inducing activity of Ngn2 (Fig. 3.6). This suggests that Ngn2 may act downstream of Ptf1a to participate in GABAergic interneuron formation. This hypothesis is supported by a slight increase in the number of GABAergic dl4 neurons in the chick neural tube after Ngn2 electroporation, as well as by the absence of this neuronal cell type in Ascl1:Ngn2 double knockout mice, but not in the single Ascl1 knockout mice (Helms et al., 2005).

The delayed activation of direct target genes raises the question, what regulates the timing of activation of Ptf1a direct target genes. In a first attempt to answer this question, it could be shown that neither the expression of $S u(H)$ transcripts alters throughout development, nor that the interaction of Ptf1a and $\mathrm{Su}(\mathrm{H})$ is prevented in vivo (Fig. 3.9). As $\mathrm{Su}(\mathrm{H})$ protein levels may be limiting due to post-transcriptional mechanisms, expression levels of $\mathrm{Su}(\mathrm{H})$ in the embryo 
were increased by microinjection of $\mathrm{Su}(H)$ mRNA together with Ptf1a mRNA (Fig. 3.10 and Fig. 3.21). The assays were analysed by the Nanostring nCounter system and revealed that while co-expression of Ptf1a mRNA and $\mathrm{Su}(\mathrm{H}) \mathrm{mRNA}$ did not result in an earlier onset of gene expression of the $\mathrm{Su}(\mathrm{H})$ dependent target genes (Fig. 3.10 and Fig. 3.21), it did influence quantitatively the level of gene induction. This is important as it demonstrates that the injected $\mathrm{Su}(\mathrm{H})$ protein was functional and suggests that $\mathrm{Su}(\mathrm{H})$ protein availability is not the reason why direct target genes of the trimeric complex are delayed in their activation. As elevated $\mathrm{Su}(\mathrm{H})$ expression levels either increased or decreased the gene induction of Ptf1a Su(H)-dependent target genes (Fig. 3.10 and Fig. 3.21), they may have different threshold or kinetic responses to the heterotrimeric complex. Similarly, Ptf1a target genes in the context of pancreas development respond differently to Ptf1a expression (Fukuda et al., 2008; Dong et al., 2008). Low levels of Ptf1a promote endocrine, while high Ptf1a levels are required for exocrine cell differentiation. Therefore, such a dosage-dependent mechanism might generally apply for the regulation of Ptf1a target gene induction.

The early function of Ptf1a during neuronal differentiation and the late function during neuronal subtype specification are reminiscent of Ptf1a activity during pancreas development. Ptf1a first promotes the commitment of endodermal cells into pancreatic precursors of both, endocrine and exocrine cell lineages (Burlison et al., 2008; Kawaguchi et al., 2002) and later, Ptf1a is required for acinar exocrine cell differentiation (Zecchin et al., 2004; Lin et al., 2004; Kawaguchi et al., 2002). Notch-signalling also plays a key role during pancreas development. It is highly active in exocrine pancreatic precursor cells where it prevents their terminal differentiation (Hald et al., 2003; Murtaugh et al., 2003; Esni et al., 2004). In this regard, it could be shown that the Notchpathway and its effectors of the Hes family inhibit Ptf1a activity (Esni et al., 2004; Ghosh and Leach, 2006). In the nervous system, Notch-signalling in the neural progenitor cells is high and also maintains an undifferentiated cell state. Repression of bHLH transcription factors by the Notch-signalling effectors is a common regulatory mechanism in general neurogenesis (Takke et al., 1999; Schneider et al., 2001) and thus it is possible that also Ptf1a function in the nervous system is regulated by this mechanism. Furthermore, binding of $\mathrm{Su}(\mathrm{H})$ 
by Notch-ICD and Ptf1a was shown to be mutually exclusive (Beres et al., 2006) and therefore, a competition between Ptf1a and Notch-ICD for Su(H)binding may also be involved in the regulation of the expression of the distinct Ptf1a target genes (Henke et al., 2009b). When the Notch-signalling activity declines, $\mathrm{Su}(\mathrm{H})$ might be released from Notch-ICD to form the heterotrimeric complex with Ptf1a. To analyse if a competition between Ptf1a and Notch-ICD controls complex formation and thus target genes, a dominant-negative DII1 (Delta $\left.{ }_{\text {stu }}\right)$ was used to block Notch-signalling (data not shown), however, this did not lead to an alteration in the onset of gene expression of $\mathrm{Su}(\mathrm{H})$-dependent Ptf1a target genes. However, more detailed experiments have to be conducted to analyse this possibility.

In the presence of cycloheximide ( $\mathrm{CHX})$, which blocks protein synthesis, activation of the $\mathrm{Su}(\mathrm{H})$-dependent target gene $\mathrm{Ngn} 2$ was observed within $8.5 \mathrm{~h}$, but not in the absence of $\mathrm{CHX}$ (Fig. 3.16). This suggests that there is a mechanism which prevents early activation of $\mathrm{Su}(\mathrm{H})$-dependent target genes that requires protein synthesis.

The formation of the two distinct Ptf1a transcription complexes might be regulated by posttranslational modification as has been shown for other bHLH proteins. For example, the distinct activities of the bHLH transcription factor Ngn2 were shown to be regulated through posttranslational phosphorylation (Hindley et al., 2012; Ma et al., 2008). In this context, it was also shown that the transcriptional activity of Ptf1a and its cooperation with Rbp-I is dependent on histone acetylation mediated by the acetyltransferase cofactor p/CAF (Rodolosse et al., 2009).

\subsection{Identification of the genetic program regulated by Ptf1a during $X$. laevis neurogenesis}

In contrast to the exocrine pancreas, the genetic regulatory network downstream of Ptf1a in the context of the nervous system is poorly understood. In the murine nervous system, only four direct target genes of Ptf1a have been identified so far, including Ptf1a itself, which undergoes positive autoregulation (Meredith et al., 2009), Ngn2 (Henke et al., 2009b), Kirrel2 and Nephrin (Nishida et al., 2010). Therefore, with the aim of identifying Ptf1a direct targets, genes induced in $X$. laevis animal caps by Ptf1a in the presence of $\mathrm{CHX}$ were 
analysed by RNA-sequencing. The number of genes induced compared to uninjected control caps strongly increased when Ptf1a was co-expressed with $\mathrm{Su}(\mathrm{H})$. The identified direct target genes were compared to genes induced by Ptf1a after 6 or $25 \mathrm{~h}$ in the absence of $\mathrm{CHX}$. Among the identified direct target genes (based on their presence in the CHX RNA-seq experiment) activated by Ptf1a after 25 h were Kirrel2 (Nishida et al., 2010) and Ngn2 (Henke et al., 2009b). However, none of the known transcription factors involved in GABAergic interneuron formation were identified, suggesting that their activation by Ptf1a is indirect. Gene ontology analysis of the direct target genes induced by Ptf1a in combination with $\mathrm{Su}(\mathrm{H})$ revealed that Ptf1a directly induces many transcription factors. Several of them are involved in general neurogenesis, including lateral inhibition and cell-cycle regulation, demonstrating that Ptf1a directly controls early steps of neurogenesis. Additionally, the Ptf1a target genes were compared to a previous Affymetrix microarray analysis screen, which identified 57 direct target genes of $X$. laevis Ngn2-GR in animal caps (Seo et al., 2007). Among the 258 direct genes of Ptf1a-GR alone, 19 genes were also shown by Seo et al. to be direct Ngn2 targets, while for Ptf1a-GR together with $\mathrm{Su}(\mathrm{H})-\mathrm{GR} 24$ out of the 638 direct genes were similarly identified (Appendix 6.4). The shared direct targets of Ptf1a-GR and Ngn2-GR included genes involved in neuronal differentiation (MyT1, NeuroD4) and cell migration (Snail1, Twist1).

Gene ontology analysis of the late target genes demonstrated that Ptf1a directly regulates a wide range of biological processes, including cell adhesion, cell motion and cell projection organisation. A similar wide program of biological processes was identified for the mammalian Ascl1 (Castro et al., 2011) and Atoh1 (Klisch et al., 2011), two proneural transcription factors with a distinct neuronal subtype specification in the vertebrate CNS.

Pancreas specific genes were also present in the group of genes induced by Ptf1a in the presence of $\mathrm{CHX}$. Therefore, the direct target genes induced two-fold or more by Ptf1a-GR and Ptf1a/Su(H)-GR in the RNA-sequencing analysis were compared to published studies identifying Ptf1a targets by ChIPsequencing. In the pancreatic cell line 266-6, which has an expression profile similar to pancreatic progenitor cells, Ptf1a was found to bind regulatory 
elements near 936 genes (Thompson et al., 2012) and near 4632 genes in the adult mouse pancreas (Masui et al., 2010). Of these, in our set of direct target genes 38 genes were identified as Ptf1a-GR/Su(H)-GR and 15 genes as Ptf1aGR direct targets (Appendix 6.4) (Thompson et al., 2012). In contrast, of the putative direct targets in the adult mouse pancreas, only seven were identified as Ptf1a-GR/Su(H)-GR and three as Ptf1a-GR direct target genes (Masui et al., 2010) (Appendix 6.4). In general, the number of overlapping genes was quite low. However, 19 direct candidates (Appendix 6.4) induced by Ptf1a-GR/Su(H)GR were also found to be induced in endodermal explants of stage 32 and 36 $X$. laevis embryos (Bilogan and Horb, 2012), suggesting that genes functioning downstream of Ptf1a during pancreatic development are present in our list of candidate genes.

\subsection{Identification of novel Su(H)-dependent Ptf1a direct target genes}

To understand how Ptf1a induces neuronal subtype specification via the trimeric complex, genes were identified, which are present in both RNAsequencing approaches, but are not activated by Ptf1a $1 \mathrm{~W}^{\mathrm{W} 24 \mathrm{~A} / \mathrm{W} 242 \mathrm{~A}}$. This comparison identified $111 \mathrm{Su}(\mathrm{H})$-dependent Ptf1a target genes, among them Ngn2 and Kirre/2. In a first step to verify selective candidates, eight of the new $\mathrm{Su}(\mathrm{H})$-dependent direct target genes were analysed with the Nanostring nCounter RNA profiling system (Fig. 3.20 and Fig. 3.21, Appendix 6.1.3 and 6.1.4). The selected candidates represent different types of transcription factors and none of them, with the exception of barh/2, which is expressed in amacrine cells in the retina (Ding et al., 2009), were specifically described before as genes associated with GABAergic inhibitory neuronal subtype determination. Nanostring analyses demonstrated that all of the genes analysed were dependent on the interaction of Ptf1a and $\mathrm{Su}(\mathrm{H})$ as the induction by Ptf1a ${ }^{\text {W224A/W242A }}$ was reduced at least to half or completely eliminated compared to wild-type Ptf1a. Similar to the known downstream targets of Ptf1a, the majority of the novel $\mathrm{Su}(\mathrm{H})$-dependent genes were strongly increased when Ptf1a was co-expressed with $\mathrm{Su}(\mathrm{H})$. However, one gene was downregulated upon co-injection of $\mathrm{Su}(\mathrm{H})$. This differential gene induction by Ptf1a alone and in combination with $\mathrm{Su}(\mathrm{H})$ is similar to what we already observed for other Ptf1a 
downstream targets such as Gad1 and Ngn2 (Fig. 3.10). This further supports the hypothesis that different $\mathrm{Su}(\mathrm{H})$-dependent targets may have a different threshold response for the heterotrimeric Ptf1a complex, which regulates this activation. Analysis of additional $\mathrm{Su}(\mathrm{H})$-dependent target genes will allow a classification of Ptf1a target genes into distinct groups and therefore may give insight into how Ptf1a promotes multiple developmental processes. 


\section{Conclusion}

In summary, this study demonstrates that Ptf1a can function as proneural bHLH transcription factor in $X$. laevis and that for this activity, Ptf1a does not require the interaction with $\mathrm{Su}(\mathrm{H})$. Furthermore, through two independent RNAsequencing approaches, the genetic network directly downstream of Ptf1a in the context of general neurogenesis as well as neuronal subtype specification could be defined. Analysis of the direct target genes will give new insight into the mechanism how Ptf1a activates general neurogenesis and neuronal subtype specification and may elucidate new mechanisms by which proneural transcription factors are able to confer distinct activities during the development of the nervous system. 


\section{Bibliography}

Afelik, S., Chen, H. and Pieler, T. (2006). Combined ectopic expression of $\mathrm{Pdx} 1$ and Ptf1a/p48 results in the stable conversion of posterior endoderm into endocrine and exocrine pancreatic tissue. Genes Dev 20, 1441-6.

Al-Shammari, M., Al-Husain, M., Al-Kharfy, T. and Alkuraya, F. S. (2011). A novel PTF1A mutation in a patient with severe pancreatic and cerebellar involvement. Clin Genet 80, 196-8.

Alaynick, H. A., Jessell, T. M. and Pfaff, S. L. (2011). SnapShot: spinal cord development. Cell 146, 178-178 e1.

Alvarez, I. S., Araujo, M. and Nieto, M. A. (1998). Neural induction in whole chick embryo cultures by FGF. Dev Biol 199, 42-54.

Alvarez-Medina, R., Cayuso, J., Okubo, T., Takada, S., and Marti, E. (2008). Wnt canonical pathway restricts graded Shh/Gli patterning activity through the regulation of Gli3 expression. Development 135, 237-47.

Amaya, E. (2005). Xenomics. Genome Res 15, 1683-91.

Anders, S. and Huber, H. (2010). Differential expression analysis for sequence count data. Genome Biol 11, R106.

Bachiller, D., Klingensmith, J., Kemp, C., Belo, J.A., Anderson, R.M., May, S.R., McMahon, J.A., McMahon, A.P., Harland, R.M., Rossant, J., et al. (2000). The organizer factors Chordin and Noggin are required for mouse forebrain development. Nature 403, 658-61.

Bachiller, D., Klingensmith, J., Shneyder, N., Tran, U., Anderson, R., Rossant, J. and De Robertis, E. M. (2003). The role of chordin/Bmp signals in mammalian pharyngeal development and DiGeorge syndrome. Development 130, 3567-78.

Barth, K. A., Kishimoto, H., Rohr, K. B., Seydler, C., Schulte-Merker, S. and Wilson, S. H. (1999). Bmp activity establishes a gradient of positional information throughout the entire neural plate. Development 126, 4977-87.

Bellefroid, E. J., Bourguignon, C., Hollemann, T., Ma, Q., Anderson, D. J., Kintner, C. and Pieler, T. (1996). X-MyT1, a Xenopus C2HC-type zinc finger protein with a regulatory function in neuronal differentiation. Cell 87, 1191-202.

Bellefroid, E. J., Kobbe, A., Gruss, P., Pieler, T., Gurdon, J. B. and Papalopulu, N. (1998). Xiro3 encodes a Xenopus homolog of the Drosophila Iroquois genes and functions in neural specification. EMBO J 17, 191-203.

Beres, T. M., Masui, T., Swift, G. H., Shi, L., Henke, R. M. and MacDonald, R. J. (2006). PTF1 is an organ-specific and Notch-independent basic helix-loophelix complex containing the mammalian Suppressor of Hairless (RBP-J) or its paralogue, RBP-L. Mol Cell Biol 26, 117-30.

Bertrand, N., Castro, D. S. and Guillemot, F. (2002). Proneural genes and the specification of neural cell types. Nat Rev Neurosci 3, 517-30.

Bilogan, C. K. and Horb, M. E. (2012). Microarray analysis of Xenopus endoderm expressing Ptf1a. Genesis.

Borchers A., Pieler T. (2010). Programming pluripotent precursor cells derived from Xenopus embryos to generate specific tissues. Genes, 1(3), 413-426.

Bouwmeester, T., Kim, S., Sasai, H., Lu, B. and De Robertis, E. M. (1996). Cerberus is a head-inducing secreted factor expressed in the anterior endoderm of Spemann's organizer. Nature 382, 595-601.

Boy, S., Souopgui, J., Amato, M. A., Wegnez, M., Pieler, T. and Perron, M. (2004). XSEB4R, a novel RNA-binding protein involved in retinal cell 
differentiation downstream of bHLH proneural genes. Development 131, 85162.

Bray, S. J. (2006). Notch signalling: a simple pathway becomes complex. Nat Rev Mol Cell Biol 7, 678-89.

Brewster, R., Lee, J. and Ruiz i Altaba, A. (1998). Gli/Zic factors pattern the neural plate by defining domains of cell differentiation. Nature 393, 579-83.

Briscoe, J., and Ericson, J. (2001). Specification of neuronal fates in the ventral neural tube. Curr Opin Neurobiol 11, 43-49.

Briscoe, J., Pierani, A., Jessell, T. M. and Ericson, J. (2000). A homeodomain protein code specifies progenitor cell identity and neuronal fate in the ventral neural tube. Cell 101, 435-45.

Brunet, J. F. und Ghysen, A. (1999). Deconstructing cell determination: proneural genes and neuronal identity. Bioessays 21, 313-8.

Burlison, J. S., Long, Q., Fujitani, H., Wright, C. V. and Magnuson, M. A. (2008). Pdx-1 and Ptf1a concurrently determine fate specification of pancreatic multipotent progenitor cells. Dev Biol 316, 74-86.

Castro, D. S. and Guillemot, F. (2011). Old and new functions of proneural factors revealed by the genome-wide characterization of their transcriptional targets. Cell Cycle 10, 4026-31.

Castro, D. S., Martynoga, B., Parras, C., Ramesh, V., Pacary, E., Johnston, C., Drechsel, D., Lebel-Potter, M., Garcia, L. G., Hunt, C. et al. (2011). A novel function of the proneural factor Ascl1 in progenitor proliferation identified by genome-wide characterization of its targets. Genes Dev 25, 930-45.

Cau, E., Gradwohl, G., Casarosa, S., Kageyama, R., and Guillemot, F. (2000). Hes genes regulate sequential stages of neurogenesis in the olfactory epithelium. Development 127, 2323-32.

Chan, H. M. and Jan, H. N. (1999). Presenilins, processing of beta-amyloid precursor protein, and notch signaling. Neuron 23, 201-4.

Chen, Y., Pan, F. C., Brandes, N., Afelik, S., Sölter, M., Pieler, T. (2004). Retinoic acid signaling is essential for pancreas development and promotes endocrine at the expense of exocrine cell differentiation in Xenopus. Dev Biol 271, 144-60.

Cheng, L., Arata, A., Mizuguchi, R., Qian, H., Karunaratne, A., Gray, P. A., Arata, S., Shirasawa, S., Bouchard, M., Luo, P. et al. (2004). Tlx3 and Tlx1 are post-mitotic selector genes determining glutamatergic over GABAergic cell fates. Nat Neurosci 7, 510-7.

Cheng, L., Samad, O. A., Xu, H., Mizuguchi, R., Luo, P., Shirasawa, S., Goulding, M. and Ma, Q. (2005). Lbx1 and Tlx3 are opposing switches in determining GABAergic versus glutamatergic transmitter phenotypes. Nat Neurosci 8, 1510-5.

Chien, C. T., Hsiao, C. D., Jan, L. H. and Jan, H. N. (1996). Neuronal type information encoded in the basic-helix-loop-helix domain of proneural genes. Proc Natl Acad Sci U S A 93, 13239-44.

Chitnis, A., Henrique, D., Lewis, J., Ish-Horowicz, D. and Kintner, C. (1995). Primary neurogenesis in Xenopus embryos regulated by a homologue of the Drosophila neurogenic gene Delta. Nature 375, 761-6.

Chitnis, A. and Kintner, C. (1995). Neural induction and neurogenesis in amphibian embryos. Perspect Dev Neurobiol 3, 3-15.

Chitnis, A. and Kintner, C. (1996). Sensitivity of proneural genes to lateral inhibition affects the pattern of primary neurons in Xenopus embryos. Development 122, 2295-301. 
Cockell, M., Stevenson, B. J., Strubin, M., Hagenbuchle, O. and Wellauer, P. K. (1989). Identification of a cell-specific DNA-binding activity that interacts with a transcriptional activator of genes expressed in the acinar pancreas. $\mathrm{Mol}$ Cell Biol 9, 2464-76.

Coffman, C., Harris, H. and Kintner, C. (1990). Xotch, the Xenopus homolog of Drosophila notch. Science 249, 1438-41.

Damianitsch, K. (2008). Doctoral Thesis: Die Funktion des Wnt Antagonisten XsFRP5 während der frühembryonalen Musterbildung des Entoderms in Xenopus laevis. University of Goettingen, Goettingen

Davis, R. L. and Turner, D. L. (2001). Vertebrate hairy and Enhancer of split related proteins: transcriptional repressors regulating cellular differentiation and embryonic patterning. Oncogene 20, 8342-57.

de la Calle-Mustienes, E., Glavic, A., Modolell, J., and Gomez-Skarmeta, J.L. (2002). Xiro homeoproteins coordinate cell cycle exit and primary neuron formation by upregulating neuronal-fate repressors and downregulating the cellcycle inhibitor XGadd45-gamma. Mech Dev 119, 69-80.

Delaune, E., Lemaire, P. and Kodjabachian, L. (2005). Neural induction in Xenopus requires early FGF signalling in addition to BMP inhibition. Development 132, 299-310.

Ding, Q., Chen, H., Xie, X., Libby, R.T., Tian, N., and Gan, L. (2009). BARHL2 differentially regulates the development of retinal amacrine and ganglion neurons. J Neurosci 29, 3992-4003

Dong, P. D., Provost, E., Leach, S. D. and Stainier, D. H. (2008). Graded levels of Ptf1a differentially regulate endocrine and exocrine fates in the developing pancreas. Genes Dev 22, 1445-50.

Dreau, G. L. and Marti, E. (2012). Dorsal-ventral patterning of the neural tube: A tale of three signals. Dev Neurobiol.

Dubois, L., Bally-Cuif, L., Crozatier, M., Moreau, J., Paquereau, L. and Vincent, A. (1998). XCoe2, a transcription factor of the Col/Olf-1/EBF family involved in the specification of primary neurons in Xenopus. Curr Biol 8, 199209.

Dullin, J. P., Locker, M., Robach, M., Henningfeld, K. A., Parain, K., Afelik, S., Pieler, T. and Perron, M. (2007). Ptf1a triggers GABAergic neuronal cell fates in the retina. BMC Dev Biol 7, 110.

Echelard, Y., Epstein, D.J., St-Jacques, B., Shen, L., Mohler, J., McMahon, J.A., and McMahon, A.P. (1993). Sonic hedgehog, a member of a family of putative signaling molecules, is implicated in the regulation of CNS polarity. Cell 75, 1417-30.

Ellis, P., Fagan, B. M., Magness, S. T., Hutton, S., Taranova, O., Hayashi, S., McMahon, A., Rao, M. and Pevny, L. (2004). SOX2, a persistent marker for multipotential neural stem cells derived from embryonic stem cells, the embryo or the adult. Dev Neurosci 26, 148-65.

Ericson, J., Rashbass, P., Schedl, A., Brenner-Morton, S., Kawakami, A., van Heyningen, V., Jessell, T.M., and Briscoe, J. (1997). Pax6 controls progenitor cell identity and neuronal fate in response to graded Shh signaling. Cell 90, 169-80.

Ericson, J., Briscoe, J., Rashbass, P., van Heyningen, V. and Jessell, T. M. (1997). Graded sonic hedgehog signaling and the specification of cell fate in the ventral neural tube. Cold Spring Harb Symp Quant Biol 62, 451-66. 
Ericson, J., Morton, S., Kawakami, A., Roelink, H. and Jessell, T. M. (1996). Two critical periods of Sonic Hedgehog signaling required for the specification of motor neuron identity. Cell 87, 661-73.

Esni, F., Ghosh, B., Biankin, A. V., Lin, J. H., Albert, M. A., Yu, X., MacDonald, R. J., Civin, C. I., Real, F. X., Pack, M. A. et al. (2004a). Notch inhibits Ptf1 function and acinar cell differentiation in developing mouse and zebrafish pancreas. Development 131, 4213-24.

Ferreiro, B., Skoglund, P., Bailey, A., Dorsky, R. and Harris, H. A. (1993). $\mathrm{XASH} 1$, a Xenopus homolog of achaete-scute: a proneural gene in anterior regions of the vertebrate CNS. Mech Dev 40, 25-36.

Fode, C., Ma, Q., Casarosa, S., Ang, S. L., Anderson, D. J. and Guillemot, F. (2000). A role for neural determination genes in specifying the dorsoventral identity of telencephalic neurons. Genes Dev 14, 67-80.

Fog, C. K., Galli, G. G. and Lund, A. H. (2012). PRDM proteins: important players in differentiation and disease. Bioessays 34, 50-60.

Fujitani, H., Fujitani, S., Luo, H., Qiu, F., Burlison, J., Long, Q., Kawaguchi, H., Edlund, H., MacDonald, R. J., Furukawa, T. et al. (2006). Ptf1a determines horizontal and amacrine cell fates during mouse retinal development. Development 133, 4439-50.

Fukuda, A., Kawaguchi, H., Furuyama, K., Kodama, S., Horiguchi, M., Kuhara, T., Kawaguchi, M., Terao, M., Doi, R., Wright, C. V. et al. (2008). Reduction of Ptf1a gene dosage causes pancreatic hypoplasia and diabetes in mice. Diabetes 57, 2421-31.

Furthauer, M., Thisse, C. and Thisse, B. (1997). A role for FGF-8 in the dorsoventral patterning of the zebrafish gastrula. Development 124, 4253-64.

Gammill, L. S. and Sive, H. (1997). Identification of otx2 target genes and restrictions in ectodermal competence during Xenopus cement gland formation. Development 124, 471-81.

Ge, H., He, F., Kim, K. J., Blanchi, B., Coskun, V., Nguyen, L., Wu, X., Zhao, J., Heng, J. I., Martinowich, K. et al. (2006). Coupling of cell migration with neurogenesis by proneural bHLH factors. Proc Natl Acad Sci U S A 103, 131924.

Geiss G.K. , Bumgarner R.E., Birditt B., Dahl T., Dowidar N., Dunaway D.L., Fell H.P., Ferree S., George R.D., Grogan T., James J.J., Maysuria M., Mitton J.D., Oliveri P., Osborn J.L., Peng T., Ratcliffe A.L., Webster P.J., Davidson E.H., Hood L., Dimitrov K. (2008). Direct multiplexed measurement of gene expression with color-coded probe pairs. Nat. Biotechnol. 26(3), 31725.

Ghosh, B. and Leach, S. D. (2006). Interactions between hairy/enhancer of split-related proteins and the pancreatic transcription factor Ptf1-p48 modulate function of the PTF1 transcriptional complex. Biochem J 393, 679-85.

Ghysen, A. and Dambly-Chaudiere, C. (1989). Genesis of the Drosophila peripheral nervous system. Trends Genet 5, 251-5.

Glasgow, S. M., Henke, R. M., Macdonald, R. J., Wright, C. V. and Johnson, J. E. (2005). Ptf1a determines GABAergic over glutamatergic neuronal cell fate in the spinal cord dorsal horn. Development 132, 5461-9.

Godsave, S. F. and Slack, J. M. (1989). Clonal analysis of mesoderm induction in Xenopus laevis. Dev Biol 134, 486-90.

Gomez-Skarmeta, J. L., Glavic, A., de la Calle-Mustienes, E., Modolell, J. and Mayor, R. (1998). Xiro, a Xenopus homolog of the Drosophila Iroquois complex genes, controls development at the neural plate. EMBO J 17, 181-90. 
Graham, V., Khudyakov, J., Ellis, P. and Pevny, L. (2003). SOX2 functions to maintain neural progenitor identity. Neuron 39, 749-65.

Grunz, H. and Tacke, L. (1989). Neural differentiation of Xenopus laevis ectoderm takes place after disaggregation and delayed reaggregation without inducer. Cell Differ Dev 28, 211-7.

Guillemot, F. (1999). Vertebrate bHLH genes and the determination of neuronal fates. Exp Cell Res 253, 357-64.

Guillemot, F. (2007). Spatial and temporal specification of neural fates by transcription factor codes. Development 134, 3771-80.

Hald, J., Hjorth, J. P., German, M. S., Madsen, O. D., Serup, P. and Jensen, J. (2003). Activated Notch1 prevents differentiation of pancreatic acinar cells and attenuate endocrine development. Dev Biol 260, 426-37.

Haldin, C. E., Nijjar, S., Masse, K., Barnett, M. H. and Jones, E. A. (2003) 'Isolation and growth factor inducibility of the Xenopus laevis Lmx1b gene'. Int $J$ Dev Biol 47(4), 253-62.

Hamburger, V. (1969). Hans Spemann and the organizer concept. Experientia 25, 1121-5.

Hardcastle, Z., Chalmers, A.D., and Papalopulu, N. (2000). FGF-8 stimulates neuronal differentiation through FGFR-4a and interferes with mesoderm induction in Xenopus embryos. Curr Biol 10, 1511-14.

Hardcastle, Z. and Papalopulu, N. (2000). Distinct effects of XBF-1 in regulating the cell cycle inhibitor $\mathrm{p} 27(\mathrm{XIC1})$ and imparting a neural fate. Development 127, 1303-14.

Harland, R.M. (1991). In situ hybridization: an improved whole-mount method for Xenopus embryos. Methods Cell Biol 36, 685-95.

Harland, R. M. and Grainger, R. M. (2011). Xenopus research: metamorphosed by genetics and genomics. Trends Genet 27, 507-15.

Hartenstein, V. (1989). Early neurogenesis in Xenopus: the spatio-temporal pattern of proliferation and cell lineages in the embryonic spinal cord. Neuron 3 , 399-411.

Hartenstein, V. (1993). Early pattern of neuronal differentiation in the Xenopus embryonic brainstem and spinal cord. J Comp Neurol 328, 213-31.

Hatakeyama, J., Tomita, K., Inoue, T., and Kageyama, R. (2001). Roles of homeobox and bHLH genes in specification of a retinal cell type. Development 128, 1313-22.

Hedderich, M. (2008). Diploma Thesis: Charakterisierung der proneuralen Aktivität von Ptf1a/p48 in Xenopus (Characterisation of the proneural activity of Ptf1a/p48 in Xenopus). University of Goettingen, Goettingen

Hellsten, U., Harland, R. M., Gilchrist, M. J., Hendrix, D., Jurka, J., Kapitonov, V., Ovcharenko, I., Putnam, N. H., Shu, S., Taher, L. et al. (2010). The genome of the Western clawed frog Xenopus tropicalis. Science 328, 633-6.

Helms, A. H., Battiste, J., Henke, R. M., Nakada, H., Simplicio, N., Guillemot, F. and Johnson, J. E. (2005). Sequential roles for Mash1 and Ngn2 in the generation of dorsal spinal cord interneurons. Development 132, 2709-19. Helms, A. H. and Johnson, J. E. (2003). Specification of dorsal spinal cord interneurons. Curr Opin Neurobiol 13, 42-9.

Hemmati-Brivanlou, A. and Melton, D. A. (1994). Inhibition of activin receptor signaling promotes neuralization in Xenopus. Cell 77, 273-81. 
Henke, R. M., Meredith, D. M., Borromeo, M. D., Savage, T. K. and Johnson, J. E. (2009a). Ascl1 and Neurog2 form novel complexes and regulate Delta-like3 (DII3) expression in the neural tube. Dev Biol 328, 529-40.

Henke, R. M., Savage, T. K., Meredith, D. M., Glasgow, S. M., Hori, K., Dumas, J., MacDonald, R. J. and Johnson, J. E. (2009b). Neurog2 is a direct downstream target of the Ptf1a-Rbpj transcription complex in dorsal spinal cord. Development 136, 2945-54.

Hindley, C., Ali, F., McDowell, G., Cheng, K., Jones, A., Guillemot, F. and Philpott, A. (2012). Post-translational modification of Ngn2 differentially affects transcription of distinct targets to regulate the balance between progenitor maintenance and differentiation. Development 139, 1718-23.

Hollemann, T. and Pieler, T. (1999). Xpitx-1: a homeobox gene expressed during pituitary and cement gland formation of Xenopus embryos. Mech Dev 88, 249-52.

Hollyday, M., McMahon, J. A. and McMahon, A. P. (1995). Wnt expression patterns in chick embryo nervous system. Mech Dev 52, 9-25.

Hongo, I., Kengaku, M. and Okamoto, H. (1999). FGF signaling and the anterior neural induction in Xenopus. Dev Biol 216, 561-81.

Honjo, T. (1996). The shortest path from the surface to the nucleus: RBP-J kappa/Su(H) transcription factor. Genes Cells 1, 1-9.

Hori, K., Cholewa-Waclaw, J., Nakada, H., Glasgow, S. M., Masui, T., Henke, R. M., Wildner, H., Martarelli, B., Beres, T. M., Epstein, J. A. et al. (2008). A nonclassical bHLH Rbpj transcription factor complex is required for specification of GABAergic neurons independent of Notch signaling. Genes Dev 22, 166-78.

Hori, K. and Hoshino, M. (2012). GABAergic neuron specification in the spinal cord, the cerebellum, and the cochlear nucleus. Neural Plast 2012, 921732.

Hoshino, M. (2006). Molecular machinery governing GABAergic neuron specification in the cerebellum. Cerebellum 5, 193-98.

Hoshino, M., Nakamura, S., Mori, K., Kawauchi, T., Terao, M., Nishimura, H. V., Fukuda, A., Fuse, T., Matsuo, N., Sone, M. et al. (2005). Ptf1a, a bHLH transcriptional gene, defines GABAergic neuronal fates in cerebellum. Neuron 47, 201-13.

Hoveyda, N., Shield, J. P., Garrett, C., Chong, H. K., Beardsall, K., BentsiEnchill, E., Mallya, H. and Thompson, M. H. (1999). Neonatal diabetes mellitus and cerebellar hypoplasia/agenesis: report of a new recessive syndrome. J Med Genet 36, 700-4.

Imayoshi, I. and Kageyama, R. (2011). The role of Notch signaling in adult neurogenesis. Mol Neurobiol 44, 7-12.

Ishibashi, M., Ang, S. L., Shiota, K., Nakanishi, S., Kageyama, R. and Guillemot, F. (1995). Targeted disruption of mammalian hairy and Enhancer of split homolog-1 (HES-1) leads to up-regulation of neural helix-loop-helix factors, premature neurogenesis, and severe neural tube defects. Genes Dev 9, 313648.

lype, T., Taylor, D. G., Ziesmann, S. M., Garmey, J. C., Watada, H. and Mirmira, R. G. (2004). The transcriptional repressor Nkx6.1 also functions as a deoxyribonucleic acid context-dependent transcriptional activator during pancreatic beta-cell differentiation: evidence for feedback activation of the nkx6.1 gene by Nkx6.1. Mol Endocrinol 18, 1363-75.

Jan, H. N. and Jan, L. H. (1994). Neuronal cell fate specification in Drosophila. Curr Opin Neurobiol 4, 8-13. 
Jarman, A. P., Grau, H., Jan, L. H. and Jan, H. N. (1993). atonal is a proneural gene that directs chordotonal organ formation in the Drosophila peripheral nervous system. Cell 73, 1307-21.

Jessell, T. M. (2000). Neuronal specification in the spinal cord: inductive signals and transcriptional codes. Nat Rev Genet 1, 20-9.

Kanekar, S., Perron, M., Dorsky, R., Harris, H. A., Jan, L. H., Jan, H. N. and Vetter, M. L. (1997). Xath5 participates in a network of bHLH genes in the developing Xenopus retina. Neuron 19, 981-94.

Kawaguchi, H., Cooper, B., Gannon, M., Ray, M., MacDonald, R. J. and Wright, C. V. (2002). The role of the transcriptional regulator Ptf1a in converting intestinal to pancreatic progenitors. Nat Genet 32, 128-34.

Kengaku, M. and Okamoto, H. (1995). bFGF as a possible morphogen for the anteroposterior axis of the central nervous system in Xenopus. Development 121, 3121-30.

Kim, P., Helms, A. H., Johnson, J. E. and Zimmerman, K. (1997). XATH-1, a vertebrate homolog of Drosophila atonal, induces a neuronal differentiation within ectodermal progenitors. Dev Biol 187, 1-12.

Klisch, T. J., Xi, H., Flora, A., Wang, L., Li, H. and Zoghbi, H. H. (2011). In vivo Atoh1 targetome reveals how a proneural transcription factor regulates cerebellar development. Proc Natl Acad Sci U S A 108, 3288-93.

Krapp, A., Knofler, M., Frutiger, S., Hughes, G. J., Hagenbuchle, O. and Wellauer, P. K. (1996). The p48 DNA-binding subunit of transcription factor PTF1 is a new exocrine pancreas-specific basic helix-loop-helix protein. EMBO J 15, 4317-29.

Krapp, A., Knofler, M., Ledermann, B., Burki, K., Berney, C., Zoerkler, N., Hagenbuchle, O. and Wellauer, P. K. (1998). The bHLH protein PTF1-p48 is essential for the formation of the exocrine and the correct spatial organization of the endocrine pancreas. Genes Dev 12, 3752-63.

Kroll, K. L. (2007). Geminin in embryonic development: coordinating transcription and the cell cycle during differentiation. Front Biosci 12, 1395-409.

Kroll, K. L., Salic, A. N., Evans, L. M. and Kirschner, M. H. (1998). Geminin, a neuralizing molecule that demarcates the future neural plate at the onset of gastrulation. Development 125, 3247-58.

Kulkarni M.M. (2011). Digital multiplexed gene expression analysis using the NanoString nCounter system. Curr Protoc Mol Biol. 25,Unit25B.10.

Laemmli, U. K. (1970). Cleavage of structural proteins during the assembly of the head of bacteriophage T4. Nature 227(5259), 680-85.

Lamb, T. M. and Harland, R. M. (1995). Fibroblast growth factor is a direct neural inducer, which combined with noggin generates anterior-posterior neural pattern. Development 121, 3627-36.

Lamb, T.M., Knecht, A.K., Smith, W.C., Stachel, S.E., Economides, A.N., Stahl, N., Yancopolous, G.D., and Harland, R.M. (1993). Neural induction by the secreted polypeptide noggin. Science 262, 713-18.

Langmead, B. and Salzberg, S. L. (2012). Fast gapped-read alignment with Bowtie 2. Nat Methods 9, 357-9.

Launay, C., Fromentoux, V., Shi, D. L. and Boucaut, J. C. (1996). A truncated FGF receptor blocks neural induction by endogenous Xenopus inducers. Development 122, 869-80.

Le Dreau, G., Garcia-Campmany, L., Rabadan, M. A., Ferronha, T., Tozer, S., Briscoe, J. and Marti, E. (2012). Canonical BMP7 activity is required for the 
generation of discrete neuronal populations in the dorsal spinal cord. Development 139, 259-68.

Leclerc, C., Daguzan, C., Nicolas, M. T., Chabret, C., Duprat, A. M. and Moreau, M. (1997). L-type calcium channel activation controls the in vivo transduction of the neuralizing signal in the amphibian embryos. Mech Dev 64, 105-10.

Leclerc, C., Webb, S. E., Daguzan, C., Moreau, M, Miller, A. L. (2000). Imaging patterns of calcium transients during neural induction in Xenopus laevis emrbyos. J Cell Sci 113, 3519-29.

Leclerc, C., Lee, M., Webb, S. E., Moreau, M. and Miller, A. L. (2003). Calcium transients triggered by planar signals induce the expression of ZIC3 gene during neural induction in Xenopus. Dev Biol 261, 381-90.

Leclerc, C., Neant, I. and Moreau, M. (2012). The calcium: an early signal that initiates the formation of the nervous system during embryogenesis. Front Mol Neurosci 5, 3.

Lee, S. K. and Pfaff, S.L. (2003). Synchronization of neurogenesis and motor neuron specification by direct coupling of bHLH and homeodomain transcription factors. Neuron 38, 731-45.

Lee, J. E. (1997). Basic helix-loop-helix genes in neural development. Curr Opin Neurobiol 7, 13-20.

Lee, J. E., Hollenberg, S. M., Snider, L., Turner, D. L., Lipnick, N. and Weintraub, H. (1995). Conversion of Xenopus ectoderm into neurons by NeuroD, a basic helix-loop-helix protein. Science 268, 836-44.

Lelievre, E. C., Lek, M., Boije, H., Houille-Vernes, L., Brajeul, V., Slembrouck, A., Roger, J. E., Sahel, J. A., Matter, J. M., Sennlaub, F. et al. (2011). Ptf1a/Rbpj complex inhibits ganglion cell fate and drives the specification of all horizontal cell subtypes in the chick retina. Dev Biol 358, 296308.

Levin, M. (1998). The roles of activin and follistatin signaling in chick gastrulation. Int J Dev Biol 42, 553-9.

Lewis, J. (1996). Neurogenic genes and vertebrate neurogenesis. Curr Opin Neurobiol 6, 3-10.

Li, M., Sipe, C. H., Hoke, K., August, L. L., Wright, M. A. and Saha, M. S. (2006). The role of early lineage in GABAergic and glutamatergic cell fate determination in Xenopus laevis. J Comp Neurol 495, 645-57.

Liem, K. F., Jr., TremmI, G. and Jessell, T. M. (1997). A role for the roof plate and its resident TGFbeta-related proteins in neuronal patterning in the dorsal spinal cord. Cell 91, 127-38.

Liem, K. F., Jr., Tremml, G., Roelink, H. and Jessell, T. M. (1995). Dorsal differentiation of neural plate cells induced by BMP-mediated signals from epidermal ectoderm. Cell 82, 969-79.

Lin, J. H., Biankin, A. V., Horb, M. E., Ghosh, B., Prasad, N. B., Yee, N. S., Pack, M. A. and Leach, S. D. (2004). Differential requirement for ptf1a in endocrine and exocrine lineages of developing zebrafish pancreas. Dev Biol 274, 491-503.

Lindsell, C. E., Boulter, J., diSibio, G., Gossler, A. and Weinmaster, G. (1996). Expression patterns of Jagged, Delta1, Notch1, Notch2, and Notch3 genes identify ligand-receptor pairs that may function in neural development. Mol Cell Neurosci 8, 14-27. 
Linker, C. and Stern, C. D. (2004). Neural induction requires BMP inhibition only as a late step, and involves signals other than FGF and Wnt antagonists. Development 131, 5671-81.

Litingtung, H. and Chiang, C. (2000). Specification of ventral neuron types is mediated by an antagonistic interaction between Shh and Gli3. Nat Neurosci 3, 979-85.

Lo, L., Tiveron, M.C., and Anderson, D.J. (1998). MASH1 activates expression of the paired homeodomain transcription factor Phox $2 a$, and couples pan-neuronal and subtype-specific components of autonomic neuronal identity. Development 125, 609-20.

Louvi, A. and Artavanis-Tsakonas, S. (2006). Notch signalling in vertebrate neural development. Nat Rev Neurosci 7, 93-102.

Ma, Q., Kintner, C. and Anderson, D. J. (1996). Identification of neurogenin, a vertebrate neuronal determination gene. Cell 87, 43-52.

Ma, H. C., Song, M. R., Park, J. P., Henry Ho, H. H., Hu, L., Kurtev, M. V., Zieg, J., Ma, Q., Pfaff, S. L. and Greenberg, M. E. (2008). Regulation of motor neuron specification by phosphorylation of neurogenin 2. Neuron 58, 65-77.

Mandel, M., and Higa, A. (1970). Calcium-dependent bacteriophage DNA infection. J Mol Biol 53, 159-62.

Marchal, L., Luxardi, G., Thome, V. and Kodjabachian, L. (2009). BMP inhibition initiates neural induction via FGF signaling and Zic genes. Proc Natl Acad Sci U S A 106, 17437-42.

Marti, E., Bumcrot, D. A., Takada, R. and McMahon, A. P. (1995a). Requirement of $19 \mathrm{~K}$ form of Sonic hedgehog for induction of distinct ventral cell types in CNS explants. Nature 375, 322-5.

Marti, E., Takada, R., Bumcrot, D. A., Sasaki, H. and McMahon, A. P. (1995b). Distribution of Sonic hedgehog peptides in the developing chick and mouse embryo. Development 121, 2537-47.

Martin, B. L. and Harland, R. M. (2006) 'A novel role for lbx1 in Xenopus hypaxial myogenesis'. Development 133(2), 195-208.

Masui, T., Long, Q., Beres, T. M., Magnuson, M. A. and MacDonald, R. J. (2007). Early pancreatic development requires the vertebrate Suppressor of Hairless (RBPJ) in the PTF1 bHLH complex. Genes Dev 21, 2629-43.

Masui, T., Swift, G. H., Deering, T., Shen, C., Coats, H. S., Long, Q., Elsasser, H. P., Magnuson, M. A. and MacDonald, R. J. (2010). Replacement of Rbpj with Rbpjl in the PTF1 complex controls the final maturation of pancreatic acinar cells. Gastroenterology 139, 270-80.

Masui, T., Swift, G. H., Hale, M. A., Meredith, D. M., Johnson, J. E. and Macdonald, R. J. (2008). Transcriptional autoregulation controls pancreatic Ptf1a expression during development and adulthood. Mol Cell Biol 28, 5458-68.

Matzuk, M. M., Lu, N., Vogel, H., Sellheyer, K., Roop, D. R. and Bradley, A. (1995). Multiple defects and perinatal death in mice deficient in follistatin. Nature 374, 360-3.

McCormick, D. A. and Contreras, D. (2001). On the cellular and network bases of epileptic seizures. Annu Rev Physiol 63, 815-46.

McMahon, J. A., Takada, S., Zimmerman, L. B., Fan, C. M., Harland, R. M. and McMahon, A. P. (1998). Noggin-mediated antagonism of BMP signaling is required for growth and patterning of the neural tube and somite. Genes Dev 12, 1438-52.

Megason, S. G. and McMahon, A. P. (2002). A mitogen gradient of dorsal midline Wnts organizes growth in the CNS. Development 129, 2087-98. 
Meredith, D. M., Masui, T., Swift, G. H., MacDonald, R. J. and Johnson, J. E. (2009). Multiple transcriptional mechanisms control Ptf1a levels during neural development including autoregulation by the PTF1-J complex. J Neurosci 29, 11139-48.

Miyatsuka, T., Matsuoka, T. A., Shiraiwa, T., Yamamoto, T., Kojima, I. and Kaneto, H. (2007). Ptf1a and RBP-J cooperate in activating Pdx1 gene expression through binding to Area III. Biochem Biophys Res Commun 362, 905-9.

Mizuhara, E., Minaki, H., Nakatani, T., Kumai, M., Inoue, T., Muguruma, K., Sasai, H. and Ono, H. (2010). Purkinje cells originate from cerebellar ventricular zone progenitors positive for Neph3 and E-cadherin. Dev Biol 338, 202-14.

Mizuseki, K., Kishi, M., Matsui, M., Nakanishi, S. and Sasai, H. (1998a). Xenopus Zic-related-1 and Sox-2, two factors induced by chordin, have distinct activities in the initiation of neural induction. Development 125, 579-87.

Mizuseki, K., Kishi, M., Shiota, K., Nakanishi, S. and Sasai, H. (1998b). SoxD: an essential mediator of induction of anterior neural tissues in Xenopus embryos. Neuron 21, 77-85.

Moreau, M., Leclerc, C., Gualandris-Parisot, L. and Duprat, A. M. (1994). Increased internal $\mathrm{Ca} 2+$ mediates neural induction in the amphibian embryo. Proc Natl Acad Sci U S A 91, 12639-43.

Mullis, K., Faloona, F., Scharf, S., Saiki, R., Horn, G., and Erlich, H. (1986). Specific enzymatic amplification of DNA in vitro: the polymerase chain reaction. Cold Spring Harb Symp Quant Biol 51 Pt 1, 263-73.

Munoz-Sanjuan, I. and Brivanlou, A.H. (2002). Neural induction, the default model and embryonic stem cells. Nat Rev Neurosci 3, 271-80.

Muroyama, H., Fujihara, M., Ikeya, M., Kondoh, H. and Takada, S. (2002). Wnt signaling plays an essential role in neuronal specification of the dorsal spinal cord. Genes Dev 16, 548-53.

Murre, C., McCaw, P. S. \& Baltimore, D. (1989). A new DNA binding and dimerization motif in immunoglobulin enhancer binding, daughterless, MyoD, and myc proteins. Cell 56, 777-83.

Murre, C., McCaw, P. S., Vaessin, H., Caudy, M., Jan, L. H., Jan, H. N., Cabrera, C. V., Buskin, J. N., Hauschka, S. D., Lassar, A. B. et al. (1989). Interactions between heterologous helix-loop-helix proteins generate complexes that bind specifically to a common DNA sequence. Cell 58, 537-44.

Murtaugh, L. C., Stanger, B. Z., Kwan, K. M. and Melton, D. A. (2003). Notch signaling controls multiple steps of pancreatic differentiation. Proc Natl Acad Sci U S A 100, 14920-5.

Nakata, K., Nagai, T., Aruga, J. and Mikoshiba, K. (1997). Xenopus Zic3, a primary regulator both in neural and neural crest development. Proc Natl Acad Sci U S A 94, 11980-5.

Nakhai, H., Sel, S., Favor, J., Mendoza-Torres, L., Paulsen, F., Duncker, G. I. and Schmid, R. M. (2007). Ptf1a is essential for the differentiation of GABAergic and glycinergic amacrine cells and horizontal cells in the mouse retina. Development 134, 1151-60.

Nguyen, V. H., Trout, J., Connors, S. A., Andermann, P., Weinberg, E. and Mullins, M. C. (2000). Dorsal and intermediate neuronal cell types of the spinal cord are established by a BMP signaling pathway. Development 127, 1209-20. 
Nieber, F., Pieler, T. and Henningfeld, K. A. (2009). Comparative expression analysis of the neurogenins in Xenopus tropicalis and Xenopus laevis. Dev Dyn 238, 451-8.

Niehrs, C. and Pollet, N. (1999). Synexpression groups in eukaryotes. Nature 402, 483-7.

Nieuwkoop, P.D., Faber, J. (Eds.) (1967). Normal table of Xenopus laevis (Daudin). Second Edition. North Holland Publ. Co. Amsterdam.

Nishida, K., Hoshino, M., Kawaguchi, H. and Murakami, F. (2010). Ptf1a directly controls expression of immunoglobulin superfamily molecules Nephrin and Neph3 in the developing central nervous system. J Biol Chem 285, 373-80.

Ohtsuka, T., Ishibashi, M., Gradwohl, G., Nakanishi, S., Guillemot, F. and Kageyama, R. (1999). Hes1 and Hes5 as notch effectors in mammalian neuronal differentiation. EMBO J 18, 2196-207.

Oschwald, R., Richter, K. and Grunz, H. (1991). Localization of a nervous system-specific class II beta-tubulin gene in Xenopus laevis embryos by wholemount in situ hybridization. Int J Dev Biol 35, 399-405.

Papalopulu, N. and Kintner, C. (1996). A posteriorising factor, retinoic acid, reveals that anteroposterior patterning controls the timing of neuronal differentiation in Xenopus neuroectoderm. Development 122, 3409-18.

Parr, B. A., Shea, M. J., Vassileva, G. and McMahon, A. P. (1993). Mouse Wnt genes exhibit discrete domains of expression in the early embryonic CNS and limb buds. Development 119, 247-61.

Pascual, M., Abasolo, I., Mingorance-Le Meur, A., Martinez, A., Del Rio, J. A., Wright, C. V., Real, F. X. and Soriano, E. (2007). Cerebellar GABAergic progenitors adopt an external granule cell-like phenotype in the absence of Ptf1a transcription factor expression. Proc Natl Acad Sci U S A 104, 5193-8.

Patterson, K. D. and Krieg, P. A. (1999). Hox11-family genes XHox11 and XHox11L2 in xenopus: XHox11L2 expression is restricted to a subset of the primary sensory neurons. Dev Dyn 214, 34-43.

Pera, E. M., Ikeda, A., Eivers, E. and De Robertis, E. M. (2003). Integration of IGF, FGF, and anti-BMP signals via Smad1 phosphorylation in neural induction. Genes Dev 17, 3023-8.

Perron, M., Opdecamp, K., Butler, K., Harris, H. A. and Bellefroid, E. J. (1999). X-ngnr-1 and Xath3 promote ectopic expression of sensory neuron markers in the neurula ectoderm and have distinct inducing properties in the retina. Proc Natl Acad Sci U S A 96, 14996-5001.

Pevny, L. and Placzek, M. (2005). SOX genes and neural progenitor identity. Curr Opin Neurobiol 15, 7-13.

Piccolo, S., Agius, E., Leyns, L., Bhattacharyya, S., Grunz, H., Bouwmeester, T., and De Robertis, E.M. (1999). The head inducer Cerberus is a multifunctional antagonist of Nodal, BMP and Wnt signals. Nature 397, 70710.

Piccolo, S., Sasai, H., Lu, B. and De Robertis, E. M. (1996). Dorsoventral patterning in Xenopus: inhibition of ventral signals by direct binding of chordin to BMP-4. Cell 86, 589-98.

Pillai, A., Mansouri, A., Behringer, R., Westphal, H., and Goulding, M. (2007). Lhx1 and Lhx5 maintain the inhibitory-neurotransmitter status of interneurons in the dorsal spinal cord. Development 134, 357-66.

Pitulescu, M., Kessel, M. and Luo, L. (2005). The regulation of embryonic patterning and DNA replication by geminin. Cell Mol Life Sci 62, 1425-33. 
Placzek, M., Yamada, T., Tessier-Lavigne, M., Jessell, T. and Dodd, J. (1991). Control of dorsoventral pattern in vertebrate neural development: induction and polarizing properties of the floor plate. Development Suppl 2, 105-22.

Powell, L. M. and Jarman, A. P. (2008). Context dependence of proneural bHLH proteins. Curr Opin Genet Dev 18, 411-7.

Pozzoli, O., Bosetti, A., Croci, L., Consalez, G. G. and Vetter, M. L. (2001). Xebf3 is a regulator of neuronal differentiation during primary neurogenesis in Xenopus. Dev Biol 233, 495-512.

Roberts, A., Li, H. C. and Soffe, S. R. (2012). A functional scaffold of CNS neurons for the vertebrates: The developing Xenopus laevis spinal cord. Dev Neurobiol 72, 575-84.

Roberts, A. (2000). Early functional organization of spinal neurons in developing lower vertebrates. Brain Res Bull 53, 585-93.

Rodolosse, A., Campos, M. L., Rooman, I., Lichtenstein, M. and Real, F. X. (2009). p/CAF modulates the activity of the transcription factor p48/Ptf1a involved in pancreatic acinar differentiation. Biochem J 418, 463-73.

Roelink, H., Porter, J. A., Chiang, C., Tanabe, H., Chang, D. T., Beachy, P. A. and Jessell, T. M. (1995). Floor plate and motor neuron induction by different concentrations of the amino-terminal cleavage product of sonic hedgehog autoproteolysis. Cell 81, 445-55.

Rogers, C. D., Harafuji, N., Archer, T., Cunningham, D. D. and Casey, E. S. (2009a). Xenopus Sox3 activates sox2 and geminin and indirectly represses Xvent2 expression to induce neural progenitor formation at the expense of nonneural ectodermal derivatives. Mech Dev 126, 42-55.

Rogers, C. D., Moody, S. A. and Casey, E. S. (2009b). Neural induction and factors that stabilize a neural fate. Birth Defects Res C Embryo Today 87, 24962.

Rossi, C. C., Hernandez-Lagunas, L., Zhang, C., Choi, I. F., Kwok, L., Klymkowsky, M. and Artinger, K. B. (2008). Rohon-Beard sensory neurons are induced by BMP4 expressing non-neural ectoderm in Xenopus laevis. Dev Biol 314, 351-61.

Rossignol, E. (2011). Genetics and function of neocortical GABAergic interneurons in neurodevelopmental disorders. Neural Plast 2011, 649325.

Roux, E., Strubin, M., Hagenbuchle, O., and Wellauer, P.K. (1989). The cellspecific transcription factor PTF1 contains two different subunits that interact with the DNA. Genes Dev 3, 1613-24.

Saba, R., Johnson, J.E., and Saito, T. (2005). Commissural neuron identity is specified by a homeodomain protein, Mbh1, that is directly downstream of Math1. Development 132, 2147-55.

Sadowski, I., Ma, J., Triezenberg, S. and Ptashne, M. (1988). GAL4-VP16 is an unusually potent transcriptional activator. Nature 335, 563-4.

Sakabe, N. J., Aneas, I., Shen, T., Shokri, L., Park, S. H., Bulyk, M. L., Evans, S. M. and Nobrega, M. A. (2012). Dual transcriptional activator and repressor roles of TBX20 regulate adult cardiac structure and function. Hum Mol Genet 21, 2194-204.

Sambrook, J. and Russel, D.W. (Eds.) (2001). Molecular Cloning: a laboratory manual. Third Edition. Cold Spring Harbour Laboratory Press, Cold Spring Harbour, New York.

Sanes, D.H., Reh, T.A., Harris, H.A. (Eds.) (2006). Development of the nervous system. Second Edition. Amsterdam. 
Sanger, F., Nicklen, S., and Coulson, A.R. (1977). DNA sequencing with chain-terminating inhibitors. Proc Natl Acad Sci U S A 74, 5463-67.

Sasai, H., Kageyama, R., Tagawa, H., Shigemoto, R. and Nakanishi, S. (1992). Two mammalian helix-loop-helix factors structurally related to Drosophila hairy and Enhancer of split. Genes Dev 6, 2620-34.

Sasai, H., Lu, B., Piccolo, S. and De Robertis, E. M. (1996). Endoderm induction by the organizer-secreted factors chordin and noggin in Xenopus animal caps. EMBO J 15, 4547-55.

Sasai, H., Lu, B., Steinbeisser, H., Geissert, D., Gont, L. K. and De Robertis, E. M. (1994). Xenopus chordin: a novel dorsalizing factor activated by organizer-specific homeobox genes. Cell 79, 779-90.

Sato, S. M. and Sargent, T. D. (1989). Development of neural inducing capacity in dissociated Xenopus embryos. Dev Biol 134, 263-6.

Schlosser, G., Koyano-Nakagawa, N. and Kintner, C. (2002). Thyroid hormone promotes neurogenesis in the Xenopus spinal cord. Dev Dyn 225, 485-98.

Schneider, M. L., Turner, D. L. and Vetter, M. L. (2001). Notch signaling can inhibit Xath5 function in the neural plate and developing retina. Mol Cell Neurosci 18, 458-72.

Schroeter, E. H., Kisslinger, J. A. and Kopan, R. (1998). Notch-1 signalling requires ligand-induced proteolytic release of intracellular domain. Nature 393, 382-6.

Selkoe, D. and Kopan, R. (2003). Notch and Presenilin: regulated intramembrane proteolysis links development and degeneration. Annu Rev Neurosci 26, 565-97.

Sellick, G. S., Barker, K. T., Stolte-Dijkstra, I., Fleischmann, C., Coleman, R. J., Garrett, C., Gloyn, A. L., Edghill, E. L., Hattersley, A. T., Wellauer, P. K. et al. (2004). Mutations in PTF1A cause pancreatic and cerebellar agenesis. Nat Genet 36, 1301-5.

Seo, S. and Kroll, K. L. (2006). Geminin's double life: chromatin connections that regulate transcription at the transition from proliferation to differentiation. Cell Cycle 5, 374-9.

Seo, S., Lim, J. H., Yellajoshyula, D., Chang, L. H. and Kroll, K. L. (2007). Neurogenin and NeuroD direct transcriptional targets and their regulatory enhancers. EMBO J 26, 5093-108.

Sharp, P.A., Sugden, B., and Sambrook, J. (1973). Detection of two restriction endonuclease activities in Haemophilus parainfluenzae using analytical agarose--ethidium bromide electrophoresis. Biochemistry 12, 305563.

Sommer, L., Ma, Q. and Anderson, D. J. (1996). neurogenins, a novel family of atonal-related bHLH transcription factors, are putative mammalian neuronal determination genes that reveal progenitor cell heterogeneity in the developing CNS and PNS. Mol Cell Neurosci 8, 221-41.

Souopgui, J., Solter, M. and Pieler, T. (2002). XPak3 promotes cell cycle withdrawal during primary neurogenesis in Xenopus laevis. EMBO J 21, 642939.

Storey, K. G., Crossley, J. M., De Robertis, E. M., Norris, H. E. and Stern, C. D. (1992). Neural induction and regionalisation in the chick embryo. Development 114, 729-41. 
Storey, K. G., Goriely, A., Sargent, C. M., Brown, J. M., Burns, H. D., Abud, H. M. and Heath, J. K. (1998). Early posterior neural tissue is induced by FGF in the chick embryo. Development 125, 473-84.

Storm, R., Cholewa-Waclaw, J., Reuter, K., Brohl, D., Sieber, M., Treier, M., Muller, T. and Birchmeier, C. (2009). The bHLH transcription factor Olig3 marks the dorsal neuroepithelium of the hindbrain and is essential for the development of brainstem nuclei. Development 136, 295-305.

Streit, A., Berliner, A. J., Papanayotou, C., Sirulnik, A. and Stern, C. D. (2000). Initiation of neural induction by FGF signalling before gastrulation. Nature 406, 74-8.

Streit, A., Lee, K. J., Woo, I., Roberts, C., Jessell, T. M. and Stern, C. D. (1998). Chordin regulates primitive streak development and the stability of induced neural cells, but is not sufficient for neural induction in the chick embryo. Development 125, 507-19.

Sullivan, S.A., Akers, L., and Moody, S.A. (2001). foxD5a, a Xenopus winged helix gene, maintains an immature neural ectoderm via transcriptional repression that is dependent on the C-terminal domain. Dev Biol 232, 439-57.

Taelman, V., Opdecamp, K., Avalosse, B., Ryan, K., Bellefroid, E. J. (2001). Xath2, a bHLH gene expressed during late transition stage of neurogenesis in the forebrain of Xenopus embryos. Mech Dev 101, 199-202.

Taira, M., Otani, H., Saint-Jeannet, J. P. and Dawid, I. B. (1994) 'Role of the LIM class homeodomain protein Xlim-1 in neural and muscle induction by the Spemann organizer in Xenopus'. Nature 372 (6507), 677-9.

Takke, C., Dornseifer, P., v Weizsacker, E. and Campos-Ortega, J. A. (1999). her4, a zebrafish homologue of the Drosophila neurogenic gene $\mathrm{E}(\mathrm{spl})$, is a target of NOTCH signalling. Development 126, 1811-21.

Talikka, M., Perez, S. E. and Zimmerman, K. (2002). Distinct patterns of downstream target activation are specified by the helix-loop-helix domain of proneural basic helix-loop-helix transcription factors. Dev Biol 247, 137-48.

Thompson, N., Gesina, E., Scheinert, P., Bucher, P. and Grapin-Botton, A. (2012). RNA profiling and chromatin immunoprecipitation-sequencing reveal that PTF1a stabilizes pancreas progenitor identity via the control of MNX1/HLXB9 and a network of other transcription factors. Mol Cell Biol 32, 1189-99.

Timmer, J. R., Wang, C. and Niswander, L. (2002). BMP signaling patterns the dorsal and intermediate neural tube via regulation of homeobox and helixloop-helix transcription factors. Development 129, 2459-72.

Towbin, H., Staehelin, T. and Gordon, J. (1979). Electrophoretic transfer of proteins from polyacrylamide gels to nitrocellulose sheets: procedure and some applications. Proc Natl Acad Sci U S A 76, 4350-4.

Turner, D. L. and Weintraub, H. (1994). Expression of achaete-scute homolog 3 in Xenopus embryos converts ectodermal cells to a neural fate. Genes Dev 8, 1434-47.

Tutak, E., Satar, M., Yapicioglu, H., Altintas, A., Narli, N., Herguner, O. and Bayram, H. (2009). A Turkish newborn infant with cerebellar agenesis/neonatal diabetes mellitus and PTF1A mutation. Genet Couns 20, 147-52.

Vallstedt, A., Muhr, J., Pattyn, A., Pierani, A., Mendelsohn, M., Sander, M., Jessell, T. M. and Ericson, J. (2001). Different levels of repressor activity assign redundant and specific roles to $\mathrm{Nkx6}$ genes in motor neuron and interneuron specification. Neuron 31, 743-55. 
Vernon, A. E., Devine, C. and Philpott, A. (2003). The cdk inhibitor p27Xic1 is required for differentiation of primary neurones in Xenopus. Development 130 , 85-92.

Wallingford, J. B. and Harland, R. M. (2001). Xenopus Dishevelled signaling regulates both neural and mesodermal convergent extension: parallel forces elongating the body axis. Development 128, 2581-92.

Wang, S. and Barres, B. A. (2000). Up a notch: instructing gliogenesis. Neuron 27, 197-200.

Wawersik, S., Evola, C. and Whitman, M. (2005). Conditional BMP inhibition in Xenopus reveals stage-specific roles for BMPs in neural and neural crest induction. Dev Biol 277, 425-42.

Wettstein, D. A., Turner, D. L. and Kintner, C. (1997). The Xenopus homolog of Drosophila Suppressor of Hairless mediates Notch signaling during primary neurogenesis. Development 124, 693-702.

Wiebe, P. O., Kormish, J. D., Roper, V. T., Fujitani, H., Alston, N. I., Zaret, K. S., Wright, C. V., Stein, R. H. and Gannon, M. (2007). Ptf1a binds to and activates area III, a highly conserved region of the Pdx1 promoter that mediates early pancreas-wide Pdx1 expression. Mol Cell Biol 27, 4093-104.

Wilson, P. A. and Hemmati-Brivanlou, A. (1995). Induction of epidermis and inhibition of neural fate by Bmp-4. Nature 376, 331-3.

Wilson, S. I. and Edlund, T. (2001). Neural induction: toward a unifying mechanism. Nat Neurosci 4 Suppl, 1161-8.

Wilson, S. I., Graziano, E., Harland, R., Jessell, T. M. and Edlund, T. (2000). An early requirement for FGF signalling in the acquisition of neural cell fate in the chick embryo. Curr Biol 10, 421-9.

Wullimann, M. F., Rink, E., Vernier, P. and Schlosser, G. (2005). Secondary neurogenesis in the brain of the African clawed frog, Xenopus laevis, as revealed by PCNA, Delta-1, Neurogenin-related-1, and NeuroD expression. $J$ Comp Neurol 489, 387-402.

Yamada, M., Terao, M., Terashima, T., Fujiyama, T., Kawaguchi, H., Nabeshima, H. and Hoshino, M. (2007). Origin of climbing fiber neurons and their developmental dependence on Ptf1a. J Neurosci 27, 10924-34.

Yamada, T., Placzek, M., Tanaka, H., Dodd, J. and Jessell, T. M. (1991). Control of cell pattern in the developing nervous system: polarizing activity of the floor plate and notochord. Cell 64, 635-47.

Yu, H., McDonnell, K., Taketo, M. M. and Bai, C. B. (2008). Wnt signaling determines ventral spinal cord cell fates in a time-dependent manner. Development 135, 3687-96.

Yu, Z., Syu, L. J. and Mellerick, D. M. (2005). Contextual interactions determine whether the Drosophila homeodomain protein, Vnd, acts as a repressor or activator. Nucleic Acids Res 33, 1-12.

Zecchin, E., Mavropoulos, A., Devos, N., Filippi, A., Tiso, N., Meyer, D., Peers, B., Bortolussi, M. and Argenton, F. (2004). Evolutionary conserved role of ptf1a in the specification of exocrine pancreatic fates. Dev Biol 268, 17484.

Zimmerman, L. B., De Jesus-Escobar, J. M. and Harland, R. M. (1996). The Spemann organizer signal noggin binds and inactivates bone morphogenetic protein 4. Cell 86, 599-606.

Zordan, P., Croci, L., Hawkes, R. \& Consalez, G. G. (2008). Comparative analysis of proneural gene expression in the embryonic cerebellum. Dev Dyn 237, 1726-35. 


\section{Appendix}

6.1 Summary of processed Nanostring data. Given is the averaged fold change over CC of two independent experiments for each sample and gene, respectively. In a second table, the calculated standard error of the mean (SEM) is given for each sample and gene, respectively. Data were processed as described in Methods and Materials.

\subsubsection{Experiment 3.2}

Table 6.1 Summary of the averaged fold change over CC of two independent Nanostring experiments for each sample and gene

\begin{tabular}{|c|c|c|c|c|c|c|c|c|}
\hline \multirow[b]{2}{*}{ Genes } & \multicolumn{2}{|c|}{$3 \mathrm{~h}$} & \multicolumn{2}{|c|}{$6 \mathrm{~h}$} & \multicolumn{2}{|c|}{$25 \mathrm{~h}$} & \multicolumn{2}{|c|}{$25 \mathrm{~h}$} \\
\hline & Ptf1a & Ngn2 & Ptf1a & Ngn2 & Ptf1a & Ngn2 & Ptf1a -Dex & Ngn2 -Dex \\
\hline Ascl1/Xash1 new & 0,7 & 1,1 & 0,8 & 0,8 & 0,6 & 0,6 & 1,0 & 7,6 \\
\hline Cdknx (p27Xic1) & 5,8 & 2,5 & 4,7 & 2,6 & 1,3 & 1,8 & 1,0 & 1,0 \\
\hline DII1 (Delta1) & 35,0 & 16,0 & 16,7 & 20,2 & 10,4 & 14,0 & 1,1 & 1,7 \\
\hline Ebf2-a/b & 59,4 & 10,1 & 162,4 & 73,8 & 2857,7 & 2361,0 & 1,0 & 5,2 \\
\hline Ebf3 (Coe3) & 1,0 & 1,0 & 1,0 & 1,0 & 85,5 & 184,2 & 1,1 & 5,4 \\
\hline Gad1 & 1,0 & 1,0 & 1,0 & 1,0 & 1069,2 & 10,2 & 1,0 & 1,0 \\
\hline H4 new & 1,1 & 1,3 & 1,2 & 1,2 & 0,7 & 0,7 & 1,1 & 0,9 \\
\hline $\mathrm{Hb} 9 / \mathrm{mn} \times 1$ & 0,4 & 0,3 & 2,5 & 1,9 & 1,0 & 1,0 & 1,0 & 1,0 \\
\hline Hox11L2/TIx3 & 1,5 & 1,0 & 0,7 & 0,5 & 1,0 & 1182,6 & 3,5 & 4,9 \\
\hline Hpx6 (Lbx1) & 1,0 & 1,0 & 1,0 & 1,0 & 11,2 & 1,0 & 1,0 & 1,0 \\
\hline Islet1 (isl1) & 0,8 & 3,0 & 0,4 & 0,7 & 0,2 & 1,9 & 1,1 & 1,1 \\
\hline Lhx1 (Lim1) & 0,2 & 0,1 & 0,4 & 0,4 & 62,3 & 1,0 & 1,0 & 1,0 \\
\hline Lhx2 & 1,0 & 1,0 & 4,9 & 1,0 & 2,8 & 1,0 & 1,0 & 1,0 \\
\hline Lhx5 & 1,1 & 0,8 & 0,9 & 0,8 & 372,2 & 0,7 & 1,0 & 1,0 \\
\hline Lmx1b & 0,4 & 0,6 & 0,5 & 0,8 & 40,8 & 1,0 & 1,0 & 1,0 \\
\hline Mxi1 & 4,3 & 3,3 & 4,5 & 4,2 & 0,5 & 1,9 & 0,8 & 1,2 \\
\hline MyT1 & 31,4 & 16,1 & 12,3 & 12,0 & 3,5 & 8,2 & 1,1 & 2,0 \\
\hline NCAMa/b & 3,4 & 3,1 & 0,4 & 0,8 & 14,0 & 44,6 & 1,4 & 3,1 \\
\hline NeuroD1a/b & 1,0 & 1,0 & 1,0 & 1,0 & 569,0 & 2342,8 & 1,0 & 1,0 \\
\hline NeuroD4 (Xath3) & 1,0 & 1,0 & 1,0 & 14,5 & 945,9 & 5388,3 & 1,0 & 22,5 \\
\hline Ngn1 & 1,0 & 1,0 & 1,0 & 1,0 & 174,7 & 1313,4 & 1,0 & 1,0 \\
\hline Ngn3 & 0,8 & 0,5 & 0,6 & 0,6 & 1,0 & 8,1 & 1,0 & 1,0 \\
\hline Nrgn-1b (Ngn2-b) & 0,7 & 1,1 & 0,7 & 0,7 & 71,4 & 7,8 & 1,0 & 1,0 \\
\hline Numbl & 0,9 & 1,0 & 0,8 & 0,9 & 0,5 & 0,7 & 1,1 & 0,9 \\
\hline ODC & 1,0 & 1,0 & 1,0 & 1,0 & 1,0 & 1,0 & 1,0 & 1,0 \\
\hline PRDM13 & 67,1 & 3,5 & 41,8 & 1,6 & 1719,9 & 32,0 & 4,7 & 1,0 \\
\hline PRDM14 & 1,9 & 2,4 & 1,1 & 4,5 & 73,2 & 2256,9 & 1,0 & 1,0 \\
\hline Pak3 & 1,2 & 0,9 & 0,9 & 0,8 & 216,4 & 972,7 & 0,3 & 0,8 \\
\hline Pax2-a & 1,0 & 1,0 & 1,0 & 1,0 & 183,3 & 0,5 & 1,0 & 1,0 \\
\hline Ptf1a-a/b 5'UTR & 0,8 & 1,0 & 1,1 & 1,1 & 3,8 & 1,9 & 1,5 & 1,0 \\
\hline RBM38/Seb4R & 1,2 & 1,2 & 1,1 & 1,2 & 0,7 & 1,2 & 0,9 & 1,1 \\
\hline RBPJ 3'UTR & 1,0 & 1,0 & 0,9 & 0,9 & 0,7 & 0,7 & 1,1 & 1,0 \\
\hline Sox3 & 1,1 & 1,0 & 0,9 & 0,9 & 2,0 & 0,7 & 1,1 & 1,0 \\
\hline Xbra-a/b & 1,4 & 1,0 & 3,8 & 1,1 & 0,1 & 0,9 & 1,3 & 1,2 \\
\hline actc1 & 1,4 & 1,0 & 3,5 & 4,2 & 0,3 & 2,2 & 1,5 & 1,0 \\
\hline g6pd & 1,0 & 1,0 & 0,9 & 1,0 & 0,6 & 0,9 & 1,0 & 0,9 \\
\hline tubb2b (N-tubulin) & 0,9 & 1,8 & 1,7 & 3,5 & 73,5 & 133,4 & 1,4 & 1,2 \\
\hline vGlut1 & 0,8 & 1,0 & 1,2 & 1,1 & 39,5 & 1670,3 & 1,0 & 1,0 \\
\hline
\end{tabular}


Table 6.2 Summary of the calculated standard error of the mean (SEM) of the fold change shown in table 6.1 for each sample and gene

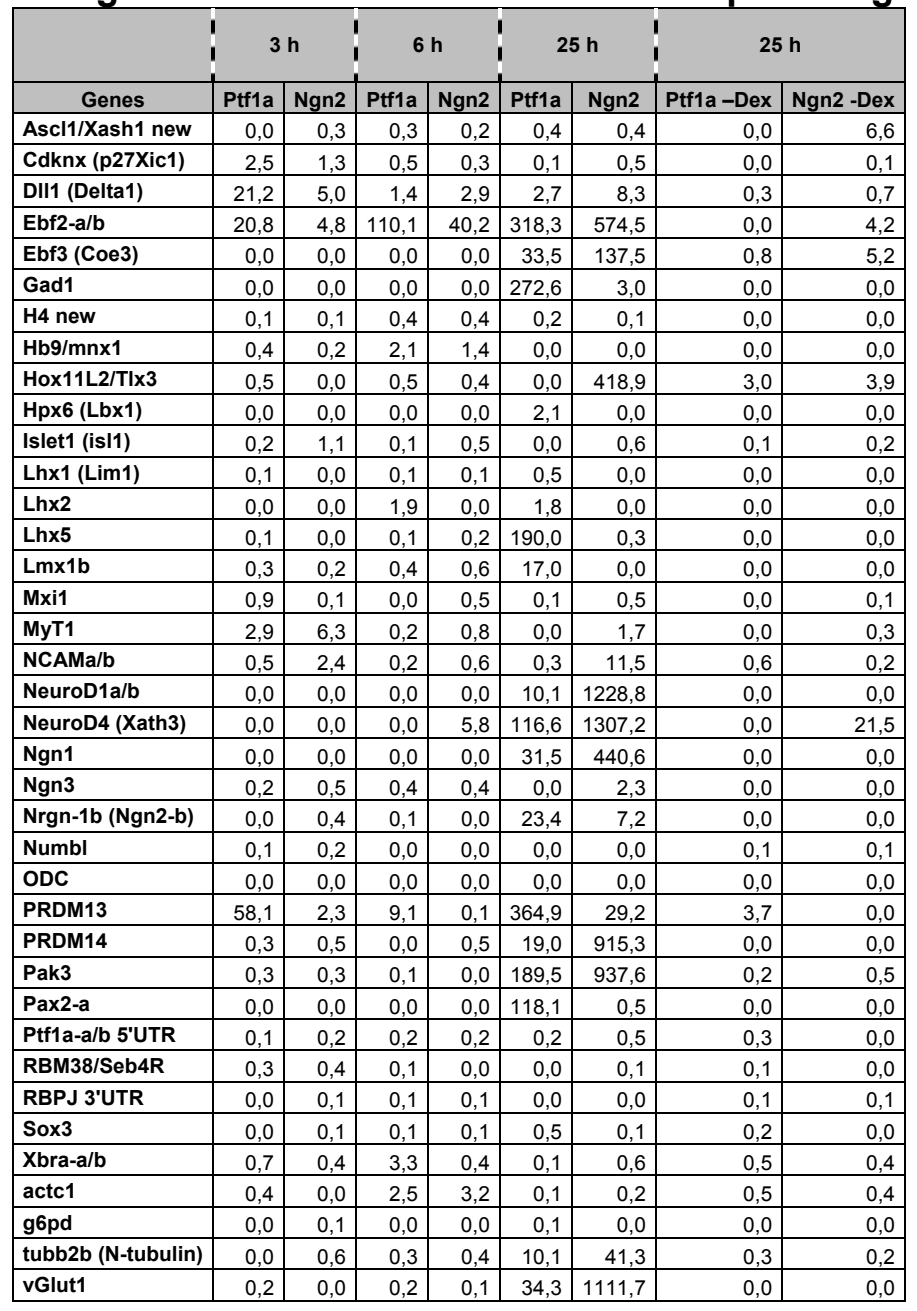




\subsubsection{Experiment 3.7}

Table 6.3 Summary of the averaged fold change over CC of two independent Nanostring experiments for each sample and gene

\begin{tabular}{|c|c|c|c|}
\hline Genes & Ptf1a & Ngn2 & chimeric Ptf1a \\
\hline Arx-a & 0,5 & 0,5 & $\begin{array}{r}0,9 \\
\end{array}$ \\
\hline Ascl1/Xash1 & 0,6 & 0,6 & 0,6 \\
\hline Cdknx (p27Xic1) & 1,8 & 1,6 & 1,8 \\
\hline DII1 (Delta1) & 7,4 & 4,4 & 5,0 \\
\hline Delta2 & 41,4 & 19,9 & 37,3 \\
\hline ESR10 & 91,6 & 88,5 & 50,3 \\
\hline ESR6e (Hes3.1) & 0,9 & 1,6 & 1,2 \\
\hline ESR9.1a & 85,1 & 33,3 & 26,1 \\
\hline Ebf2-a/b & 902,6 & 385,0 & 634,5 \\
\hline Ebf3 (Coe3) & 159,9 & 149,9 & 131,2 \\
\hline Eng1-a/b & 1,0 & 1,0 & 1,0 \\
\hline FGF8 & 12,1 & 3,6 & 4,7 \\
\hline FoxD3a (XfD6) & 2,4 & 1,0 & 2,3 \\
\hline Gad1 & 685,5 & 1,0 & 273,5 \\
\hline H4 & 3,0 & 1,0 & 5,0 \\
\hline $\mathrm{Hb9} / \mathrm{mnx} 1$ & 1,9 & 4,8 & 4,8 \\
\hline Hes1a(Hairy1) & 0,8 & 1,3 & 1,7 \\
\hline Hes2 & 3,5 & 1,0 & 1,0 \\
\hline Hes5.1 (ESR1) & 6,5 & 2,3 & 2,6 \\
\hline Hes6.1 & 1,0 & 0,8 & 1,0 \\
\hline Hes7 (XHR1) & 0,3 & 0,5 & 0,6 \\
\hline Hox11L2/TIx3 & 0,6 & 433,1 & 56,0 \\
\hline Hpx6 (Lbx1) & 20,1 & 2,7 & 4,3 \\
\hline Insm1 & 14,3 & 17,0 & 10,9 \\
\hline Islet1 (is|1) & 0,3 & 1,9 & 1,0 \\
\hline Jagged1 (Serrate-1) & 1,9 & 1,7 & 2,0 \\
\hline Krox20/egr2 & 1,0 & 1,0 & 1,0 \\
\hline Lhx1 (Lim1) & 95,2 & 2,0 & 10,6 \\
\hline Lhx2 & 1,0 & 1,0 & 1,0 \\
\hline Lhx5 & 83,0 & 1,0 & 19,4 \\
\hline Lmx1b & 96,0 & 8,7 & 12,1 \\
\hline Mxi1 & 1,1 & 1,9 & 1,3 \\
\hline МyT1 & 7,4 & 7,5 & 7,9 \\
\hline NCAMa/b & 27,4 & 55,4 & 38,9 \\
\hline NeuroD1a/b & 21,3 & 30,1 & 30,8 \\
\hline NeuroD4 (Xath3) & 606,1 & 1332,2 & 1125,2 \\
\hline NeuroD6 (Xath2) & 1,0 & 1,0 & 1,0 \\
\hline Ngn1 & 178,0 & 317,3 & 289,1 \\
\hline Ngn3 & 0,3 & 2,9 & 0,6 \\
\hline Ngnr-1a (ngn2a) & 1,7 & 1,0 & 1,0 \\
\hline Nkx6.1 & 31,4 & 1,0 & 19,1 \\
\hline Nkx6.2 & 0,7 & 1,0 & 1,3 \\
\hline Notch1 & 1,5 & 1,0 & 1,4 \\
\hline Nrgn-1b (Ngn2-b) & 55,9 & 5,1 & 2,6 \\
\hline Nrp1 & 2,8 & 3,1 & 3,7 \\
\hline Numb & 1,0 & 1,0 & 1,0 \\
\hline Numbl & 0,3 & 0,6 & 0,8 \\
\hline ODC & 1,0 & 1,0 & 1,0 \\
\hline Olig3 & 0,3 & 0,3 & 0,3 \\
\hline Pak3 & 13,2 & 27,3 & 14,8 \\
\hline Pax2-a & 103,4 & 1,0 & 3,9 \\
\hline Pax3 & 139,1 & 18,4 & 33,0 \\
\hline Pax6-a & 2,1 & 0,7 & 1,2 \\
\hline Pax6-b & 23,3 & 0,6 & 0,8 \\
\hline PRDM14 & 2,4 & 54,2 & 9,5 \\
\hline Ptf1a-a/b & 48,8 & 1,0 & 75,7 \\
\hline RBM38/Seb4R & 0,5 & 1,5 & 1,0 \\
\hline RBPJ & 0,6 & 0,8 & 1,0 \\
\hline Slug & 18,9 & 1,0 & 4,5 \\
\hline Sox1 & 0,8 & 0,7 & 1,2 \\
\hline Sox2 & 18,3 & 3,8 & 6,4 \\
\hline Sox3 & 4,2 & 1,1 & 2,0 \\
\hline Sox9-a/b & 26,0 & 1,4 & 4,4 \\
\hline SoxD & 0,7 & 0,7 & 0,9 \\
\hline Twist1-a & 47,8 & 17,3 & 33,7 \\
\hline Xbra-a/b & 0,5 & 2,1 & 1,0 \\
\hline actb & 0,6 & 0,8 & 1,1 \\
\hline actc1 & 2,1 & 3,4 & 4,8 \\
\hline c-myc & 0,3 & 0,7 & 0,7 \\
\hline g6pd & 1,0 & 1,1 & 1,4 \\
\hline gapdh & 0,9 & 2,2 & 1,3 \\
\hline
\end{tabular}




\begin{tabular}{|l|r|r|r|} 
runx1 (Xaml) & 0,2 & 0,6 & 0,5 \\
\hline tubb2b (N-tubulin) & 101,9 & 110,5 & 77,7 \\
\hline vGlut1 & 2,6 & 80,9 & 22,3 \\
\hline
\end{tabular}

Table 6.4 Summary of the calculated standard error of the mean (SEM) of the fold change shown in table 6.3 for each sample and gene

\begin{tabular}{|c|c|c|c|}
\hline Genes & Ptf1a & Ngn2 & chimeric Ptf1a \\
\hline Arx-a & 0,5 & 0,5 & 0,1 \\
\hline Ascl1/Xash1 & 0,4 & 0,4 & 0,4 \\
\hline Cdknx (p27Xic1) & 0,0 & 0,3 & 0,2 \\
\hline DII1 (Delta1) & 3,1 & 1,5 & 0,8 \\
\hline Delta2 & 24,7 & 14,6 & 20,0 \\
\hline ESR10 & 38,2 & 2,8 & 23,7 \\
\hline ESR6e (Hes3.1) & 0,1 & 0,3 & 0,0 \\
\hline ESR9.1a & 54,8 & 13,9 & 10,3 \\
\hline Ebf2-a/b & 56,8 & 81,9 & 44,0 \\
\hline Ebf3 (Coe3) & 22,6 & 70,7 & 40,3 \\
\hline Eng1-a/b & 0,0 & 0,0 & 0,0 \\
\hline FGF8 & 0,3 & 0,6 & 1,4 \\
\hline FoxD3a (XfD6) & 0,2 & 0,2 & 0,5 \\
\hline Gad1 & 101,5 & 0,0 & 9,2 \\
\hline H4 & 2,0 & 0,0 & 4,0 \\
\hline Hb9/mnx1 & 0,7 & 4,5 & 3,1 \\
\hline Hes1a(Hairy1) & 0,1 & 0,2 & 0,0 \\
\hline Hes2 & 2,5 & 0,0 & 0,0 \\
\hline Hes5.1 (ESR1) & 2,7 & 0,0 & 0,4 \\
\hline Hes6.1 & 0,1 & 0,2 & 0,1 \\
\hline Hes7 (XHR1) & 0,2 & 0,0 & 0,3 \\
\hline Hox11L2/TIx3 & 0,4 & 355,0 & 23,3 \\
\hline Hpx6 (Lbx1) & 18,5 & 1,7 & 3,7 \\
\hline Insm1 & 2,1 & 6,5 & 0,1 \\
\hline Islet1 (isl1) & 0,2 & 0,5 & 0,0 \\
\hline Jagged1 (Serrate-1) & 0,3 & 0,2 & 0,1 \\
\hline Krox20/egr2 & 0,0 & 0,0 & 0,0 \\
\hline Lhx1 (Lim1) & 39,6 & 1,0 & 9,6 \\
\hline Lhx2 & 0,0 & 0,0 & 0,0 \\
\hline Lhx5 & 43,8 & 0,0 & 5,3 \\
\hline Lmx1b & 33,0 & 7,7 & 6,4 \\
\hline Mxi1 & 0,1 & 0,2 & 0,3 \\
\hline MyT1 & 1,2 & 1,6 & 0,2 \\
\hline NCAMa/b & 1,2 & 4,7 & 4,4 \\
\hline NeuroD1a/b & 5,7 & 16,1 & 9,2 \\
\hline NeuroD4 (Xath3) & 146,5 & 379,5 & 109,1 \\
\hline NeuroD6 (Xath2) & 0,0 & 0,0 & 0,0 \\
\hline Ngn1 & 47,9 & 72,6 & 72,7 \\
\hline Ngn3 & 0,2 & 1,9 & 0,1 \\
\hline Ngnr-1a (ngn2a) & 0,7 & 0,0 & 0,0 \\
\hline Nkx6.1 & 13,1 & 0,0 & 18,1 \\
\hline Nkx6.2 & 0,1 & 0,0 & 0,2 \\
\hline Notch1 & 0,4 & 0,2 & 0,2 \\
\hline Nrgn-1b (Ngn2-b) & 40,5 & 4,1 & 1,6 \\
\hline Nrp1 & 0,2 & 1,1 & 1,4 \\
\hline Numb & 0,0 & 0,0 & 0,0 \\
\hline Numbl & 0,2 & 0,1 & 0,1 \\
\hline ODC & 0,0 & 0,0 & 0,0 \\
\hline Olig3 & 0,1 & 0,1 & 0,1 \\
\hline Pak3 & 2,7 & 13,5 & 5,2 \\
\hline Pax2-a & 7,6 & 0,0 & 2,9 \\
\hline Pax3 & 44,8 & 17,4 & 24,0 \\
\hline Pax6-a & 1,1 & 0,4 & 0,7 \\
\hline Pax6-b & 17,4 & 0,4 & 0,6 \\
\hline PRDM14 & 1,3 & 16,9 & 1,2 \\
\hline Ptf1a-a/b & 1,3 & 0,0 & 13,4 \\
\hline RBM38/Seb4R & 0,1 & 0,3 & 0,0 \\
\hline RBPJ & 0,1 & 0,1 & 0,0 \\
\hline Slug & 11,7 & 0,0 & 1,2 \\
\hline Sox1 & 0,1 & 0,2 & 0,1 \\
\hline Sox2 & 3,0 & 1,3 & 3,6 \\
\hline Sox3 & 1,9 & 0,3 & 1,3 \\
\hline Sox9-a/b & 13,4 & 0,3 & 2,8 \\
\hline SoxD & 0,1 & 0,1 & 0,1 \\
\hline Twist1-a & 9,3 & 12,4 & 30,5 \\
\hline Xbra-a/b & 0,3 & 1,1 & 0,0 \\
\hline actb & 0,2 & 0,0 & 0,2 \\
\hline actc1 & 1,4 & 0,2 & 1,4 \\
\hline c-myc & 0,1 & 0,2 & 0,0 \\
\hline g6pd & 0,4 & 0,1 & 0,4 \\
\hline
\end{tabular}




\begin{tabular}{|l|r|r|r|} 
gapdh & 0,4 & 0,4 & 0,2 \\
\hline runx1 (Xaml) & 0,0 & 0,0 & 0,0 \\
\hline tubb2b (N-tubulin) & 9,6 & 28,4 & 4,6 \\
\hline vGlut1 & 1,2 & 58,7 & 8,0 \\
\hline
\end{tabular}

\subsubsection{Experiment 3.8 and 3.20}

Table 6.5 Summary of the averaged fold change over CC of two independent Nanostring experiments for each sample and gene

\begin{tabular}{|c|c|c|c|c|}
\hline & & $6 \mathrm{~h}$ & & $32 \mathrm{~h}$ \\
\hline Genes & Ptf1a & Ptf1a $a^{\text {W224AW242A }}$ & Ptf1a & Ptf1a $a^{\text {W224AW242A }}$ \\
\hline Ascl1/Xash1 new & 9,8 & 3,9 & 0,6 & 0,4 \\
\hline Barhl2 & 1,0 & 1,0 & 45,0 & 8,3 \\
\hline Cdknx (p27Xic1) & 7,1 & 5,7 & 2,2 & 1,8 \\
\hline Cpa1 & 1,0 & 1,0 & 2,2 & 0,7 \\
\hline DII1 (Delta1) & 20,0 & 22,2 & 6,0 & 3,0 \\
\hline ESR10 & 9,5 & 0,9 & 6,1 & 4,7 \\
\hline Ebf2-a/b & 234,1 & 51,2 & 2195,2 & 1290,9 \\
\hline Ebf3 (Coe3) & 1,0 & 1,0 & 34,0 & 45,5 \\
\hline FoxD3a (XfD6) & 0,8 & 1,5 & 126,2 & 15,5 \\
\hline Gad1 & 1,0 & 1,0 & 928,7 & 24,4 \\
\hline H4 new & 1,0 & 0,9 & 0,6 & 0,4 \\
\hline HNF6 gene2 & 16,1 & 6,1 & 2261,6 & 1177,2 \\
\hline Hb9/mnx1 & 3,2 & 3,3 & 1,0 & 9,5 \\
\hline Hes5.1 (ESR1) & 34,8 & 6,4 & 214,3 & 136,6 \\
\hline Hmx3 & 3,0 & 1,0 & 19,5 & 0,5 \\
\hline Hox11L2/TIx3 & 5,0 & 12,7 & 17,3 & 219,3 \\
\hline Hpx6 (Lbx1) & 1,0 & 1,0 & 205,8 & 79,2 \\
\hline Islet1 (isl1) & 1,0 & 1,0 & 0,5 & 0,7 \\
\hline Kirrel2 & 3,0 & 0,4 & 51,8 & 1,2 \\
\hline Lhx1 (Lim1) & 10,5 & 9,4 & 171,0 & 36,5 \\
\hline Lhx2 & 0,7 & 0,7 & 34,2 & 23,8 \\
\hline Lhx5 & 0,7 & 0,7 & 274,4 & 2,5 \\
\hline Lmx1b & 4,1 & 0,7 & 66,7 & 51,2 \\
\hline Mecom & 1,0 & 1,0 & 95,5 & 1,9 \\
\hline Mxi1 & 4,7 & 3,4 & 0,7 & 0,6 \\
\hline MyT1 & 27,1 & 14,1 & 4,9 & 4,5 \\
\hline NCAMa/b & 1,5 & 1,2 & 16,5 & 19,5 \\
\hline NeuroD1a/b & 1,0 & 1,0 & 528,4 & 463,3 \\
\hline NeuroD4 (Xath3) & 4,0 & 1,0 & 776,2 & 1210,6 \\
\hline Ngn1 & 1,0 & 1,0 & 217,9 & 315,1 \\
\hline Ngn3 & 7,8 & 4,1 & 1,0 & 1,0 \\
\hline Nrgn-1b (Ngn2-b) & 1,8 & 1,5 & 111,8 & 5,2 \\
\hline ODC & 1,0 & 1,0 & 1,0 & 1,0 \\
\hline PRDM13 & 804,2 & 26,7 & 1768,6 & 71,7 \\
\hline PRDM14 & 1,5 & 2,3 & 135,3 & 761,7 \\
\hline Pak3 & 1,8 & 1,3 & 223,6 & 252,9 \\
\hline Pax2-a & 6,8 & 0,6 & 20,8 & 1,1 \\
\hline Ptf1a-a/b 5'UTR & 13,6 & 6,6 & 4,6 & 0,7 \\
\hline RBM38/Seb4R & 1,6 & 1,8 & 0,8 & 0,6 \\
\hline RBPJ 3'UTR & 1,3 & 1,0 & 0,9 & 0,6 \\
\hline Sox3 & 0,9 & 1,1 & 2,9 & 1,4 \\
\hline Sox9-a/b & 2,3 & 1,3 & 48,8 & 22,6 \\
\hline Pdia2 (XPDip) & 1,0 & 1,0 & 5,3 & 1,0 \\
\hline Xbra-a/b & 2,3 & 2,9 & 0,8 & 0,7 \\
\hline actc1 & 6,9 & 1,1 & 3,2 & 1,9 \\
\hline g6pd & 1,0 & 1,0 & 0,8 & 0,8 \\
\hline tubb2b (N-tubulin) & 3,6 & 1,1 & 161,2 & 259,6 \\
\hline vGlut1 & 1,0 & 1,0 & 23,2 & 523,7 \\
\hline
\end{tabular}


Table 6.6 Summary of the calculated standard error of the mean (SEM) of the fold change shown in table 6.5 for each sample and gene

\begin{tabular}{|c|c|c|c|c|}
\hline \multirow[b]{2}{*}{ Genes } & \multicolumn{2}{|c|}{$6 h$} & \multicolumn{2}{|c|}{$32 \mathrm{~h}$} \\
\hline & Ptf1a & Ptf1a ${ }^{\text {W224AW242A }}$ & Ptf1a & Ptf1a ${ }^{\text {W224A/W242A }}$ \\
\hline Ascl1/Xash1 new & 9,7 & \begin{tabular}{r|}
3,5 \\
\end{tabular} & 0,0 & 0,4 \\
\hline Barhl2 & 0,0 & 0,0 & 42,4 & 8,2 \\
\hline Cdknx (p27Xic1) & 1,5 & 0,9 & 0,9 & 1,3 \\
\hline Cpa1 & 0,0 & 0,0 & 2,1 & 0,3 \\
\hline DII1 (Delta1) & 2,9 & 1,8 & 1,3 & 1,0 \\
\hline ESR10 & 0,3 & 0,2 & 2,4 & 2,8 \\
\hline Ebf2-a/b & 94,1 & 26,0 & 457,0 & 673,6 \\
\hline Ebf3 (Coe3) & 0,0 & 0,0 & 3,2 & 1,7 \\
\hline FoxD3a (XfD6) & 0,3 & 0,5 & 13,9 & 13,6 \\
\hline Gad1 & 0,0 & 0,0 & 43,1 & 0,1 \\
\hline H4 new & 0,0 & 0,1 & 0,0 & 0,2 \\
\hline HNF6 gene2 & 13,0 & 5,5 & 2074,7 & 1133,0 \\
\hline Hb9/mnx1 & 3,0 & 1,2 & 0,0 & 9,4 \\
\hline Hes5.1 (ESR1) & 22,5 & 1,4 & 28,9 & 70,1 \\
\hline $\mathrm{Hm} \times 3$ & 2,0 & 0,0 & 17,9 & 0,5 \\
\hline Hox11L2/TIx3 & 3,2 & 8,4 & 15,8 & 102,4 \\
\hline Hpx6 (Lbx1) & 0,0 & 0,0 & 7,4 & 72,5 \\
\hline Islet1 (isl1) & 0,0 & 0,0 & 0,2 & 0,1 \\
\hline Kirrel2 & 0,6 & 0,1 & 40,0 & 1,1 \\
\hline Lhx1 (Lim1) & 5,7 & 0,9 & 8,3 & 33,4 \\
\hline Lhx2 & 0,3 & 0,3 & 14,5 & 10,2 \\
\hline Lhx5 & 0,2 & 0,2 & 179,7 & 1,5 \\
\hline Lmx1b & 0,7 & 0,3 & 20,6 & 36,0 \\
\hline Mecom & 0,0 & 0,0 & 34,8 & 0,1 \\
\hline Mxi1 & 1,3 & 1,3 & 0,1 & 0,4 \\
\hline MyT1 & 8,4 & 7,5 & 2,9 & 3,0 \\
\hline NCAMa/b & 0,4 & 0,3 & 10,2 & 13,5 \\
\hline NeuroD1a/b & 0,0 & 0,0 & 504,9 & 445,1 \\
\hline NeuroD4 (Xath3) & 3,0 & 0,0 & 263,4 & 556,6 \\
\hline Ngn1 & 0,0 & 0,0 & 180,9 & 283,7 \\
\hline Ngn3 & 6,8 & 3,1 & 0,0 & 0,0 \\
\hline Nrgn-1b (Ngn2-b) & 1,3 & 0,7 & 23,7 & 4,2 \\
\hline ODC & 0,0 & 0,0 & 0,0 & 0,0 \\
\hline PRDM13 & 253,0 & 16,1 & 1194,2 & 67,4 \\
\hline PRDM14 & 0,7 & 1,1 & 117,1 & 726,6 \\
\hline Pak3 & 0,4 & 0,0 & 191,9 & 227,5 \\
\hline Pax2-a & 6,2 & 0,4 & 15,7 & 0,5 \\
\hline Ptf1a-a/b 5'UTR & 12,3 & 5,0 & 3,2 & 0,5 \\
\hline RBM38/Seb4R & 0,0 & 0,1 & 0,2 & 0,2 \\
\hline RBPJ 3'UTR & 0,3 & 0,1 & 0,1 & 0,1 \\
\hline Sox3 & 0,2 & 0,0 & 1,3 & 1,2 \\
\hline Sox9-a/b & 0,6 & 0,2 & 30,4 & 21,1 \\
\hline Pdia2 (XPDip) & 0,0 & 0,0 & 4,3 & 0,0 \\
\hline Xbra-a/b & 1,1 & 2,5 & 0,4 & 0,6 \\
\hline actc1 & 6,1 & 0,1 & 0,4 & 0,8 \\
\hline g6pd & 0,0 & 0,0 & 0,0 & 0,2 \\
\hline tubb2b (N-tubulin) & 1,4 & 0,1 & 106,0 & 172,7 \\
\hline vGlut1 & 0,0 & 0,0 & 0,1 & 61,7 \\
\hline
\end{tabular}




\subsubsection{Experiment 3.10 and 3.21}

Table 6.7 Summary of the averaged fold change over CC of two independent Nanostring experiments for each sample and gene

\begin{tabular}{|c|c|c|c|c|c|c|c|c|c|c|c|c|}
\hline \multirow[b]{2}{*}{ Genes } & \multicolumn{3}{|c|}{ I } & \multicolumn{3}{|c|}{$6 \mathrm{~h}$} & \multicolumn{3}{|c|}{$9 \mathrm{~h}$} & \multicolumn{3}{|c|}{$25 \mathrm{~h}$} \\
\hline & Ptf1a & Ptf1a+Su(H) & $\mathrm{Su}(\mathrm{H})$ & Ptf1a & $\mathrm{Ptf1a}+\mathrm{Su}(\mathrm{H})$ & $\mathrm{Su}(\mathrm{H})$ & Ptf1a & $\mathrm{Ptf1} \mathrm{a}+\mathrm{Su}(\mathrm{H})$ & $\mathrm{Su}(\mathrm{H})$ & Ptf1a & $\mathrm{Ptf1a}+\mathrm{Su}(\mathrm{H})$ & $\mathrm{Su}(\mathrm{H})$ \\
\hline Ascl1/Xash1 new & 1,6 & 2,6 & 1,8 & 0,3 & 0,9 & 0,3 & \begin{tabular}{|l|}
0,6 \\
\end{tabular} & 1,9 & 8,3 & 1,0 & 1,0 & 7,3 \\
\hline Barhl2 & 1,0 & 4,0 & 1,0 & 1,0 & 6,5 & 1,0 & 1,0 & 12,5 & 1,0 & 6,4 & 58,2 & 2,1 \\
\hline Cdknx (p27Xic1) & 6,3 & 5,3 & 1,4 & 3,8 & 3,9 & 1,0 & 3,0 & 3,6 & 1,3 & 1,8 & 1,8 & 1,4 \\
\hline Cpa1 & 1,0 & 1,0 & 1,0 & 1,0 & 1,0 & 1,0 & 1,0 & 1,0 & 1,0 & 0,4 & 2,9 & 1,2 \\
\hline DII1 (Delta1) & 38,7 & 19,2 & 2,8 & 10,9 & 3,8 & 1,4 & 3,9 & 2,8 & 1,1 & 13,9 & 22,1 & 1,2 \\
\hline ESR10 & 28,1 & 58,7 & 1,4 & 16,4 & 26,6 & 2,1 & 40,4 & 58,1 & 3,8 & 446,7 & 1099,4 & 5,0 \\
\hline Ebf2-a/b & 26,4 & 8,4 & 2,5 & 189,1 & 132,2 & 1,0 & 213,3 & 305,4 & 3,0 & 1071,4 & 947,6 & 2,1 \\
\hline Ebf3 (Coe3) & 1,0 & 1,0 & 1,0 & 1,0 & 1,0 & 1,0 & 1,0 & 1,0 & 1,0 & 247,5 & 126,7 & 1,9 \\
\hline FoxD3a (XfD6) & 1,0 & 2,4 & 2,9 & 0,3 & 0,7 & 0,9 & 0,9 & 7,7 & 2,7 & 123,0 & 279,9 & 2,1 \\
\hline Gad1 & 1,0 & 1,0 & 1,0 & 1,0 & 9,7 & 1,0 & 1,0 & 24,4 & 1,0 & 330,0 & 126,3 & 2,1 \\
\hline H4 new & 0,7 & 1,1 & 1,5 & 0,9 & 1,2 & 1,0 & 1,1 & 1,3 & 1,2 & 0,8 & 1,1 & 1,6 \\
\hline HNF6 gene2 & 1,2 & 2,2 & 1,9 & 10,7 & 20,5 & 4,5 & 6,5 & 5,5 & 2,5 & 1090,4 & 1172,9 & 6,7 \\
\hline $\mathrm{Hb} 9 / \mathrm{mnx} 1$ & 4,3 & 8,3 & 3,0 & 1,0 & 17,5 & 1,0 & 1,0 & 6,1 & 1,0 & 1,0 & 1,0 & 2,1 \\
\hline Hes5.1 (ESR1) & 95,7 & 123,8 & 2,2 & 52,7 & 73,7 & 3,8 & 42,6 & 69,2 & 2,2 & 249,9 & 367,9 & 10,4 \\
\hline $\mathrm{Hmx3}$ & 1,0 & 1,3 & 4,4 & 1,0 & 1,0 & 1,0 & 1,0 & 1,0 & 1,3 & 26,7 & 0,8 & 1,8 \\
\hline Hox11L2/TIx3 & 1,0 & 1,0 & 1,0 & 1,0 & 1,0 & 1,0 & 1,0 & 1,0 & 1,0 & 1,0 & 1,0 & 2,1 \\
\hline Hpx6 (Lbx1) & 1,0 & 1,0 & 1,0 & 1,0 & 1,0 & 1,0 & 1,0 & 1,0 & 1,0 & 1,8 & 14,0 & 6,8 \\
\hline Islet1 (isl1) & 1,0 & 1,0 & 1,0 & 1,0 & 1,0 & 1,0 & 1,0 & 1,0 & 1,0 & 0,2 & 0,1 & 1,2 \\
\hline Kirrel2 & 0,8 & 0,8 & 1,1 & 1,3 & 1,5 & 1,3 & 1,2 & 1,6 & 1,0 & 927,1 & 1926,2 & 2,1 \\
\hline Lhx1 (Lim1) & 1,0 & 1,0 & 1,5 & 1,0 & 2,7 & 1,0 & 1,0 & 1,0 & 1,0 & 20,8 & 124,4 & 2,1 \\
\hline Lhx2 & 1,0 & 1,2 & 1,0 & 1,0 & 1,0 & 1,0 & 3,7 & 9,8 & 1,0 & 2,0 & 207,4 & 2,1 \\
\hline Lhx5 & 1,3 & 1,4 & 0,8 & 1,2 & 1,1 & 0,9 & 1,1 & 1,2 & 0,6 & 99,2 & 101,6 & 2,1 \\
\hline Lmx1b & 2,3 & 7,4 & 2,2 & 2,7 & 3,5 & 4,2 & 0,8 & 1,1 & 0,9 & 19,9 & 25,7 & 2,1 \\
\hline Mecom & 1,0 & 1,0 & 1,0 & 1,0 & 1,0 & 1,0 & 1,0 & 3,5 & 1,0 & 82,8 & 1675,8 & 3,9 \\
\hline Mxi1 & 3,5 & 2,5 & 1,5 & 3,4 & 2,4 & 1,4 & 2,8 & 2,1 & 1,4 & 1,0 & 1,5 & 1,4 \\
\hline MyT1 & 12,8 & 12,8 & 1,2 & 13,9 & 16,0 & 0,9 & 11,1 & 19,7 & 0,9 & 3,5 & 5,8 & 0,7 \\
\hline NCAMa/b & 1,3 & 0,8 & 1,4 & 0,9 & 1,1 & 1,4 & 1,8 & 0,9 & 2,0 & 33,5 & 15,6 & 1,8 \\
\hline NeuroD1a/b & 1,0 & 1,0 & 1,0 & 1,0 & 1,0 & 1,0 & 1,0 & 1,1 & 1,0 & 290,3 & 73,1 & 2,1 \\
\hline NeuroD4 (Xath3) & 1,0 & 1,0 & 1,0 & 9,9 & 11,5 & 1,0 & 8,8 & 14,7 & 1,0 & 585,2 & 266,9 & 2,1 \\
\hline Ngn1 & 1,1 & 1,0 & 1,0 & 0,7 & 0,7 & 0,7 & 1,0 & 1,0 & 1,0 & 177,4 & 375,1 & 2,1 \\
\hline Ngn3 & 1,1 & 1,9 & 3,7 & 1,0 & 2,8 & 1,0 & 1,0 & 1,5 & 1,0 & 1,0 & 1,0 & 2,1 \\
\hline Nrgn-1b (Ngn2-b) & 0,6 & 1,2 & 0,6 & 0,5 & 0,9 & 0,4 & 1,8 & 3,9 & 1,0 & 58,5 & 264,4 & 2,1 \\
\hline ODC & 1,0 & 1,0 & 1,0 & 1,0 & 1,0 & 1,0 & 1,0 & 1,0 & 1,0 & 1,0 & 1,0 & 1,0 \\
\hline PRDM13 & 118,0 & 352,3 & 1,0 & 645,2 & 1456,8 & 1,0 & 745,7 & 2056,8 & 1,0 & 1549,8 & 3285,7 & 2,1 \\
\hline PRDM14 & 1,9 & 2,1 & 1,5 & 1,0 & 1,1 & 2,0 & 0,8 & 0,8 & 2,0 & 29,9 & 7,6 & 7,3 \\
\hline Pak3 & 1,1 & 0,7 & 0,8 & 1,0 & 1,4 & 0,8 & 1,6 & 1,9 & 1,0 & 49,9 & 108,0 & 1,0 \\
\hline Pax2-a & 0,7 & 0,7 & 0,7 & 1,0 & 1,3 & 1,0 & 1,0 & 1,0 & 1,0 & 267,5 & 101,2 & 24,4 \\
\hline Ptf1a-a/b 5'UTR & 2,4 & 16,9 & 3,3 & 1,5 & 6,8 & 0,2 & 10,9 & 115,0 & 7,2 & 1,6 & 11,3 & 3,9 \\
\hline RBM38/Seb4R & 1,2 & 1,1 & 0,8 & 1,3 & 1,2 & 0,6 & 2,0 & 1,9 & 1,0 & 0,6 & 1,0 & 0,7 \\
\hline RBPJ 3'UTR & 0,9 & 1,0 & 0,9 & 0,8 & 0,9 & 0,8 & 1,0 & 1,1 & 1,0 & 0,8 & 0,6 & 1,7 \\
\hline Sox3 & 0,9 & 1,0 & 1,0 & 0,8 & 0,9 & 0,8 & 1,0 & 1,0 & 0,9 & 2,3 & 39,7 & 1,6 \\
\hline Sox9-a/b & 1,9 & 3,5 & 1,0 & 1,6 & 5,3 & 1,4 & 1,7 & 5,2 & 1,6 & 41,2 & 81,9 & 1,5 \\
\hline Pdia2 (XPDip) & 1,0 & 1,3 & 1,0 & 1,0 & 20,1 & 1,0 & 6,4 & 88,5 & 1,0 & 2,4 & 743,6 & 2,1 \\
\hline Xbra-a/b & 29,0 & 5,8 & 0,8 & 1,0 & 1,8 & 1,0 & 1,5 & 5,2 & 1,0 & 0,1 & 0,1 & 0,3 \\
\hline actc1 & 0,7 & 6,7 & 9,6 & 1,6 & 1,7 & 0,4 & 6,0 & 1,6 & 1,8 & 0,6 & 0,2 & 1,6 \\
\hline g6pd & 0,9 & 1,0 & 0,9 & 0,9 & 1,0 & 0,9 & 0,9 & 1,0 & 1,0 & 0,8 & 0,5 & 0,9 \\
\hline tubb2b (N-tubulin) & 3,4 & 6,6 & 2,4 & 2,2 & 3,0 & 0,2 & 1,3 & 1,6 & 0,8 & 132,3 & 38,0 & 1,0 \\
\hline vGlut1 & 1,0 & 1,0 & 1,0 & 1,0 & 1,0 & 1,0 & 1,0 & 1,0 & 1,0 & 1,6 & 1,0 & 2,1 \\
\hline
\end{tabular}


Table 6.8 Summary of the calculated standard error of the mean (SEM) of the fold change shown in table 6.7 for each sample and gene

\begin{tabular}{|c|c|c|c|c|c|c|c|c|c|c|c|c|}
\hline \multirow[b]{2}{*}{ Genes } & \multicolumn{3}{|c|}{$3 \mathrm{~h}$} & \multicolumn{3}{|c|}{$6 \mathrm{~h}$} & \multicolumn{3}{|c|}{$9 \mathrm{~h}$} & \multicolumn{3}{|c|}{$25 \mathrm{~h}$} \\
\hline & Ptf1a & \begin{tabular}{|l|}
$\mathrm{Ptf1} 1 \mathrm{a}+\mathrm{Su}(\mathrm{H})$ \\
\end{tabular} & $\mathrm{Su}(\mathrm{H})$ & Ptf1a & Ptf1a+Su(H) & $\mathrm{Su}(\mathrm{H})$ & Ptf1a & Ptf1a+Su(H) & $\mathrm{Su}(\mathrm{H})$ & Ptf1a & Ptf1a+Su(H) & $\mathrm{Su}(\mathrm{H})$ \\
\hline Ascl1/Xash1 new & 1,3 & $\begin{array}{r}0,2 \\
\end{array}$ & 0,4 & 0,3 & \begin{tabular}{r|}
0,2 \\
\end{tabular} & 0,3 & 0,4 & $\begin{array}{r}0,4 \\
\end{array}$ & 6,9 & 0,0 & \begin{tabular}{r|}
0,0 \\
\end{tabular} & 4,1 \\
\hline Barhl2 & 0,0 & 3,0 & 0,0 & 0,0 & 3,5 & 0,0 & 0,0 & 11,5 & 0,0 & 5,4 & 8,1 & 1,1 \\
\hline Cdknx (p27Xic1) & 0,4 & 0,1 & 0,2 & 0,3 & 0,0 & 0,1 & 0,6 & 0,6 & 0,3 & 0,2 & 0,5 & 0,2 \\
\hline Cpa1 & 0,0 & 0,0 & 0,0 & 0,0 & 0,0 & 0,0 & 0,0 & 0,0 & 0,0 & 0,3 & 1,0 & 0,4 \\
\hline DII1 (Delta1) & 1,7 & 6,5 & 0,6 & 3,8 & 1,0 & 0,3 & 0,8 & 0,8 & 0,1 & 0,8 & 1,8 & 0,2 \\
\hline ESR10 & 11,9 & 9,0 & 0,0 & 10,9 & 20,8 & 0,4 & 23,8 & 38,8 & 0,4 & 275,6 & 395,4 & 1,8 \\
\hline Ebf2-a/b & 1,5 & 1,4 & 1,5 & 15,7 & 25,2 & 0,0 & 34,8 & 43,5 & 2,0 & 262,1 & 188,2 & 1,1 \\
\hline Ebf3 (Coe3) & 0,0 & 0,0 & 0,0 & 0,0 & 0,0 & 0,0 & 0,0 & 0,0 & 0,0 & 70,2 & 39,9 & 1,2 \\
\hline FoxD3a (XfD6) & 1,0 & 2,2 & 2,3 & 0,0 & 0,3 & 0,9 & 0,1 & 6,2 & 1,4 & 44,4 & 92,0 & 1,1 \\
\hline Gad1 & 0,0 & 0,0 & 0,0 & 0,0 & 8,7 & 0,0 & 0,0 & 21,5 & 0,0 & 40,3 & 41,5 & 1,1 \\
\hline H4 new & 0,3 & 0,2 & 0,2 & 0,3 & 0,2 & 0,2 & 0,5 & 0,1 & 0,3 & 0,1 & 0,0 & 0,6 \\
\hline HNF6 gene2 & 0,2 & 0,5 & 1,8 & 5,8 & 1,5 & 3,9 & 1,9 & 1,4 & 1,5 & 720,8 & 800,8 & 6,0 \\
\hline $\mathrm{Hb} 9 / \mathrm{mnx} 1$ & 3,3 & 7,3 & 2,0 & 0,0 & 15,0 & 0,0 & 0,0 & 5,1 & 0,0 & 0,0 & 0,0 & 1,1 \\
\hline Hes5.1 (ESR1) & 58,3 & 58,2 & 0,6 & 17,6 & 14,2 & 1,3 & 0,6 & 9,3 & 0,3 & 70,6 & 58,6 & 7,2 \\
\hline $\mathrm{Hmx3}$ & 0,0 & 0,3 & 3,2 & 0,0 & 0,0 & 0,0 & 0,0 & 0,0 & 0,3 & 18,8 & 0,2 & 0,4 \\
\hline Hox11L2/TIx3 & 0,0 & 0,0 & 0,0 & 0,0 & 0,0 & 0,0 & 0,0 & 0,0 & 0,0 & 0,0 & 0,0 & 1,1 \\
\hline Hpx6 (Lbx1) & 0,0 & 0,0 & 0,0 & 0,0 & 0,0 & 0,0 & 0,0 & 0,0 & 0,0 & 0,8 & 9,9 & 5,8 \\
\hline Islet1 (isl1) & 0,0 & 0,0 & 0,0 & 0,0 & 0,0 & 0,0 & 0,0 & 0,0 & 0,0 & 0,0 & 0,0 & 0,2 \\
\hline Kirrel2 & 0,2 & 0,1 & 0,3 & 0,2 & 0,2 & 0,1 & 0,1 & 0,2 & 0,2 & 236,4 & 524,6 & 1,1 \\
\hline Lhx1 (Lim1) & 0,0 & 0,0 & 0,5 & 0,0 & 1,7 & 0,0 & 0,0 & 0,0 & 0,0 & 7,8 & 19,4 & 1,1 \\
\hline Lhx2 & 0,0 & 0,2 & 0,0 & 0,0 & 0,0 & 0,0 & 0,0 & 1,9 & 0,0 & 1,0 & 49,9 & 1,1 \\
\hline Lhx5 & 0,0 & 0,1 & 0,1 & 0,4 & 0,2 & 0,3 & 0,1 & 0,0 & 0,1 & 11,2 & 30,2 & 1,1 \\
\hline Lmx1b & 1,9 & 4,1 & 0,1 & 1,7 & 1,0 & 3,2 & 0,2 & 0,1 & 0,7 & 9,0 & 1,9 & 1,1 \\
\hline Mecom & 0,0 & 0,0 & 0,0 & 0,0 & 0,0 & 0,0 & 0,0 & 2,3 & 0,0 & 58,9 & 1238,8 & 2,9 \\
\hline Mxi1 & 0,9 & 0,1 & 0,1 & 1,2 & 0,6 & 0,4 & 0,3 & 0,2 & 0,2 & 0,1 & 0,2 & 0,3 \\
\hline MyT1 & 4,5 & 6,3 & 0,6 & 0,8 & 0,2 & 0,2 & 0,1 & 0,9 & 0,0 & 0,9 & 0,8 & 0,2 \\
\hline NCAMa/b & 1,1 & 0,2 & 0,6 & 0,8 & 0,6 & 1,1 & 1,6 & 0,7 & 1,7 & 6,4 & 0,7 & 1,0 \\
\hline NeuroD1a/b & 0,0 & 0,0 & 0,0 & 0,0 & 0,0 & 0,0 & 0,0 & 0,1 & 0,0 & 125,7 & 15,0 & 1,1 \\
\hline NeuroD4 (Xath3) & 0,0 & 0,0 & 0,0 & 8,9 & 10,5 & 0,0 & 7,8 & 6,0 & 0,0 & \begin{tabular}{|l|}
251,1 \\
\end{tabular} & 40,6 & 1,1 \\
\hline Ngn1 & 0,1 & 0,0 & 0,0 & 0,3 & 0,3 & 0,3 & 0,0 & 0,0 & 0,0 & 79,0 & 84,1 & 1,1 \\
\hline Ngn3 & 0,1 & 0,9 & 2,7 & 0,0 & 1,2 & 0,0 & 0,0 & 0,5 & 0,0 & 0,0 & 0,0 & 1,1 \\
\hline Nrgn-1b (Ngn2-b) & 0,3 & 0,4 & 0,2 & 0,1 & 0,3 & 0,3 & 0,8 & 1,1 & 0,1 & 19,8 & 38,7 & 1,1 \\
\hline ODC & 0,0 & 0,0 & 0,0 & 0,0 & 0,0 & 0,0 & 0,0 & 0,0 & 0,0 & 0,0 & 0,0 & 0,0 \\
\hline PRDM13 & 34,5 & 66,1 & 0,0 & 199,8 & 204,6 & 0,0 & 290,9 & 600,5 & 0,0 & 417,3 & 822,8 & 1,1 \\
\hline PRDM14 & 0,5 & 0,3 & 0,3 & 0,1 & 0,1 & 0,8 & 0,2 & 0,0 & 0,5 & 7,6 & 6,3 & 4,1 \\
\hline Pak3 & 0,0 & 0,0 & 0,1 & 0,3 & 0,2 & 0,1 & 0,2 & 0,3 & 0,1 & 22,6 & 42,3 & 0,8 \\
\hline Pax2-a & 0,3 & 0,3 & 0,4 & 0,0 & 0,3 & 0,0 & 0,0 & 0,0 & 0,0 & 46,6 & 8,7 & 2,3 \\
\hline Ptf1a-a/b 5'UTR & 2,2 & 14,8 & 2,7 & 1,0 & 4,2 & 0,1 & 8,2 & 93,0 & 6,3 & 0,0 & 1,8 & 3,2 \\
\hline RBM38/Seb4R & 0,5 & 0,3 & 0,2 & 0,2 & 0,2 & 0,1 & 0,3 & 0,2 & 0,1 & 0,1 & 0,1 & 0,1 \\
\hline RBPJ 3'UTR & 0,3 & 0,2 & 0,1 & 0,1 & 0,2 & 0,2 & 0,1 & 0,0 & 0,0 & 0,0 & 0,1 & 0,7 \\
\hline Sox3 & 0,2 & 0,1 & 0,1 & 0,0 & 0,0 & 0,1 & 0,1 & 0,1 & 0,0 & 0,2 & 3,8 & 0,8 \\
\hline Sox9-a/b & 0,5 & 0,3 & 0,1 & 0,3 & 2,0 & 0,3 & 0,1 & 1,2 & 0,1 & 12,7 & 3,3 & 0,8 \\
\hline Pdia2 (XPDip) & 0,0 & 0,3 & 0,0 & 0,0 & 2,6 & 0,0 & 5,4 & 48,2 & 0,0 & 1,4 & 215,4 & 1,1 \\
\hline Xbra-a/b & 28,7 & 5,5 & 0,2 & 0,0 & 0,8 & 0,0 & 0,5 & 4,2 & 0,0 & 0,0 & 0,0 & 0,1 \\
\hline actc1 & 0,3 & 4,6 & 5,7 & 0,3 & 1,3 & 0,2 & 5,0 & 0,6 & 1,6 & 0,2 & 0,0 & 0,2 \\
\hline g6pd & 0,2 & 0,0 & 0,1 & 0,1 & 0,0 & 0,1 & 0,1 & 0,0 & 0,0 & 0,2 & 0,1 & 0,1 \\
\hline tubb2b (N-tubulin) & 0,0 & 1,0 & 0,9 & 1,3 & 1,5 & 0,0 & 0,7 & 0,1 & 0,3 & 79,2 & 16,7 & 0,1 \\
\hline vGlut1 & 0,0 & 0,0 & 0,0 & 0,0 & 0,0 & 0,0 & 0,0 & 0,0 & 0,0 & 0,6 & 0,0 & 1,1 \\
\hline
\end{tabular}


6.2 Summary of the primary Nanostring data. Given are the primary data for each individual Nanostring experiment for each sample and gene.

\subsubsection{Experiment 3.2}

Table 6.9 Primary data of the first Nanostring experiment

\begin{tabular}{|c|c|c|c|c|c|c|c|c|c|c|c|c|}
\hline & & $3 \mathrm{~h}$ & 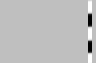 & & $6 \mathrm{~h}$ & I & & $25 \mathrm{~h}$ & & 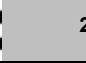 & 25 h -Dex & \\
\hline Genes & Ptf1a & Ngn2 & CC & Ptf1a & Ngn2 & CC & Ptf1a & Ngn2 & CC & Ptf1a & Ngn2 & CC \\
\hline Ascl1/Xash1 new & 21 & 27 & 28 & 28 & 21 & 18 & 8 & 1 & 8 & 6 & 4 & 4 \\
\hline Cdknx (p27Xic1) & 1.125 & 415 & 160 & 1.168 & 799 & 230 & 1.821 & 951 & 544 & 476 & 591 & 589 \\
\hline DII1 (Delta1) & 4.216 & 1.280 & 96 & 2.364 & 3.473 & 132 & 5.894 & 2.177 & 283 & 246 & 343 & 338 \\
\hline Ebf2-a/b & 102 & 16 & 13 & 345 & 221 & 15 & 4.732 & 1.351 & 5 & 2 & 9 & 3 \\
\hline Ebf3 (Coe3) & 2 & 4 & 5 & 6 & 16 & 2 & 2.282 & 1.040 & 21 & 16 & 15 & 26 \\
\hline Gad1 & 1 & 3 & 1 & 2 & 2 & 1 & 2.012 & 15 & 2 & 7 & 4 & 4 \\
\hline H4 new & 234.325 & 238.913 & 268.862 & 258.756 & 281.627 & 264.463 & 99.148 & 60.339 & 69.450 & 64.557 & 67.722 & 71.375 \\
\hline Hb9/mnx1 & 8 & 18 & 32 & 17 & 17 & 13 & 5 & 1 & 1 & 3 & 1 & 1 \\
\hline Hox11L2/TIx3 & 10 & 7 & 10 & 11 & 12 & 15 & 9 & 583 & 3 & 16 & 6 & 2 \\
\hline Hpx6 (Lbx1) & 3 & 2 & 1 & 4 & 3 & 2 & 42 & 1 & 2 & 3 & 1 & 1 \\
\hline Islet1 (is|1) & 12 & 13 & 14 & 15 & 21 & 14 & 298 & 774 & 428 & 508 & 587 & 617 \\
\hline Lhx1 (Lim1) & 15 & 6 & 24 & 11 & 8 & 12 & 114 & 1 & 1 & 1 & 1 & 1 \\
\hline Lhx2 & 8 & 3 & 1 & 25 & 7 & 3 & 29 & 1 & 1 & 1 & 1 & 1 \\
\hline Lhx5 & 1.513 & 1.088 & 1.781 & 1.450 & 1.088 & 1.505 & 856 & 5 & 5 & 3 & 2 & 3 \\
\hline Lmx1b & 10 & 14 & 23 & 12 & 17 & 17 & 108 & 1 & 3 & 1 & 3 & 1 \\
\hline Mxi1 & 1.523 & 781 & 337 & 1.890 & 1.930 & 343 & 829 & 1.414 & 730 & 461 & 819 & 686 \\
\hline MyT1 & 1.679 & 396 & 67 & 2.051 & 2.101 & 143 & 3.285 & 3.001 & 341 & 382 & 776 & 432 \\
\hline NCAMa/b & 17 & 9 & 15 & 18 & 15 & 15 & 2.665 & 3.117 & 73 & 65 & 123 & 41 \\
\hline NeuroD1a/b & 3 & 3 & 3 & 2 & 5 & 2 & 851 & 846 & 2 & 1 & 1 & 1 \\
\hline NeuroD4 (Xath3) & 6 & 1 & 1 & 18 & 39 & 1 & 1.252 & 3.074 & 1 & 2 & 6 & 1 \\
\hline Ngn1 & 1 & 5 & 3 & 1 & 1 & 2 & 328 & 665 & 1 & 1 & 1 & 1 \\
\hline Ngn3 & 10 & 3 & 6 & 2 & 15 & 7 & 3 & 14 & 1 & 1 & 1 & 1 \\
\hline Nrgn-1b (Ngn2-b) & 127 & 200 & 182 & 137 & 164 & 179 & 270 & 10 & 6 & 3 & 3 & 3 \\
\hline Numbl & 781 & 743 & 847 & 847 & 909 & 833 & 706 & 511 & 567 & 740 & 752 & 881 \\
\hline ODC & 23.923 & 19.286 & 26.463 & 25.529 & 24.940 & 20.340 & 31.638 & 16.020 & 11.606 & 14.529 & 17.972 & 17.380 \\
\hline PRDM13 & 674 & 36 & 19 & 1.548 & 67 & 34 & 7.258 & 17 & 7 & 11 & 10 & 8 \\
\hline PRDM14 & 570 & 610 & 291 & 368 & 1.304 & 276 & 159 & 1.017 & 4 & 5 & 2 & 3 \\
\hline Pak3 & 249 & 164 & 189 & 258 & 252 & 237 & 826 & 541 & 16 & 14 & 19 & 14 \\
\hline Pax2-a & 2 & 3 & 8 & 11 & 4 & 7 & 1.081 & 8 & 11 & 3 & 4 & 4 \\
\hline Ptf1a-a/b 5'UTR & 23 & 22 & 26 & 35 & 32 & 26 & 143 & 31 & 16 & 27 & 22 & 19 \\
\hline RBM38/Seb4R & 1.670 & 1.490 & 1.249 & 1.726 & 2.034 & 1.327 & 337 & 267 & 173 & 214 & 329 & 302 \\
\hline RBPJ 3'UTR & 1.957 & 1.789 & 2.242 & 1.978 & 2.049 & 1.907 & 1.416 & 692 & 722 & 846 & 1.060 & 1.054 \\
\hline Sox3 & 10.671 & 9.240 & 11.328 & 11.248 & 9.970 & 10.602 & 766 & 115 & 111 & 96 & 128 & 130 \\
\hline Xbra-a/b & 29 & 25 & 42 & 27 & 28 & 27 & 16 & 53 & 91 & 37 & 47 & 50 \\
\hline actc1 & 14 & 8 & 7 & 15 & 10 & 8 & 84 & 306 & 114 & 247 & 204 & 304 \\
\hline g6pd & 1.633 & 1.401 & 1.764 & 1.652 & 1.702 & 1.457 & 651 & 407 & 330 & 427 & 523 & 508 \\
\hline tubb2b (N-tubulin) & 26 & 40 & 30 & 46 & 97 & 27 & 12.092 & 6.745 & 58 & 190 & 133 & 134 \\
\hline vGlut1 & 9 & 12 & 11 & 8 & 15 & 8 & 30 & 429 & 2 & 8 & 6 & 4 \\
\hline NEG_A & 7 & 2 & 4 & 6 & 3 & 3 & 6 & 4 & 2 & 6 & 8 & 4 \\
\hline NEG_B & 3 & 4 & 4 & 9 & 2 & 9 & 4 & 3 & 3 & 3 & 3 & 2 \\
\hline NEG_C & 3 & 2 & 6 & 5 & 6 & 1 & 4 & 2 & 4 & 3 & 5 & 5 \\
\hline NEG_D & 2 & 4 & 1 & 7 & 7 & 6 & 7 & 9 & 5 & 10 & 6 & 5 \\
\hline NEG_E & 3 & 4 & 6 & 6 & 6 & 5 & 6 & 3 & 3 & 9 & 3 & 3 \\
\hline NEG_F & 9 & 11 & 10 & 11 & 12 & 5 & 16 & 13 & 8 & 17 & 14 & 11 \\
\hline NEG_G & 8 & 10 & 7 & 13 & 10 & 8 & 6 & 3 & 8 & 5 & 1 & 1 \\
\hline NEG_H & 7 & 7 & 5 & 3 & 4 & 6 & 9 & 4 & 2 & 1 & 1 & 1 \\
\hline POS_A & 10.882 & 12.610 & 11.204 & 12.750 & 11.576 & 12.511 & 14.805 & 13.681 & 14.149 & \begin{tabular}{|l|}
15.447 \\
\end{tabular} & 14.521 & 12.036 \\
\hline POS_B & 5.928 & 6.360 & 5.864 & 6.497 & 5.732 & \begin{tabular}{l|l|}
6.389 \\
\end{tabular} & 7.356 & 6.590 & 6.838 & \begin{tabular}{|l|}
7.745 \\
\end{tabular} & \begin{tabular}{|l|}
8.548 \\
\end{tabular} & 6.456 \\
\hline POS_C & 1.653 & 1.790 & 1.611 & 1.807 & 1.630 & 1.721 & 2.058 & 1.870 & 1.894 & 2.181 & 2.356 & 1.780 \\
\hline POS_D & 412 & 402 & 347 & 434 & 366 & 381 & 478 & 429 & 420 & 470 & 494 & 380 \\
\hline POS_E & 68 & 79 & 61 & 95 & 65 & 86 & 78 & 84 & 91 & 89 & 101 & 81 \\
\hline POS_F & 48 & 45 & 36 & 26 & 33 & 30 & 62 & 43 & 52 & 51 & 52 & 46 \\
\hline
\end{tabular}


Table 6.10 Primary data of the second Nanostring experiment

\begin{tabular}{|c|c|c|c|c|c|c|c|c|c|c|c|c|}
\hline I & & $3 \mathrm{~h}$ & I & & $6 \mathrm{~h}$ & I & & $25 \mathrm{~h}$ & I & & 25 h -Dex & \\
\hline Genes & Ptf1a & Ngn2 & CC & Ptf1a & Ngn2 & CC & Ptf1a & Ngn2 & CC & Ptf1a & Ngn2 & CC \\
\hline Ascl1/Xash1 new & 36 & 40 & 62 & 33 & 32 & 24 & 1 & 2 & 5 & 4 & 22 & 4 \\
\hline Cdknx (p27Xic1) & 690 & 243 & 253 & 1.733 & 519 & 184 & 1.188 & 1.767 & 606 & 512 & 562 & 489 \\
\hline DII1 (Delta1) & 1.768 & 1.282 & 162 & 2.740 & 1.701 & 86 & 2.682 & 3.850 & 148 & 206 & 393 & 153 \\
\hline Ebf2-a/b & 41 & 22 & 11 & 372 & 107 & 4 & 3.273 & 3.190 & 4 & 3 & 18 & 6 \\
\hline Ebf3 (Coe3) & 1 & 3 & 2 & 2 & 6 & 5 & 1.418 & 3.211 & 18 & 9 & 19 & 4 \\
\hline Gad1 & 3 & 1 & 1 & 2 & 3 & 3 & 1.038 & 29 & 5 & 6 & 4 & 3 \\
\hline H4 new & 241.378 & 244.122 & 227.724 & 376.957 & 256.086 & 131.144 & 86.171 & 68.804 & 66.216 & 73.555 & 64.327 & 64.809 \\
\hline $\mathrm{Hb} 9 / \mathrm{mnx} 1$ & 34 & 15 & 52 & 49 & 26 & 10 & 6 & 8 & 2 & 1 & 1 & 3 \\
\hline Hox11L2/TIx3 & 11 & 7 & 15 & 18 & 12 & 8 & 6 & 1.747 & 4 & 6 & 24 & 7 \\
\hline Hpx6 (Lbx1) & 2 & 3 & 1 & 1 & 1 & 3 & 28 & 3 & 1 & 1 & 1 & 1 \\
\hline Islet1 (is|1) & 8 & 16 & 17 & 16 & 6 & 9 & 205 & 1.960 & 636 & 519 & 574 & 437 \\
\hline Lhx1 (Lim1) & 16 & 17 & 72 & 28 & 20 & 35 & 97 & 3 & 1 & 1 & 1 & 1 \\
\hline Lhx2 & 9 & 3 & 4 & 17 & 4 & 2 & 4 & 1 & 3 & 1 & 2 & 1 \\
\hline Lhx5 & 1.074 & 682 & 1.017 & 1.398 & 872 & 711 & 546 & 13 & 12 & 5 & 9 & 3 \\
\hline Lmx1b & 25 & 26 & 40 & 29 & 26 & 16 & 47 & 8 & 2 & 1 & 1 & 1 \\
\hline Mxi1 & 841 & 757 & 292 & 2.390 & 1.293 & 289 & 418 & 1.330 & 444 & 379 & 607 & 424 \\
\hline MyT1 & 419 & 304 & 32 & 1.490 & 866 & 69 & 2.214 & 5.273 & 436 & 373 & 847 & 346 \\
\hline NCAMa/b & 18 & 22 & 18 & 24 & 25 & 15 & 2.172 & 7.437 & 116 & 43 & 163 & 54 \\
\hline NeuroD1a/b & 1 & 2 & 1 & 7 & 8 & 3 & 759 & 3.878 & 1 & 1 & 2 & 1 \\
\hline NeuroD4 (Xath3) & 1 & 3 & 1 & 11 & 17 & 1 & 1.379 & 7.257 & 3 & 3 & 47 & 1 \\
\hline Ngn1 & 2 & 3 & 2 & 1 & 1 & 1 & 200 & 1.912 & 1 & 1 & 1 & 1 \\
\hline Ngn3 & 14 & 11 & 26 & 11 & 7 & 10 & 1 & 26 & 1 & 1 & 1 & 1 \\
\hline Nrgn-1b (Ngn2-b) & 146 & 135 & 232 & 144 & 93 & 99 & 78 & 31 & 2 & 1 & 1 & 1 \\
\hline Numbl & 720 & 618 & 977 & 1.019 & 667 & 635 & 750 & 964 & 1.061 & 873 & 816 & 713 \\
\hline ODC & 15.778 & 14.327 & 18.146 & 25.357 & 16.449 & 13.544 & 24.683 & 20.819 & 16.692 & 14.987 & 16.066 & 15.075 \\
\hline PRDM13 & 116 & 24 & 29 & 942 & 38 & 21 & 2.690 & 81 & 7 & 14 & 5 & 4 \\
\hline PRDM14 & 183 & 197 & 140 & 166 & 456 & 80 & 86 & 3.446 & 1 & 3 & 7 & 1 \\
\hline Pak3 & 69 & 56 & 99 & 105 & 62 & 57 & 537 & 2.081 & 5 & 9 & 13 & 14 \\
\hline Pax2-a & 6 & 4 & 7 & 5 & 7 & 6 & 403 & 3 & 4 & 2 & 10 & 2 \\
\hline Ptf1a-a/b 5'UTR & 43 & 45 & 69 & 63 & 40 & 25 & 80 & 50 & 22 & 22 & 23 & 18 \\
\hline RBM38/Seb4R & 1.033 & 970 & 1.439 & 1.579 & 1.028 & 710 & 201 & 297 & 191 & 139 & 176 & 147 \\
\hline RBPJ 3'UTR & 1.490 & 1.301 & 1.779 & 1.833 & 1.217 & 1.018 & 1.111 & 1.054 & 1.138 & 875 & 888 & 763 \\
\hline Sox3 & 9.577 & 7.820 & 10.190 & 14.031 & 9.105 & 7.517 & 235 & 87 & 108 & 105 & 90 & 80 \\
\hline Xbra-a/b & 68 & 47 & 48 & 82 & 19 & 11 & 36 & 139 & 76 & 95 & 91 & 55 \\
\hline actc1 & 4 & 6 & 7 & 21 & 16 & 4 & 112 & 546 & 189 & 137 & 101 & 69 \\
\hline g6pd & 1.106 & 950 & 1.356 & 1.453 & 977 & 818 & 373 & 563 & 485 & 333 & 323 & 330 \\
\hline tubb2b (N-tubulin) & 49 & 55 & 63 & 90 & 91 & 27 & 14.184 & 32.903 & 161 & 117 & 157 & 105 \\
\hline vGlut1 & 33 & 42 & 56 & 63 & 41 & 26 & 111 & 3.024 & 2 & 3 & 1 & 2 \\
\hline NEG_A & 2 & 1 & 7 & 4 & 2 & 1 & 12 & 8 & 5 & 4 & 4 & 2 \\
\hline NEG_B & 6 & 6 & 7 & 7 & 1 & 2 & 6 & 6 & 5 & 5 & 3 & 4 \\
\hline NEG_C & 3 & 3 & 2 & 1 & 4 & 1 & 6 & 4 & 2 & 1 & 6 & 3 \\
\hline NEG_D & 3 & 6 & 9 & 6 & 2 & 1 & 8 & 11 & 9 & 3 & 5 & 6 \\
\hline NEG_E & 5 & 4 & 1 & 5 & 6 & 1 & 6 & 7 & 5 & 4 & 5 & 3 \\
\hline NEG_F & 6 & 15 & 15 & 6 & 8 & 4 & 11 & 13 & 11 & 10 & 13 & 6 \\
\hline NEG_G & 12 & 7 & 11 & 8 & 10 & 9 & 5 & 5 & 5 & 5 & 2 & 2 \\
\hline NEG_H & 4 & 8 & 4 & 7 & 6 & 3 & 5 & 7 & 3 & 2 & 1 & 1 \\
\hline POS_A & 11.995 & 10.430 & 11.453 & 11.338 & 10.769 & 4.119 & 14.514 & 14.988 & 15.429 & 15.767 & 12.424 & 8.790 \\
\hline POS_B & 5.902 & 5.418 & 5.365 & 5.745 & 5.295 & 2.138 & 6.762 & 7.119 & 7.493 & 7.493 & 6.351 & 4.420 \\
\hline POS_C & 1.631 & 1.518 & 1.529 & 1.566 & 1.476 & 587 & 1.882 & 2.000 & 2.120 & 1.997 & 1.796 & 1.176 \\
\hline POS_D & 395 & 326 & 377 & 340 & 346 & 144 & 437 & 411 & 471 & 482 & 431 & 277 \\
\hline POS_E & 64 & 56 & 66 & 48 & 68 & 25 & 92 & 73 & 97 & 93 & 69 & 48 \\
\hline POS_F & 40 & 29 & 34 & 32 & 36 & 10 & 52 & 64 & 50 & 48 & 44 & 31 \\
\hline
\end{tabular}




\subsubsection{Experiment 3.7}

Table 6.11 Primary data of the first Nanostring experiment

\begin{tabular}{|c|c|c|c|c|}
\hline Genes & Ptf1a & Ngn2 & chimeric Ptf1a & CC \\
\hline Arx-a & 17 & 5 & 29 & 12 \\
\hline Ascl1/Xash1 & 17 & 7 & 16 & 6 \\
\hline Cdknx (p27Xic1) & 1.201 & 482 & 950 & 320 \\
\hline DII1 (Delta1) & 623 & 242 & 548 & 74 \\
\hline Delta2 & 591 & 111 & 547 & 22 \\
\hline ESR10 & 92 & 74 & 50 & 3 \\
\hline ESR6e (Hes3.1) & 208 & 201 & 208 & 91 \\
\hline ESR9.1a & 61 & 24 & 37 & 1 \\
\hline Ebf2-a/b & 1.157 & 238 & 726 & 1 \\
\hline Ebf3 (Coe3) & 1.890 & 615 & 1.123 & 12 \\
\hline Eng1-a/b & 2 & 2 & 1 & 4 \\
\hline FGF8 & 61 & 17 & 36 & 7 \\
\hline FoxD3a (XfD6) & 313 & 58 & 199 & 60 \\
\hline Gad1 & 1.078 & 7 & 335 & 3 \\
\hline H4 & 27 & 9 & 29 & 3 \\
\hline $\mathrm{Hb} 9 / \mathrm{mnx} 1$ & 34 & 9 & 26 & 8 \\
\hline Hes1a(Hairy1) & 668 & 453 & 1.160 & 361 \\
\hline Hes2 & 1 & 6 & 6 & 4 \\
\hline Hes5.1 (ESR1) & 149 & 53 & 87 & 22 \\
\hline Hes6.1 & 155 & 59 & 140 & 77 \\
\hline Hes7 (XHR1) & 39 & 21 & 53 & 26 \\
\hline Hox11L2/TIx3 & 22 & 334 & 234 & 9 \\
\hline Hpx6 (Lbx1) & 79 & 29 & 38 & 23 \\
\hline Insm1 & 390 & 186 & 307 & 20 \\
\hline Islet1 (is|1) & 301 & 498 & 537 & 302 \\
\hline Jagged1 (Serrate-1) & 491 & 193 & 402 & 107 \\
\hline Krox20/egr2 & 5 & 6 & 4 & 3 \\
\hline Lhx1 (Lim1) & 95 & 3 & 9 & 1 \\
\hline Lhx2 & 11 & 4 & 6 & 2 \\
\hline Lhx5 & 73 & 4 & 35 & 3 \\
\hline Lmx1b & 105 & 8 & 25 & 4 \\
\hline Mxi1 & 351 & 346 & 416 & 141 \\
\hline MyT1 & 881 & 467 & 970 & 72 \\
\hline NCAMa/b & 1.001 & 988 & 1.344 & 22 \\
\hline NeuroD1a/b & 621 & 312 & 759 & 24 \\
\hline NeuroD4 (Xath3) & 638 & 728 & 1.498 & 3 \\
\hline NeuroD6 (Xath2) & 3 & 3 & 3 & 1 \\
\hline Ngn1 & 195 & 194 & 452 & 5 \\
\hline Ngn3 & 21 & 17 & 27 & 12 \\
\hline Ngnr-1a (ngn2a) & 6 & 2 & 2 & 2 \\
\hline Nkx6.1 & 80 & 5 & 15 & 1 \\
\hline Nkx6.2 & 54 & 33 & 63 & 26 \\
\hline Notch1 & 1.033 & 429 & 910 & 433 \\
\hline Nrgn-1b (Ngn2-b) & 41 & 8 & 18 & 2 \\
\hline Nrp1 & 106 & 40 & 75 & 19 \\
\hline Numb & 3 & 5 & 5 & 3 \\
\hline Numbl & 600 & 389 & 938 & 575 \\
\hline ODC & 14.005 & 7.860 & 12.492 & 6.763 \\
\hline Olig3 & 7 & 5 & 8 & 7 \\
\hline Pak3 & 404 & 291 & 331 & 23 \\
\hline Pax2-a & 149 & 10 & 9 & 4 \\
\hline Pax3 & 147 & 8 & 29 & 1 \\
\hline Pax6-a & 63 & 16 & 37 & 26 \\
\hline Pax6-b & 64 & 6 & 5 & 9 \\
\hline PRDM14 & 78 & 338 & 168 & 13 \\
\hline Ptf1a-a/b & 84 & 3 & 125 & 5 \\
\hline RBM38/Seb4R & 76 & 79 & 110 & 53 \\
\hline RBPJ & 404 & 217 & 531 & 289 \\
\hline Slug & 30 & 8 & 25 & 3 \\
\hline Sox1 & 78 & 26 & 76 & 35 \\
\hline Sox2 & 1.023 & 103 & 182 & 37 \\
\hline Sox3 & 350 & 72 & 107 & 75 \\
\hline Sox9-a/b & 192 & 23 & 37 & 12 \\
\hline SoxD & 2.093 & 999 & 2.055 & 1.299 \\
\hline Twist1-a & 97 & 13 & 22 & 5 \\
\hline Xbra-a/b & 45 & 63 & 101 & 50 \\
\hline actb & 309.705 & 177.958 & 419.321 & 178.041 \\
\hline actc1 & 1.984 & 1.015 & 3.163 & 280 \\
\hline c-myc & 159 & 105 & 229 & 153 \\
\hline g6pd & 470 & 229 & 507 & 159 \\
\hline gapdh & 398 & 312 & 315 & 149 \\
\hline runx1 (Xaml) & 52 & 60 & 92 & 81 \\
\hline tubb2b (N-tubulin) & 6.434 & 3.213 & 4.552 & 39 \\
\hline
\end{tabular}




\begin{tabular}{|l|r|r|r|r|} 
vGlut1 & 72 & 179 & 192 & 12 \\
\hline NEG_A & 3 & 2 & 2 & 2 \\
\hline NEG_B & 3 & 5 & 5 & 5 \\
\hline NEG_C & 3 & 3 & 1 & 2 \\
\hline NEG_D & 3 & 1 & 5 & 3 \\
\hline NEG_E & 6 & 2 & 3 & 5 \\
\hline NEG_F & 6 & 7 & 7 & 6 \\
\hline NEG_G & 12 & 4 & 7 & 6 \\
\hline NEG_H & 14 & 13 & 16 & 7 \\
\hline POS_A & 13.666 & 12.441 & 11.328 & 13.077 \\
\hline POS_B & 7.112 & 6.564 & 6.221 & 6.748 \\
\hline POS_C & 2.392 & 2.164 & 2.052 & 2.172 \\
\hline POS_D & 428 & 368 & 368 & 394 \\
\hline POS_E & 96 & 86 & 74 & 80 \\
\hline POS_F & 30 & 38 & 20 & 39 \\
\hline
\end{tabular}


Table 6.12 Primary data of the second Nanostring experiment

\begin{tabular}{|c|c|c|c|c|}
\hline Genes & Ptf1a & Ngn2 & chimeric Ptf1a & CC \\
\hline Arx-a & 7 & 13 & 13 & 4 \\
\hline Ascl1/Xash1 & 8 & 8 & 13 & 7 \\
\hline Cdknx (p27Xic1) & 1.200 & 885 & 1.084 & 262 \\
\hline DII1 (Delta1) & 1.264 & 516 & 583 & 52 \\
\hline Delta2 & 689 & 263 & 489 & 9 \\
\hline ESR10 & 194 & 104 & 100 & 3 \\
\hline ESR6e (Hes3.1) & 135 & 160 & 162 & 65 \\
\hline ESR9.1a & 208 & 61 & 58 & 3 \\
\hline Ebf2-a/b & 1.341 & 470 & 775 & 1 \\
\hline Ebf3 (Coe3) & 1.414 & 1.207 & 1.079 & 8 \\
\hline Eng1-a/b & 2 & 2 & 3 & 1 \\
\hline FGF8 & 31 & 18 & 21 & 2 \\
\hline FoxD3a (XfD6) & 243 & 106 & 249 & 46 \\
\hline Gad1 & 822 & 10 & 333 & 4 \\
\hline H4 & 2 & 3 & 2 & 2 \\
\hline $\mathrm{Hb9/mnx1}$ & 18 & 32 & 34 & 6 \\
\hline Hes1a(Hairy1) & 457 & 718 & 948 & 277 \\
\hline Hes2 & 23 & 1 & 6 & 2 \\
\hline Hes5.1 (ESR1) & 484 & 99 & 141 & 25 \\
\hline Hes6.1 & 192 & 140 & 160 & 73 \\
\hline Hes7 (XHR1) & 17 & 24 & 26 & 17 \\
\hline Hox11L2/TIx3 & 12 & 783 & 106 & 2 \\
\hline Hpx6 (Lbx1) & 115 & 23 & 34 & 6 \\
\hline Insm1 & 431 & 436 & 243 & 15 \\
\hline Islet1 (is|1) & 69 & 786 & 400 & 186 \\
\hline Jagged1 (Serrate-1) & 372 & 315 & 360 & 94 \\
\hline Krox20/egr2 & 6 & 3 & 9 & 4 \\
\hline Lhx1 (Lim1) & 201 & 18 & 40 & 1 \\
\hline Lhx2 & 13 & 5 & 4 & 2 \\
\hline Lhx5 & 190 & 10 & 45 & 1 \\
\hline Lmx1b & 193 & 31 & 38 & 5 \\
\hline Mxi1 & 191 & 233 & 157 & 76 \\
\hline MyT1 & 949 & 709 & 730 & 48 \\
\hline NCAMa/b & 1.212 & 1.950 & 1.288 & 23 \\
\hline NeuroD1a/b & 835 & 1.009 & 1.003 & 17 \\
\hline NeuroD4 (Xath3) & 1.055 & 1.683 & 1.152 & 4 \\
\hline NeuroD6 (Xath2) & 8 & 3 & 3 & 3 \\
\hline Ngn1 & 327 & 395 & 259 & 2 \\
\hline Ngn3 & 14 & 24 & 16 & 6 \\
\hline Ngnr-1a (ngn2a) & 18 & 7 & 4 & 1 \\
\hline Nkx6.1 & 40 & 8 & 59 & 3 \\
\hline Nkx6.2 & 48 & 58 & 92 & 29 \\
\hline Notch1 & 1.179 & 555 & 820 & 252 \\
\hline Nrgn-1b (Ngn2-b) & 148 & 24 & 22 & 1 \\
\hline Nrp1 & 113 & 128 & 173 & 20 \\
\hline Numb & 9 & 5 & 5 & 3 \\
\hline Numbl & 214 & 600 & 636 & 466 \\
\hline ODC & 14.140 & 9.967 & 11.421 & 5.580 \\
\hline Olig3 & 3 & 2 & 1 & 7 \\
\hline Pak3 & 339 & 600 & 346 & 13 \\
\hline Pax2-a & 168 & 5 & 25 & 3 \\
\hline Pax3 & 269 & 50 & 81 & 1 \\
\hline Pax6-a & 70 & 29 & 45 & 12 \\
\hline Pax6-b & 71 & 14 & 19 & 5 \\
\hline PRDM14 & 35 & 907 & 138 & 12 \\
\hline Ptf1a-a/b & 84 & 6 & 87 & 4 \\
\hline RBM38/Seb4R & 44 & 100 & 73 & 32 \\
\hline RBPJ & 271 & 342 & 419 & 215 \\
\hline Slug & 57 & 7 & 21 & 1 \\
\hline Sox1 & 45 & 42 & 66 & 23 \\
\hline Sox2 & 1.098 & 197 & 426 & 25 \\
\hline Sox3 & 603 & 114 & 280 & 43 \\
\hline Sox9-a/b & 317 & 21 & 62 & 8 \\
\hline SoxD & 1.367 & 1.288 & 1.832 & 929 \\
\hline Twist1-a & 68 & 44 & 89 & 1 \\
\hline Xbra-a/b & 50 & 118 & 51 & 23 \\
\hline actb & 119.965 & 181.584 & 237.167 & 127.128 \\
\hline actc1 & 276 & 1.051 & 1.147 & 164 \\
\hline c-myc & 76 & 205 & 187 & 126 \\
\hline g6pd & 252 & 254 & 306 & 147 \\
\hline gapdh & 177 & 607 & 392 & 132 \\
\hline runx1 (Xaml) & 38 & 54 & 50 & 41 \\
\hline tubb2b (N-tubulin) & 7.652 & 6.724 & 4.566 & 32 \\
\hline vGlut1 & 22 & 520 & 143 & 7 \\
\hline NEG_A & 3 & 6 & 1 & 3 \\
\hline NEG_B & 5 & 5 & 3 & 1 \\
\hline
\end{tabular}




\begin{tabular}{|l|r|r|r|r|} 
NEG_C & 2 & 4 & 1 & 2 \\
\hline NEG_D & 3 & 1 & 1 & 1 \\
\hline NEG_E & 4 & 1 & 1 & 3 \\
\hline NEG_F & 6 & 8 & 4 & 4 \\
\hline NEG_G & 8 & 11 & 3 & 1 \\
\hline NEG_H & 10 & 14 & 18 & 10 \\
\hline POS_A & 9.701 & 11.659 & 13.983 & 9.955 \\
\hline POS_B & 5.454 & 5.933 & 6.721 & 4.774 \\
\hline POS_C & 1.740 & 1.871 & 2.214 & 1.520 \\
\hline POS_D & 295 & 327 & 342 & 261 \\
\hline POS_E & 81 & 64 & 92 & 64 \\
\hline POS_F & 23 & 26 & 27 & 24 \\
\hline
\end{tabular}




\subsubsection{Experiment 3.8 and 3.20}

Table 6.13 Primary data of the first Nanostring experiment

\begin{tabular}{|c|c|c|c|c|c|c|}
\hline & & $6 \mathrm{~h}$ & & & $32 \mathrm{~h}$ & \\
\hline Genes & Ptf1a & Ptf1a $a^{\text {W224AW242A }}$ & CC & Ptf1a & Ptf1a ${ }^{\text {W224AW242A }}$ & cc \\
\hline Ascl1/Xash1 new & 10 & 20 & 26 & 23 & 34 & 25 \\
\hline Barhl2 & 5 & 3 & 1 & 106 & 30 & 3 \\
\hline Cdknx (p27Xic1) & 784 & 701 & 93 & 1.192 & 1.368 & 304 \\
\hline Cpa1 & 2 & 1 & 1 & 13 & 5 & 5 \\
\hline DII1 (Delta1) & 3.115 & 4.298 & 181 & 5.389 & 3.345 & 571 \\
\hline ESR10 & 624 & 64 & 66 & 823 & 828 & 80 \\
\hline Ebf2-a/b & 296 & 90 & 3 & 2.980 & 2.509 & 1 \\
\hline Ebf3 (Coe3) & 5 & 5 & 1 & 982 & 1.700 & 30 \\
\hline FoxD3a (XfD6) & 18 & 59 & 25 & 134 & 46 & 1 \\
\hline Gad1 & 3 & 2 & 1 & 1.097 & 40 & 2 \\
\hline H4 new & 133.016 & 143.976 & 123.942 & 36.571 & 45.747 & 47.895 \\
\hline HNF6 gene2 & 25 & 16 & 11 & 4.867 & 2.949 & 5 \\
\hline Hb9/mnx1 & 8 & 28 & 12 & 7 & 33 & 2 \\
\hline Hes5.1 (ESR1) & 982 & 166 & 22 & 755 & 730 & 8 \\
\hline $\mathrm{Hmx3}$ & 6 & 8 & 3 & 50 & 7 & 5 \\
\hline Hox11L2/TIx3 & 15 & 33 & 1 & 17 & 760 & 10 \\
\hline Hpx6 (Lbx1) & 3 & 1 & 4 & 247 & 202 & 2 \\
\hline Islet1 (is|1) & 2 & 2 & 1 & 97 & 289 & 241 \\
\hline Kirrel2 & 229 & 67 & 94 & 1.122 & 41 & 15 \\
\hline Lhx1 (Lim1) & 12 & 22 & 1 & 209 & 98 & 2 \\
\hline Lhx2 & 3 & 3 & 8 & 41 & 35 & 6 \\
\hline Lhx5 & 305 & 613 & 577 & 517 & 14 & 2 \\
\hline Lmx1b & 20 & 5 & 8 & 106 & 120 & 2 \\
\hline Mecom & 3 & 4 & 1 & 116 & 13 & 7 \\
\hline Mxi1 & 1.094 & 998 & 181 & 625 & 847 & 578 \\
\hline MyT1 & 2.229 & 1.574 & 66 & 2.718 & 2.948 & 274 \\
\hline NCAMa/b & 25 & 28 & 21 & 2.335 & 3.279 & 73 \\
\hline NeuroD1a/b & 3 & 6 & 2 & 1.166 & 1.165 & 1 \\
\hline NeuroD4 (Xath3) & 14 & 11 & 1 & 1.173 & 2.258 & 2 \\
\hline Ngn1 & 1 & 2 & 4 & 455 & 771 & 3 \\
\hline Ngn3 & 2 & 19 & 5 & 1 & 4 & 1 \\
\hline Nrgn-1b (Ngn2-b) & 26 & 47 & 43 & 160 & 21 & 1 \\
\hline ODC & 11.580 & 13.401 & 11.191 & 14.778 & 16.784 & 11.418 \\
\hline PRDM13 & 936 & 55 & 1 & 3.328 & 186 & 1 \\
\hline PRDM14 & 85 & 398 & 102 & 291 & 1.903 & 6 \\
\hline Pak3 & 98 & 103 & 65 & 746 & 978 & 7 \\
\hline Pax2-a & 10 & 8 & 9 & 1.065 & 59 & 28 \\
\hline Ptf1a-a/b 5'UTR & 22 & 33 & 17 & 42 & 15 & 9 \\
\hline RBM38/Seb4R & 893 & 1.141 & 542 & 222 & 203 & 175 \\
\hline RBPJ 3'UTR & 728 & 781 & 731 & 566 & 512 & 468 \\
\hline Sox3 & 4.238 & 7.499 & 5.825 & 812 & 554 & 152 \\
\hline Sox9-a/b & 108 & 116 & 63 & 456 & 290 & 10 \\
\hline XPDip & 3 & 2 & 2 & 19 & 2 & 1 \\
\hline Xbra-a/b & 32 & 21 & 25 & 103 & 20 & 166 \\
\hline actc1 & 13 & 21 & 12 & 734 & 810 & 205 \\
\hline g6pd & 503 & 596 & 488 & 184 & 180 & 193 \\
\hline tubb2b (N-tubulin) & 51 & 41 & 25 & 18.125 & 33.288 & 58 \\
\hline vGlut1 & 1 & 1 & 1 & 34 & 597 & 1 \\
\hline NEG_A & 2 & 7 & 5 & 3 & 3 & 1 \\
\hline NEG_B & 2 & 1 & 1 & 1 & 1 & 6 \\
\hline NEG_C & 3 & 1 & 1 & 1 & 2 & 1 \\
\hline NEG_D & 6 & 6 & 6 & 6 & 7 & 3 \\
\hline NEG_E & 9 & 8 & 3 & 4 & 2 & 5 \\
\hline NEG_F & 1 & 9 & 3 & 6 & 5 & 2 \\
\hline NEG_G & 1 & 1 & 2 & 1 & 2 & 1 \\
\hline NEG_H & 2 & 1 & 1 & 2 & 1 & 1 \\
\hline POS_A & 5.392 & 6.917 & 6.116 & 6.147 & 7.891 & 7.303 \\
\hline POS_B & 2.813 & 3.382 & 3.010 & 3.226 & 3.900 & 3.563 \\
\hline POS_C & 864 & 1.047 & 892 & 1.010 & 1.192 & 1.069 \\
\hline POS_D & 175 & 226 & 198 & 206 & 242 & 203 \\
\hline POS_E & 24 & 30 & 30 & 38 & 45 & 34 \\
\hline POS_F & 33 & 32 & 29 & 20 & 30 & 28 \\
\hline
\end{tabular}


Table 6.14 Primary data of the second Nanostring experiment

\begin{tabular}{|c|c|c|c|c|c|c|}
\hline 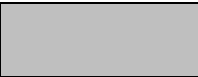 & 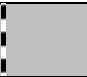 & $6 \mathrm{~h}$ & 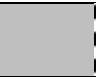 & 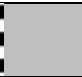 & $32 \mathrm{~h}$ & \\
\hline Genes & Ptf1a & Ptf1a $a^{\text {W224AW242A }}$ & CC & Ptf1a & Ptf1a $a^{\text {W224AW242A }}$ & CC \\
\hline Ascl1/Xash1 new & 16 & 16 & 21 & 12 & 36 & 7 \\
\hline Barhl2 & 1 & 1 & 3 & 16 & 14 & 3 \\
\hline Cdknx (p27Xic1) & 277 & 377 & 146 & 459 & 1.502 & 141 \\
\hline Cpa1 & 1 & 1 & 3 & 2 & 51 & 3 \\
\hline DII1 (Delta1) & 688 & 1.113 & 96 & 615 & 2.060 & 51 \\
\hline ESR10 & 241 & 53 & 86 & 61 & 283 & 7 \\
\hline Ebf2-a/b & 91 & 33 & 6 & 538 & 1.564 & 1 \\
\hline Ebf3 (Coe3) & 1 & 1 & 3 & 206 & 1.962 & 3 \\
\hline FoxD3a (XfD6) & 64 & 93 & 162 & 45 & 40 & 1 \\
\hline Gad1 & 1 & 1 & 1 & 275 & 96 & 1 \\
\hline H4 new & 98.113 & 124.321 & 239.505 & 24.893 & 63.752 & 16.260 \\
\hline HNF6 gene2 & 22 & 20 & 11 & 1.028 & 1.981 & 3 \\
\hline Hb9/mnx1 & 19 & 26 & 26 & 7 & 18 & 3 \\
\hline Hes5.1 (ESR1) & 174 & 115 & 55 & 59 & 200 & 1 \\
\hline $\mathrm{Hmx3}$ & 7 & 3 & 6 & 15 & 12 & 4 \\
\hline Hox11L2/TIx3 & 5 & 13 & 5 & 12 & 832 & 1 \\
\hline Hpx6 (Lbx1) & 4 & 2 & 5 & 63 & 52 & 1 \\
\hline Islet1 (isl1) & 1 & 2 & 2 & 130 & 897 & 75 \\
\hline Kirrel2 & 145 & 27 & 118 & 97 & 31 & 4 \\
\hline Lhx1 (Lim1) & 14 & 17 & 17 & 52 & 43 & 1 \\
\hline Lhx2 & 3 & 4 & 5 & 7 & 23 & 10 \\
\hline Lhx5 & 290 & 223 & 826 & 31 & 10 & 1 \\
\hline Lmx1b & 6 & 8 & 11 & 16 & 73 & 1 \\
\hline Mecom & 2 & 2 & 4 & 42 & 40 & 1 \\
\hline Mxi1 & 945 & 871 & 718 & 207 & 813 & 145 \\
\hline MyT1 & 952 & 523 & 150 & 589 & 3.503 & 113 \\
\hline NCAMa/b & 19 & 27 & 40 & 855 & 6.549 & 52 \\
\hline NeuroD1a/b & 2 & 4 & 7 & 193 & 1.218 & 4 \\
\hline NeuroD4 (Xath3) & 4 & 1 & 2 & 160 & 1.655 & 1 \\
\hline Ngn1 & 1 & 5 & 6 & 108 & 761 & 2 \\
\hline Ngn3 & 13 & 4 & 11 & 1 & 4 & 1 \\
\hline Nrgn-1b (Ngn2-b) & 60 & 72 & 67 & 29 & 25 & 1 \\
\hline ODC & 6.931 & 10.663 & 17.736 & 3.435 & 27.572 & 1.312 \\
\hline PRDM13 & 347 & 19 & 1 & 179 & 46 & 1 \\
\hline PRDM14 & 218 & 182 & 266 & 149 & 2.322 & 4 \\
\hline Pak3 & 49 & 52 & 72 & 176 & 1.157 & 3 \\
\hline Pax2-a & 12 & 8 & 8 & 97 & 127 & 8 \\
\hline Ptf1a-a/b 5'UTR & 20 & 20 & 19 & 21 & 56 & 6 \\
\hline RBM38/Seb4R & 567 & 995 & 871 & 66 & 311 & 40 \\
\hline RBPJ 3'UTR & 452 & 460 & 757 & 344 & 1.661 & 166 \\
\hline Sox3 & 5.523 & 7.875 & 11.863 & 242 & 255 & 58 \\
\hline Sox9-a/b & 112 & 68 & 116 & 151 & 134 & 4 \\
\hline Pdia2 (XPDip) & 4 & 2 & 3 & 1 & 4 & 1 \\
\hline Xbra-a/b & 12 & 29 & 26 & 31 & 288 & 10 \\
\hline actc1 & 12 & 9 & 14 & 254 & 670 & 28 \\
\hline g6pd & 310 & 479 & 854 & 65 & 670 & 31 \\
\hline tubb2b (N-tubulin) & 7 & 4 & 14 & 2.905 & 36.739 & 21 \\
\hline vGlut1 & 1 & 1 & 1 & 9 & 1.485 & 1 \\
\hline NEG_A & 3 & 6 & 11 & 2 & 5 & 6 \\
\hline NEG_B & 4 & 2 & 7 & 2 & 3 & 3 \\
\hline NEG_C & 4 & 3 & 1 & 2 & 2 & 1 \\
\hline NEG_D & 3 & 5 & 6 & 5 & 16 & 1 \\
\hline NEG_E & 2 & 7 & 9 & 3 & 5 & 6 \\
\hline NEG_F & 6 & 8 & 6 & 5 & 5 & 5 \\
\hline NEG_G & 1 & 2 & 3 & 1 & 3 & 1 \\
\hline NEG_H & 2 & 2 & 3 & 1 & 2 & 1 \\
\hline POS_A & 9.006 & 9.492 & 7.183 & 9.700 & 7.705 & 6.893 \\
\hline POS_B & 4.727 & 4.675 & 3.742 & 4.913 & 4.638 & 3.554 \\
\hline POS_C & 1.321 & 1.372 & 1.123 & 1.477 & 1.439 & 1.005 \\
\hline POS_D & 291 & 306 & 242 & 340 & 325 & 222 \\
\hline POS_E & 61 & 64 & 37 & 47 & 49 & 32 \\
\hline POS_F & 38 & 40 & 44 & 37 & 39 & 23 \\
\hline
\end{tabular}




\subsubsection{Experiment 3.10 and 3.21}

Table 6.15 Primary data of the first Nanostring experiment

\begin{tabular}{|c|c|c|c|c|c|c|c|c|c|c|c|c|c|c|c|c|}
\hline , & 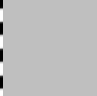 & Ptf1 & & ו & 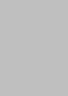 & Ptf1a+s & $\mathrm{Su}(\mathrm{H})$ & 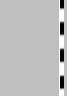 & & $\mathrm{Su}(\mathrm{H}$ & & I & & C & C & \\
\hline Genes & $3 \mathrm{~h}$ & $6 \mathrm{~h}$ & $9 \mathrm{~h}$ & $25 \mathrm{~h}$ & $3 \mathrm{~h}$ & $6 \mathrm{~h}$ & $9 \mathrm{~h}$ & $25 \mathrm{~h}$ & $3 \mathrm{~h}$ & $6 \mathrm{~h}$ & $9 \mathrm{~h}$ & $25 \mathrm{~h}$ & $3 \mathrm{~h}$ & $6 \mathrm{~h}$ & $9 \mathrm{~h}$ & $25 \mathrm{~h}$ \\
\hline Ascl1/Xash1 new & 17 & 11 & 14 & 2 & 18 & 18 & 19 & 7 & 10 & 8 & 12 & 1 & 7 & 13 & 14 & 4 \\
\hline Barhl2 & 5 & 1 & 6 & 22 & 2 & 11 & 11 & 94 & 1 & 1 & 1 & 1 & 1 & 1 & 1 & 1 \\
\hline Cdknx (p27Xic1) & 625 & 631 & 584 & 573 & 199 & 353 & 464 & 588 & 56 & 145 & 110 & 27 & 31 & 51 & 139 & 171 \\
\hline Cpa1 & 1 & 2 & 1 & 34 & 1 & 1 & 4 & 61 & 1 & 1 & 1 & 2 & 1 & 1 & 1 & 14 \\
\hline DII1 (Delta1) & 2.458 & 1.819 & 1.889 & 2.509 & 545 & 369 & 952 & 4.082 & 66 & 232 & 192 & 12 & 19 & 74 & 232 & 119 \\
\hline ESR10 & 1.108 & 603 & 1.003 & 337 & 1.175 & 521 & 1.003 & 1.166 & 33 & 55 & 35 & 1 & 21 & 9 & 18 & 1 \\
\hline Ebf2-a/b & 56 & 282 & 329 & \begin{tabular}{|c|}
1.504 \\
\end{tabular} & 10 & 109 & 319 & 1.257 & 5 & 2 & 7 & 1 & 1 & 3 & 3 & 1 \\
\hline Ebf3 (Coe3) & 1 & 2 & 3 & 605 & 1 & 1 & 2 & 285 & 1 & 1 & 1 & 1 & 1 & 1 & 1 & 5 \\
\hline FoxD3a (XfD6) & 13 & 21 & 14 & 168 & 10 & 10 & 27 & 320 & 16 & 8 & 10 & 1 & 21 & 12 & 7 & 1 \\
\hline Gad1 & 1 & 1 & 1 & 554 & 1 & 3 & 14 & 151 & 1 & 1 & 1 & 1 & 1 & 1 & 1 & 5 \\
\hline H4 new & 102.50011 & 11.754 & 127.5843 & 38.854 & 71.736 & 96.5621 & 151.9634 & 46.345 & 86.761 & 119.900 & 75.1165 & 5.829 & 62.650 & 53.195 & 106.944 & 26.719 \\
\hline HNF6 gene2 & 31 & 33 & 66 & 3.336 & 12 & 17 & 40 & 3.248 & 3 & 18 & 18 & 2 & 6 & 1 & 13 & 5 \\
\hline $\mathrm{Hb9/mnx1}$ & 3 & 6 & 7 & 7 & 2 & 6 & 10 & 1 & 3 & 4 & 2 & 1 & 1 & 1 & 4 & 1 \\
\hline Hes5.1 (ESR1) & 1.043 & 1.028 & 1.613 & 352 & 632 & 643 & 1.484 & 519 & 17 & 72 & 37 & 1 & 10 & 7 & 30 & 3 \\
\hline $\mathrm{Hm} \times 3$ & 2 & 6 & 3 & 58 & 2 & 5 & 10 & 14 & 7 & 7 & 4 & 1 & 1 & 1 & 2 & 8 \\
\hline Hox11L2/TIx3 & 5 & 5 & 8 & 3 & 1 & 5 & 1 & 2 & 1 & 2 & 1 & 1 & 1 & 1 & 1 & 1 \\
\hline Hpx6 (Lbx1) & 3 & 1 & 3 & 29 & 1 & 2 & 6 & 51 & 1 & 2 & 2 & 2 & 1 & 2 & 5 & 1 \\
\hline Islet1 (isl1) & 1 & 1 & 2 & 78 & 3 & 1 & 3 & 17 & 1 & 1 & 2 & 32 & 2 & 1 & 1 & 238 \\
\hline Kirrel2 & 243 & 189 & 333 & 1.287 & 82 & 141 & 272 & 2.310 & 107 & 182 & 112 & 1 & 66 & 48 & 168 & 1 \\
\hline Lhx1 (Lim1) & 10 & 5 & 2 & 48 & 4 & 2 & 7 & 184 & 4 & 4 & 1 & 1 & 2 & 2 & 2 & 1 \\
\hline Lhx2 & 3 & 7 & 19 & 8 & 5 & 4 & 20 & 270 & 2 & 4 & 2 & 1 & 1 & 2 & 2 & 1 \\
\hline Lhx5 & 460 & 293 & 336 & 185 & 158 & 122 & 265 & 129 & 83 & 183 & 81 & 1 & 99 & 51 & 183 & 1 \\
\hline Lmx1b & 20 & 17 & 20 & 77 & 11 & 6 & 20 & 57 & 4 & \begin{tabular}{c|c|}
17 \\
\end{tabular} & 3 & 1 & 2 & 3 & 16 & 2 \\
\hline Mecom & 3 & 5 & 8 & 166 & 1 & 4 & 12 & 2.344 & 1 & 1 & 1 & 1 & 1 & 1 & 1 & 9 \\
\hline Mxi1 & 1.041 & 1.204 & 1.674 & 267 & 359 & 496 & 892 & 335 & 164 & 433 & 357 & 22 & 111 & 154 & 384 & 136 \\
\hline MyT1 & 867 & 1.475 & 1.220 & 1.415 & 333 & 893 & 1.474 & 1.892 & 31 & 78 & 50 & 11 & 16 & 34 & 69 & 196 \\
\hline NCAMa/b & 15 & 10 & 6 & 1.864 & 8 & 10 & 9 & 918 & 7 & 14 & 7 & 4 & 7 & 9 & 13 & 46 \\
\hline NeuroD1a/b & 7 & 3 & 11 & 325 & 3 & 2 & 12 & 107 & 2 & 1 & 2 & 1 & 1 & 2 & 2 & 1 \\
\hline NeuroD4 (Xath3) & 4 & 36 & 42 & 635 & 3 & 19 & 35 & 383 & 1 & 1 & 1 & 1 & 1 & 1 & 2 & 1 \\
\hline Ngn1 & 1 & 1 & 1 & 204 & 1 & 1 & 3 & 489 & 2 & 2 & 1 & 1 & 1 & 1 & 5 & 1 \\
\hline Ngn3 & 2 & 9 & 1 & 1 & 3 & 7 & 13 & 1 & 3 & 8 & 2 & 1 & 1 & 2 & 3 & 1 \\
\hline Nrgn-1b (Ngn2-b) & 31 & 42 & 35 & 95 & 24 & 29 & 55 & 382 & 11 & 13 & 16 & 1 & 21 & 25 & 23 & 2 \\
\hline ODC & 12.073 & 9.175 & 12.3091 & 12.691 & 4.193 & 4.615 & $8.175 \mid 1$ & 11.380 & 3.733 & 7.757 & 5.371 & 731 & 3.293 & 2.540 & 6.828 & 7.585 \\
\hline PRDM13 & 158 & 600 & 819 & 2.095 & 177 & 837 & 1.726 & 4.050 & 1 & 1 & 1 & 1 & 1 & 1 & 1 & 1 \\
\hline PRDM14 & 315 & 178 & 214 & 65 & 136 & 88 & 172 & 14 & 79 & 174 & 225 & 1 & 58 & 48 & 189 & 2 \\
\hline Pak3 & 78 & 57 & 72 & 370 & 18 & 34 & 61 & 757 & 18 & 31 & 23 & 2 & 18 & 13 & 28 & 13 \\
\hline Pax2-a & 9 & 6 & 1 & 428 & 3 & 2 & 5 & 192 & 1 & 7 & 4 & 3 & 4 & 2 & 4 & 3 \\
\hline Ptf1a-a/b 5'UTR & 18 & 29 & 34 & 77 & 33 & 51 & 130 & 397 & 10 & 11 & 10 & 14 & 13 & 13 & 14 & 25 \\
\hline RBM38/Seb4R & 702 & 846 & 849 & 144 & 299 & 383 & 556 & 209 & 186 & 294 & 206 & 12 & 279 & 208 & 278 & 148 \\
\hline RBPJ 3'UTR & 766 & 627 & 659 & 737 & 347 & 349 & 536 & 475 & 321 & 507 & 311 & 122 & 332 & 251 & 423 & 544 \\
\hline Sox3 & 7.473 & 5.484 & 6.041 & 235 & 3.140 & 2.854 & 4.181 & 3.311 & 2.925 & 4.034 & 2.765 & \begin{tabular}{|r|}
12 \\
\end{tabular} & 2.749 & 1.862 & 3.984 & 56 \\
\hline Sox9-a/b & 190 & 139 & 183 & 525 & 153 & 165 & 293 & 667 & 48 & 101 & 78 & 1 & 39 & 30 & 68 & 11 \\
\hline Pdia2 (XPDip) & 2 & 9 & 14 & 18 & 4 & 16 & 58 & 878 & 2 & 4 & 1 & 1 & 1 & 1 & 3 & 2 \\
\hline Xbra-a/b & \begin{tabular}{l|l}
4 &
\end{tabular} & 9 & 10 & 13 & 4 & 6 & 10 & 4 & 4 & 2 & 4 & 1 & 4 & 1 & 4 & 25 \\
\hline actc1 & 16 & 25 & 21 & 223 & 11 & 15 & 23 & 106 & 14 & 6 & 3 & 64 & 5 & 5 & 14 & 379 \\
\hline g6pd & 514 & \begin{tabular}{ll|}
458 \\
\end{tabular} & 496 & 214 & 222 & 227 & 424 & 105 & 165 & 339 & 251 & 15 & 183 & 131 & 345 & 186 \\
\hline tubb2b (N-tubulin) & 44 & 37 & 61 & 8.515 & 22 & 26 & 39 & 3.058 & 7 & 13 & 12 & 9 & 5 & 11 & 23 & 101 \\
\hline \begin{tabular}{|l|l} 
vGlut1 \\
\end{tabular} & 9 & 8 & 5 & \begin{tabular}{|l|}
19 \\
\end{tabular} & 1 & 3 & 3 & 4 & 1 & 4 & 1 & 1 & 1 & 1 & 4 & 1 \\
\hline NEG_A & 1 & 4 & 3 & 6 & 2 & 2 & 3 & 1 & 5 & 7 & 5 & 1 & 2 & 1 & 6 & 1 \\
\hline NEG_B & 1 & 3 & 1 & 10 & 1 & 3 & 2 & 3 & 3 & 1 & 1 & 2 & 2 & 6 & 7 & 2 \\
\hline NEG_C & 2 & 1 & 1 & 1 & 1 & 1 & 1 & 3 & 1 & 3 & 1 & 1 & 1 & 1 & 2 & 3 \\
\hline NEG_D & 5 & 6 & 6 & 7 & 3 & 1 & 7 & 7 & 2 & 2 & 7 & 3 & 4 & 5 & 6 & 4 \\
\hline NEG_E & 5 & 8 & 5 & 3 & 7 & 7 & 8 & 2 & 4 & 6 & 7 & 1 & 5 & 3 & 7 & 3 \\
\hline NEG_F & 6 & 4 & 3 & 11 & 4 & 3 & 5 & 5 & 1 & 4 & 4 & 7 & 2 & 7 & 5 & 4 \\
\hline NEG_G & 1 & 1 & 2 & 1 & 1 & 1 & 2 & 1 & 2 & 1 & 1 & 1 & 1 & 1 & 1 & 1 \\
\hline NEG_H & 4 & 2 & 5 & 1 & 1 & 1 & 4 & 1 & 1 & 3 & 1 & 1 & 1 & 1 & 1 & 1 \\
\hline POS_A & 133 & 6.816 & 5.679 & 7.231 & 5.288 & 5.724 & 6.930 & 7.843 & 7.713 & 7.231 & 6.7495 & 5.695 & 6.402 & 5.512 & 9.721 & 7.005 \\
\hline POS_B & 3.066 & 3.436 & 2.752 & \begin{tabular}{|c|}
3.688 \\
\end{tabular} & 2.446 & 2.867 & 3.274 & 3.615 & 3.536 & 3.379 & 3.1492 & \begin{tabular}{|l|}
2.612 \\
\end{tabular} & 3.308 & 2.870 & 5.089 & 3.714 \\
\hline POS_C & 927 & 1.026 & 797 & 1.143 & 765 & 842 & 936 & 1.083 & $\begin{array}{ll}1.017 \\
\end{array}$ & 1.066 & 901 & \begin{tabular}{|l|}
802 \\
\end{tabular} & 1.029 & 850 & 1.502 & 1.150 \\
\hline POS_D & 188 & \begin{tabular}{l|}
238 \\
\end{tabular} & 199 & 220 & 166 & $\begin{array}{ll}198 \\
\end{array}$ & 180 & $\begin{array}{r}257 \\
\end{array}$ & 236 & \begin{tabular}{r|}
214 \\
\end{tabular} & 198 & \begin{tabular}{|l|}
164 \\
\end{tabular} & \begin{tabular}{|l|}
233 \\
\end{tabular} & \begin{tabular}{l|l|}
189 \\
\end{tabular} & 344 & 243 \\
\hline POS_E & 35 & 44 & 28 & 43 & 33 & 26 & 39 & 38 & 38 & 29 & 47 & 29 & 40 & 33 & 66 & 54 \\
\hline POS_F & 35 & 36 & 37 & 35 & 27 & 34 & 42 & 28 & 24 & 33 & 27 & 10 & 30 & 26 & 39 & 27 \\
\hline
\end{tabular}


Table 6.16 Primary data of the second Nanostring experiment

\begin{tabular}{|c|c|c|c|c|c|c|c|c|c|c|c|c|c|c|c|c|}
\hline \multirow[b]{2}{*}{ Genes } & \multicolumn{4}{|c|}{ Ptf1a } & \multicolumn{4}{|c|}{$\mathrm{Ptf1} a+\mathrm{Su}(\mathrm{H})$} & \multicolumn{4}{|c|}{$\mathrm{Su}(\mathrm{H})$} & \multicolumn{4}{|c|}{ CC } \\
\hline & $3 \mathrm{~h}$ & $6 \mathrm{~h}$ & $9 \mathrm{~h}$ & $25 \mathrm{~h}$ & $3 \mathrm{~h}$ & $6 \mathrm{~h}$ & $9 \mathrm{~h}$ & $25 \mathrm{~h}$ & $3 \mathrm{~h}$ & $6 \mathrm{~h}$ & $9 \mathrm{~h}$ & $25 \mathrm{~h}$ & $3 \mathrm{~h}$ & $6 \mathrm{~h}$ & $9 \mathrm{~h}$ & $25 \mathrm{~h}$ \\
\hline Ascl1/Xash1 new & 28 & 24 & 12 & 5 & 25 & 20 & 25 & 2 & 19 & 14 & 13 & 11 & 18 & 28 & 15 & 3 \\
\hline Barhl2 & 1 & 2 & 1 & 25 & 13 & 8 & 62 & 70 & 1 & 1 & 1 & 1 & 1 & 3 & 3 & 2 \\
\hline Cdknx (p27Xic1) & 605 & 845 & 624 & 923 & 462 & 541 & 1.112 & 575 & 97 & 153 & 153 & 363 & 118 & 214 & 262 & 388 \\
\hline Cpa1 & 1 & 1 & 5 & 8 & 1 & 2 & 7 & 21 & 1 & 1 & 1 & 7 & 1 & 1 & 1 & 10 \\
\hline DII1 (Delta1) & 2.410 & 1.843 & 1.649 & 3.065 & 825 & 406 & 1.630 & 3.120 & 126 & 140 & 327 & 157 & 89 & 135 & 774 & 144 \\
\hline ESR10 & 1.558 & 630 & 887 & 911 & 2.621 & 452 & 1.577 & 1.379 & 51 & 134 & 98 & 8 & 57 & 124 & 92 & 3 \\
\hline Ebf2-a/b & 35 & 195 & 286 & 1.673 & 13 & 82 & 613 & 1.050 & 5 & 3 & 7 & 1 & 6 & 5 & 5 & 1 \\
\hline Ebf3 (Coe3) & 6 & 3 & 7 & 690 & 1 & 2 & 5 & 254 & 1 & 1 & 1 & 5 & 1 & 1 & 1 & 7 \\
\hline FoxD3a (XfD6) & 22 & 22 & 31 & 219 & 37 & 31 & 73 & 350 & 36 & 51 & 20 & 1 & 18 & 48 & 47 & 1 \\
\hline Gad1 & 1 & 1 & 2 & 472 & 3 & 19 & 99 & 163 & 1 & 1 & 1 & 4 & 1 & 3 & 2 & 1 \\
\hline H4 new & 124.506 & 210.768 & 195.625 & 57.345 & 155.480 & 158.466 & 254.992 & 62.736 & 168.362 & 129.976 & 99.010 & 38.717 & 147.306 & 176.711 & 177.596 & 50.263 \\
\hline HNF6 gene2 & 8 & 21 & 60 & 3.640 & 9 & 31 & $\begin{array}{r}87 \\
\end{array}$ & 2.695 & 10 & 7 & 10 & 7 & 6 & 11 & 31 & 11 \\
\hline Hb9/mnx1 & 16 & 9 & 8 & 1 & 21 & 29 & 40 & 1 & 11 & 3 & 3 & 1 & 9 & 9 & 13 & 3 \\
\hline Hes5.1 (ESR1) & 1.548 & 1.148 & 1.470 & 410 & 1.821 & 1.310 & 4.196 & 400 & 30 & 59 & 51 & 15 & 22 & 42 & 66 & 3 \\
\hline $\mathrm{Hm} \times 3$ & 4 & 4 & 8 & 67 & 8 & 6 & 12 & 8 & 8 & 3 & 5 & 5 & 9 & 3 & 7 & 4 \\
\hline Hox11L2/TIx3 & 5 & 8 & 11 & 2 & 4 & 2 & 6 & 5 & 2 & 1 & 1 & 3 & 5 & 4 & 6 & 2 \\
\hline Hpx6 (Lbx1) & 5 & 5 & 4 & 9 & 4 & 6 & 6 & 13 & 3 & 1 & 3 & 2 & 1 & 4 & 9 & 1 \\
\hline Islet1 (is|1) & 1 & 5 & 4 & 70 & 2 & 1 & 4 & 31 & 2 & 1 & 1 & 167 & 1 & 2 & 3 & 226 \\
\hline Kirrel2 & 106 & 177 & 307 & 1.461 & 128 & 112 & 647 & 2.255 & 116 & 108 & 138 & 1 & 218 & 126 & 331 & 3 \\
\hline Lhx1 (Lim1) & 8 & 7 & 6 & 46 & 6 & 9 & 14 & 141 & 6 & 3 & 3 & 2 & 3 & 2 & 3 & 1 \\
\hline Lhx2 & 4 & 7 & 16 & 14 & 5 & 5 & 41 & 245 & 1 & 6 & 3 & 1 & 5 & 2 & 3 & 2 \\
\hline Lhx5 & 353 & 304 & 350 & 148 & 398 & 238 & 507 & 130 & 208 & 169 & 101 & 1 & 321 & 387 & 404 & 1 \\
\hline Lmx1b & 10 & 12 & 5 & 24 & 15 & 9 & 15 & 31 & 12 & 8 & 5 & 3 & 13 & 8 & 15 & 1 \\
\hline Mecom & 5 & 4 & 3 & 187 & 2 & 6 & 31 & 2.680 & 2 & 3 & 1 & 8 & 3 & 1 & 1 & 5 \\
\hline Mxi1 & 734 & 1.506 & 1.887 & 314 & 408 & 637 & 2.158 & 338 & 223 & 376 & 508 & 204 & 209 & 333 & 897 & 230 \\
\hline MyT1 & 459 & 1.418 & 1.398 & 1.131 & 358 & 1.059 & 3.655 & 1.562 & 35 & 74 & 67 & 210 & 76 & 106 & 198 & 289 \\
\hline NCAMa/b & 27 & 27 & 38 & 1.953 & 14 & 16 & 40 & 593 & 20 & 21 & 19 & 75 & 19 & 18 & 27 & 37 \\
\hline NeuroD1a/b & 1 & 1 & 9 & 529 & 6 & 2 & 17 & 90 & 1 & 2 & 2 & 1 & 1 & 5 & 1 & 1 \\
\hline NeuroD4 (Xath3) & 1 & 9 & 8 & 1.053 & 1 & 4 & 36 & 291 & 1 & 1 & 1 & 1 & 1 & 1 & 1 & 1 \\
\hline Ngn1 & 10 & 3 & 1 & 330 & 5 & 3 & 3 & 430 & 4 & 5 & 2 & 1 & 3 & 12 & 2 & 1 \\
\hline Ngn3 & 10 & 11 & 6 & 2 & 9 & 7 & 10 & 1 & 12 & 2 & 2 & 1 & 2 & 8 & 5 & 1 \\
\hline Nrgn-1b (Ngn2-b) & 59 & 51 & 57 & 108 & 99 & 67 & 154 & 287 & 48 & 42 & 14 & 1 & 82 & 82 & 41 & 1 \\
\hline ODC & 8.015 & 8.980 & 9.436 & 10.650 & 7.993 & 6.083 & 14.490 & 7.827 & 6.634 & 5.686 & 5.023 & 5.584 & 9.766 & 9.077 & 13.658 & 7.115 \\
\hline PRDM13 & 152 & 901 & 1.157 & 2.463 & 398 & 1.189 & 4.529 & 3.774 & 1 & 1 & 1 & 1 & 2 & 1 & 1 & 1 \\
\hline PRDM14 & 385 & 300 & 448 & 57 & 376 & 247 & 589 & 22 & 251 & 556 & 598 & 11 & 200 & 325 & 668 & 2 \\
\hline Pak3 & 82 & 72 & 89 & 397 & 57 & 66 & 155 & 598 & 46 & 50 & 31 & 4 & 92 & 88 & 96 & 8 \\
\hline Pax2-a & 5 & 7 & 11 & 402 & 7 & 7 & 5 & 94 & 8 & 5 & 2 & 21 & 5 & 4 & 7 & 5 \\
\hline Ptf1a-a/b 5'UTR & 17 & 44 & 33 & 117 & 63 & 99 & 374 & 477 & 16 & 10 & 12 & 28 & 12 & 22 & 13 & 49 \\
\hline RBM38/Seb4R & 603 & 771 & 645 & 152 & 479 & 464 & 928 & 203 & 278 & 223 & 164 & 77 & 436 & 509 & 424 & 156 \\
\hline RBPJ 3'UTR & 962 & 878 & 770 & 631 & 1.059 & 728 & 1.250 & 382 & 707 & 626 & 368 & 392 & 1.040 & 980 & 1.018 & 500 \\
\hline Sox3 & 6.726 & 6.927 & 5.455 & 289 & 7.008 & 5.108 & 8.027 & 3.583 & 5.502 & 5.078 & 2.291 & 62 & 7.427 & 8.248 & 7.028 & 95 \\
\hline Sox9-a/b & 110 & 110 & 127 & 376 & 164 & 255 & 644 & 812 & 39 & 60 & 60 & 19 & 61 & 60 & 107 & 13 \\
\hline Pdia2 (XPDip) & 5 & 10 & 25 & 15 & 8 & 22 & 253 & 888 & 1 & 1 & 1 & 1 & 2 & 1 & 4 & 3 \\
\hline Xbra-a/b & 63 & 4 & 14 & 10 & 17 & 5 & 37 & 2 & 4 & 1 & 2 & 8 & 9 & 3 & 8 & 20 \\
\hline actc1 & 9 & 23 & 24 & 88 & 17 & 8 & 22 & 20 & 19 & 10 & 6 & 76 & 9 & 17 & 9 & 69 \\
\hline g6pd & 525 & 489 & 478 & 233 & 509 & 373 & 804 & 115 & 425 & 366 & 265 & 128 & 599 & 566 & 724 & 158 \\
\hline tubb2b (N-tubulin) & 15 & 29 & 24 & 9.367 & 20 & 20 & 70 & 1.787 & 12 & 7 & 17 & 30 & 12 & 14 & 46 & 34 \\
\hline vGlut1 & 1 & 1 & 1 & 13 & 1 & 1 & 1 & 3 & 1 & 2 & 1 & 1 & 1 & 1 & 3 & 1 \\
\hline NEG_A & 4 & 8 & 5 & 2 & 2 & 6 & 5 & 5 & 6 & 9 & 3 & 1 & 5 & 7 & 9 & 2 \\
\hline NEG_B & 5 & 7 & 6 & 1 & 5 & 3 & 2 & 1 & 3 & 2 & 1 & 2 & 3 & 5 & 2 & 5 \\
\hline NEG_C & 1 & 1 & 1 & 1 & 1 & 1 & 4 & 2 & 3 & 1 & 1 & 1 & 5 & 1 & 4 & 2 \\
\hline NEG_D & 9 & 7 & 5 & 3 & 6 & 5 & 7 & 6 & 3 & 4 & 5 & 5 & 6 & 3 & 4 & 4 \\
\hline NEG_E & 6 & 10 & 5 & 4 & 4 & 1 & 13 & 8 & 5 & 9 & 6 & 3 & 5 & 4 & 6 & 3 \\
\hline NEG_F & 4 & 6 & 11 & 9 & 2 & 7 & 6 & 8 & 9 & 6 & 4 & 4 & 7 & 7 & 7 & 2 \\
\hline NEG_G & 3 & 1 & 3 & 1 & 1 & 1 & 2 & 3 & 1 & 1 & 1 & 1 & 1 & 1 & 2 & 1 \\
\hline NEG_H & 1 & 3 & 3 & 2 & 4 & 1 & 4 & 1 & 4 & 1 & 1 & 1 & 1 & 1 & 1 & 1 \\
\hline POS_A & 6.711 & 9.436 & 8.775 & 8.486 & 6.152 & 5.987 & 7.380 & 8.693 & 8.133 & 5.911 & 6.992 & 6.544 & 6.507 & 7.327 & 7.343 & 8.616 \\
\hline POS_B & 3.569 & 4.606 & 4.440 & 4.494 & 3.352 & 3.048 & 3.757 & 4.104 & 4.199 & 3.001 & 3.329 & 3.295 & 3.355 & 3.465 & 3.676 & 4.352 \\
\hline POS_C & 1.089 & 1.503 & 1.277 & 1.334 & 1.031 & 933 & 1.165 & 1.237 & 1.321 & 935 & 970 & 998 & 999 & 1.028 & 1.156 & 1.304 \\
\hline POS_D & 221 & 292 & 272 & 312 & 222 & 194 & 245 & 280 & 293 & 201 & 226 & 206 & 221 & 237 & 252 & 251 \\
\hline POS_E & 33 & 61 & 51 & 41 & 32 & 34 & 43 & 42 & 44 & 36 & 35 & 43 & 41 & 46 & 32 & 51 \\
\hline POS_F & 28 & 43 & 60 & 38 & 46 & 43 & 59 & 41 & 50 & 29 & 32 & 18 & 26 & 43 & 29 & 33 \\
\hline
\end{tabular}




\subsection{Summary of the genes analysed with the Nanostring}

Table 6.17 Summary of the genes analysed with the Nanostring. Given is the symbol of the gene and its accession number as well as the target region and the target sequence of the reporter probe. (Grey marks a non-functional H4 probe).

\begin{tabular}{|c|c|c|c|}
\hline Gene & Accession & $\begin{array}{l}\text { Target } \\
\text { Region }\end{array}$ & Target Sequence \\
\hline Sox1 & $\begin{array}{l}\text { NM_001095 } \\
674.1\end{array}$ & $1663-1763$ & $\begin{array}{l}\text { CTGTGCTCATCCTTTTAGAGACAATGCGTCCAGCCTGCTCATCAAGGATCGGGCAGCACACTTGCCTCCACTTTG } \\
\text { CCACAACTGCCCTCTCTTTTTCTTT }\end{array}$ \\
\hline Sox2 & $\begin{array}{l}\text { NM_001088 } \\
222.1\end{array}$ & $901-1001$ & $\begin{array}{l}\text { CCGGGCATGTCTCTGGGATCCATGGGCTCGGTAGTCAAGTCGGAATCCAGCTCCAGTCCACCTGTAGTCACCTC } \\
\text { TTCTTCCCATTCGCGGGCTCCGTGCC }\end{array}$ \\
\hline Sox3 & $\begin{array}{l}\text { NM_001090 } \\
679.1\end{array}$ & $998-1098$ & $\begin{array}{l}\text { GCTCACTCACATATAACACTTTGTGCCCTTTGCTAAAGACGCTTTACTTGCCTGCTGGCAACTATCAGACTGCCGC } \\
\text { ATAAAACATTTAAAAAAAAAAATC }\end{array}$ \\
\hline SoxD & NM_001087 & $684-784$ & $\begin{array}{l}\text { CCCTGGAAGTGGAGGGAACCTCTTTCACGGACCTCTGACCCCTGTGACTGAATGGGACATTCCGAGGGTCTCCG } \\
\text { CTCTACTGACTTGAGAATCTAGGGGA }\end{array}$ \\
\hline $\mathrm{NCAMa} / \mathrm{b}$ & $\begin{array}{l}\text { NM_001087 } \\
827.1\end{array}$ & $44-144$ & $\begin{array}{l}\text { CATCTGGACTTTATATTTCATAGGAACTGCAGTGGCGTTGGAAGTGAACATTGTTCCAGATCAAGGAGAAATAAG } \\
\text { CCTTGGGGAGTCCAAATTCTTCCTG }\end{array}$ \\
\hline Ngn1 & DC113851.1 & $1121-221$ & $\begin{array}{l}\text { CCGACTATGACACCTGCAGCCAGATGTCCTATTCCTCTTCAGATGATGAGGACTCCTTCAGCATGCAGTCTTCTT } \\
\text { CTCCCTTCTCCTCTGGACACATGAC }\end{array}$ \\
\hline $\begin{array}{l}\text { Ngnr-1a } \\
(\mathrm{Ngn} 2 \mathrm{a})\end{array}$ & $\begin{array}{l}\text { NM_001088 } \\
333.1\end{array}$ & $274-374$ & $\begin{array}{l}\text { TGGGTTATTATAGTAAGTGGATGTGAAGTTGTCAGTGCAACATTGGGGCTAACCATTGGCTGTGTGTTTGCGCTT } \\
\text { GTCTAGGATGGTGCTGCTCAAGTGC }\end{array}$ \\
\hline $\begin{array}{l}\text { Nrgn-1b } \\
(\text { Ngn2-b) }\end{array}$ & $\begin{array}{l}\text { NM_001088 } \\
335.1\end{array}$ & $1172-1272$ & $\begin{array}{l}\text { CATCGTTAGCTATGTGTATTAGGAAACTGTCTATCCCTCATCTGCACCTGTTAGACTACAGCTACCAACTTCCTGT } \\
\text { 2TACCAGGGGGCTACTGGGTAATGT }\end{array}$ \\
\hline Ngn3 3 & $\begin{array}{l}\text { NM_001134 } \\
785.1\end{array}$ & $216-316$ & $\begin{array}{l}\text { AAAGAAGCAAAGGGTCAAGCGAATGAGATCAAAAGTAAAGAGCGACGGCGTCGTCGTAAAACAGAGGAGAAATC } \\
\text { GCAGGGTGAAAGCCAATGACAGAGAG }\end{array}$ \\
\hline Ascl1/Xash1 & $\begin{array}{l}\text { NM_001085 } \\
778.1\end{array}$ & $142-242$ & $\begin{array}{l}\text { CTCCTCCTGCCCAGCAGCCTGTCAGCATGGACAACTGCGTCGCTGCCAAGATCATGGACAGCAACTTATCCAGC } \\
\text { CAGCAGCAGCATTTCCTACAGCCCCA }\end{array}$ \\
\hline Mxi1 & $\begin{array}{l}\text { NM_001095 } \\
701.1\end{array}$ & $2418-2518$ & $\begin{array}{l}\text { GCTGCTGCAGGTCATTATCATTCTGCAATCTGTGTTTGGGTGGACTTCCCCTTTAATCTGTTACAAACCAGAAATG } \\
\text { ACACATCCCAGAATCCTCAGCTCC }\end{array}$ \\
\hline NeuroD1a/b & $\begin{array}{l}\text { NM_001085 } \\
794.1\end{array}$ & $370-470$ & $\begin{array}{l}\text { GCCCTGGACAGTCTGCGCAAAGTTGTGCCCTGCTACTCCAAAACACAAAAGTTGTCTAAGATTGAAACTCTGCGC } \\
\text { CTGGCTAAGAACTACATCTGGGCTC }\end{array}$ \\
\hline $\begin{array}{l}\text { NeuroD4 } \\
\text { (Xath3) }\end{array}$ & $\begin{array}{l}\text { NM_001087 } \\
744.1\end{array}$ & $665-765$ & $\begin{array}{l}\text { AGTAATGGATCCTTCTGTGGTAACCCATACACTTAACTGTACCACTCCACCATATGAAGGAGCTCTAACACCTCCA } \\
\text { CTCAGCATCGGTGGTAATTTTTCT }\end{array}$ \\
\hline $\begin{array}{l}\text { NeuroD6 } \\
\text { (Xath2) }\end{array}$ & $\begin{array}{l}\text { NM_001085 } \\
749.1\end{array}$ & $732-832$ & $\begin{array}{l}\text { TACAAGATCCCCATATACTTCTGTTTACCCACCTTATCACAGTCCTGAGCTTAGTACCCCTCCAAGTCACGGGACC } \\
\text { CTTGATAATTCCAAAACCATGAAA }\end{array}$ \\
\hline MyT1 & $\begin{array}{l}\text { NM_001088 } \\
192.1\end{array}$ & $684-784$ & $\begin{array}{l}\text { GAGCTAAACAATGAAAAGCCAACTTCAGTAAAGTCGGGTCAAGCGGAAATAGAACAACTTATGGTAGAAGAGGCG } \\
\text { TGTGAGAAAGAAATCATCATCCAGA }\end{array}$ \\
\hline Ebf2-a/b & $\begin{array}{l}\text { NM_001085 } \\
677.1\end{array}$ & $1085-1185$ & $\begin{array}{l}\text { CATACAAATCCAAACAGTTCTGCAAAGGAGCCCCAGGGAGGTTTATTTACACAGCTTTAAATGAGCCTACAATTGA } \\
5 \text { TTATGGATTCCAGAGGCTGCAAAA }\end{array}$ \\
\hline Ebf3 (Coe3) & $\begin{array}{l}\text { NM_001090 } \\
332.1\end{array}$ & $1998-2098$ & $\begin{array}{l}\text { ATGGGAGTAAACTCTTTCAGCAGCCAGCTAGCAGTTAATGTTTCAGAGACCTCTCAAGCTAATGACCAAGTAGGT } \\
\text { 3 TACAGTCGCAACACAAGCAGTGTTT }\end{array}$ \\
\hline $\begin{array}{l}\text { RBM38/Seb4 } \\
\text { R }\end{array}$ & $\begin{array}{l}\text { NM_001089 } \\
144.1\end{array}$ & $1053-1153$ & $\begin{array}{l}\text { GTAAGAAAATGCTGCAGTCAAGTTCTGTGGATCCCAGCTTTCAGTTCTGTTTCAGGCAATATCAAGGTCATGGATT } \\
\text { 3TTTTGGCGGATTCCAGGCAAGAG }\end{array}$ \\
\hline Pak3 & $\begin{array}{l}\text { NM_001085 } \\
763.1\end{array}$ & $2397-2497$ & $\begin{array}{l}\text { GCATTTGAGTGGAAAGAGGCCCATTTGTTGCTGAACGATCCTGGCACCTTGTGCTTTGCTTTCCTGGCAGTGAAG } \\
\text { AGGTTAGCAGCCTGAATCTATGGCA }\end{array}$ \\
\hline \begin{tabular}{|l|l} 
Cdknx \\
(p27Xic1)
\end{tabular} & $\begin{array}{l}\text { NM_001114 } \\
803.1\end{array}$ & $1076-1176$ & $\begin{array}{l}\text { ITAACCGTGAAACAGCTTGAATCTATTGCTCAGGGATGAACTACCTATGGCTCTCCAGATGTCGTTGAATTACAGC } \\
\text { TCCCAACGGAGTCGTGGCTCAGCA }\end{array}$ \\
\hline Nrp1 & $\begin{array}{l}\text { NM_001087 } \\
911.1\end{array}$ & & $\begin{array}{l}\text { GGAATACTGACGCAACTGATGTAGTCTACCGGCCATTCTCTAAACCTGTTATCACTCGCTTTGTTAGACTCCGTCC } \\
\text { GGTTACATGGGAAAATGGAATATC }\end{array}$ \\
\hline $\begin{array}{l}\text { tubb2b (N- } \\
\text { tubulin) }\end{array}$ & $\begin{array}{l}\text { NM_001086 } \\
064.1\end{array}$ & $900-1000$ & $\begin{array}{l}\text { TGCACTTTTTTATGCCAGGCTTTGCCCCATTAACAAGTCGTGGCAGCCAACAATACCGAGCCCTGACAGTGCCAG } \\
\text { AACTAACACAGCAAATGTTTGATTC }\end{array}$ \\
\hline Pax2-a & $\begin{array}{l}\text { NM_001086 } \\
361.1\end{array}$ & $1494-1594$ & $\begin{array}{l}\text { GAGTGGCCTACTCTGCTTTCCCTACTGACGTTTCTCAGCTGTAATAAGCTGCTCTTACCTCTGCTGCAAGCCTCTT } \\
\text { TGTTGTTATTGTGCACGGTAGCTA }\end{array}$ \\
\hline Twist & $\begin{array}{l}\text { NM_001085 } \\
883.1\end{array}$ & $619-719$ & $\begin{array}{l}\text { AGGGAGCCTGGTCCATGTCTGCATCTCACTAACAGCAATGCCACTACAGCTCAGGCCACACACAAAGATTATACT } \\
\text { TATACCAATGAATGGGAAAAAAAAC }\end{array}$ \\
\hline $\mathrm{Hb} 9 / \mathrm{mnx} 1$ & $\begin{array}{l}\text { NM_001096 } \\
823.1\end{array}$ & $1727-1827$ & $\begin{array}{l}\text { GTTACACAGGCTACTGGGAGTCCCCTACTTTTGTGTTGACACCATCATAAGAAAACCCGCTGGGCACCTGAAGGC } \\
\text { 7ACACTATAGGCTGTCTTTATTTTT }\end{array}$ \\
\hline Hox11L2/TIx3 & $\begin{array}{l}\text { NM_001085 } \\
746.1\end{array}$ & $186-286$ & $\begin{array}{l}\text { TCGTGTCTCTCCGCTAATTTCCCCGCCGGCATCGGGGCCACGTTTGATGAGTCGGGATGTTACAGTGTCAATGT } \\
\text { GAGTCTGGCTCCTGCCGGAGTGATTA }\end{array}$ \\
\hline Hpx6 (Lbx1) & $\begin{array}{l}\text { NM_001095 } \\
723.1\end{array}$ & $30-130$ & $\begin{array}{l}\text { GCGTCCTCGGTGGAGGAAAGAAGAAGGAATGCCTTGGACCTTTTACCACCTCCTGCTAATTCTAACAAGCCCCTG } \\
\text { ACTCCTTTCAGCATTGAGGATATCC }\end{array}$ \\
\hline Lhx1 (Lim1) & $\begin{array}{l}\text { NM_001090 } \\
659.1\end{array}$ & $21-121$ & $\begin{array}{l}\text { TCTATTCTCCTAATCCGCCCATTCCTCTAAAATCCCAAATAACCAAAGGCAATGGTTCACTGTGCTGGATGCGAAA } \\
\text { GGCCCATTCTGGACCGTTTCTTGT }\end{array}$ \\
\hline Lhx2 & AJ311712.1 & $264-364$ & $\begin{array}{l}\text { GTATACTGCCGGCTCCACTTTGAGACTTTGATACAGGGAGAATACCAAGTTCATTTCGGTCACTCGGATGTAGCA } \\
\text { TCAAGGAAGGGCTCAGGGGCTACCT }\end{array}$ \\
\hline Lhx5 & $\begin{array}{l}\text { NM_001090 } \\
569.1\end{array}$ & $440-540$ & $\begin{array}{l}\text { CCAGGGACATTTGCGTACAAGGCCCGCTGGGACTCAAGTTATATTTGGGTCTTTGGTTGGGGAGTCTATGAGGA } \\
\text { TTTCACTGGACTTGGCTTCTGCTGCA }\end{array}$ \\
\hline$L m \times 1 b$ & $\begin{array}{l}\text { NM_001090 } \\
433.1\end{array}$ & $688-788$ & $\begin{array}{l}\text { AGGACAATCCTCACAACCCAACAAAGAAGGGCGTTCAAAGCATCTTTTGAGGTTTCATCGAAACCCTGTAGGAAG } \\
\text { GTCAGAGAGACATTAGCTGCTGAAA }\end{array}$ \\
\hline runx1 (Xaml) & $\begin{array}{l}\text { NM_001086 } \\
497.1\end{array}$ & $1150-1250$ & $\begin{array}{l}\text { CTGACAGCGTTCAGTGATCCTCGAGTTGGCATTGACCGACAGTTCTCCACTCTTCCTTCCATCTCTGATCCACGT } \\
\text { ATGCACTACCCAGGGGCTTTTACCT }\end{array}$ \\
\hline Olig3 & AF035446.1 & $1112-212$ & $\begin{array}{l}\text { ACTGTCTCTTGAGACCCCTGAGCATAACTATTGACTCCTGGGAGCGATCACTGACTGCCAGCTGTTGCGACTTGC } \\
\text { CCATTTAAAAGTGCATTTACTCACC }\end{array}$ \\
\hline $\begin{array}{l}\text { FoxD3a } \\
\text { (XfD6) }\end{array}$ & $\begin{array}{l}\text { NM_001085 } \\
557.1\end{array}$ & $91-191$ & $\begin{array}{l}\text { GCAGCAAGTTCACTACTACTTGCCCTACCGAGCCTCTCTCTATTTCTCTTCTCTGCCCATCGCTGTGGAGCGTAA } \\
\text { CTGGAATGACCCTGTCAGGCAGCGG }\end{array}$ \\
\hline vGlut1 & $\begin{array}{l}\text { NM_001089 } \\
635.1\end{array}$ & $2177-2277$ & $\begin{array}{l}\text { AGTGAATTGAGTTAGATGTACACATTACTGCGTGATTCCCAGGGATACGGTGTAGAAAATATCCATGAGGATGGG } \\
\text { GCATGATCAATTCTACATCACTTGG }\end{array}$ \\
\hline Gad1 & $\begin{array}{l}\text { NM_001085 } \\
801.1\end{array}$ & $794-894$ & $\begin{array}{l}\text { GGCAGCAGTCCCCAGATTGGTCCTGTTTACTTCAGAACACAGCCACTATTCCATAAAGAAAACTGGCGCGGCATT } \\
\text { AGGATTTGGAAGTGAAAATGTGATC }\end{array}$ \\
\hline Krox20/egr2 & $\begin{array}{l}\text { NM_001085 } \\
779.1\end{array}$ & $273-373$ & $\begin{array}{l}\text { AGGAGATGGAATGATCAGTGTGGATATGAATAATGACAAGCGGTCGCTGGATTTCTCCTACTCGTCCAACTACCC } \\
\text { CTTGGCCCCTAGGACTCAGCCGATC }\end{array}$ \\
\hline Eng1-a/b & D14693.1 & $122-222$ & $\begin{array}{l}\text { CCCTGTGCACAAGAAAGACTCTGTGGTGTGGCCCGCCTGGGTCTATTGCACTCGCTACTCCGACCGACCCTCCT } \\
\text { CTGGCCCTAGGACCCGCAAGCTAAAA }\end{array}$ \\
\hline Islet1 (isl1) & NM_001110 & $1102-1202$ & $\begin{array}{l}\text { TACAGAGTGACATTGATCAGCCGGCCTTCCAGCAACTGACCTGTTTCTCACAGGTTAATTTTTCAGAAGGAGGAC } \\
\text { CTGGCTCTAACTCTACAGGGAGTGA }\end{array}$ \\
\hline Insm1 & $\begin{array}{l}\text { NM_001110 } \\
719.1\end{array}$ & $2177-2277$ & $\begin{array}{l}\text { CAAATGACTGTGTCCGCCCATGAGTCGCTTCTACAATGAGATAAATGTATATTGCCCAGTTGCCATCCACTCACTG } \\
\text { TTACAGCATTTGTGATGCCCTGAC }\end{array}$ \\
\hline Nkx6.1 & $\begin{array}{l}\text { NM_001099 } \\
916.1\end{array}$ & $172-272$ & $\begin{array}{l}\text { AATACATCATTCCCTCTGCAGCTTGAACTGATTTAAACCTCGGCCATCCAACACTACAGCCCAGCGTCGGTAGGA } \\
\text { TATAGTGATTCACCCTTGGAGTTGT }\end{array}$ \\
\hline Nkx6.2 & $\begin{array}{l}\text { NM_001096 } \\
886.1\end{array}$ & $28-128$ & $\begin{array}{l}\text { TGTTTGTCGCCGCTGCATGTGTGGGATCATTACTGCCCTGAAACTTGCGCTGATCGCCGGGAAGTTGCGACAGA } \\
\text { AGCCGTTTGCCTCGGGGACTTCTCTC }\end{array}$ \\
\hline Pax3 & $\begin{array}{l}\text { NM_001095 } \\
524.1\end{array}$ & $532-632$ & $\begin{array}{l}\text { AAAAGAGACAACCCGGGTATGTTCAGCTGGGAAATTCGCGATAAATTACTTAAAGACGGAGTTTGTGATCGCAAT } \\
\text { ACTGTGCCTTCAGTAAGCTCCATCA }\end{array}$ \\
\hline Pax6-a & NM_001085 & $1551-1651$ & 1 CTGCCCACAAGTCCATCTGTGAATGGGCGGAGCTATGACACATACACACCTCCCCACATGCAGACACATATGAAC \\
\hline
\end{tabular}




\begin{tabular}{|c|c|c|c|}
\hline & 944.1 & & \\
\hline Pax6-b & $\begin{array}{l}\text { NM_001172 } \\
195.1\end{array}$ & $1339-1439$ & $\begin{array}{l}\text { CTTACAGTGCTTTGCCACCTATGCCTAGTTTTACGATGGGCAACAATCTACCTATGCAAGTCTCATTTCCCCTGGA } \\
\text { GTGTCAGTCCCAGTTCAAGTACCC }\end{array}$ \\
\hline c-myc & $\begin{array}{l}\text { NM_001085 } \\
896.1\end{array}$ & $523-623$ & $\begin{array}{l}\text { TCTGTCTTCTTCTTCTCCGTGTCCGAGTCAACCACCACCGAGCCCGCTTAAGTCTCCCTCGTGTCATGGGAGCCT } \\
\text { GAGTCTGGGAGGGACCCACAGGAGC }\end{array}$ \\
\hline Slug & $\begin{array}{l}\text { NM_001086 } \\
880.1\end{array}$ & $87-187$ & $\begin{array}{l}\text { GGACACTCACTGAGCAAGGACTGGGCGCATTCACTCCACTGTAATTACGGCAAGTGACAAGGGAAGAGGCTGCG } \\
\text { AGTATAACACTGCGGGGCCGCTCACT }\end{array}$ \\
\hline RBPJ & $\begin{array}{l}\text { NM_001090 } \\
878.1\end{array}$ & $1537-1637$ & $\begin{array}{l}\text { ACATTTCCGCATTTCGAGAAGGTTGGAGATGGGTTCGTCAGCCAGTGCAAGTGCCTGTTACGTTGGTCCGAAAC } \\
\text { GACGGTGTAATCTATTCAACCAGCCT }\end{array}$ \\
\hline Notch1 & $\begin{array}{l}\text { NM_001087 } \\
605.1\end{array}$ & $468-568$ & $\begin{array}{l}\text { ACTGGGGTGTGCCTATGCGGTAATTTGTATTTCGGTGAAAGGTGCCAGTTCCCCAATCCCTGCACCATAAAGAAT } \\
\text { CAGTGTATGAACTTTGGAACCTGCG }\end{array}$ \\
\hline DII1 (Delta1) & BC070634.1 & $628-728$ & $\begin{array}{l}\text { CTGAAGTACTCCTACCGATTTGTGTGTGATGAATACTACTATGGGGAGGGCTGCTCTGACTACTGCCGCCCCAGA } \\
\text { GATGATGCTTTCGGCCATTTTTCCT }\end{array}$ \\
\hline Delta2 & U70843.1 & $1537-1637$ & $\begin{array}{l}\text { TCTAACTGTGAAAAGAAGATTGATCGCTGTACCAACAGTCCCTGTCTGAATGGAGGTCAATGTCTAGATATGGGC } \\
\text { CGAAATGTTTTGTGCAAGTGTCGCC }\end{array}$ \\
\hline $\begin{array}{l}\text { Jagged1 } \\
\text { (Serrate-1) }\end{array}$ & $\begin{array}{l}\text { NM_001090 } \\
307.1\end{array}$ & $136-236$ & $\begin{array}{l}\text { GTGCATTCAGGATCGGCGTGCCCTACAACAAGAGCCTTGAAAAGTGTGTGTTGTGTTGCAGTCGTGCATGCTCAA } \\
\text { ATCGCCTGAGTATATCAGAGGTGCG }\end{array}$ \\
\hline $\begin{array}{l}\text { Hes5.1 } \\
\text { (ESR1) }\end{array}$ & $\begin{array}{l}\text { NM_001095 } \\
627.1\end{array}$ & $326-426$ & $\begin{array}{l}\text { CAGAAAATGGAGAAACCCAGATGAAACTGCTTAATCACCTGCAAGCACCCCAAAGGCTCTCAGTTGCCCCTCACA } \\
\text { CCTATATCCCCAGTGTTTCTGACTC }\end{array}$ \\
\hline $\begin{array}{l}\text { ESR6e } \\
\text { (Hes3.1) }\end{array}$ & $\begin{array}{l}\text { NM_001088 } \\
503.1\end{array}$ & $267-367$ & $\begin{array}{l}\text { GCTTTCTGCATACCCAGGAGCACGGACACATTCAAGAAGGAAATAAAAATCTACCGTGGACTGATTCTTGCCTCC } \\
\text { CTGCACCCTCTCAAAAATTCTACCA }\end{array}$ \\
\hline ESR9.1a & $\begin{array}{l}\text { NM_001088 } \\
237.1\end{array}$ & $556-656$ & $\begin{array}{l}\text { GATATCCTGCAGACTGGTGGCTTACCTGATGGTTTATTATGACTATGTAATAGAATAACTGGTTACTGTAATGGTG } \\
\text { CAACACCAAAGCTATTTTGTAGCT }\end{array}$ \\
\hline ESR10 & AJ009285.1 & 824-924 & $\begin{array}{l}\text { TTGCTTGGGTATAAATGTATTATGGGCTCACATAGGGGAGTCAGAAGTACATGCTGTGTATTGCCACACATTGCG } \\
\text { GCATATTTGGGCCTCTCAGAGTCTG }\end{array}$ \\
\hline Hes6.1 & BC130161.1 & $1166-1266$ & $\begin{array}{l}\text { TCCTGGCTTTCAGCCATCTAAAGATTGTTGGGATACAACTCTCGCTAACTAGAATGACAAGTTACACTTCAGTAAC } \\
\text { AGTTTGAGGGCCATGTATCTCTCC }\end{array}$ \\
\hline Hes2 & $\begin{array}{l}\text { NM_001122 } \\
882.1\end{array}$ & $799-899$ & $\begin{array}{l}\text { AGTGACCCACATTGTGAACCCAAAGGGTTTAATCTAGTATGCGATTTGCATTATTTTACATTAACCCATGGACTGC } \\
\text { CAGAGATGGCCAATGCTACAGTCT }\end{array}$ \\
\hline Hes1a(Hairy1) & $\begin{array}{l}\text { NM_001087 } \\
927.1\end{array}$ & $557-657$ & $\begin{array}{l}\text { GAAGGGGTCAACACAGATGTCCGGACCCGACTCCTGGGGCATCTTGCCAACTGCATGAACCAGATCAATGGCAT } \\
\text { GAACTACCCTACCCAGCCCCAGATGC }\end{array}$ \\
\hline Hes7 (XHR1) & $\begin{array}{l}\text { NM_001088 } \\
706.1\end{array}$ & $490-590$ & $\begin{array}{l}\text { CGCATTTAGTGTCCAACAGCATCTCCATCAGCCCTACAAAGACTCTGGTTGACAGTCATTTCACTTACCAAACCTT } \\
\text { CAAGACCTGGAGACCCTGGGTATG }\end{array}$ \\
\hline Xbra-a/b & $\begin{array}{l}\text { NM_001090 } \\
578.1\end{array}$ & $534-634$ & $\begin{array}{l}\text { AGCCCACTGGATGAAAGATCCTGTCTCTTTTAGCAAAGTCAAACTTACAAACAAAATGAATGGTGGAGGCCAGAT } \\
\text { TATGTTAAACTCTTTGCACAAGTAT }\end{array}$ \\
\hline Arx-a & $\begin{array}{l}\text { NM_001085 } \\
860.1\end{array}$ & $246-346$ & $\begin{array}{l}\text { GCGCCCACAGCATTCCTTCCCTTGCACATCGGCTAGATCCGGACAAAACTTTGGAAGGGTCCCCTAAGGGCAAT } \\
\text { GCTCCATTTGAAGGGGATTTGCACCT }\end{array}$ \\
\hline PRDM14 & & $650-750$ & $\begin{array}{l}\text { CTGACAGTGCTGGACACTTCCTATGGGACTTTGCCCAAATCTGGAGTTTTCTGCAAGAACCTCATCCCTAAAGGA } \\
\text { GCCAAGTTCGGTCCATTTCAAGGCA }\end{array}$ \\
\hline Ptf1a-a/b & AY372268.3 & 149-249 & $\begin{array}{l}\text { CATTCCTCTAGGGACGCCCTGGACGCAGACGACTTTTTGGAAGACGATGTAGACTTCTTGGCCGGTCAGATCCA } \\
\text { AGACTATTACAGAGACAGCCGAGTGC }\end{array}$ \\
\hline FGF8 & $\begin{array}{l}\text { NM_001090 } \\
435.1\end{array}$ & $47-147$ & $\begin{array}{l}\text { CTCCGAGCCTCTCAGTCAGCTTCTAGTGTTATTGGCCGGTGGGGTCGGCGCTGCTGGTGCAGACAAGAAGCAAG } \\
\text { TCTTACTCATCAACACTTTCATCATC }\end{array}$ \\
\hline Numb & BE189039.2 & $360-460$ & $\begin{array}{l}\text { ACCATGCAATACTGCGCGTCCATTTTCGGTAAAAGTAGAACAGAGACATTTCTCGTGGTTGTGATTGTTTAAAGCA } \\
\text { ATGTGCTACATACAAGGAAGGTGG }\end{array}$ \\
\hline Numbl & GQ214766.1 & $625-725$ & $\begin{array}{l}\text { AGGCGATGGATCTGTCACTGCTTCATGACTGTTAAAGATACGGGAGAGAGGCTCAGCCATGCTGTTGGATGTGC } \\
\text { ATTTGCCGCTTGTCTGGAGAGGAAGC }\end{array}$ \\
\hline actc1 & $\begin{array}{l}\text { NM_001086 } \\
591.1\end{array}$ & $1126-1226$ & $\begin{array}{l}\text { CTCCATTGTGCACAGGAAGTGCTTCTAAACATTACACACACTCCTCCAAGCAACATATACAGAGTACCTCAAAGTC } \\
\text { AAAGAAAACAAGACCTGGACAGTC }\end{array}$ \\
\hline ODC & $\begin{array}{l}\text { NM_001086 } \\
698.1\end{array}$ & $855-955$ & $\begin{array}{l}\text { GGATATAATTGGTGTGAGTTTCCATGTTGGCAGTGGCTGCACTGATCCACAGACTTATGTACAAGCTGTCTCAGA } \\
\text { TGCACGATGTGTCTTTGACATGGGG }\end{array}$ \\
\hline $\mathrm{H} 4$ & $\begin{array}{l}\text { NM_001094 } \\
457.1\end{array}$ & $655-755$ & $\begin{array}{l}\text { CCACGCCCCTTCTCCCCATAAAATCAGTTACAGGCTCTCGGGCTCTTTTGCTCTTGCCGGATGGAAATTACTGTT } \\
\text { GCTGCTCAGCGTCTCACAGAACTCC }\end{array}$ \\
\hline gapdh & \begin{tabular}{|l|l|l} 
NM_001087 \\
098.1
\end{tabular} & $773-873$ & $\begin{array}{l}\text { ACCTGCCGCCTGCAGAAGCCGGCCAAGTACGATGACATCAAGGCCGCCATTAAGACTGCATCAGAGGGCCCAAT } \\
\text { GAAGGGAATCCTGGGATACACACAAG }\end{array}$ \\
\hline actb & $\begin{array}{l}\text { NM_001088 } \\
953.1\end{array}$ & $1179-1279$ & $\begin{array}{l}\text { ATGCTTCTAAAGGACAGACCCTTTCAACATGAACAAATGTACCTGTGCAGGAAGATCACATTGGCATGGCTTTACT } \\
\text { CTTTTGTTGGCGCTTGGCTCAGAA }\end{array}$ \\
\hline g6pd & $\begin{array}{l}\text { NM_001086 } \\
550.1\end{array}$ & $862-962$ & $\begin{array}{l}\text { GTGGAGGATACTTTGACGAATTTGGCATCATCCGGGATGTCATGCAAAATCACTTGCTCCAAATGATGTGTTTGAT } \\
\text { GGCTATGGAGAAGCCGGTCTCCAC }\end{array}$ \\
\hline HNF6 gene 2 & DC109227.1 & $141-241$ & $\begin{array}{l}\text { TGTGTCCCAGTTGGCAATGTTATTGGAAGCTTTACTCTTATGAGAGAGGACAGAGGTTTGGGGCCACCAAGTAAC } \\
\text { TTTTACAGTCACTATCCCAAAGACA }\end{array}$ \\
\hline Pdia2 (XPDip) & $\begin{array}{l}\text { NM_001090 } \\
179.1\end{array}$ & $881-981$ & $\begin{array}{l}\text { TGCTGCCCAGATCCCAAACCACTTGTTGCTGTTTATCAATAAGAGTGACGATTCCCAACTGGTGCTGCTGGAACA } \\
\text { TTTCCGCAAAGCAGCTCCTGACTTT }\end{array}$ \\
\hline Sox9-a/b & $\begin{array}{l}\text { NM_001090 } \\
807.1\end{array}$ & $441-541$ & $\begin{array}{l}\text { GAAGTTCCCCGTGTGCATCAGAGAAGCGGTCAGCCAGGTGTTGAAGGGATATGATTGGACCCTGGTACCGATGC } \\
\text { CAGTCAGAGTTAATGGATCCAGCAAG }\end{array}$ \\
\hline $\mathrm{Hmx3}$ & $\begin{array}{l}\text { NM_001085 } \\
757.1\end{array}$ & $356-456$ & $\begin{array}{l}\text { TTAAAGGGGCCCTGGACGGAGCAGCGTTTGCCTTCTCCCCGCTGACAGATTTCACCTTCCCTCGGCTAGAGATC } \\
\text { CCGACTCAAAGGTTCGCCCTCCCGGC }\end{array}$ \\
\hline Barhl2 & $\begin{array}{l}\text { NM_001088 } \\
552.1\end{array}$ & $1164-1264$ & $\begin{array}{l}\text { CCCGGTGAAACACTATCTATTTACCGAAGCAGCACAGAGTCACCATTGCAGTAATCCAAAAAAAAGGACATCAGA } \\
\text { ACCGGAGACAGATGAGCTTAGTGAT }\end{array}$ \\
\hline Mecom & $\begin{array}{l}\text { NM_001095 } \\
670.1\end{array}$ & $3934-4034$ & $\begin{array}{l}\text { TATTGTGAATCTTGCAATGTCCAGCGCTCAACAAGAGGTGAAGTCATTAAAGGGCCATTTGAATCACTGATACCAC } \\
\text { TAATATGTCTTACAGTTCAATGTT }\end{array}$ \\
\hline Kirrel2 & $\begin{array}{l}\text { NM_001086 } \\
488.1\end{array}$ & $690-790$ & $\begin{array}{l}\text { CCCAGTCGCCAATGTCCTGCTTGATGTACCCTACAATCTCACCTGTCTTGCTTCCGTCGCTAAGCCTGCTGCAGA } \\
\text { GATTACCTGGTTCCGTGATGGAAAG }\end{array}$ \\
\hline Cpa1 & $\begin{array}{l}\text { NM_001095 } \\
030.1\end{array}$ & $517-617$ & $\begin{array}{l}\text { CCCAACCGTCCAGCTTTTTGGATTGACACTGGTATCCATTCCCGTGAGTGGGTCACTCAGGCCAGCGGTATCTG } \\
\text { GTTTGCCAAGAAGATTGCAACAGATT }\end{array}$ \\
\hline PRDM13 & BJ064589.1 & $452-552$ & $\begin{array}{l}\text { TCAGGTCTGCTTTTAAACCAGCTGGACTATCCAAATTACCCAATACTGATTCAGTCAGAGAGGACTCAAAGGCCA } \\
\text { CCAGTATGGGATTCAGCAAACTTTT }\end{array}$ \\
\hline $\begin{array}{l}\text { Ptf1a-a/b } \\
\text { 5'UTR }\end{array}$ & $\begin{array}{l}\text { NM_001174 } \\
\text { O20.1 }\end{array}$ & $4-104$ & $\begin{array}{l}\text { CGAGGAACCGCGGGACCCACTCAGCGCAGTCACCAGGGGGCAGACACCATGGAAACGGTCCTGGAGCAGTTG } \\
\text { GCGGGGCTCGAGTCCTTCCCTTCCCCGT }\end{array}$ \\
\hline $\begin{array}{l}\text { Ascl1/Xash1 } \\
\text { new }\end{array}$ & $\begin{array}{l}\text { NM_001085 } \\
778.1\end{array}$ & $630-730$ & $\begin{array}{l}\text { ACCATCTCCCCCAACTATTCCCACGATATGAACTCTATGGCGGGCTCCCCCGTCTCCTCGTACTCCTCAGATGAA } \\
\text { GGCTCCTATGATCCGCTGAGCCCGG }\end{array}$ \\
\hline RBPJ 3'UTR & $\begin{array}{l}\text { NM_001090 } \\
878.1\end{array}$ & $2034-2134$ & $\begin{array}{l}\text { TTACAAAAATGCGCAAAAAATAGAAAATGTGGCTTGGGCTGAAACCTTCAGCAAGCTAGAGAAGCATGAAGATAA } \\
\text { CATAGTTTTTACGGACCAAGGAACT }\end{array}$ \\
\hline H4 new & $\begin{array}{l}\text { NM_001094 } \\
457.1\end{array}$ & $129-229$ & $\begin{array}{l}\text { GGAGAGGGGGAGTCAAGCGCATCTCTGGCCTCATCTATGAGGAGACTCGTGGGGTCCTCAAGGTTTTCCTGGA } \\
\text { GAATGTCATCCGGGACGCCGTCACCTA }\end{array}$ \\
\hline
\end{tabular}




\subsection{Comparison of genes identified by RNA-sequencing with related publications}

Table 6.18 Comparison of direct Ptf1a and Ptf1a/Su(H) target genes with Ptf1a target genes identified in the adult mouse pancreas by ChIP-sequencing (Masui et al., 2010)

\begin{tabular}{|l|r|r|}
\hline symbol & Ptf1a-GR vs Cc & Ptf1a-GR + SuH-GR vs CC \\
\hline aldh1b1 & 703,6 & 330,9 \\
\hline rpl37 & 2,9 & - \\
\hline rps19 & 2,4 & - \\
\hline slc26a2 & - & 37,9 \\
\hline agrn & - & 5,0 \\
\hline swap70 & - & 3,7 \\
\hline vamp5 & - & 2,9 \\
\hline nfe211 & - & 2,6 \\
\hline zswim4 & - & 2,1 \\
\hline
\end{tabular}

Table 6.19 Comparison of direct Ptf1a and Ptf1a/Su(H) target genes with direct Ptf1a target genes identified in the 2-666 pancreatic progenitor cells by ChIP. sequencing (Thompson et al., 2012)

\begin{tabular}{|c|c|c|}
\hline symbol & Ptf1a-GR vs CC & Ptf1a-GR + SuH-GR vs CC \\
\hline neurod4 & 39,7 & 113,5 \\
\hline nr5a2 & 31,3 & 72,2 \\
\hline gfi1 & 19,2 & 80,9 \\
\hline zc3h12a & 17,4 & 13,9 \\
\hline hes3.3 & 9,2 & 15,9 \\
\hline des.1 & 8,2 & 5,9 \\
\hline cbfa2t2 & 7,0 & 10,0 \\
\hline dll1 & 6,7 & 7,4 \\
\hline $\operatorname{cox} 4 \mathrm{i} 2$ & 6,1 & 5,9 \\
\hline gas6 & 5,5 & 3,9 \\
\hline g0s2 & 5,2 & 2,2 \\
\hline arl4a & 4,5 & 3,4 \\
\hline hes3.1 & 3,5 & 4,5 \\
\hline pou $2 f 3$ & 3,2 & 3,8 \\
\hline cpa1 & - & 65,9 \\
\hline atoh8 & - & 58,5 \\
\hline slc8a2 & - & 25,0 \\
\hline cdh15 & - & 24,0 \\
\hline chad & - & 16,8 \\
\hline pik3r5 & - & 14,6 \\
\hline fat 4 & - & 11,8 \\
\hline fjx1 & - & 10,9 \\
\hline wdr86 & - & 8,3 \\
\hline ampd3 & - & 6,3 \\
\hline Rpl30 & 2,4 & - \\
\hline matn4 & - & 6,0 \\
\hline gpx3 & - & 5,8 \\
\hline $\mathrm{mn} 1$ & - & 5,2 \\
\hline mgat3 & - & 4,8 \\
\hline slit1 & - & 4,8 \\
\hline nrap & - & 4,8 \\
\hline adcy9 & - & 4,5 \\
\hline cldn5 & - & 4,3 \\
\hline vash2 & - & 3,5 \\
\hline kirrel2 & - & 3,5 \\
\hline slc16a12 & - & 2,7 \\
\hline sh3rf1 & - & 2,3 \\
\hline tacc1 & - & 2,3 \\
\hline pitpnm2 & - & 2,2 \\
\hline
\end{tabular}


Table 6.20 Comparison of direct Ptf1a target genes with direct Ngn2 target genes identified in animal caps by Affymetrix Microarray analysis (Seo et al., 2007)

\begin{tabular}{|c|c|c|}
\hline symbol & Ptf1a-GR vs CC & Ptf1a-GR + SuH-GR vs CC \\
\hline dpysl3 & 15,0 & 12,0 \\
\hline hes3.3 & 9,2 & 15,9 \\
\hline $\mathrm{XI.58208}$ & 6,0 & 8,0 \\
\hline tmem56.2 & 4,4 & - \\
\hline rbm24 & 3,4 & 3,7 \\
\hline tiparp & 2,9 & 4,1 \\
\hline XI.18615 & - & 4,6 \\
\hline mafb & - & 3,5 \\
\hline mxra7 & 127,3 & 147,3 \\
\hline neurod4 & 39,7 & 113,5 \\
\hline twist1 & 19,7 & 39,0 \\
\hline znf238 & 11,5 & 20,3 \\
\hline ebf2 & 8,9 & 23,1 \\
\hline cbfa2t2 & 7,0 & 10,0 \\
\hline $\mathrm{XI.76708}$ & 6,7 & 7,8 \\
\hline myt1 & 5,9 & 10,6 \\
\hline amotl2 & 5,5 & 5,2 \\
\hline snai1 & 4,3 & 30,3 \\
\hline plk3 & 3,7 & 6,0 \\
\hline $\mathrm{XI} .71723$ & 3,6 & 3,1 \\
\hline snrk & 3,4 & 2,6 \\
\hline fstl1 & - & 11,2 \\
\hline ascl1 & - & 4,1 \\
\hline zeb2 & - & 2,7 \\
\hline hes6.1 & - & 2,7 \\
\hline
\end{tabular}


Table 6.21 Comparison of direct Ptf1a/Su(H) target genes with identified genes downstream of Ptf1a in endodermal explants (Bilogan and Horb, 2012)

\begin{tabular}{|c|c|c|}
\hline \multirow{5}{*}{ NF32 } & symbol & Ptf1aGR + SuH-GR vs CC \\
\hline & X1.72042 & 4,5 \\
\hline & hes5.1 & 3,7 \\
\hline & crmp1 & 3,4 \\
\hline & des.1 & 2,6 \\
\hline \multirow[t]{7}{*}{ NF36 } & vcan & 3,4 \\
\hline & atp6vod2 & 2,6 \\
\hline & sox9 & 2,4 \\
\hline & unknown & 1,6 \\
\hline & \begin{tabular}{l|l|} 
myh6 \\
\end{tabular} & 1,6 \\
\hline & cebpd & 1,5 \\
\hline & hes4 & 1,0 \\
\hline \multirow[t]{8}{*}{ NF32/NF36 } & cpa1 & 6,0 \\
\hline & $\mathrm{i} 11 \mathrm{~b}$ & 5,2 \\
\hline & thbs3 & 4,6 \\
\hline & $\begin{array}{ll}\text { ebf2 } \\
\end{array}$ & 4,5 \\
\hline & dpysl3 & 3,6 \\
\hline & hey1 & 3,5 \\
\hline & hes5.2 & 2,3 \\
\hline & cldn5 & 2,1 \\
\hline
\end{tabular}

6.5 Primary transcriptome sequencing data

The primary sequencing data are provided on CD "Appendix 6.5" attached to the thesis.

Table 6.22 Candidates_Ngn2-GR_6h_Dex_vs_Cc_6h_Dex

Table 6.23 Candidates_Ngn2-GR_25h_Dex_vs_Cc_25h_Dex

Table 6.24 Candidates_Ptf1a-GR_6h_Dex_vs_Cc_6h_Dex

Table 6.25 Candidates_Ptf1a-GR_25h_Dex_vs_Cc_25h_Dex

Table 6.26 Candidates_Ptf1aW224A_W242A-GR_25h_Dex_vs_Cc_25h_Dex

Table 6.27 Candidates_Ptf1aW224A_W242A-GR_6h_Dex_vs_Cc_6h_Dex

Table 6.28 VennView_Comparison of downstream targets of Ptf1a, Ngn2 and Ptf1aW224A_W242A after 6 and $25 \mathrm{~h}$

Table 6.29 Candidates_Ptf1a_vs_CC_CHX_Dex

Table 6.30 Candidates_SuH_vs_CC_CHX_Dex 
Table 6.31 Candidates_Ptf1a_SuH_vs_CC_CHX_Dex

Table 6.32 VennView_Comparison of identified direct target genes for Ptf1a and $\mathrm{Su}(\mathrm{H})$ alone as well as in combination

Table 6.33 VennView_Comparison of downstream genes of Ptf1a and Ptf1aW224A_W242A after 6 and $25 \mathrm{~h}$ and genes identified as direct targets of Ptf1a alone and with $\mathrm{Su}(\mathrm{H})$ in combination 